\title{
Metering Best Practices, A Guide to Achieving Utility Resource Efficiency, Release 2.0
}

\author{
GP Sullivan WF Sandusky \\ WD Hunt TM Koehler \\ R Pugh BK Boyd
}

August 2011
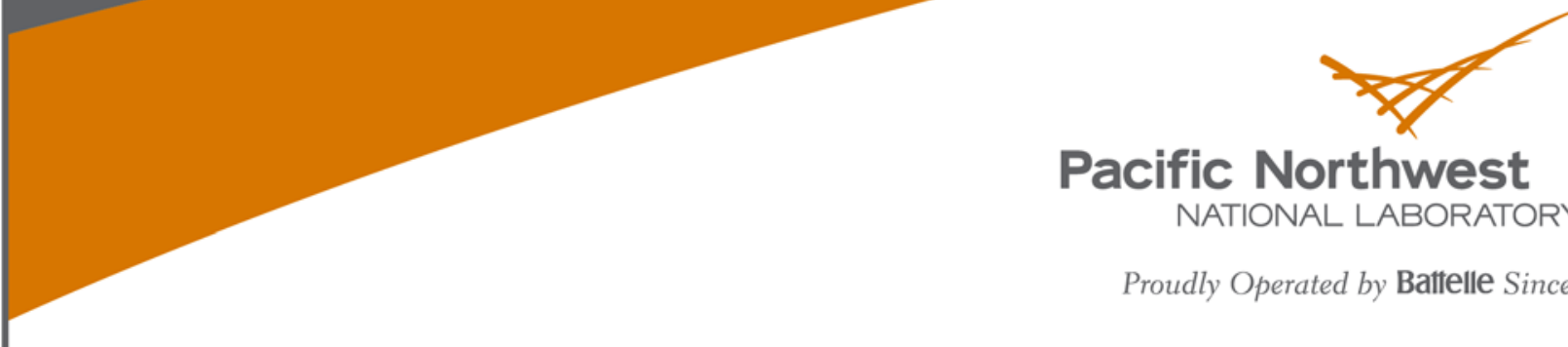

Pacific Northwest

NATIONAL LABORATORY

Proudly Operated by Battelle Since 1965 


\title{
DISCLAIMER
}

This report was prepared as an account of work sponsored by an agency of the United States Government. Neither the United States Government nor any agency thereof, nor Battelle Memorial Institute, nor any of their employees, makes any warranty, express or implied, or assumes any legal liability or responsibility for the accuracy, completeness, or usefulness of any information, apparatus, product, or process disclosed, or represents that its use would not infringe privately owned rights. Reference herein to any specific commercial product, process, or service by trade name, trademark, manufacturer, or otherwise does not necessarily constitute or imply its endorsement, recommendation, or favoring by the United States Government or any agency thereof, or Battelle Memorial Institute. The views and opinions of authors expressed herein do not necessarily state or reflect those of the United States Government or any agency thereof.

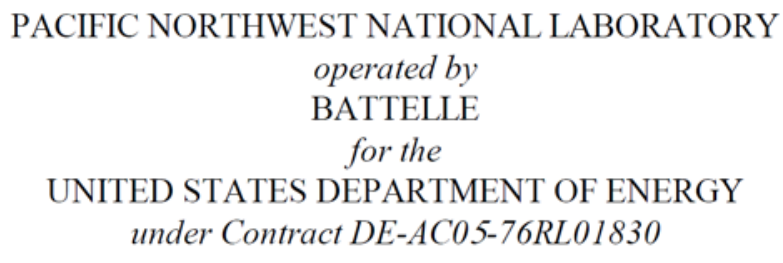

Printed in the United States of America

Available to DOE and DOE contractors from the Office of Scientific and Technical Information,

P.O. Box 62, Oak Ridge, TN 37831-0062; ph: (865) 576-8401 fax: (865) 576-5728

email: reports@adonis.osti.gov

\author{
Available to the public from the National Technical Information Service, \\ U.S. Department of Commerce, 5285 Port Royal Rd., Springfield, VA 22161 \\ ph: (800) 553-6847 \\ fax: (703) 605-6900 \\ email: orders@ntis.fedworld.gov \\ online ordering: http://www.ntis.gov/ordering.htm
}


PNNL-17221

Release 2.0

\title{
Release 2.0
}

\section{Metering Best Practices}

\section{A Guide to Achieving Utility Resource Efficiency}

\author{
GP Sullivan ${ }^{1}$ \\ WD Hunt \\ R Pugh \\ WF Sandusky \\ TM Koehler \\ BK Boyd
}

August 2011

\author{
Prepared for \\ the Federal Energy Management Program \\ U.S. Department of Energy \\ under Contract DE-AC05-76RL01830
}

Pacific Northwest National Laboratory

Richland, Washington 99352

${ }^{1}$ Efficiency Solutions, LLC 
This page intentionally left blank. 


\section{Preface}

This release is an update and expansion of the information provided in Release 1.0 of the Metering Best Practice Guide that was issued in October 2007. This release, as was the previous release, was developed under the direction of the U.S. Department of Energy's Federal Energy Management Program (FEMP). The mission of FEMP is to facilitate the Federal Government's implementation of sound cost-effective energy management and investment practices to enhance the nation's energy security and environmental stewardship. Each of these activities is directly related to achieving requirements set forth in the Energy Policy Acts of 1992 and 2005, the Energy Independence and Security Act (EISA) of 2007, and the goals that have been established in Executive Orders 13423 and 13514 - and also those practices that are inherent in sound management of Federal financial and personnel resources.

The learning objectives of this guide are to:

- Highlight the numerous benefits to metering including those driving the EPAct 2005 legislation as well those used in making the business case for metering.

- Describe the methods and approaches for building-level, panel-level, and end-use metering.

- Achieve an understanding of metering technologies, equipment, and applications.

- Explain the different data communication options for metered data.

- Understand and be able to outline the key elements of a metering plan.

- Highlight the different options for financing metering programs.

The focus of this

guide is to provide

the Federal Energy/

Facility manager

and practitioner with

information and

actions aimed at understanding

metering and

working to achieve

potential savings

and benefits.

- Provide descriptions of applications for meters, data, and data analysis.

- Describe with case studies some immediate metering ideas to generate energy and cost savings.

The focus of this guide is to provide the Federal Energy/Facility manager and practitioner with information and actions aimed at understanding metering and working to achieve the potential savings and benefits.

Based on comments received from the O\&M Working Group, this version of the guide has been revised to make it more useful for the Federal Energy/Facility manager. The major changes include moving information regarding metering economics to Chapter 3, Metering Planning. Information is now included on gas, steam, and water metering as a result of the additional metering requirements set forth in the Energy Independence and Security Act of 2007, and Executive Order 13514 regarding improved water use efficiency and management. Chapter 3 also has been expanded to include a discussion on special metering considerations such as data centers and onsite generation sources and staffing and resource 
requirements that need to be considered in the planning process. In addition, Chapter 2 has been updated to be consistent with the current requirements and new information has been incorporated on smart grid.

The references have been updated to include the most current versions of existing policy documents along with new references tied to new information in the guide. The entire document was revised to increase its readability, and additional case studies and success stories have been included in Chapter 8 . This guide is considered a "living document," so all comments regarding improvement are welcome. Information in Appendix D provides a way that comments and suggestions can be submitted.

This guide consists of eight chapters. The first chapter is an introduction and an overview. Chapter 2 provides the rationale for "Why Metering?” Chapter 3 discusses metering planning, providing key issues and highlighting their importance and covers metering economics and different options for financing metering in the Federal sector. Chapter 4 examines metering approaches and their role in an overall metering program. Chapter 5 looks at the different metering technologies by major utility type: electricity, natural gas, steam, potable water, wastewater, and high-temperature/chilled water. Chapter 6 focuses on metering communications and data storage. Chapter 7 describes the various uses for metered data and options on data analysis via energy information systems. Chapter 8 finishes the guide with some Federal-sector case studies and success stories. Additional information is provided in the appendices. 


\section{Acknowledgments}

This guide is the result of numerous people working to achieve a common goal of highlighting the importance of metering and the resulting opportunities for energy efficiency across the Federal sector. The authors wish to acknowledge the contribution and valuable assistance provided by the staff of the Federal Energy Management Program (FEMP). Specifically, we would like to thank Ab Ream, FEMP O\&M Program Manager, for his leadership and support of this program and development of this document.

The authors would also like to thank the energy and facility staff members of the Federal agencies who have over the years provided us with their ideas and efforts regarding metering needs and practices - all of which have been very helpful in compiling this guide. In particular, we acknowledge the following individuals who supported the development of the case studies: Ed Phillips and Mark Toscano of the Brookhaven National Laboratory, Rich Oswald and Bill Quick at the General Services Administration's Kastenmeier Federal Courthouse, Karen Curran of the General Services Administration Energy Center of Expertise, Greg Leifer of the National Institutes of Health, Adam Savadalena of the Agriculture Research Service of the U.S. Department of Agriculture, Tim Austin, Cedreck Davis, and Rhonda Truitt of the NASA Marshall Space Flight Center, Rick Eshenbach of the U.S. Coast Guard Baltimore Yard, and John Park of Veterans Affairs. In addition, we would like to thank Bill Koran of Portland Energy Conservation, Incorporated (PECI) for his insights on the uses of metered data and Jim Heller of the Naval Facilities Engineering Services Center for his perspectives and experience with metering systems.

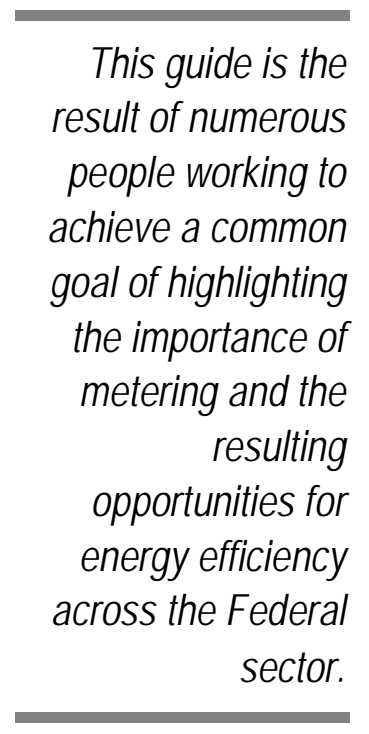

The authors would also like to recognize the various members of the O\&M Working Group of the Interagency Energy Management Task Force that provided invaluable suggestions regarding the reorganization and enhancements required to make the document more useful and timely for facility and energy managers in the Federal sector.

In addition, Kate McMordie Stoughton of Pacific Northwest National Laboratory (PNNL) and Beth Shearer of Beth Shearer and Associates are acknowledged for their inputs, review, and suggestions in the development and production of this guide.

Finally, the authors would like to extend their appreciation to PNNL's document production team - Dave Payson and Kathy Neiderhiser - for the conscientious, team-oriented, and high-quality assistance they brought to this project. 
This page intentionally left blank. 


\section{Acronyms}

AEC

AMR

AOC

API

BAS

BNL

CBECS

CDD

$\mathrm{cfm}$

CHP

CMMS

CRAC

CT

CTS

DC Pro

DoD

DOE

ECM

EIA

EIS

EISA

EO

EPAct

ESPC

EUI

FEDS

FEMP

GAO

GSA

gsf

HDD

HSPD

HVAC

IEEE

IC

IT

LAN

LEED-EB

LEED-NC
Architectural Energy Corporation

automated meter reading

annual operating costs

application program interface

building automation system

Brookhaven National Laboratory

Commercial Buildings Energy Consumption Survey

cooling degree days

cubic feet per minute

combined heat and power

computerized maintenance management system

computer room air conditioning

current transformer

Compliance Tracking System

Data Center Energy Profiler

U.S. Department of Defense

U.S. Department of Energy

energy conservation measure

U.S. Energy Information Administration

energy information system

Energy Independence and Security Act of 2007

Executive Order

Energy Policy Act of 2005

energy savings performance contract

energy-use intensity

Facility Energy Decision System

Federal Energy Management Program

Government Accounting Office

General Services Administration

gross square feet

heating degree days

Homeland Security Presidential Directive

heating, ventilation, and air conditioning

Institute of Electrical and Electronic Engineering

investment costs

information technology

local area network

Leadership in Energy and Environmental Design - Existing Buildings

Leadership in Energy and Environmental Design - New Construction 


\begin{tabular}{|c|c|}
\hline LIPA & Long Island Power Authority \\
\hline $\mathrm{M} \& \mathrm{~V}$ & measurement and verification \\
\hline MCC & motor control center \\
\hline Mcf & thousand cubic feet \\
\hline MSFC & Marshall Space Flight Center (Huntsville, Alabama) \\
\hline NASA & National Aeronautics and Space Administration \\
\hline NFPA & National Fire Protection Association \\
\hline $\mathrm{NIH}$ & National Institutes of Health \\
\hline NIST & National Institute of Standards and Technology \\
\hline NYPA & New York Power Authority \\
\hline $\mathrm{O} \& \mathrm{M}$ & operations and maintenance \\
\hline ODBC & open database connectivity \\
\hline OMETA & Operations, Maintenance, Engineering, Training, and Administration \\
\hline $\mathrm{PF}$ & power factor \\
\hline PIER & Public Interest Energy Resource Program (California) \\
\hline PKI & Public Key Infrastructure \\
\hline PLC & power line communications \\
\hline PNNL & Pacific Northwest National Laboratory \\
\hline Portfolio Manager & ENERGY STAR Portfolio Manager Tool \\
\hline PPE & personal protective equipment \\
\hline psia & pounds per square inch - absolute \\
\hline psig & pounds per square inch - gage \\
\hline PUE & power usage effectiveness \\
\hline PV & present value \\
\hline $\mathrm{RF}$ & radio frequency \\
\hline RMI & radio-magnetic interference \\
\hline RMS & root mean square \\
\hline SCADA & supervisory control and data acquisition \\
\hline scfm & standard cubic feet per minute \\
\hline SIR & savings to investment ratio \\
\hline SQL & structured query language \\
\hline TCP/IP & transmission control protocol/internet protocol \\
\hline THD & total harmonic distortion \\
\hline TOU & time-of-use (pricing) \\
\hline UESC & utility energy service contract \\
\hline UPS & uninterruptible power supply \\
\hline USDA & U.S. Department of Agriculture \\
\hline VA & Department of Veterans Affairs \\
\hline VAR & volt-ampere reactive \\
\hline WAN & wide-area network \\
\hline WBE & whole-building energy \\
\hline XML & extensible markup language \\
\hline
\end{tabular}




\section{Contents}

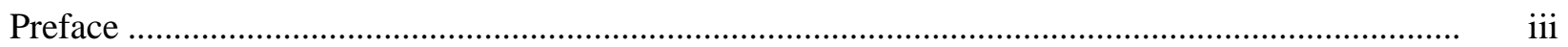

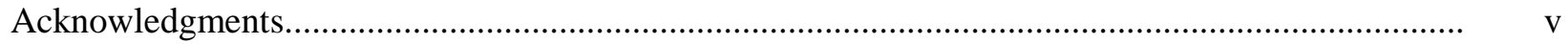

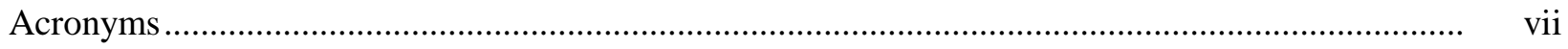

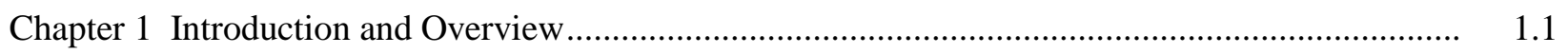

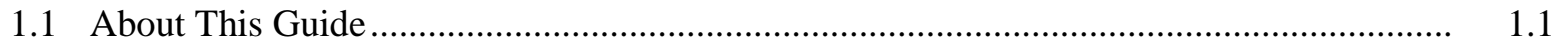

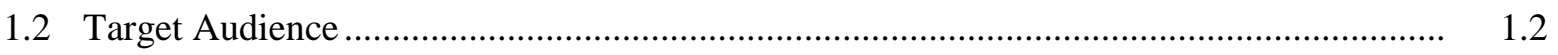

1.3 Organization and Maintenance of the Document......................................................... 1.2

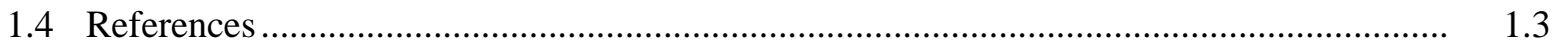

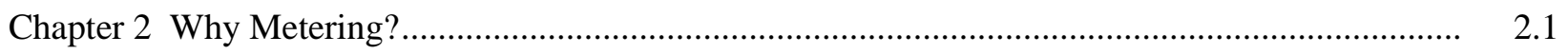

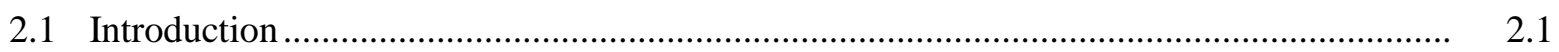

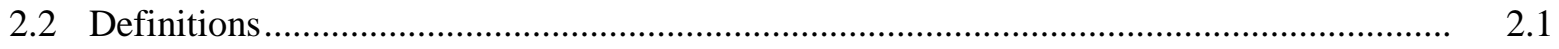

2.3 Motivation and Legislative Drivers................................................................................ 2.2

2.3.1 Legislated Metering Requirements ............................................................... 2.2

2.3.2 Department of Defense Metering Requirements.................................................... 2.3

2.3.3 Other Metering Drivers ……................................................................................. 2.4

2.3.4 Business Case for Metering........................................................................... 2.6

2.3.5 Preparing for the Future ..................................................................................... 2.7

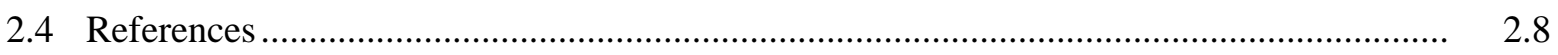

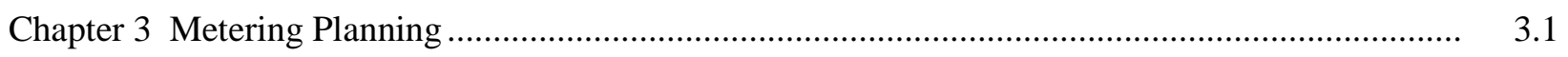

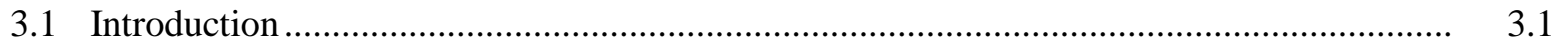

3.2 The Need for a Metering Plan .....................................................................................

3.3 Steps to Consider in Your Metering Plan...................................................................... 3.2

3.3.1 Establish Program Goals, Objectives, and Risk Mitigation .................................... 3.2

3.3.2 Identify Needs to Support Selected Analysis Approaches ...................................... 3.4

3.3.3 Develop and Apply Evaluation Criteria ................................................................ 3.5

3.3.4 Implementation, Design, and Installation............................................................ 3.6

3.3.5 Performance Validation and Persistence ........................................................... 3.7

3.3.6 Special Metering Considerations.................................................................... 3.8

3.3.7 Staffing and Resource Requirements ................................................................ 3.8

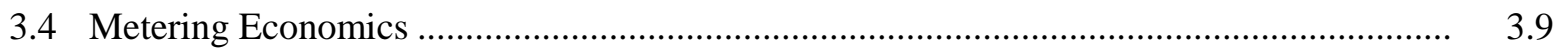

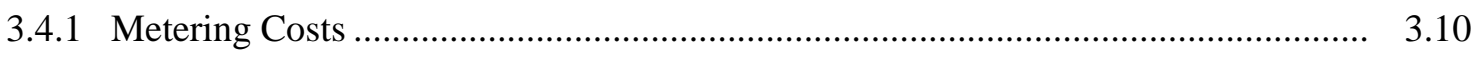

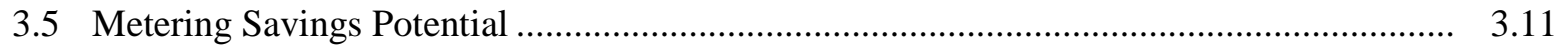

3.5.1 Cost Justification Methodology …................................................................... 3.12

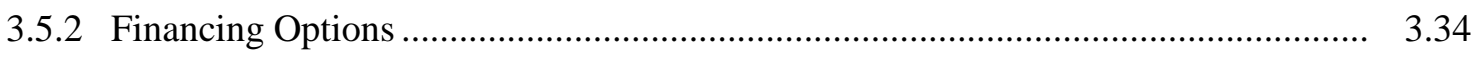

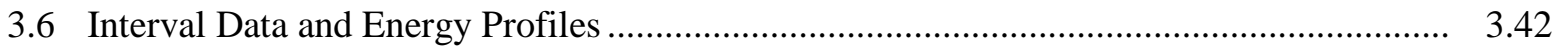


3.7.1 Data Collection.

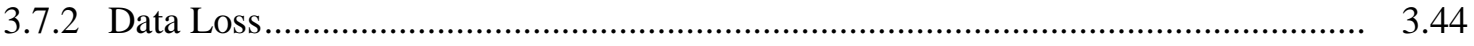

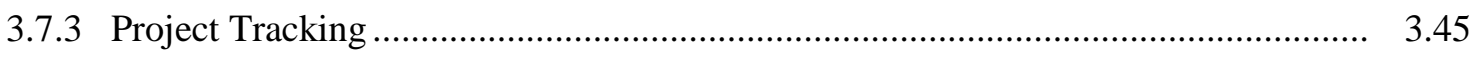

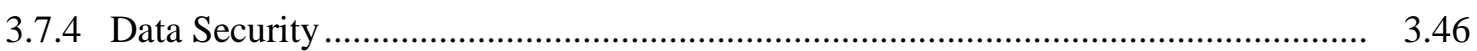

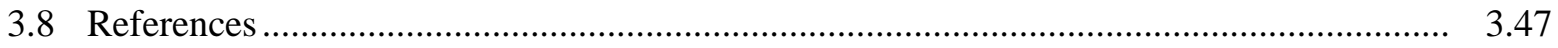

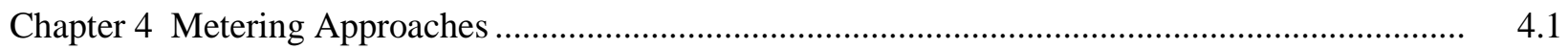

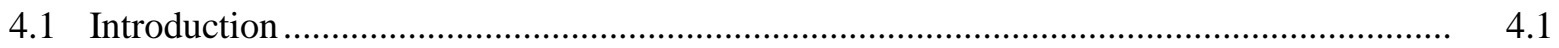

4.2 Generic Approaches ................................................................................................

4.2.1 One-Time/Spot Measurements....................................................................... 4.1

4.2.2 Run-Time Measurements .............................................................................. 4.2

4.2.3 Short-Term Measurements/Monitoring ................................................................. 4.2

4.2.4 Long-Term Measurements/Monitoring ............................................................... 4.3

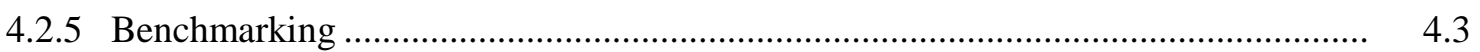

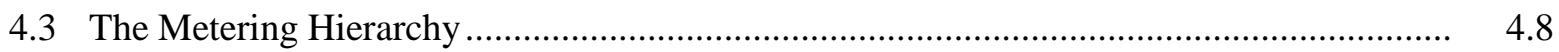

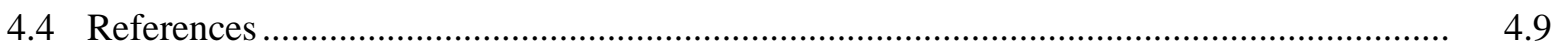

Chapter 5 Metering Technologies................................................................................... 5.1

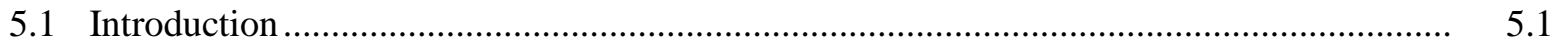

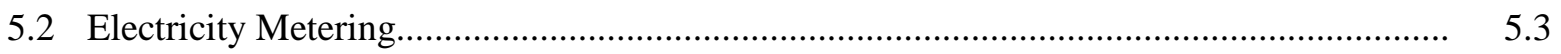

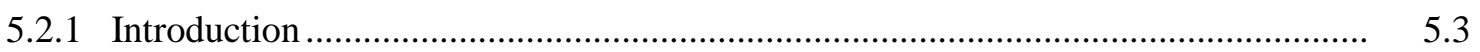

5.2.2 Common Electrical Metering Terms ….................................................................. 5.3

5.2.3 Electricity Metering Technologies ..................................................................... 5.4

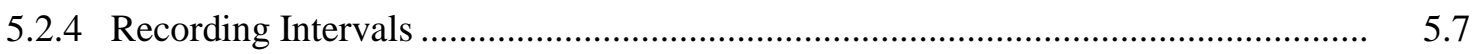

5.2.5 Electric Metering Maintenance ..................................................................... 5.7

5.2.6 Electric Meter Data Output/Communications Considerations ............................... 5.7

5.2.7 Advanced Electric Meter Specification Considerations .......................................... 5.8

5.2.8 Advanced Electric Meter Selection Considerations ................................................. 5.8

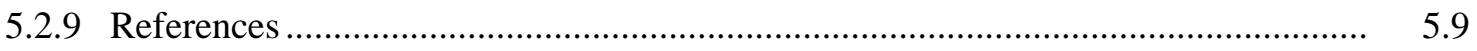

5.3 Fluid Metering - Natural Gas............................................................................. 5.11

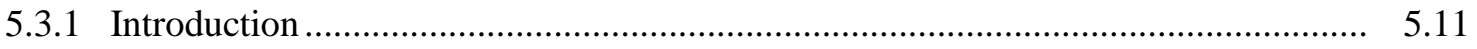

5.3.2 Natural Gas Metering Technologies................................................................. 5.11

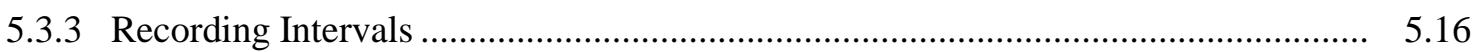

5.3.4 Natural Gas Meter Maintenance...................................................................... 5.17

5.3.5 Natural Gas Metering Data Output/Communications Considerations .................... 5.18

5.3.6 Natural Gas Meter Specification Considerations .................................................. 5.19

5.3.7 Natural Gas Meter Selection Criteria .................................................................... 5.19

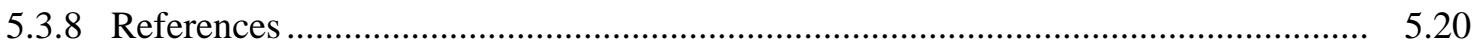


5.4.1 Introduction

5.4.2 Steam Metering Technologies........................................................................... 5.21

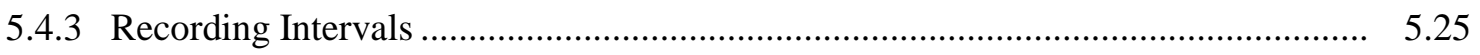

5.4.4 Steam Meter Maintenance................................................................................ 5.25

5.4.5 Steam Metering Data Output/Communications Options....................................... 5.26

5.4.6 Steam Meter Specification Considerations ....................................................... 5.26

5.4.7 Steam Meter Selection Criteria ........................................................................... 5.27

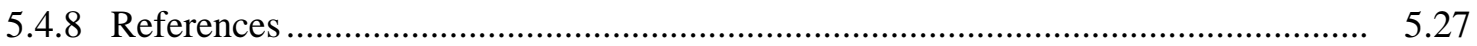

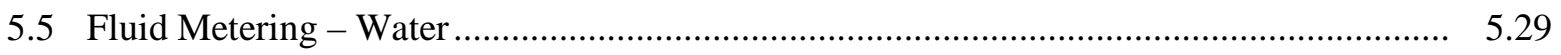

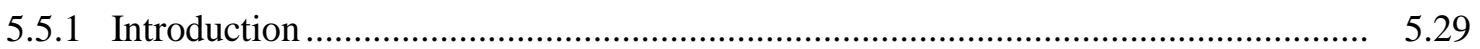

5.5.2 Water Metering Technologies .......................................................................... 5.29

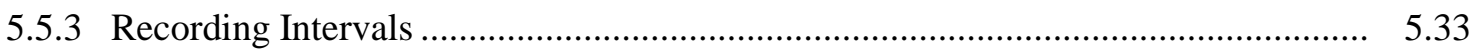

5.5.4 Water Meter Maintenance ................................................................................. 5.33

5.5.5 Water Metering Data Output/Communications Considerations............................... 5.35

5.5.6 Water Meter Selection Considerations ................................................................. 5.35

5.5.7 Water Meter Selection Criteria........................................................................... 5.36

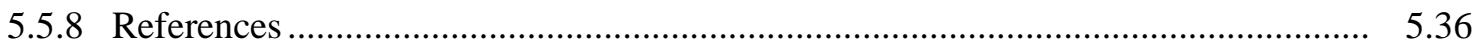

5.6 Fluid Metering - High-Temperature Hot Water/Chilled Water........................................ 5.37

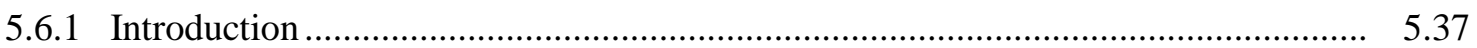

5.6.2 High-Temperature Hot Water/Chilled Water Technologies ................................... 5.37

5.6 .3 Recording Intervals .................................................................................... 5.42

5.6.4 High-Temperature/Chilled Water Meter Maintenance ........................................ 5.42

5.6.5 High-Temperature/Chilled Water Metering Data Output/Communications Considerations .................................................................................................. 5.44

5.6.6 High-Temperature/Chilled Water Meter Selection Considerations ......................... 5.44

5.6.7 High-Temperature Hot Water/Chilled Water Meter Selection Criteria ................... 5.45

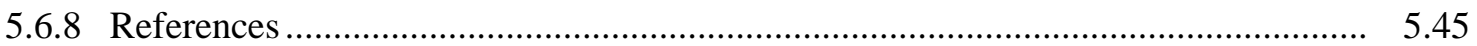

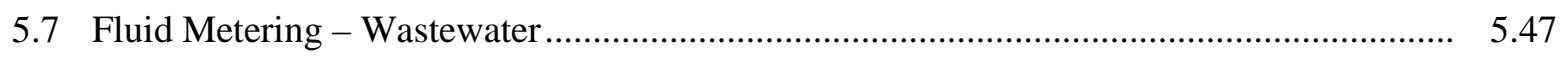

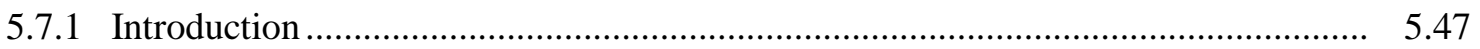

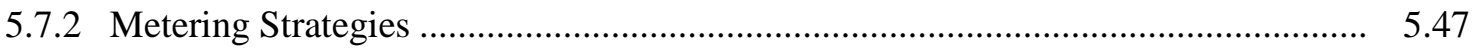

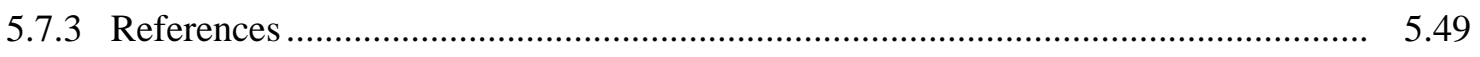

5.8 Metering of Onsite Power Generation...................................................................... 5.51

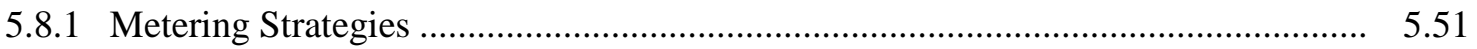

5.8 .2 Metering Applications............................................................................... 5.52

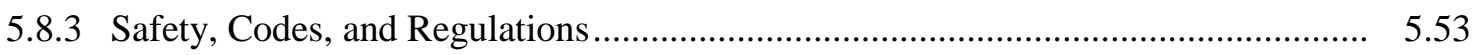

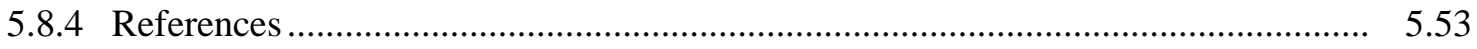

Chapter 6 Metering Communications and Data Storage........................................................... 6.1

6.1 Traditional Communications Options - Non-Automated ............................................... 6.1 
6.2 Modern Metering Communications - Automated Meter Reading ................................... 6.2

6.3 Data Storage Software...................................................................................... 6.4

6.3.1 Data Storage Specification Considerations …............................................................ 6.4

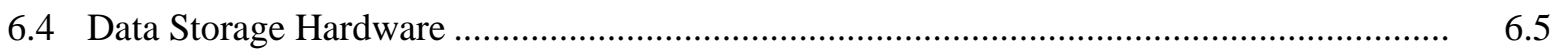

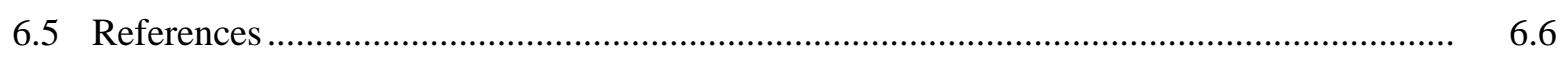

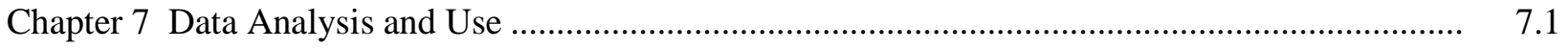

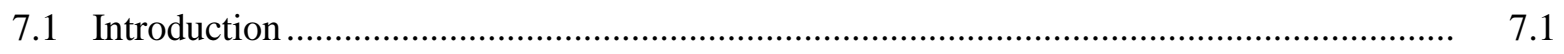

7.1.1 Data Analysis and Energy Information Systems .................................................. 7.1

7.2 Uses for Data - Metering Applications .......................................................................... 7.5

7.3 Working with Data - Examples from the Field ............................................................ 7.8

7.3.1 Interval Data for Efficiency Opportunity Identification......................................... 7.8

7.3.2 Building Benchmarking ....................................................................................

7.3.3 Operational Efficiency Identification ............................................................. 7.10

7.3.4 Peak Demand Identification/Reduction............................................................... 7.11

7.3.5 Operational Efficiency Opportunity Using Data Logger Data to Validate Boiler Operation .................................................................................................. 7.12

7.3.6 Measurement and Verification of New Lighting Technology ............................... 7.12

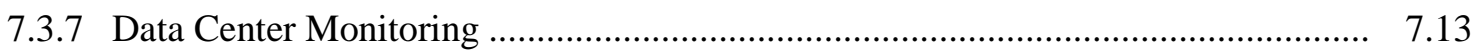

7.3.8 Overcoming Common Metering Challenges....................................................... 7.16

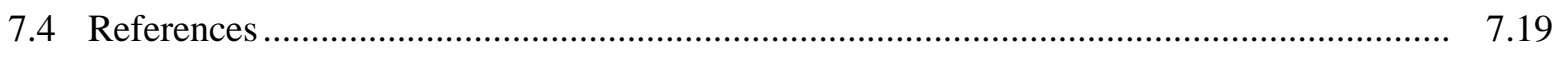

Chapter 8 Federal Sector Case Studies/Success Stories .........................................................

8.1 Electric Metering Program at Brookhaven National Laboratory …................................... 8.1

8.2 General Services Administration's Kastenmeier Federal Courthouse ............................... 8.3

8.3 General Services Administration’s Enterprise- Based Metering System........................... 8.6

8.4 National Institutes of Health Headquarters ..................................................................... 8.8

8.5 U.S. Department of Agriculture: Western Regional Research Center .............................. 8.11

8.5.1 Metering Objectives ...................................................................................... 8.11

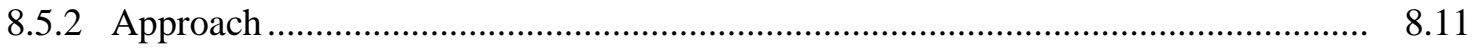

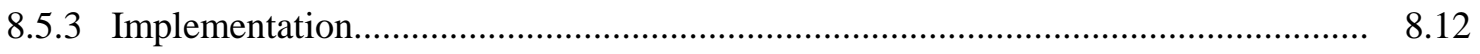

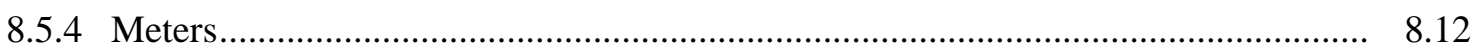

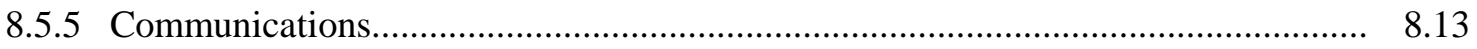

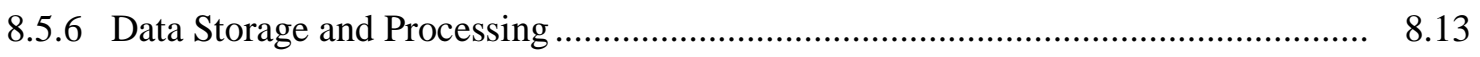

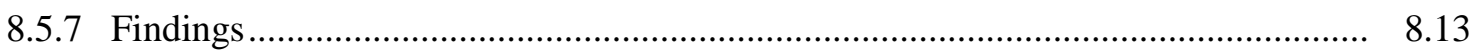

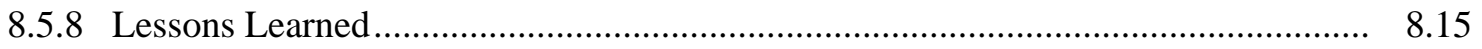

8.6 NASA Marshall Space Flight Center Metering Program.............................................. 8.16

8.6.1 Program Description .................................................................................. 8.16

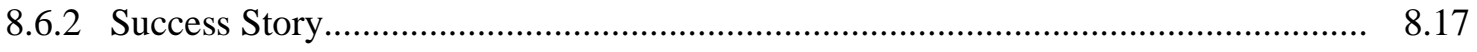

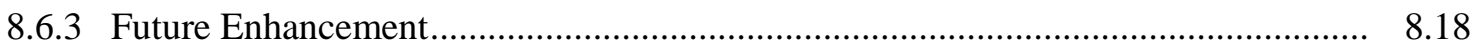


8.7 ....................................S. Coast Guard - Baltimore Yard Electric Metering Program

8.7.1 Metering Objectives

8.7.2 Approach.

8.19

8.7.3 Implementation...

8.20

8.7.4 Findings

8.21

8.7.5 Lessons Learned

8.22

8.8 Department of Veterans Affairs Advanced Metering Program......................................... 8.22

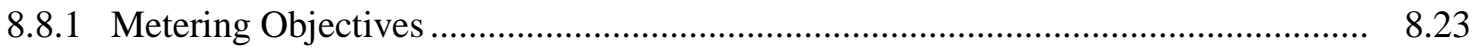

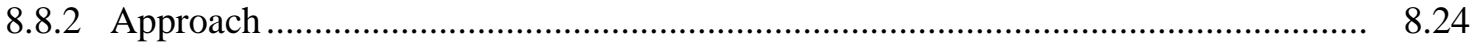

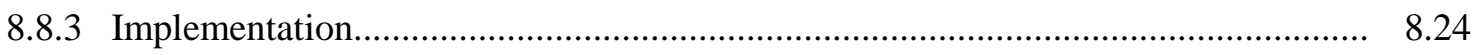

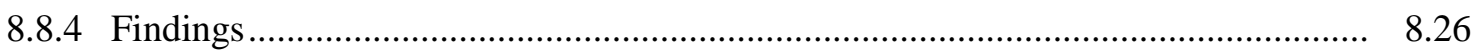

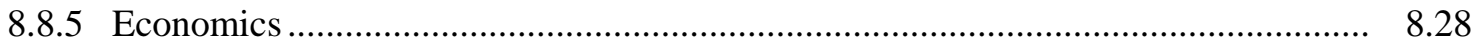

8.8.6 Lessons Learned .................................................................................... 8.28

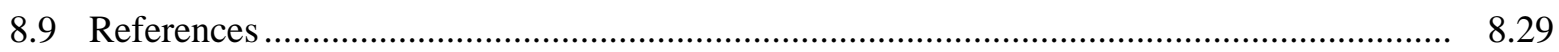

Appendix A - Glossary of Common Terms …................................................................................ A.1

Appendix B - Legislative Requirements ............................................................................. B. 1

Appendix C - Metering Codes and Standards .................................................................... C.1

Appendix D - Suggestions for Additions or Revisions ........................................................... D. 


\section{Figures}

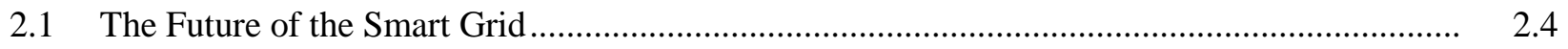

3.1 Metering Planning Process................................................................................................

3.2 Minimum Electric Bill versus Annual Percentage Savings ….................................................. 3.14

Equipment Nameplate.......................................................................................... 3.16

3.4 Savings Graph versus Gas Consumption @ 1 Percent and 5 Percent Savings ........................... 3.17

3.5 Case 1 - Gas Use of 40,000 Mcf versus SIR @ 1 Percent Savings and \$2,000 Installed

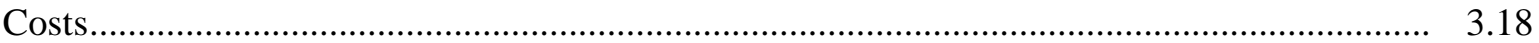

3.6 Case 2 - Gas Use of 40,000 Mcf versus SIR @ 1 Percent Savings and \$4,000 Installed

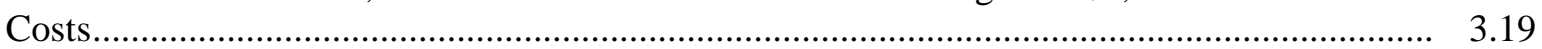

3.7 Case 3 - Gas Use of 40,000 Mcf versus SIR @ 1 Percent Savings and \$6,000 Installed

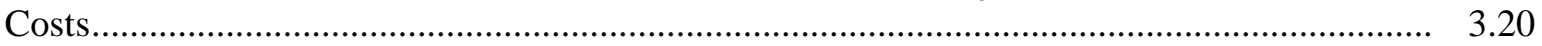

3.8 Central Steam Plant Schematic ....................................................................................... 3.23

3.9 Steam Pressure Drop................................................................................................ 3.24

3.10 Savings Graph versus Annual Steam Consumption @ 1 Percent and 5 Percent Savings........... 3.25

3.11 Case 1 - Steam Use of 12.1 klbs versus SIR Ratio @ 1 Percent Savings, \$2,000

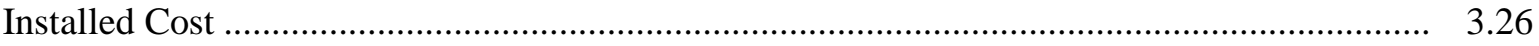

3.12 Case 3 - Steam Consumption of 12.1 klbs versus SIR Ratio @ 1 Percent Savings and \$6K Installed Costs ........................................................................................................ 3.27

3.13 Cost-Effectiveness of Positive Displacement Meters ........................................................... 3.31

3.14 Cost-Effectiveness of Turbine Meters …............................................................................ 3.32

3.15 Cost-Effectiveness of Vortex Shedding Meters .................................................................. 3.32

3.16 Cost-Effectiveness of Portable Ultrasonic Meters .................................................................

3.17 Cost-Effectiveness of Permanent Ultrasonic Meters ............................................................ 3.34

3.18 The Metering Financing Hierarchy .............................................................................. 3.35

3.19 The National Institutes of Health in Bethesda, Maryland, is using an ESPC to install over 350 meters across the complex .................................................................................... 3.41

3.20 Typical Commercial Building Daily Electric Load Profile .................................................... 3.43

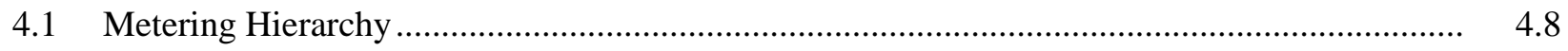

5.1 Typical Natural Gas Diaphragm Meter.......................................................................

5.2 Typical Natural Gas Rotary Flow Meter............................................................................. 5.12

5.3 Rotary Flow Meter Function........................................................................................... 5.13

5.4 Typical Orifice Meter................................................................................................... 5.14

5.5 Typical Venturi Meter................................................................................................... 5.14

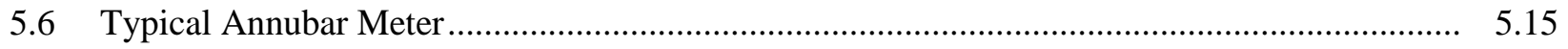

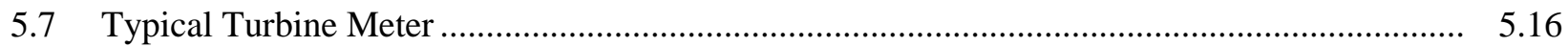

5.8 Typical Vortex Shedding Meter.................................................................................. 5.17

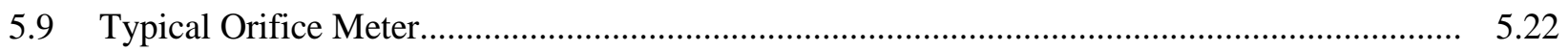

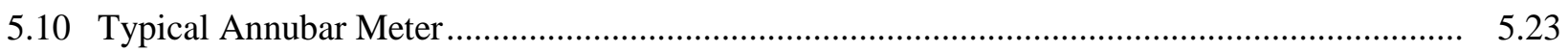




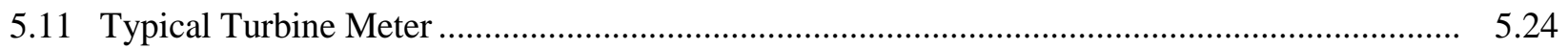

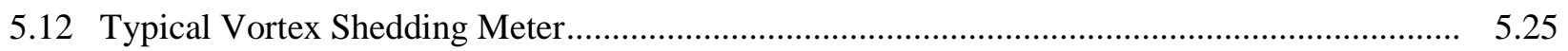

5.13 Typical Orifice Meter..................................................................................................... 5.30

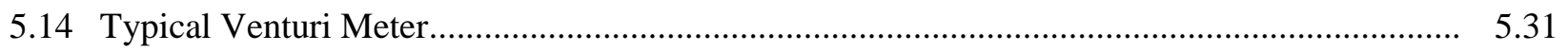

5.15 Typical Turbine Meter ….............................................................................................. 5.32

5.16 Typical Vortex Shedding Meter...................................................................................... 5.32

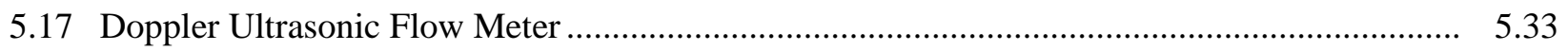

5.18 Transit Time Ultrasonic Flow Meter.................................................................................. 5.33

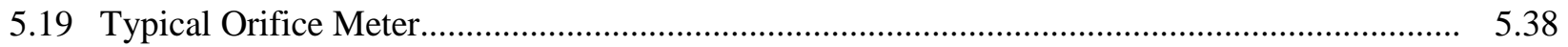

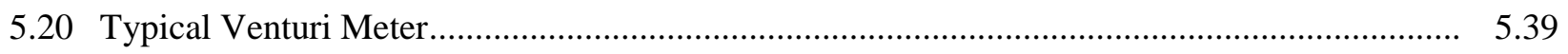

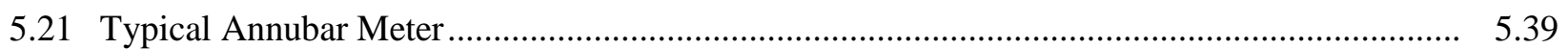

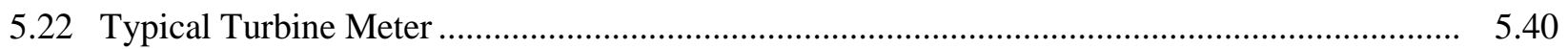

5.23 Typical Vortex Shedding Meter................................................................................ 5.41

5.24 Doppler Ultrasonic Flow Meter ................................................................................... 5.41

5.25 Transit Time Ultrasonic Flow Meter................................................................................... 5.42

5.26 Typical Photovoltaic Array Connection and Metering Scheme.............................................. 5.52

7.1 Daily Demand Profile - Nighttime Temperature Setback Disabled ....................................... 7.8

7.2 Daily Demand Profile - Nighttime Setback Enabled...................................................................

7.3 Building Benchmarking ..........................................................................................

7.4 Building Water-Use Interval Data - Suspicious Pattern...................................................... 7.10

7.5 Building Water-Use Interval Data - Verification of Water Leak ........................................... 7.10

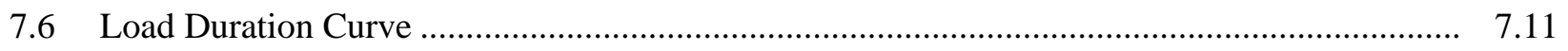

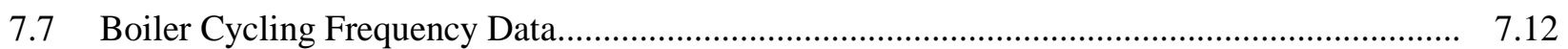

7.8 Measurement and Verification of Lighting Demonstration ................................................. 7.13

7.9 Comparative Annual Energy Costs of Different Facility Types ….......................................... 7.13

7.10 Typical Data Center ................................................................................................. 7.14

8.1 Monthly Summary of Scheduled vs. Actual Site Electric Demand Loads ............................... 8.2

8.2 Installed Wireless Monitoring System for WBE-Based System............................................. 8.4

8.3 Sample Screen Capture for a Generic Building Showing an Alarm or High-Energy Using Condition ................................................................................................................. 8.4

8.4 Data from the Monitoring Device Provides a Comparison of 2 Days of Electricity Use for Sunday, January 15, 2006, and Monday, January 16, 2006 ........................................ 8.5

8.5 Summary of Current Conditions at a GSA Building in Waltham, Massachusetts..................... $\quad 8.7$

8.6 Chiller Water Metering at NIH ......................................................................................... 8.9

8.7 Compressed Air Meter Applications at the NIH..................................................................

8.8 Total Site Steam General vs. Single Building Steam Demand .............................................. 8.10

8.9 Sample of Meters Shown in Graphical User Interface.......................................................... 8.13

8.10 Time Series Power Profiles of Laboratory and Greenhouse Facilities for the Period Friday, April 15, 2011, through Wednesday, April 20, 2011 
8.11 Three Phase Amperages - Phases A, B, C, and Average and System Neutral for Office Wing

8.12 Time Series Power Factor Profiles of Service Main, Admin Wing, Greenhouse,

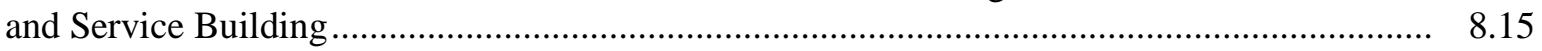

8.13 Winter Time CCWP Water Consumption FY 2007 through FY 2011 ................................... 8.17

8.14 The Coast Guard Yard at Baltimore, Maryland ................................................................ 8.18

8.15 Resource Metering Configuration at Three Coast Guard Installations ................................... 8.20

8.16 Air Compressor Operation "Before” and “After” Metering Program Implementation .............. 8.21

8.17 Warehouse Lighting Operation "Before” and "After” Metering Program Implementation ....... 8.21

8.18 Electric Welder Operation “Before” and “After” Metering Program Implementation.............. 8.22

8.19 Veterans Administration Medical Center, Palo Alto, California ............................................... 8.23

8.20 Schematic of Veterans Affairs Pilot-Phase Metering Project ................................................. 8.25

8.21 Schematic of Veterans Affairs Phase 2: National Electric Metering Project........................... 8.26

\section{Tables}

$2.1 \quad$ Federal Metering Requirements Summary …................................................................... 2.5

$3.1 \quad$ Example Electric Data Requirements ….............................................................................. 3.5

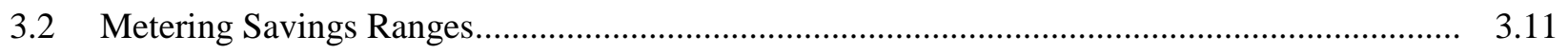

3.3 Current Range of Natural Gas Prices ............................................................................ 3.15

5.1 Common Natural Gas Metering Technologies and Key Criteria............................................ 5.19

5.2 Common Steam Metering Technologies and Key Criteria .................................................... 5.27

5.3 Common Water Metering Technologies and Key Criteria .................................................. 5.36

5.4 Common Water Metering Technologies and Key Criteria ................................................... 5.45

7.1 Load Duration Curve Data .............................................................................................. 7.11

7.2 Benchmark Data Center Power Usage Effectiveness ......................................................... 7.16 


\section{Chapter 1 Introduction and Overview}

The purpose of this guide is to provide you, the Facility/Energy manager and practitioner, with useful information about energy and resource metering, the relevant metering technologies, communications, applications for data, and ideas for energy and cost savings. In addition, this guide assists in the implementation of metering requirements in accordance with the Energy Policy Act of 2005 (EPAct 2005) and the Energy Independence and Security Act of 2007 (EISA 2007) and any metering that would be associated with the requirements of Executive Order 13514 Federal Leadership in Environmental, Energy, and Economic Performance (EO 13514). To make this guide useful and to reflect your needs and concerns, the authors met with Facility and Energy managers via Federal Energy Management Program (FEMP) workshops and annual conferences. In addition, the authors conducted literature searches and contacted numerous vendors and industry experts. The information and case studies that appear in this guide resulted from these activities.

It needs to be stated at the outset that this guide is designed to provide information on effective metering strategies as they apply to systems and equipment typically found at Federal facilities. This guide is not designed to provide the reader with step-by-step procedures for installing or connecting metering equipment. Rather, this guide first directs the user to qualified installation entities and the manufacturer's specifications and recommendations. In no way should the recommendations in this guide be used in place of manufacturer's recommendations. The recommendations in this guide are designed to supplement those of the manufacturer - or, as is all too often the case, provide guidance for systems and equipment for which all documentation has been lost.

As a rule, this guide will first defer to the manufacturer's recommendations on equipment metering installation and operation.

Actions and activities recommended in this guide should only be attempted by

STOP trained and certified personnel. If such personnel are not available, the actions recommended here should not be initiated.

This guide is designed to serve as a resource for facility and energy management and technical staff.

\subsection{About This Guide}

This guide is designed to serve as a resource for facility and energy management and technical staff. It does not try to represent the universe of metering-related material. Rather, it attempts to:

- Provide needed background information on why metering is important and the potential for savings from properly executed metering programs.

- Define the major approaches to metering and provide guidance on the structure of an effective metering program.

- Provide information on state-of-the-art metering and communications technologies. 


A competent metering
program requires the
participation of staff
from five well-defined
areas within the
overall facilities
organization - these
are Operations,
Maintenance,
Engineering, Training,
and Administration.

- Highlight the more common and applicable uses for metered data as they apply to the Federal sector.

- And finally, identify information sources and contacts to assist you in getting your job done.

\subsection{Target Audience}

Facility and energy managers, practitioners, and technical staff represent the prime focus of this document. However, a competent metering program requires the participation of staff from five well-defined areas within the overall facilities organization - these are Operations, Maintenance, Engineering, Training, and Administration (OMETA). For more information on OMETA, see the O\&M Best Practices Guide (FEMP 2010). While a given site may not have all five of these areas as separate entities, these functions are provided for within the organization. It is these staff that are the target audience of this guide.

A successful metering program requires cooperation, dedication, and participation at all levels and cannot succeed without everyone involved understanding the basic principles and supporting the efforts to acquire technically sound data regarding resource use within buildings or associated with delivery of an energy resource.

\subsection{Organization and Maintenance of the Document}

This document represents Release 2.0 of Metering Best Practice Guide: A Guide to Achieving Utility Resource Efficiency. The initial release of this document occurred in October 2007. The expectation is that future releases will be made as new metering technologies, procedures, and requirements are developed and employed.

It is the intention of the authors to update this guide periodically as new metering technologies and procedures are developed and employed.
This guide consists of eight chapters. The first chapter is an introduction and an overview. Chapter 2 provides the rationale for "Why Metering?” Chapter 3 discusses metering planning, providing key issues, and highlighting their importance. Chapter 4 examines metering approaches and their role in an overall metering program. Chapter 5 looks at the different metering technologies by major utility type; electricity, natural gas, steam, high temperature/ chilled water, and potable water. Chapter 6 focuses on metering communications and data storage. Chapter 7 describes the various uses for metered data and options on data analysis via energy information systems. Chapter 8 finishes the guide with Federal-sector case studies and success stories.

The metering facility management environment is in a constant state of evolution and the technologies and vocabularies are ever expanding. Therefore, a glossary of terms is presented in Appendix A. Appendix B provides a copy of Section 103 of the Energy Policy Act of 2005 and Section 434(b) of the Energy Independence and Security Act of 2007. Appendix C includes applicable codes and standards as related to metering equipment and installations. And finally, Appendix D is a form that can be used to submit suggestions or revisions to this guide. 
This document was designed and organized to provide useful information. All inputs are welcome to help keep it current. Please feel comfortable to make suggestions for changes, additions, or deletions using the form found in Appendix D.

\subsection{References}

Energy Independence and Security Act of 2007 (EISA 2007). 2007. Public Law 110-140, as amended, Section 434(b).

Energy Policy Act of 2005 (EPAct 2005). 2005. Public Law 109-58, as amended, Section 103, Energy Use Measurement and Accountability, Section 543 (42 USC 8253), (e) Metering of Energy Use.

Executive Order (EO) 13514. 2009. Federal Leadership in Environmental, Energy, and Economic Performance. Signed October 8, 2009.

Federal Energy Management Program (FEMP). 2010. Operations \& Maintenance Best Practices: A Guide to Achieving Operational Efficiency, Release 3.0, prepared by the Pacific Northwest National Laboratory for the Federal Energy Management Program, U.S. Department of Energy, Washington, D.C. Available at: http://www1.eere.energy.gov/femp/pdfs/omguide_complete.pdf. 
This page intentionally left blank. 


\section{Chapter 2 Why Metering?}

\subsection{Introduction}

Energy managers have long known the value of energy-use data. And with recent advances in energy-use metering - increased functionality at lower costs - obtaining these data in a cost-effective manner is now becoming a standard practice. Whether energy managers are trying to comply with legislated and mandated metering requirements, or looking to apply accepted building management best practices such as utility bill verification or benchmarking, today's metering technologies can provide the information needed to meet energy goals, save money, and improve their building operations.

\subsection{Definitions}

Metering of electricity (and other utilities) has seen an increase in interest, application, and technology advance in both the private (i.e., utility) and the public (i.e., Federal) sectors. Two significant drivers of this heightened interest are the ongoing modernization of the nation's electric infrastructure with the move toward the smart grid and smart meters. On the Federal side, the drivers are the legislative mandates for metering of Federal buildings (see Section 2.3 below) and the incorporation of advanced meters within Federal buildings.

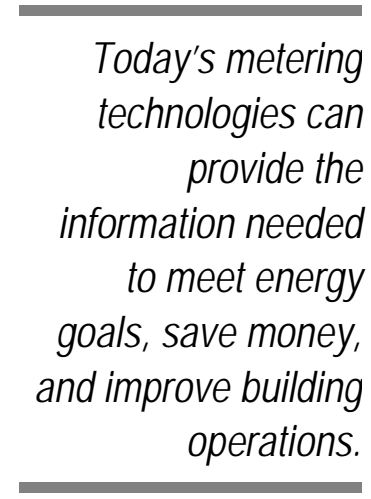

While these two activities are on parallel paths toward providing more and better information to their respective constituents ("smart meters” for utilities and "advanced meters” for Federal/other facility operators), at some point it is expected that these two paths will intersect - affording the sharing of information for mutual benefit.

While technology inevitably changes, the definitions below are somewhat fixed in time (DOE 2006). As such, these definitions try to capture technology and capability as it exists today, while acknowledging future advancements are expected.

Advanced Meters. Typically developed with a customer or end-use focus (e.g., functional to the Facility/Energy Manager) and have the capability to measure and record interval data and communicate the data to a remote location in a format that can be easily integrated into an advanced metering system. EPAct 2005 Section 103 requires at least hourly interval resolution and at least daily data collection ability. Advanced meters do have variance in features depending on the utility they are serving:

- In the case of electricity, advanced meters offer an increasing array of measurements, recording intervals, and communications options. For Federal compliance, measurements of power $(\mathrm{kW})$ and energy ( $\mathrm{kWh}$ ) are important. Beyond these, the options expand to potentially useful diagnostics with the monitoring voltages, currents, power factor, power quality, and statistical capabilities. These metrics are available in a variety of output options (milliamp, voltage, Modbus, and pulse) and an ever-increasing communications suite.

- In the case of other utilities (gas, steam, and water), advanced meters focus on the ability to record interval data and communicate these data to those responsible for the management of the resource. 
Smart Meters. Developed largely to meet utility needs and the increasingly sophisticated distribution grid, smart meters are focused on the "whole building" element of metering. In many cases, these meters satisfy the legislative mandates of interval data and remote communications, however, beyond that, their capabilities focus on utility infrastructure needs and grid interconnect. Variability in smart meters across utility applications includes:

- In the case of electricity, smart meters focus on providing the utility (and ultimately the consumer) with time-differentiated energy use. A large driver in smart meter technology is a need to capture and report interval energy use for demand response programs and expected time-of-use pricing/rate structures. Additional utility applications include remote meter reading applications and service connection/disconnection abilities.

- Smart meter application with the other utilities is similar to the advanced meters with the focus on an ability to record interval data and communicate these data as required.

Advanced Metering System. A system that collects time-differentiated energy usage data from advanced meters via a network system on either an on-request or defined schedule basis. The system is capable of providing usage information on at least a daily basis and can support desired features and functionality related to

The importance of metering can be summed up in the Energy Manager's maxim:

If you don't collect it - you can't measure it. If you don't measure it - you can't manage it. energy-use management, procurement, and operations.

Standard meters. Electromechanical or solid state meters that cumulatively measure, record, and store aggregated usage data that are periodically retrieved for use in customer billing or energy management. Meters that are not advanced meters are standard meters.

See Appendix A, Glossary of Common Terms, for additional advanced metering terms and definitions.

\subsection{Motivation and Legislative Drivers}

There are several legislative acts that require metering of energy resources at Federal facilities. The specific metering requirements in these acts are based on the idea that the data provided by the meters (and its subsequent analysis and actions) will greatly help sites reduce their energy use and costs in a costeffective manner. Beyond legislation, motivation to consider the application of meters is also provided in agency policy for energy efficiency and/or sustainable operations, as well as through the efforts of the local utility companies looking to better manage or reduce their customer loads on an increasingly constrained electric grid. Finally, while the application of meters will not be cost-effective for all utilities in all locations, its consideration for all applications is necessary for sites looking to improve their operations.

\subsubsection{Legislated Metering Requirements}

Section 103 of the EPAct (2005) (Public Law 109-58) requires that "all Federal buildings shall, for the purposes of efficient use of energy and reduction in the cost of electricity used in such buildings be metered ... to the maximum extent practicable.” This requirement of law is the driving force behind the ongoing efforts of Federal agencies to meter their electric use. The primary metering requirements 
established in Section 103 of EPAct 2005, Energy Use Management and Accountability, ${ }^{1}$ are summarized by these key points:

- By October 1, 2012, all Federal buildings will be metered for electricity subject to determination of practicability.

Stakeholders involved in the development of the guidelines required by paragraphdetermined practicability include: installation of metering and advanced metering wherever feasible; capability of providing useful data that lead to improved energy management practices or operations and maintenance improvements resulting in Metering Beyond the EPAct 2005 Requirements As Federal sites are implementing and expanding their metering programs, they are encouraged to consider meter applications above the EPAct 2005 and EISA 2007 requirements. In addition to metering building electricity use, natural gas, and steam, also consider metering (where cost-effective) water and compressed air. Also consider metering beyond the building level and instead look at sub-metering consumption by tenants within buildings and/or at the equipment level to support optimization activities. energy and/or energy-related cost savings; sensible application of metering technology that is cost-effective based on a 10-year simple payback, an assumed annual savings of 2 percent or higher, and use of the metering data to implement projects. The definition of the term buildings should be considered the same as for the annual energy reporting and also include industrial or process applications.

- Advanced metering devices that provide interval data on at least a daily basis will be used subject to practicability.

- Metered data shall be incorporated into a Federal tracking system and made available to Federal facility managers.

The complete text of EPAct 2005 Section 103 can be found in Appendix B, Legislative Requirements.

By October 1, 2012, all Federal buildings will be metered for electric use subject to determination of practicability.
Energy Independence and Security Act of 2007. Among other requirements, EISA (2007) further strengthens the metering requirements of EPAct 2005 and the National Energy Conservation Policy Act with the following language:

- Not later than October 1, 2016, each agency shall provide for equivalent metering of natural gas and steam, in accordance with guidelines established by the Secretary under paragraph (2) of section 543(e)(1) of the National Energy Conservation Policy Act (42 USC 8253(e)(1)).

- In addition to energy, the metering of water is encouraged to obtain data to support the water intensity reduction goals as outlined in EISA 2007.

\subsubsection{Department of Defense Metering Requirements}

The facilities owned and operated by the U.S. Department of Defense (DoD) account for approximately two-thirds of the total facility energy used by Federal buildings in 2005. In an effort to better manage its energy use and costs, DoD Instruction 4170.11, Installation Energy Management (DoD 2009), was issued. Paragraph 3.d(2), Metering, establishes the expanded metering requirements for DoD buildings to

\footnotetext{
${ }^{1}$ The metering requirements of EPAct 2005 amended Section 543 of the National Energy Conservation Policy Act (42 USC 8253).
} 
include metering of electricity, natural gas, and water on appropriate facilities (those where "metering would be cost-effective and practical as a management enhancement tool”), and the metering of steam at steam plants. DoD also requires that meters be installed on all new construction and utilities systems renovation projects exceeding \$200,000. Chapter 10 of the Department of Defense Energy Manager's Handbook (DoD 2005) provides additional clarification for sites on meeting the requirements of the Instruction.

Table 2.1 presents a summary of the recent legislative and metering requirements by establishing authority.

\subsubsection{Other Metering Drivers}

Increasing meter functionality, declining costs of meters, and a growing recognition of the value of metered data also contribute to the expanded use of energy/utility metering. Examples include

- EPAct 2005 Section 1252, Smart Meters, requires states to investigate requiring the utilities to offer time-based rates to their customers. Electric utilities directed to offer time-based rates will be required to provide the customers on the time-based rate with an advanced meter. (See Section 2.3.5 for more details.)

- Smart Grid - In the simplest of terms, a smart grid provides electrical energy from suppliers to consumers accompanied by two-way digital communication. It is this communication ability that affords the grid its intelligence. Figure 2.1 provides a graphical representation of the smart grid.

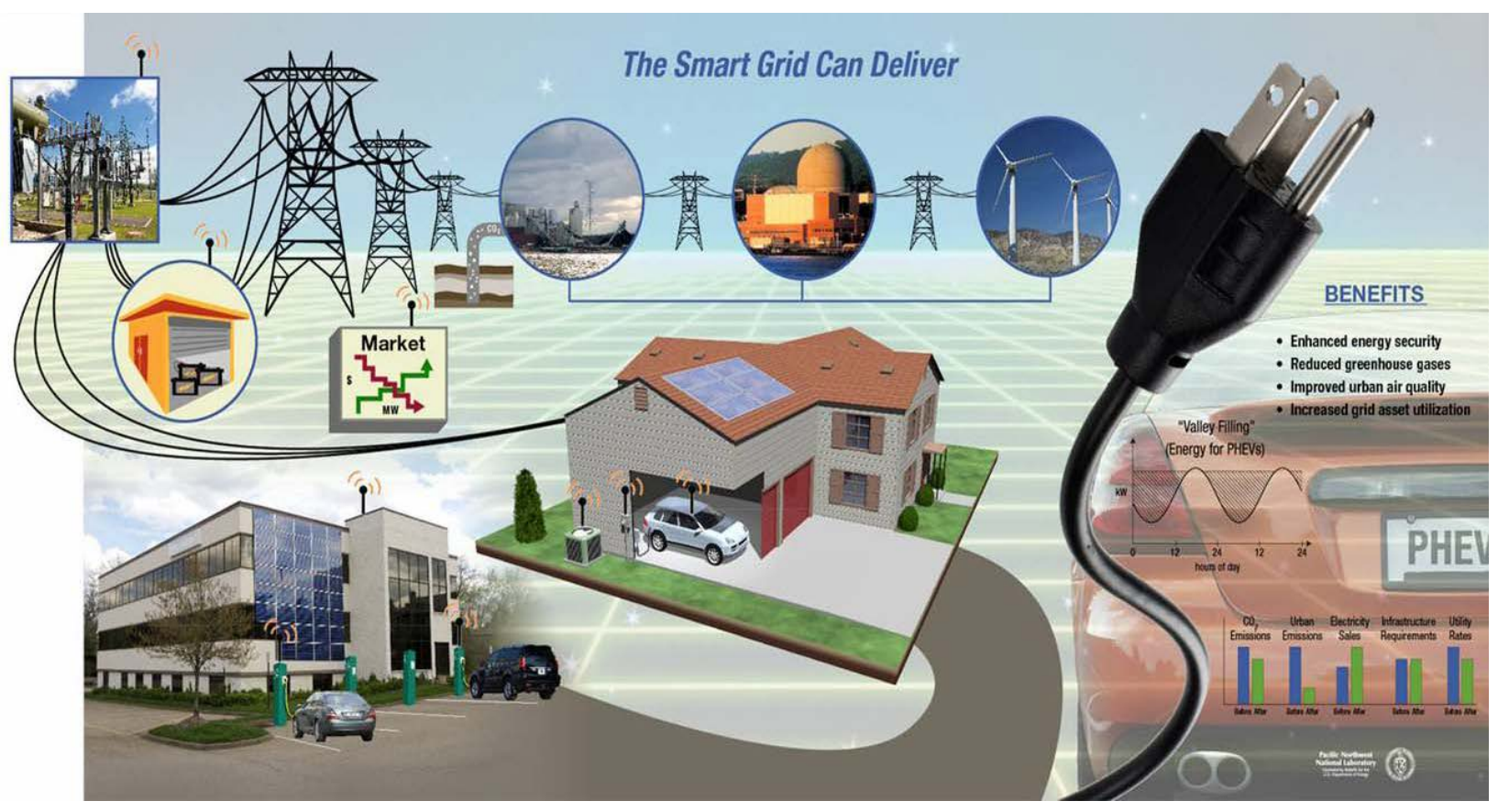

Figure 2.1. The Future of the Smart Grid (DOE 2007) 


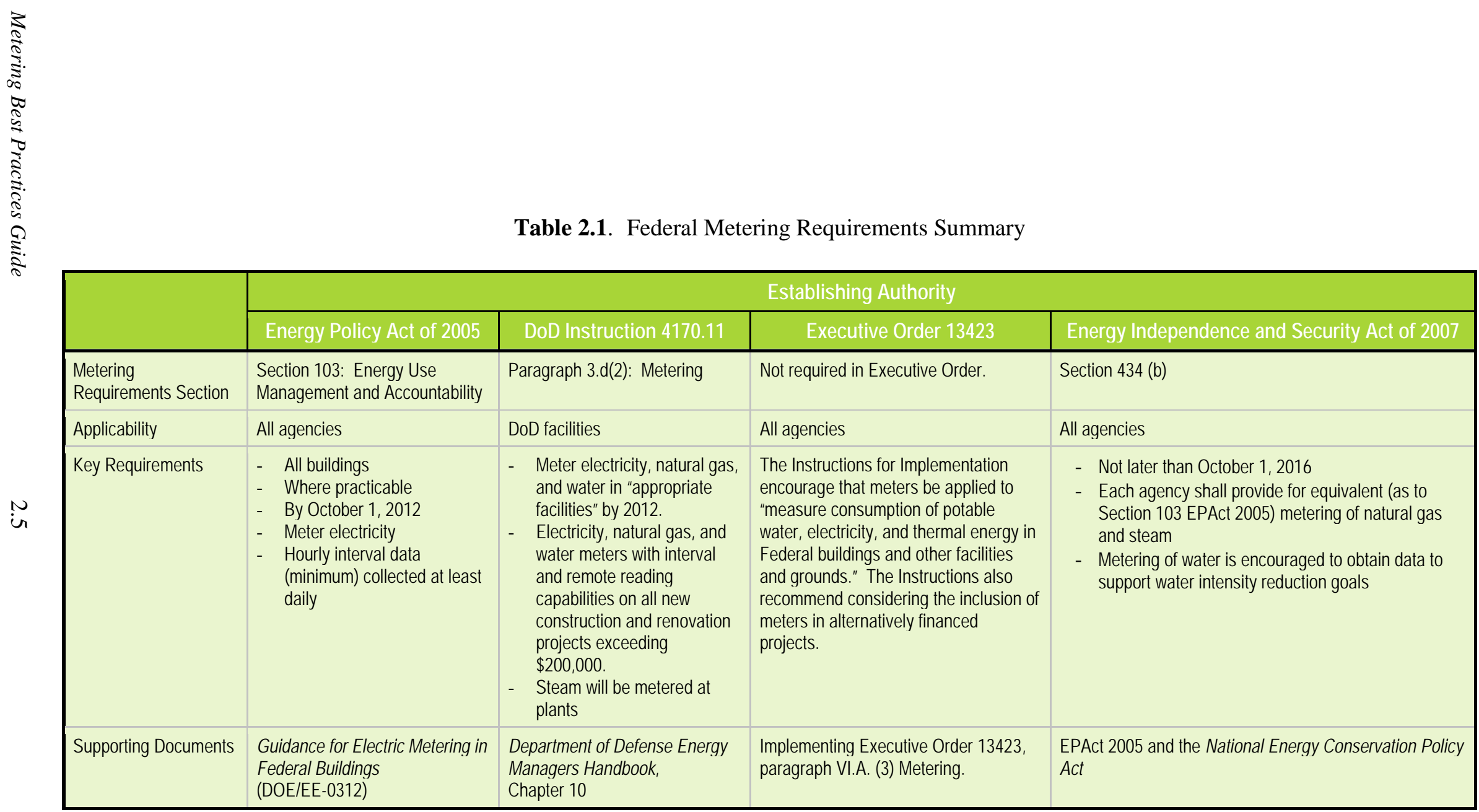


- While there are many potential applications for the smart grid, the near-term relevance to the Federal sector comes in the form of economic pricing of the commodity through "real-time pricing" programs and the potential for demand limiting (also known as demand response) activities. While these programs may not be currently available to all Federal customers - it is widely expected that these (and other) grid-based offerings will become accessible over the next 5 years.

- From the metering perspective, many advanced meters have some form of communication and thus are expected to be able to take advantage of at least early smart grid program offerings (e.g., real-time pricing and demand response). As the grid increases in sophistication, new metering and access technologies will likely need be employed.

- The Leadership in Energy and Environmental Design certification for existing buildings (LEED-EB) allows for up to three energy credits for the application of continuous metering on energy-using systems. LEED-EB also requires metering energy output from onsite renewable energy systems. Regarding the LEED certification for new construction (LEED-NC), metering may be used in support of the energy credit for measurement and verification.

- Section 2 (g) of EO 13514 requires all new construction, major renovations, or repair and alteration of Federal buildings comply with the Guiding Principles for Federal Leadership in High Performance and Sustainable Buildings. This requirement will necessitate the metering of various resources used in those buildings.

- Section 2(d) of EO 13514 requires agencies to reduce potable water intensity of their sites as well as consumption related to industrial, landscaping, and agricultural water use. Installation of meters can assist agencies in providing creditable data to support these water efficiency efforts.

\subsubsection{Business Case for Metering}

Outside of single-building sites, there is limited building or equipment sub-metering within the Federal sector. Single building sites are metered for total use by their servicing utility providers, while multibuilding sites usually rely on a master meter provided by the utilities at the utilities' points of entry to the site. Sites are billed by their utility providers based on the cumulative usage readings obtained from these utility, or revenue,

How the metered data are used is critical to a successful metering meters over the billing period, usually about one month. The application of meters to individual buildings and even energy-intensive equipment provides program. facility managers and operators real-time information on how much energy has been or is being used. This type of information can be used to assist in optimizing building and equipment operations, in utility procurements, in building energy budget planning and tracking, and so on.

It is important to keep in mind that meters are not an energy efficiency/energy conservation technology per se; instead, meters and their supporting systems are devices that provide building owners and operators with data that can be used to:

- Reduce energy/utility use

- Reduce energy/utility costs 
- Improve overall building operations

- Improve equipment operations.

How the metered data are used is critical to a successful metering program. Depending on the type of data collected, these data can enable the following practices and functions:

- Verification of utility bills

- Comparison of utility rates

- Proper allocation of costs or billing of reimbursable tenants

- Demand response or load shedding when purchasing electricity under time-based rates

- Measurement and verification of energy project performance

- Benchmarking building energy use

- Identifying operational efficiency improvement opportunities and retrofit project opportunities

- Usage reporting and tracking in support of establishing and monitoring utility budgets and costs, and in developing annual agency energy reports.

Ultimately, the business case for metering energy/utility use is based on the anticipated benefits to the site. Most of the metered data uses listed above will result in energy cost savings that can be used to justify the cost to purchase, install, and operate the metering system. The degree of cost savings realized depends on the unit cost of the energy/utility being saved and on the effectiveness with which the site analyzes the data and acts upon its

Ultimately, the business case for metering energy/ utility use is based on the anticipated benefits to the site. findings/recommendations. But other potential benefits should also be considered as part of the metering business case. Examples can include:

- Supporting efforts to attain ENERGY STAR and/or LEED-EB certifications

- Promoting tenant satisfaction by providing information that tenants find useful in managing their operations

- Prolonging equipment life (and reducing capital investment requirements) and improving its reliability by verifying the efficient operation of equipment

- Assessing the impact of utility price fluctuations prior to or as they happen, allowing sites/agencies to address budget shortfalls on a proactive basis.

\subsubsection{Preparing for the Future}

We are not clairvoyant about the state of metering in the future. However, it is a given it will occur in response to new material, electronic, and sensor development, as well as new and additional requirements for real-time data information regarding environmental or equipment operating conditions. Future expansion of the metering system should always be considered as well as introduction of new metering and sensor technologies based on the best available information. However, that does not infer all systems 
need to be overdesigned, but all metering systems and sensors will have a finite shelf life as new technologies come into the marketplace and older technologies are considered obsolete and not supported by equipment and software companies.

\subsection{References}

42 USC 8253(e)(1). 2007. “Metering of Energy Use.” National Energy Conservation Policy Act, as amended.

Energy Independence and Security Act of 2007 (EISA 2007). 2007. Public Law 110-140. Signed December 19, 2007.

Energy Policy Act of 2005 (EPAct 2005). 2005. Public Law 109-58, as amended, Section 103, Energy Use Measurement and Accountability, Section 543 (42 USC 8253), (e) Metering of Energy Use.

Executive Order (EO) 13514. 2009. Federal Leadership in Environmental, Energy, and Economic Performance. Signed October 8, 2009.

Executive Order (EO) 13423. 2007. Strengthening Federal Environmental, Energy, and Transportation Management. Signed January 24, 2007.

U.S. Department of Defense (DoD). 2009. Department of Defense Instruction 4170.11, Installation Energy Management. Available at: http://www.dtic.mil/whs/directives/corres/pdf/417011p.pdf.

U.S. Department of Defense (DoD). 2005. Department of Defense Energy Manager's Handbook. Available at: http://www.wbdg.org/ccb/DOD/DOD4/dodemhb.pdf.

U.S. Department of Energy (DOE). 2007. The Smart Grid: How a Smarter Grid Works as an Enabling Engine for our Economy, Our Environment, and Our Future. Prepared for the U.S. Department of Energy by Litos Strategic Communications under contract No. DE-AC26-04NT41817, Subtask 560.01.04.

U.S. Department of Energy (DOE). 2006. Guidance for Electric Metering in Federal Buildings. DOE/EE-312. Available at: http://www1.eere.energy.gov/femp/pdfs/adv_metering.pdf.

\section{Website:}

U.S. Green Building Council (LEED for Existing Buildings): http://www.usgbc.org/DisplayPage.aspx?CMSPageID=221. 


\section{Chapter 3 Metering Planning}

\subsection{Introduction}

EPAct 2005 requires that all Federal buildings be metered for electricity, where practicable, by October 1, 2012. EISA 2007 requires that all Federal buildings be metered for natural gas and steam, where practicable, by October 1, 2016. In addition, while Executive Order 13514 does not require Federal buildings to be metered for water, installation of meters is the best way to establish a credible baseline regarding actual consumption. This allows agencies several years to design and phase in all activities associated with installing their metering systems and rightfully so since implementing a metering program requires thorough planning. In this case, planning refers to a deliberative process that, at a minimum, identifies key actions, milestones, and responsible individuals that will result in a successfully installed and operating metering system and program. In the end, a successful metering system and/or program will satisfy each of these objectives:

- Provide appropriate and accurate data in a timely manner

- Complete the analysis of data in a timely manner

- Provide data and analysis results to users in a format that leads to actions

- Operate continually and effectively on a daily and annual basis.

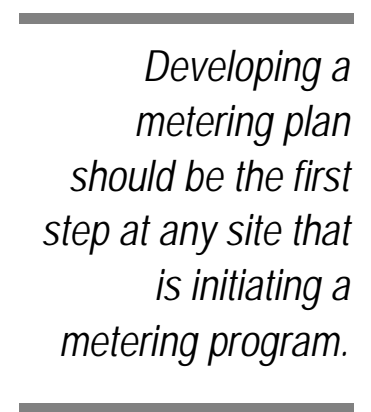

\subsection{The Need for a Metering Plan}

Developing a metering plan should be the first step at any site that is initiating a metering program. A site metering program can take several years to fully implement, especially at sites that have many buildings and/or sites that will meter multiple utilities. Over this implementation period, a number of significant and potentially complex questions will need to be addressed, and sometimes difficult decisions made. Effectively addressing these questions and issues will be greatly assisted by having a metering plan in place that:

- Establishes metering program objectives

- Identifies current and future metering needs for all energy and water utilities

- Identifies recovery procedures for the situation when the metering stops working or deemed out of calibration and data is lost or considered questionable

- Offers the opportunity to obtain and maintain management and stakeholder support

- Ensures consistency in decision making as the program development process moves forward

- Identifies key program milestones and assign individuals with lead responsibilities based on defined competency level

- Details the training and retraining requirements for metering and data analysis

- Provides for ongoing metering program monitoring and, where appropriate, adaptation. 


\subsection{Steps to Consider in Your Metering Plan}

There are many approaches a site can use to develop a metering plan. The approaches used at a given site will vary based on factors such as site mission, building construction, geography, utility expenses, and agency policy. This means a one-size-fits-all approach should not be applied to the development of a site metering plan.

Whichever approach a site uses in its planning effort, there are key elements that should be addressed for all utility metering programs (FEMP 2007):

- Establish program goals and objectives

- Identify needs to support selected data analysis approaches

- Addresses frequency of data collection and reporting

- Develop and apply evaluation criteria

- Implementation, design, and installation expectations

- Performance validation and persistence.

Figure 3.1 provides a flow chart of the key steps in the metering planning process.

\subsubsection{Establish Program Goals, Objectives, and Risk Mitigation}

\section{Some Questions to Consider in Developing Your Metering Objectives}

- What are the annual utility costs for your facility?

- Who are the primary energy users and why?

- What operations actions can help reduce utility costs?

- Where is the poorly designed or operating equipment?

- What equipment should be replaced and when?

- Do like buildings use similar amounts of energy?

- Do buildings have similar operating schedules?

- Do buildings have unique operating requirements?

- By building, how much energy do you use daily? Weekly? Monthly?

- Are your energy savings strategies/projects producing results?

- What utility rate opportunities can you take advantages of?

- Are there regional or national/agency initiatives to address specific utility usage issues (e.g., water management)

- Has utility price volatility been, or could it be, an issue at your site?

- What is the minimum data recovery requirement?
The critical first step for all metering programs is to establish the site's overall metering objective. While the ultimate goal of the metering program is to reduce utility use and/or costs, how this is done will depend on how the metered data are used. Some of the more typical uses include cost allocation among tenants, bill verification, demand side management, and energy use diagnostics. (A complete list of metered data uses is presented in Chapter 7.) Examples of possible objectives might be:

- To fully enable energy bill allocation throughout an entire facility.

- To effectively manage electric loads to minimize costs under a time-based rate schedule.

- To identify system-specific operational efficiency opportunities. 
- To identify equipment malfunction or impending malfunction in critical use facilities, such as hospitals.

- To formalize the outcomes of each objective. For example, if the objective is to enable full bill allocation, an outcome might be to reduce energy costs by 10 percent. If the objective is to minimize costs under a time-based rate schedule, an outcome might be to reduce electric demand charges by 20 percent or eliminate the trigger of additional cost due to excessive demand use.

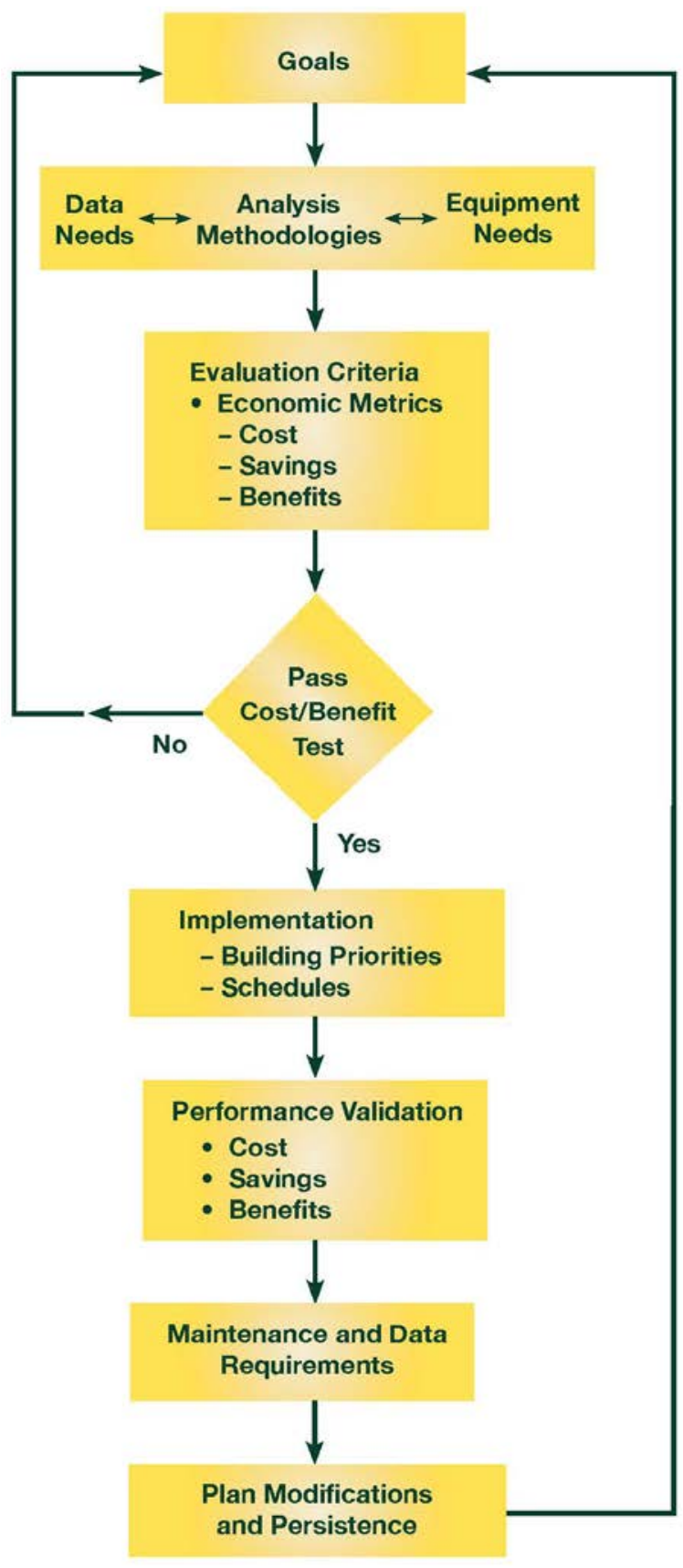

Figure 3.1. Metering Planning Process 


\subsubsection{Identify Needs to Support Selected Analysis Approaches}

The information obtained in this step is used to ensure that the necessary data are obtained and its analysis is supported. Inclusive is the survey of any existing metering components that are operational and in use as they may support the new program's goals and objectives.

\section{Management and Stakeholder Support}

Obtaining the short- and long-term support necessary for a successful metering program begins with having the buy-in of the building or facility manager and its stakeholders occupants or programs that are affected by the availability and cost of the utility services, and the IT staff. Enlisting these parties in the development of your goals and objectives provides an opportunity to educate them on the potential benefits of metering to them, hear their concerns, incorporate their operational requirements into the overall plan, and prepare them to be users of the system when installed.
- Data needs serves as the starting point for this portion of the plan's development. That is, the specific types of data that are needed to support the program's goals and objectives. For example, allocation electricity costs based on actual use will require (at a minimum) $\mathrm{kWh}$ and $\mathrm{kW}$ data at the building level or for portions of the buildings occupied by different tenants. In addition, the metering system will need to have the ability to identify and notify each organizational unit of its consumption and demand on a periodic basis. Table 3.1 presents the types of data required to support various building electricity metering approaches.

- Analysis methodologies are a critical component of a site's metering program. Data by itself is not of much use without some analysis to determine what it means. There are many tried and true methods of trend analysis, for example, and many commercially available software tools and service providers that can help make sense out of the enormous amounts of data. This is especially critical in formulating resource use at the site level for those sites with multiple buildings being metered. Information regarding the site load profile in the various seasons as well as the overall base load will be useful in determining if the site should switch to an alternative rate structure, or participate in available demand-response programs that could reduce the overall resource cost to the site.

- Equipment needs are based on the data requirements and the analysis methodologies identified, and should identify what types of metering/

An important question to ask is what specific types of data are needed to support the program's goals and objectives. monitoring equipment and hardware/software tools would be most appropriate to provide that data and its communication and storage.

- Survey existing metering systems. Many multiple building sites have some level of building metering or sub-metering in place. When this is the case, a survey of this existing metering equipment that identifies the type of meter, its location, operating status, capabilities, and actual data collection/ communications and applications needs to be completed. Can the existing metering system support the new program's goals and objectives? Can the existing equipment be put to better use?

- Staffing resources needed to operate the metering system when in place are also critical to a successful metering program. This includes the staff necessary to start up the metering system as well as the staff/contracted support that will provide the ongoing operations and maintenance of the installed metering system as well as data storage and retrieval. 
- Security requirements vary widely across the Federal sector and are becoming more rigid. In general, information technology (IT) staff should be asked to participate in the development of the metering program planning efforts at the very beginning of the process to determine if data can be transmitted over an existing IT system, or in a separate communication system will be required.

- Starting with the end in mind is a recommended approach in metering planning, particularly when considering anticipated data needs. By considering desired outputs and actionable information at the outset of metering planning, the system development and planning will become more focused.

Sections 7.1 and 7.2 present information on data analysis and uses for data that are worth considering as metering plans are formulated.

Table 3.1. Example Electric Data Requirements (AEC 2003)

\begin{tabular}{|c|c|c|c|}
\hline Goal & Metering Points & Data Interval & $\begin{array}{l}\text { Minimum Update } \\
\text { Rate }\end{array}$ \\
\hline Cost Allocation & $\begin{array}{l}\text { Demand and energy for each } \\
\text { tenant or agency to be billed }\end{array}$ & $\begin{array}{l}\text { As frequent as required to } \\
\text { support utility rate }\end{array}$ & Monthly \\
\hline Load Aggregation & $\begin{array}{l}\text { Demand and energy for each } \\
\text { facility to be included in the } \\
\text { aggregation }\end{array}$ & $\begin{array}{l}\text { As frequent as required to } \\
\text { support utility rate structures }\end{array}$ & Monthly \\
\hline Utility Rate Analysis & Demand and energy & $\begin{array}{l}\text { As frequent as required to } \\
\text { support utility rate structure }\end{array}$ & $\begin{array}{l}\text { Monthly, or as required to } \\
\text { support analysis }\end{array}$ \\
\hline $\begin{array}{l}\text { Power Quality - typically } \\
\text { appropriate to critical } \\
\text { equipment requirements }\end{array}$ & $\begin{array}{l}\text { Suggested: } \\
\text { Amps, volts, VAR, harmonic } \\
\text { data }\end{array}$ & $\begin{array}{l}\text { As frequent as required for } \\
\text { waveform capture }\end{array}$ & Daily \\
\hline Energy System Diagnostics & $\begin{array}{l}\text { Depends on types of } \\
\text { diagnostics, use demand and } \\
\text { energy for consumption- } \\
\text { related diagnostics }\end{array}$ & $\begin{array}{l}\text { Suggestions: } \\
\text { - } 15 \text { minutes } \\
\text { - } \quad \text { Shorter intervals for end- } \\
\text { use diagnostics involving } \\
\text { cycling analysis }\end{array}$ & $\begin{array}{l}\text { Daily } \\
\text { More frequently for real-time } \\
\text { analysis and reporting }\end{array}$ \\
\hline $\begin{array}{l}\text { Monitoring and Verification } \\
(\mathrm{M} \& \mathrm{~V})\end{array}$ & Demand and energy & $\begin{array}{l}\text { As frequent as required to } \\
\text { support M\&V requirements; } \\
\text { hourly may be sufficient }\end{array}$ & Monthly \\
\hline Design Information & Demand and energy & Hourly or daily & $\begin{array}{l}\text { As required for design } \\
\text { projects }\end{array}$ \\
\hline $\begin{array}{l}\text { Management Reporting } \\
\text { Requirements }\end{array}$ & $\begin{array}{l}\text { Depends on reporting } \\
\text { requirements; demand and } \\
\text { energy for consumption- } \\
\text { related reporting }\end{array}$ & $\begin{array}{l}\text { Depends on reporting } \\
\text { requirements }\end{array}$ & $\begin{array}{l}\text { As required for reporting } \\
\text { frequency }\end{array}$ \\
\hline
\end{tabular}

Not included in Table 3.1 are the goals and data needs for two increasingly common system-level activities: onsite load shedding and utility-sponsored demand response programs. In both these programs metering should be provided to accurately quantify energy use at the system-level or at the point of use being affected. The requisite data interval is the utility standard 15-minute with an ability to view/update on an hourly basis.

\subsubsection{Develop and Apply Evaluation Criteria}

Meters should be applied where they will lead to a cost-effective reduction in

Meters should be
applied where they
will lead to a cost-
effective reduction in
utility use and/or
costs.
utility use and/or communication costs. Determining which buildings can be 
metered cost-effectively requires that criteria be established and applied that take into account the lifecycle costs to meter and the benefits to be realized. For example, sites may decide to install meters on all utilities at the building level when the estimated simple payback period is 10 years or less. In some cases, the criteria are stipulated (see DoD Instruction 4170.11, DoD 2009), but in the remaining cases, a determination of cost-effectiveness will need to be made.

The primary variables that impact the cost-effectiveness of meters are (see Section 3.4 for detailed discussion):

- The annual utility cost of the building being metered

- The cost to purchase and install the meter and associated hardware

- Expected savings resulting from the productive use of data, typically in the range of 2 percent to 10 percent, but sometimes higher depending on how the metered data are used

- Site economic criteria - usually payback period.

There are other costs that should be considered in any cost-benefit analysis such as maintenance cost on a per meter basis as well as cost for maintaining the collected data and any analysis results as well as ancillary cost such as meter calibration activities.

In cases where a particular application does not pass the evaluation criteria, the input variables - and possibly the evaluation criteria - should be reviewed and, when appropriate, adjusted to allow for re-evaluation. For example:

- Review estimated building utility consumption estimates. Does the estimated consumption account for energy-intensive operations or building operations during nights or on weekends?

- Does the cost of the meter used in the estimate include functionality that exceeds the stated goals?

- Can the estimated savings be revised? Are there additional uses for data that will result in increased energy and/or cost savings?

- Should the payback period be lengthened?

Cases where applications do not pass the site-developed criteria should be documented. This will allow the potential application to be reconsidered once the program is up and running and as economic (utility rate increases) and policy directions (laws, executive orders, regional initiatives) dictate.

\subsubsection{Implementation, Design, and Installation}

There may be a need or a greater benefit to metering some buildings and/or some utilities before others.
The planning process up to this point has been largely analytical. Based on the goals, objectives, analysis needs, and application of evaluation criteria, there is now enough information to design the actual metering system. Elements of the implementation, design, and installation steps include the following subelements discussed below:

- System financing, or how much funds are available and how will these funds be obtained, needs to be addressed early in the design process and revisited once cost estimates based on actual designs are completed. Section 3.5.2 
provides a summary of the many financing considerations for Federal sites in terms of how a project may be funded and which funding option is best for them.

- Prioritization of buildings and/or utilities to be metered. There may be a need or a greater benefit to metering some buildings and/or some utilities before others. For example, funding will be made available incrementally over several years, while initial applications may be based on total electricity use or geography (buildings in close proximity to each other).

- The design of the metering system hardware application needs to

- Satisfy current functional requirements

- Define a system architecture

- Anticipate future available technology products

- Develop equipment specifications

- Review and refine the cost estimate to purchase and install the metering system.

- An installation plan that addresses the following:

- A timeline for the installation of equipment at buildings and on subsystems. Installation plans at Federal sites should ensure that the EPAct 2005 deadline of October 1, 2012, for metering electricity in buildings is met and existing plans have been modified to incorporate metering of both steam and natural gas use, if present, by the EISA 2007 deadline of October 31, 2016. Agencies should also consider metering of water consumption in order to comply with Executive Order 13514 water intensity (building) and water reduction (industrial, landscaping, and agricultural) reduction goals.

- The order of equipment installed when installation is phased.

- Commissioning the system components to ensure that they are operating as intended before accepting the installation as completed.

- Training of site staff responsible for the maintenance of the system components and the ongoing operations activities including data storage and retrieval.

\subsubsection{Performance Validation and Persistence}

Once the metering system is up and running, the overall focus shifts to making sure:

- Accurate data are obtained and put to timely productive use, and

- The metering system continues to operate effectively and reliably.

The application of the data, or, more correctly, the effective analysis of data and the subsequent actions that follow, will lead to utility use and/or cost savings. These are the very savings that were used to justify the installation of the metering system. But of equal importance is the continued effective operation of the metering system and program. The following activities should be addressed in the metering plan to ensure the sustained operation of the metering system and program.

- Identify the funding resource requirements necessary to maintain the metering system equipment and staff (inclusive of outsourcing), data collection, analysis, and reporting (to system users) activities. Funding will need to be provided on an annual basis as part of the facility’s operating budget. Consider including annual training for system operators. 
- Review on a regular basis the effectiveness of the metering program. Are the program's goals being met? If not, what measures should be taken to improve results?

- Assess new opportunities or needs. Re-evaluate potential applications that did not pass the evaluation criteria, especially when utility rates increase or if metering prices decline. Also consider additional applications such as utilities that were not included in the initial plan for energy-intensive equipment.

\subsubsection{Special Metering Considerations}

Data Centers - The energy use associated with data centers has increased markedly since 2000. That increase will continue in the future due to expanding facility automation and equipment upgrades that add new capabilities that create the need for additional servers and the space to house them. Power density and the effective cooling are fast becoming defining issues for the data center operators and energy managers. Thus, the need to improve energy efficiency can no longer be ignored.

While equipment manufacturers are responding to these concerns with equipment that has greater computing capability with less power input and more efficient cooling systems, the need for additional cooling capacity will still be required. This will require more concerted effort to meter energy consumption and ensure available cooling capability will be available. While some data centers are stand-alone buildings, a majority are distributed through existing buildings to meet individual user needs, others are a major portion of a larger building that serves the entire site. In both these cases, sub-metering of energy use and other environmental parameters is required on a real-time basis to ensure continued operation of the data centers. Section 7.3.7 provides more detailed information regarding how this can be addressed.

Onsite Power Generation - Recent legislation has emphasized the need for onsite generation of power to both reduce the nation's dependence on foreign oil and increase the energy security of Federal sites. In addition, there is a growing interest into the application of smart grid technologies. As a result, there has been an increase in the assessment, design, and implementation of various onsite power generation resources, including, but not limited to, fuel cells, renewable technologies (solar, wind, geothermal, ground source heat pump, and methane gas), and siting of combined heat and power systems. This will require that metering systems be designed or expanded to capture information regarding real-time information on the amount of power being generated as well as environmental factors that impact the technology performance. Section 5.8 provides detailed information that addresses these technologies.

\subsubsection{Staffing and Resource Requirements}

Metering systems and the data they generate are not "set-them and forget-them" devices. To achieve any value from these systems there needs to be well-defined commitments in the areas of data usage and system maintenance. Each of these entails a different skill set and resulting resource requirements; both necessitate a commitment of time and resources for the program to be successful.

Assessing the costs to perform data analysis and system maintenance is difficult and highly dependent on the specifics of the system. The number and type of meters used will impact maintenance requirements, and decisions on how to receive and process data will impact analysis costs. 
Data Analysis. Prior to receiving any metered data it is recommended that a meter data management system be in place. These systems can be site-generated spreadsheet/database tools or commercially available software packages. Whatever the mode, it is important to automate as many of the data access and analysis functions as possible.

Once the metering system is in place, data will accumulate very quickly. A typical Federal site with 50 meters, reporting 15-minute energy data and uploading daily, will result in 4,800 data points per day, 33,600 per week, and over 1.7 million per year.

To estimate resource commitments for data analysis, consider the following example:

- System assumption: Fifty whole-building and end-use meters serving electric, natural gas, and steam loads.

- System analytical outputs: Daily plots of energy use presented as times-series metrics in engineering units in a dashboard environment.

- Daily time series demand, therm, and steam plots

- Alarm sets enabled to highlight out-of-range values

- Exception report comparisons, day-of-the week, month-of-the year

- Drill-down capability to 15-minute reporting

- Resource commitments: System Training (one time)

- Staff training on system design, access, and applications: 1-2 days

- Staff access and system navigation mastery: 1 week

- Daily access for system review and assessment: 2 hours

- Resource Commitment: System Use (weekly for 50-meter system)

- Commitment for data access, review, and assessment: 10 hours/week

Note: Actions resulting from data assessments will require additional time/resources.

System Maintenance. With the advent of solid-state components and meters, routine system maintenance has been reduced - but not eliminated. Maintenance requirements will vary with meter type and should be included in standard preventive maintenance routines (FEMP 2010). Chapter 5 of this guide provides specific recommendations by meter type.

- Resource commitments: System Training (one time)

- Staff training on metering technology and function: 2-4 hours per meter type

- Resource Commitment: Functional inspections (monthly for 50-meter system)

- Monthly commitment for preventive maintenance activities: 5 hours/month

Note: Monthly inspections do not include manufacturer-recommended calibration activities.

\subsection{Metering Economics}

We want to apply meters where it makes sense. This means meters should be applied when their application can be justified on the basis of cost-effectiveness - a measure relating the estimated costs to the estimated savings, such as a simple payback period. 


Meters should be
applied when their
application can be
justified on the
basis of cost-
effectiveness.

Determining the cost-effectiveness requires us to estimate

- the cost to design, purchase, install, maintain, store data, and operate the meter/metering system, and analyze the data output, and

- the resulting energy cost savings.

This chapter examines these cost components and demonstrates how to evaluate the economic practicability of a metering application. This chapter also looks at the various financing options available to Federal sites to purchase, install, and potentially operate their metering systems.

\subsubsection{Metering Costs (DOE 2006)}

Metering system costs vary widely for a number of reasons: equipment specifications and capabilities, existing infrastructure, site-specific design conditions, local cost factors, etc. For this reason, this guide does not present specific cost estimates. Instead, we identify the main cost components that should be addressed when developing a metering cost estimate.

\section{How Much Do Meters Really Cost?}

Meter costs cover a range and are affected by a number of variables - type of metering, functionality and accuracy of meters, communications and storage requirements, unique applications and/or installation requirements. Coupled with the rapid evolution of metering products available in the marketplace, it is not practical to provide cost estimates in this guide; instead, system planners and designers are encouraged to contact metering equipment vendors, as well as network with other system planners and designers to tap into their experiences.

Of particular interest to the Federal sector is the cost of an advanced electric metering system and the on-going cost to maintain it. Actual cost information is just now becoming available. Initial reported information indicated the range of the cost of a meter, associated communication equipment, required software, and installation activities would be between $\$ 1,900$ to $\$ 5,400$. On-going cost for a single meter would range from $\$ 10$ per month to almost $\$ 50$ per month (Heller 2005). More recent information regarding installation of electrical meters at Federal sites indicates an average cost of $\$ 2,000$ per meter (see case study information in Section 8.7). Other information indicates a range of $\$ 1,500$ to $\$ 2,500$ based on installed metering systems (Lewis 2010).
The metering cost estimate can be separated into three main categories: capital, labor, and recurring costs. More detailed descriptions of these categories and the types of costs to be included are provided below.

- Capital: Capital refers to the cost of the meters and all materials required to support their installation:

- Meter purchase cost. The purchase price depends on the features selected such as accuracy, memory, and mounting.

- Ancillary devices. Electric meters require current transformers (CTs) and safety switches. These devices may be built-in into a meter but are usually purchased separately.

- Communications module. There are a number of types of communication modules that can be purchased for electric meters: Handheld reader communicator, telephone modem, radio transceiver, power line carrier modem, Ethernet modem, and supervisory control and data acquisition (SCADA) interface RS232RS485. Communications modules are usually ordered with the meter. 
- Miscellaneous supplies. Small compared to other hardware line-item costs, miscellaneous supplies include items such as wire, conduit, and junction boxes necessary to complete the installation.

- Labor: Labor covers the time charges for a crew and should account for planning and prep time, crew travel time, installation of all hardware required for a working installation, connection of the communications module, operational testing, and inspection. Examples of variables in the labor costs include the type of meter being installed (utility being metered and if the meter is intrusive or nonintrusive), service shut-downs that may need to be accomplished during off-hours, and trenching requirements for running cable.

- Recurring costs: Recurring costs are planned regular costs that support the ongoing operation of the meter/metering system.

- Monthly communications fees. These fees will vary based on the communications method selected.

- Data collection and storage.

- Data analysis: Data need to be analyzed on a regular basis (daily and/or weekly) with findings and recommendations issued.

- Operation and maintenance. Meters require periodic calibration and testing.

\subsection{Metering Savings Potential}

The lack of data from metering applications in the Federal sector makes it difficult to estimate the energy cost savings that can be expected from a site-wide metering program. Estimates of energy savings have ranged from 1 to 20 percent. Table 3.2 presents metering-related savings ranges based on different uses for metered data.

As Table 3.2 demonstrates, the savings realized by a metering program depend largely on the actions taken with the data.

The benefits of installed meters are minimal if the meters are simply installed across a site but there is not any follow-up action taken with the data. There may be some initial savings realized due to the knowledge that metering is on-going. However, these savings will rapidly decline over time if the occupants realize that there is no feedback regarding consumption so the data are not being used.

Table 3.2. Metering Savings Ranges (DOE 2006)

\begin{tabular}{|l|l|}
\hline \multicolumn{1}{|c|}{ Action } & \multicolumn{1}{c|}{ Observed Savings } \\
\hline Installation of meters & 0 to $2 \%$ - initial impact, but savings will not persist \\
\hline Bill allocation only & $2 \frac{1}{2}$ to $5 \%$ - improved occupant awareness \\
$\begin{array}{l}\text { Building tune-up and load } \\
\text { management }\end{array}$ & $\begin{array}{l}5 \text { to } 15 \% \text { - improved awareness, identification of simple } \\
\text { operations and maintenance improvements, and } \\
\text { managing demand loads per electric rate schedules }\end{array}$ \\
\hline Ongoing commissioning & $\begin{array}{l}15 \text { to } 45 \% \text { - improved awareness, ongoing identification } \\
\text { of simple operations and maintenance improvements, } \\
\text { and continuing management attention }\end{array}$ \\
\hline
\end{tabular}


The realized savings will increase as the data are more widely applied and there is an improved awareness of consumption. It is anticipated that many Federal sites will implement a metered data cost allocation approach once their metering systems are in place. This, in turn, will lead to increased utility savings since the building occupants will then have a financial incentive to reduce or manage use.

Even greater savings can be realized when the data are used to support actions to optimize building operations. This includes

- Verifying or re-tuning building and/or equipment startup and shutdown times.

- Actively managing electric demand to minimize the impacts of time-based demand charges.

- Determining existing operating conditions for use in building commissioning studies.

- Continued verification of implemented building commissioning activities.

Only in extreme cases should savings greater than 15 percent be considered for estimating benefits. Such a case would be a building or buildings that have a lot of efficiency opportunities due to very poor operations or neglect, a workable project identification and implementation plan exists, and strong site management support.

Validation of the information in Table 3.2 is limited since most Federal agencies are in the process of implementing their electric metering program and do not yet have adequate data to determine whether savings are either at the building or site level. The information that is available is generally limited to short-duration information for specific electrical end use within a building (see Section 8.7), portions of buildings (see Section 8.5), and water consumption at the site level (see Section 8.6). A savings of 43 percent at the site level has been reported for on-going commissioning projects implemented in the commercial sector based on metered data (Lewis 2010).

\subsubsection{Cost Justification Methodology}

\subsubsection{Electric Metering}

EPAct 2005 requires that Federal buildings be metered for electricity "where practicable.” The following formula to cost-justify an electric meter (or other utility meters) was presented in DOE (2006):

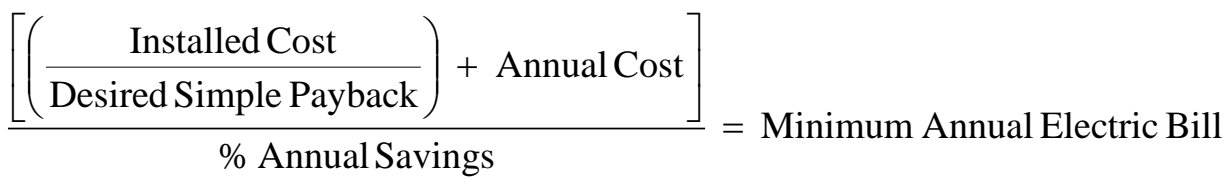

where:

Installed Cost refers

to the total cost to

purchase, install, and

commission the

meter. 
- Annual Cost is the total annual cost of the fees and expenses to cover communications, data collection and storage, and data analysis, as well as meter operations and maintenance. The annual cost will vary based on several factors and is typically in the range of $\$ 120 /$ year $(\$ 10 /$ month) to $\$ 600 /$ year (\$50/month).

- \% Annual Savings is the estimated cost savings benefits to be realized from the productive use of the metered data. Federal sites are advised to use a minimum 2 percent annual savings when considering meters for EPAct 2005 compliance.

Using the above formula also requires that there be a reasonable way to estimate the current annual electricity (utility) costs for the building being considered. Except in cases where the buildings already have standard meters, actual usage data to estimate the annual costs will not be available. In these cases, one of the following accepted methods of estimating building energy use should be applied:

- Square footage - This method estimates energy use by first dividing the total site use by the total site square footage, and multiplying the quotient by the building square footage. This results in a cut-off point for size of buildings for which meters will be applied. Benefits: This approach is quick and simple. Challenges: The approach is not very accurate as decisions do not take into account the uses of energy within the individual building. For example, a small energy-intensive building used for research and development or food service might not be metered while a large, unconditioned, lowenergy-consumption warehouse would.

- Energy-use intensity - The EUI method involves estimating a kWh per square foot per year $\left(\mathrm{kWh} / \mathrm{ft}^{2} / \mathrm{yr}\right)$ value for each building being evaluated. EUIs will vary (primarily) by the building use and climate zone. Benefits: When good data are available, this method can be quickly used with relatively accurate results. Challenges: Obtaining site-specific EUI data. Sources of EUI data include:

- Agency-specific data as collected for internal or reporting purposes

There are four accepted methods of estimating building energy use for cost-justifying meter applications.

- Commercial Buildings Energy Consumption Survey (CBECS) data at http://www.eia.gov/emeu/cbecs

- DOE Buildings Energy Data Book at http://buildingsdatabook.eren.doe.gov

- Calibrated software - This method involves running software simulations to calculate building energy use. Accepted building simulation software includes EnergyPlus and DOE-2. Sites interested in using a multi-building software analysis approach can use the Facility Energy Decision System (FEDS) to complete a site-wide analysis for energy efficiency opportunities and metering practicability. Benefits: Calibrated software can provide accurate results, and models/files may be used to support retrofit project identification. Challenges: Populating the software with site data.

- Short-term metering - This method relies on making use of metering equipment to take short-term meter readings to estimate annual building energy use. Benefits: When done properly, this approach can produce very accurate estimates. Challenges: This approach requires metering equipment, and time to collect and analyze the data. 
To demonstrate how the metering cost justification formula is used, the following values will be used:

- Installed cost $=\$ 5,000$

- Desired simple payback $=10$ years

- Annual cost $=\$ 25 /$ month $=\$ 300 /$ year

- $\%$ annual savings $=2$ percent

Sample calculation: (NOTE: The values in this sample are for demonstration purposes only.) Sites considering metering applications should use values specific to their site.

$$
\frac{\left[\left(\frac{\text { Installed Cost }}{\text { Desired Simple Payback }}\right)+\text { Annual Cost }\right]}{\% \text { Annual Savings }}=\text { Minimum Annual Electric Bill }
$$

$=[(\$ 5,000) \div(10$ years $)+\$ 300 /$ year $] \div(0.02)$

$=\$ 40,000$ minimum annual electric bill

In this example, an electric meter application will be cost-justified if the building's annual electricity use is more than $\$ 40,000$.

As previously mentioned, the results from this equation are sensitive to the input variables. Figure 3.2 demonstrates how the minimum annual electric bill for cost-justified applications becomes smaller as the annual savings percentages increase, while the installed and annual costs and the simple payback period are held constant. Likewise, similar curves can be developed for varying other variables such as the cost of the meter, or changing combinations of variables such as annual percent savings and the cost of the meter.

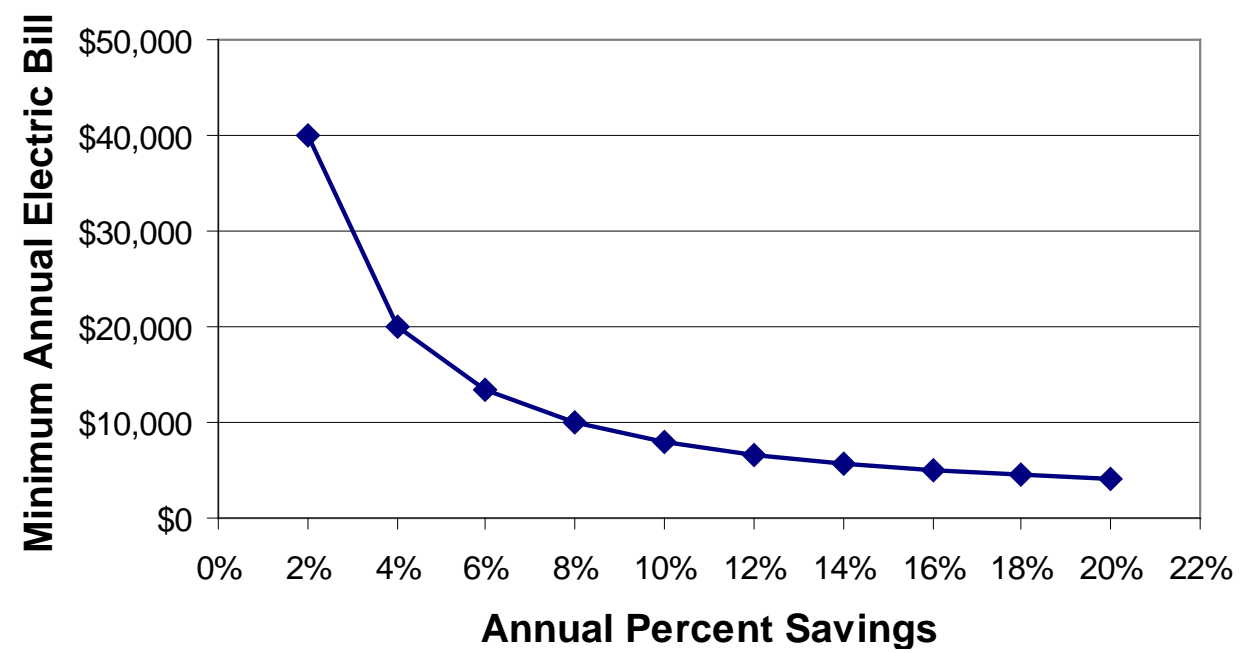

Figure 3.2. Minimum Electric Bill versus Annual Percentage Savings ( $\$ 5,000$ installed cost) 


\subsubsection{Natural Gas Metering}

The Energy Independence and Security Act of 2007 (EISA 2007) extended that metering requirement to nature gas consumption in Federal buildings. Metered data can provide valuable insights into energy consumption in the buildings and/or major equipment that are metered and facilitate more effective operation of the building/equipment. However, there are a large number of Federal buildings, about 430,000 total (FRPR 2010); thus, the real question is how to determine what is a reasonable or "costeffective” when installing an advanced meter?

Typically, natural gas is purchased from a local utility provider and the total gas consumption into the site or complex is measured and recorded on the utility bills. Based on this total consumption, the energy manager of a facility has a method of allocating the gas being used at their site to each building or area; it may be apportioned by square footage, based on one time spot measurements, design of equipment, and/or a software generated model. Regardless of the method, it is assumed the allocated energy consumption data, which will be referred to as "gas consumption" from here on, is a reasonable basis for calculating the annual savings or benefits of installing an advanced meter at a specified location. However, since gas consumption is a critical factor in this analysis, if the estimate is poor or of unknown quality, the energy manager should re-evaluate to verify that the estimate is accurate and improve the estimate, as necessary.

Step One: Determine gas unit cost $\left(\mathrm{C}_{\mathrm{g}}\right)$

The cost of natural gas is needed to calculate the savings. Typically, natural gas is purchased from a local utility provider; thus the unit cost and total consumption are measured and recorded on the utility bills. The following gas prices (Table 3.3) represent the current range of prices across the United States according to the U.S. Energy Information Administration (EIA); ${ }^{2}$ thus, these costs will be used as the basis for unit costs in the calculations.

Table 3.3. Current Range of Natural Gas Prices

\begin{tabular}{|c|c|}
\hline \$cubic feet (\$/ci) & \$/Thous cubic feet (\$/Mci) \\
\hline$\$ 0.005$ & $\$ 5$ \\
$\$ 0.010$ & $\$ 10$ \\
$\$ 0.015$ & $\$ 15$ \\
$\$ 0.020$ & $\$ 20$ \\
\hline
\end{tabular}

Step Two: Estimate building energy consumption and annual savings (S)

The natural gas consumption is needed to calculate the savings associated with adding a meter to an area, building or large equipment. As mentioned, there are various methods of allocation which can vary widely in accuracy depending on how it is derived, the level of effort applied, and the amount and quality of information used. One simple method to enhance or verify an estimate is to base it on the design of the gas-consuming equipment or combination of gas-consuming equipment.

\footnotetext{
${ }^{2}$ March 2010, from EIA website; http://www.eia.gov/dnav/ng/ng_pri_sum_a_epg0_pcs_dmcf_a.htm.
} 


\section{Example based on the design of equipment:}

- A field evaluation compiling information (e.g., nameplate data, vendor documentation, log books, operating schedules) was performed to evaluate a proposal to install a gas meter on a large gas line. The largest gas consuming equipment on the line is a boiler that had a nameplate that described the type of fuel the boiler is designed to burn and the maximum input rate in Btu/hr (Figure 3.3). Note, if the nameplate is difficult to read, or missing, this type of data can often be obtained by the equipment documentation or a call to the equipment manufacturer. In addition, the evaluation can be determined from the operation log book indicating that the boiler operates at 75 percent of the maximum firing rate, 6 months per year. Thus, the gas consumption would be calculated as:

$$
12,247,000 * 75 \text { percent }=9,185,250 \mathrm{Btu} / \mathrm{hr}
$$

annually this would be

$$
=9,185,250 \mathrm{Btu} / \mathrm{hr} *(4,380 \mathrm{hr} / \mathrm{yr} * 1 \mathrm{Mcf} / 1,000,000 \mathrm{Btu})=40,230 \mathrm{Mcf} / \mathrm{yr}
$$

If there was a gas heater (or other gas-consuming equipment) on the same gas line, they could be estimated in the same manner then added to the boiler gas consumption to obtain a total for the line.

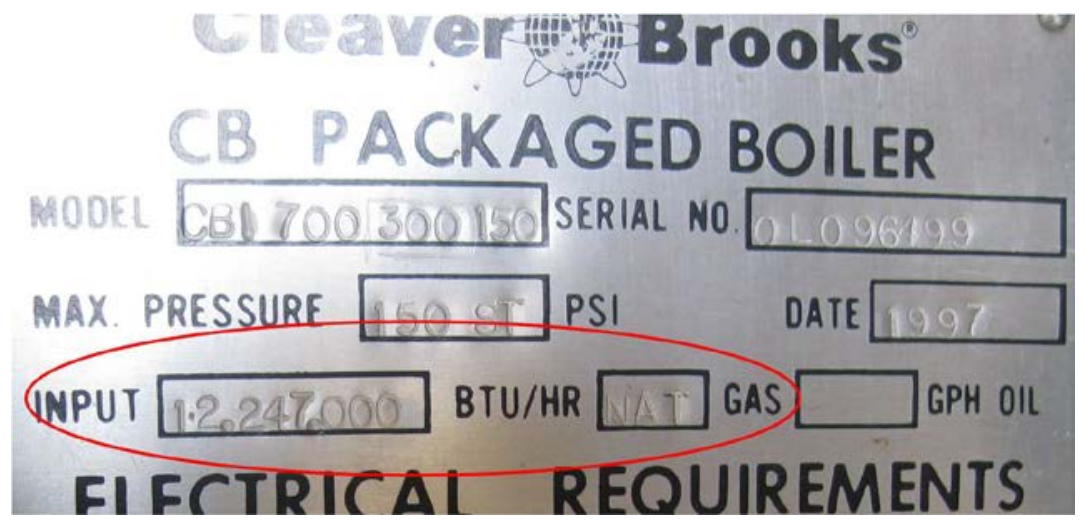

Figure 3.3. Equipment Nameplate

Step Three: Calculate annual savings (S) or use Figure 3.4

Once the cost of gas, the annual gas consumption and expected savings percentage are determined, the annual savings can be manually calculated or obtained from Figure 3.4.

- Using the example in step two, the annual gas consumption =40,230 thousand cubic feet (Mcf) of gas per year

- Gas unit cost $\left(\mathrm{C}_{\mathrm{g}}\right)=\$ 10 / \mathrm{Mcf}$

- The expected savings $\sim 1$ percent on your investment, then...

- Expected annual savings $=40,230 \mathrm{Mcf} * \$ 10 / \mathrm{Mcf} * 1$ percent $=\$ 4,023$ 


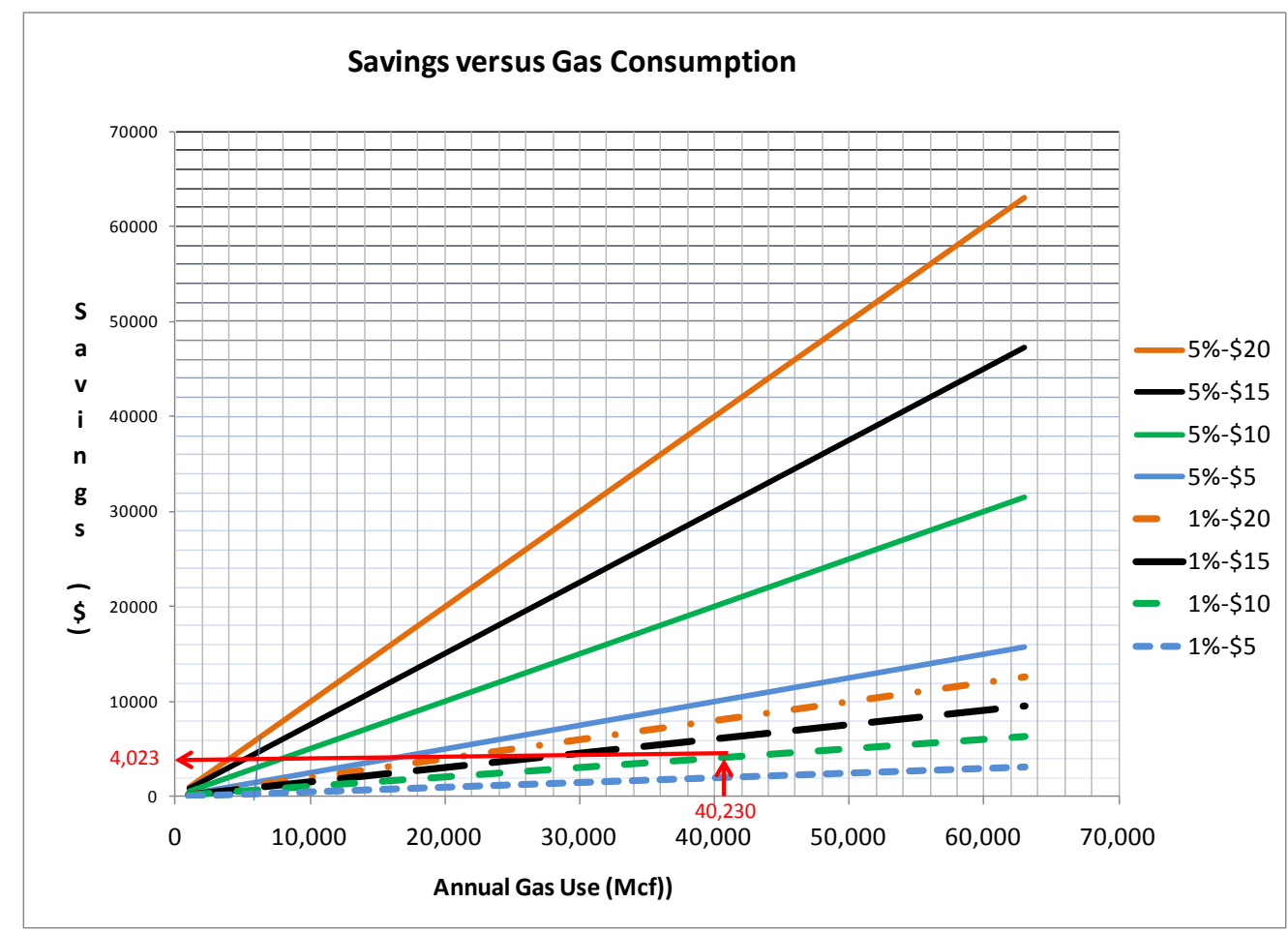

Figure 3.4. Savings Graph versus Gas Consumption @ 1 Percent and 5 Percent Savings

Note: Figure 3.4 provides a range from 1 percent to 5 percent of expected savings, depicting that savings increases as the gas use and cost of gas increases.

Step four includes three cases with the different installed costs of $\$ 2,000, \$ 4,000$, and $\$ 6,000$ to display how these costs impact the savings to investment ratio. Each site will be unique; thus, it is not feasible to attempt to illustrate every condition, but the methods described can be applied to each unique case.

Step Four - Case 1. Calculate savings to investment ratio (SIR) or use Figure 3.5

Once the annual savings, investment costs, and annual operating costs are estimated, the SIR can be manually calculated or obtained from Figure 3.5.

Case 1 - Savings to investment ratio (SIR) calculation using investment costs (IC) $=\$ 2,000$;

Annual savings $(\mathrm{S})=\$ 4,023$, and annual operating costs $(\mathrm{AOC})=\$ 500:\{\mathrm{PV}(\mathrm{S})$ - PV (AOC) $\} / \mathrm{IC}$ :

- Annual savings (S) are calculated above, and converted to a present value (PV) using:

- a 3 percent discount rate (i) per the National Institute of Standards and Technology (NIST) lifecycle costing analysis, and

- conservative expected life (n) of 10 years. The life of a gas meter system (device and transmitter) is expected to be dependent on the application, with some systems in mild conditions (ambient temperatures, clean gas, etc.) having a mean time before failure over 30 years, while some in harsh environments failing in less than a year. 


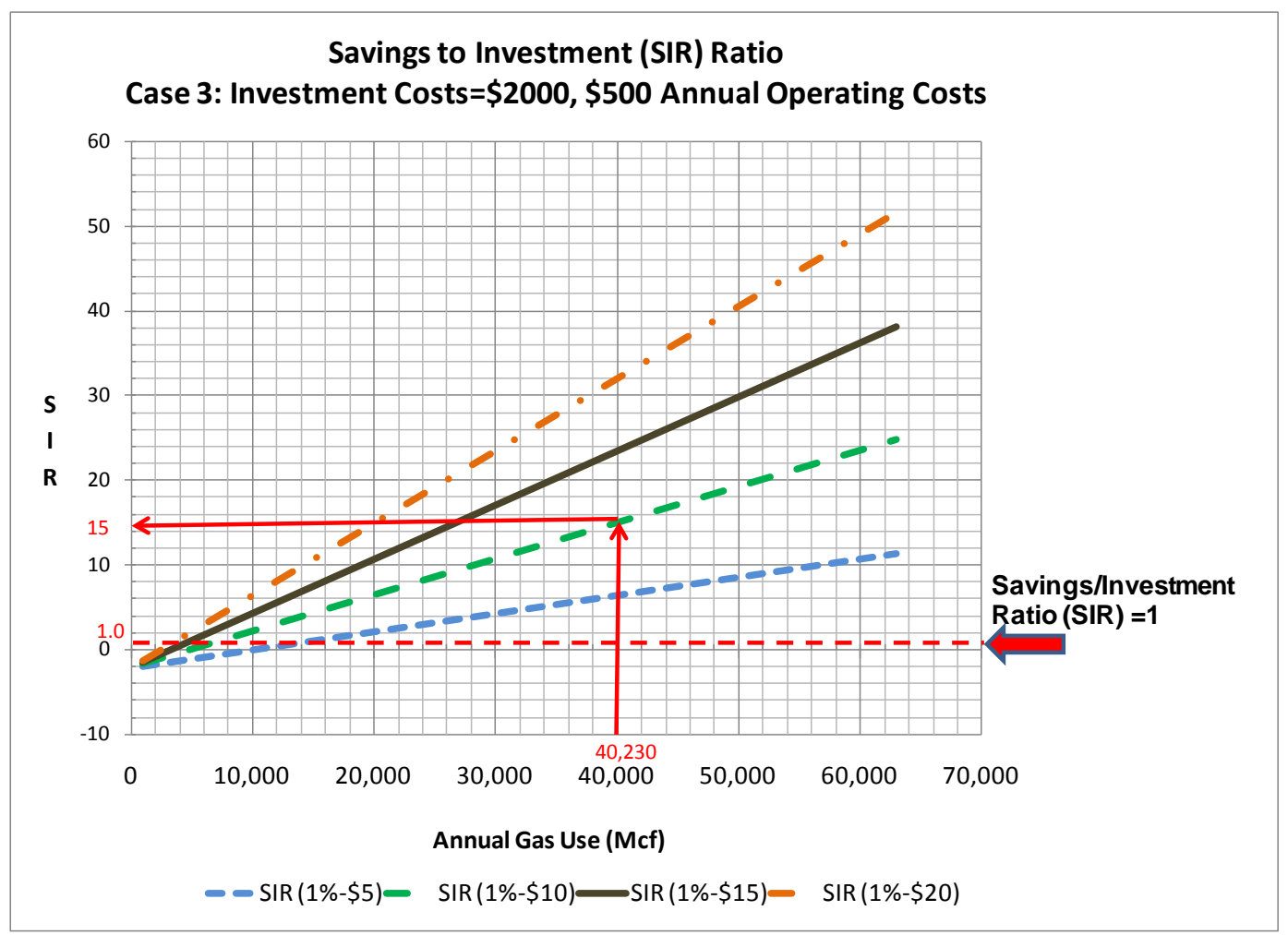

Figure 3.5. Case 1 - Gas Use of 40,000 Mcf versus SIR @ 1 Percent Savings and \$2,000 Installed Costs

- Annual operating costs (AOC) are estimated at \$500/year; representing the costs to maintain and manage the metered data (see Sections 5.3.4 and 8.2). These costs are also converted to PV using the same 3 percent discount rate and 10-year life

- At 3 percent rate (i) and 10-year life (n); P/A factor $=\left(\left(\left(1+0.03^{10}\right)-1\right) /\left(0.03 *(1+0.03)^{10}\right)=\right.$ 8.5302

- The PV of annual savings (S) using P/A factor $=8.5302 * \$ 4,023=\$ 34,317$

- The PV of annual operating costs (AOC) using P/A factor $=8.5302 * \$ 500=-\$ 4,265$

- The PV of the net annual savings $=$ PV( annual savings $)-P V($ annual operating costs $)=$ $\$ 34,317-\$ 4,265=\$ 30,052$

- The SIR ratio $=\$ 30,052 / \$ 2,000=15$.

Since the SIR ratio is $>1$; the benefits outweigh the costs, and is a reasonable project.

Step Four - Case 2. Calculate savings to investment ratio (SIR) or use Figure 3.6. Once the annual savings are determined, investment costs, annual operating costs are estimated; the SIR can be manually calculated or obtained from Figure 3.6. 


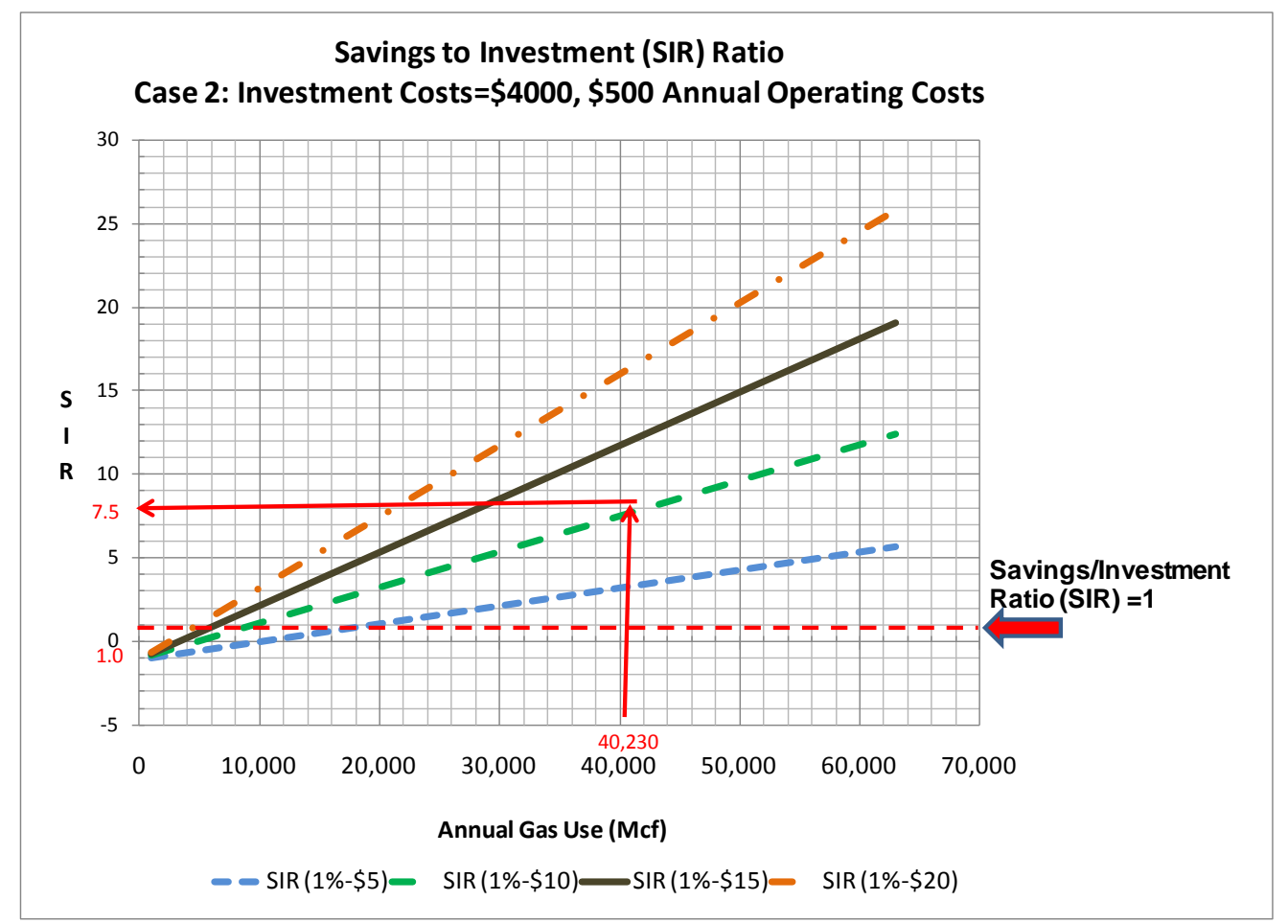

Figure 3.6. Case 2 - Gas Use of 40,000 Mcf versus SIR @ 1 Percent Savings and $\$ 4,000$ Installed Costs

Case 2 - Savings to investment ratio (SIR) calculation using investment costs (IC) $=\$ 4,000$;

Annual savings $(\mathrm{S})=\$ 4,023$, and annual operating costs $(\mathrm{AOC})=\$ 500:\{\mathrm{PV}(\mathrm{S})-\mathrm{PV}(\mathrm{AOC})\} / \mathrm{IC}$ :

Example calculation of (SIR) ratio using the assumptions from above and calculating:

- At 3 percent rate (i) and 10-year life (n); P/A factor $=\left(\left((1+0.03)^{10}\right)-1\right) /\left(0.03 *(1+0.03)^{10}\right)=$ 8.5302

- The PV of annual savings (S) using P/A factor $=8.5302 * \$ 4,023=\$ 34,317$

- The PV of annual operating costs (AOC) using P/A factor $=8.5302 * \$ 500=-\$ 4,265$

- The PV of the net annual savings $=\mathrm{PV}$ ( annual savings $)-\mathrm{PV}($ annual operating costs $)=$ $\$ 34,317-\$ 4,265=\$ 30,052$

- The SIR ratio $=\$ 30,052 / \$ 4,000=7.5$

Since the SIR ratio is $>1$; the benefits outweigh the costs, and is a reasonable project.

Step Four - Case 3. Calculate savings to investment ratio (SIR) or use Figure 3.7. Once the annual savings are determined, investment costs, annual operating costs are estimated; the SIR can be manually calculated or obtained from Figure 3.7. 


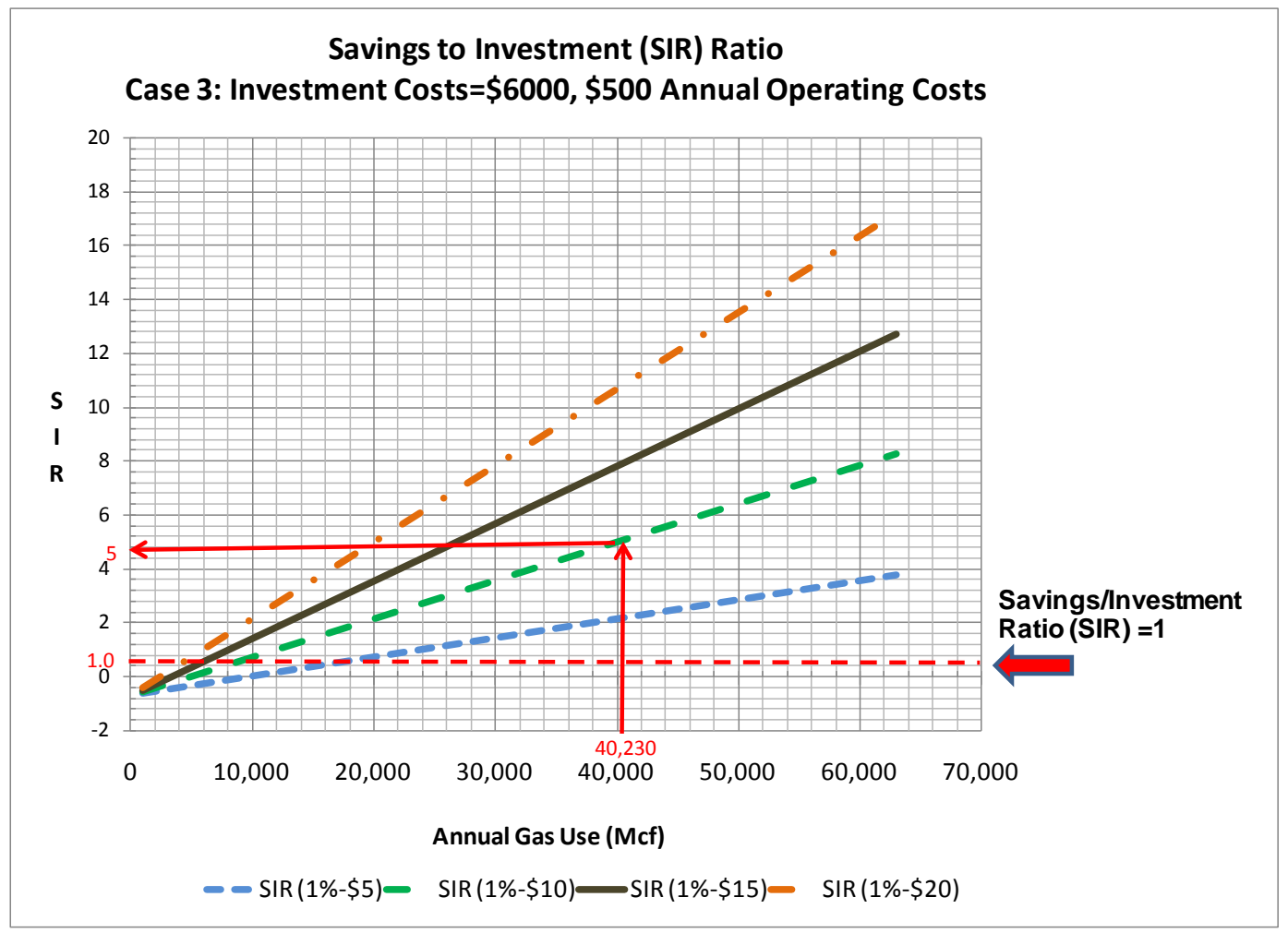

Figure 3.7. Case 3 - Gas Use of 40,000 Mcf versus SIR @ 1 Percent Savings and \$6,000 Installed Costs

Case 3 - Savings to investment ratio (SIR) calculation using investment costs (IC) $=\$ 6,000$;

Annual savings $(\mathrm{S})=\$ 4,023$, and annual operating costs $(\mathrm{AOC})=\$ 0.5 \mathrm{k}:\{\mathrm{PV}(\mathrm{S})-\mathrm{PV}(\mathrm{AOC})\} / \mathrm{IC}$ :

Example calculation of (SIR) ratio using the assumptions from above and calculating:

- At 3 percent rate (i) and 10-year life $(n)$; P/A factor $=\left(\left((1+0.03)^{10}\right)-1\right) /(0.03 *(1+0.03))=8.5302$

- The PV of annual savings (S) using P/A factor $=8.5302 * \$ 4,023=\$ 34,317$

- The PV of annual operating costs (AOC) using P/A factor $=8.5302 * \$ 500=-\$ 4,265$

- The PV of the net annual savings $=\mathrm{PV}($ annual savings $)-\mathrm{PV}($ annual operating costs $)=$ $\$ 34,317-\$ 4,265=\$ 30,052$

- The SIR ratio $=\$ 30,052 / \$ 6,000=5.0$.

Therefore, the energy manager simply needs to identify which set of assumptions most represents their situation to determine whether the meter installation project is cost effective or the savings outweigh the costs.

\subsubsection{Steam Metering}

The Energy Independence and Security Act of 2007 (EISA 2007) extended the metering requirement to steam consumption in Federal buildings. Metered data can provide valuable insights into energy 
consumption in the buildings and/or major equipment that are metered and facilitate more effective operation of the building/equipment. However, there are a large number of Federal buildings, about 430,000 total; thus, the question is how to determine what is a reasonable or "cost-effective" when considering installing an advanced meter in a building that uses steam? Steam can be purchased or generated onsite. If it is purchased, the total consumption and unit cost are typically measured and recorded on the utility bills; however, if steam is generated onsite, then the unit cost and the consumption need to be determined.

Every energy manager of a facility has a method of allocating the energy consumption being used at their site to each building or area; it may be apportioned by square footage, or based on short-term measurements (further discussion in Section 3.4.1). Regardless of the method, it is assumed the allocated energy consumption data, which will be referred to as "steam consumption" from here on, is the best basis for calculating the annual savings or benefits of installing an advanced meter at a specified location. However, since steam consumption is a critical factor in this analysis, if the estimate is poor or of unknown quality, the energy manager should re-evaluate to verify that the estimate is accurate and improve the estimate, as necessary. The quantity of steam being generated can be estimated based on two methods: the design of the equipment (i.e., the boiler output capacity, load, and runtime hours) and/or based on the design flow rates, steam pressure, and pipe sizes.

Step One - Determine steam cost $\left(\mathrm{C}_{\mathrm{s}}\right)$

The cost to generate steam is needed to calculate the savings. The variable costs that are typically associated with generating steam include:

- Fuel, $\mathrm{C}_{\mathrm{F}}$

- Boiler feed water treatment costs, $\mathrm{C}_{\mathrm{W}}$

- Feed water pumping costs, $\mathrm{C}_{\mathrm{P}}$

- Combustion air fan (induced draft and/or forced draft) costs, $\mathrm{C}_{\mathrm{A}}$

- Environmental emissions control/fees, $\mathrm{C}_{\mathrm{E}}$

- Maintenance materials and labor (may be deemed a fixed cost if not related to boiler runtime), $\mathrm{C}_{\mathrm{M}}$

The summation of all these costs (and any additional costs specific to a particular site) will define the cost of generating steam. This cost is typically expressed in dollars per thousand pounds of steam $(\$ / \mathrm{klb})$. The expression given as:

$$
\mathrm{C}_{\mathrm{S}}=\mathrm{C}_{\mathrm{F}}+\mathrm{C}_{\mathrm{W}}+\mathrm{C}_{\mathrm{P}}+\mathrm{C}_{\mathrm{A}}+\mathrm{C}_{\mathrm{E}}+\mathrm{C}_{\mathrm{M}}
$$

Although all these costs contribute to the total cost, typically the cost of fuel $\left(\mathrm{C}_{\mathrm{F}}\right)$ is the overriding cost. However, fuels, such as coal or \#6 oil, can generate significant costs associated with handling, disposal, maintenance, environmental emissions; therefore, a calculation based on the specific infrastructure at a given site should be done to accurately compute the cost of steam. Pending a calculation, 0.30 is 
considered a reasonable estimate to approximate these costs $\left(C_{W}+C_{P}+C_{A}+C_{E}+C_{M}\right)$, associated with steam generation. ${ }^{3}$ Thus, the expression can be written as:

$\mathrm{C}_{\mathrm{S}}=\mathrm{C}_{\mathrm{F}}+\left(\mathrm{C}_{\mathrm{W}}+\mathrm{C}_{\mathrm{P}}+\mathrm{C}_{\mathrm{A}}+\mathrm{C}_{\mathrm{E}}+\mathrm{C}_{\mathrm{M}}\right)$, or

$\mathrm{C}_{\mathrm{S}}=\mathrm{C}_{\mathrm{F}}(1+0.30)$

The cost of fuel can be defined as $\mathrm{C}_{\mathrm{F}}(\$ / \mathrm{klb})=\mathrm{A}_{\mathrm{F}} *\left(\mathrm{H}_{\mathrm{s}}-\mathrm{H}_{\mathrm{w}}\right) / 1,000 / \pi_{\mathrm{B}}$,

where $\quad A_{F}=$ utility billed cost for natural gas, oil, coal, etc. ( $\left.\$ / \mathrm{mmBtu}\right)$.

$\mathrm{H}_{\mathrm{s}}=$ enthalpy of saturated steam from steam tables at the operating pressure, (Btu/lb).

$\mathrm{H}_{\mathrm{w}}=$ enthalpy of feed water from steam tables at the feed water temperature, (Btu/lb).

$\pi_{\mathrm{B}}=$ boiler combustion efficiency.

For example, a project is being considered to add a meter to a 15-psig saturated steam header off a 100 -HP boiler that is burning natural gas at a unit cost of $\$ 5.6 / \mathrm{mmBtu}$. The boiler operates at 80 percent efficiency and receives feed water at an average temperature of $100^{\circ} \mathrm{F}$, then the cost of steam can be calculated using the expression $\mathrm{C}_{\mathrm{S}}=\left\{\mathrm{C}_{\mathrm{F}} *(1+0.3)\right\}$, and $\mathrm{C}_{\mathrm{F}}=\mathrm{A}_{\mathrm{F}} *\left(\mathrm{H}_{\mathrm{s}}-\mathrm{H}_{\mathrm{w}}\right) / 1,000 / \pi_{\mathrm{B}}$.

- Based on the steam tables, the enthalpy of steam, $\mathrm{H}_{\mathrm{s}}$ (@15 psig 30 psia)=1,164 Btu/lbm, and the enthalpy of boiler feed water $\mathrm{H}_{\mathrm{w}}\left(@ 100^{\circ} \mathrm{F}\right)=68 \mathrm{Btu} / \mathrm{lbm}$.

- Based on the given cost of natural gas and the operating efficiency data:

$\left.-\mathrm{C}_{\mathrm{F}}=\left\{\left(\left(5.6 / 10^{6}\right) *(1,164-68)\right) * 1,000\right)\right\} / 0.8=\$ 7.7 / \mathrm{klb}$.

$-\mathrm{C}_{\mathrm{S}}=7.7 * 1.3=\$ 10 / \mathrm{klb}$; the equation results in the energy required to produce 1,000 pounds of saturated steam.

Step Two - Estimate steam generated and annual savings (S)

Method 1 - Based on the design of equipment using the example in Step One:

- The boiler log for the 100-HP boiler shows that it operates at approximately 80 percent of full rating, 24 hrs per day for 6 months per year to be $\left(34.5^{4} * 100 \mathrm{HP} * 0.8 * 4,380 \mathrm{hrs} /\right.$ year $\left./ 1,000\right)=$ 12,100 klbs/year (Figure 3.8)

Method 2A - Based on design flow rates, pressure and pipe size:

A site has a central boiler plant that has two boilers discharging into a common header operating at 150 psig, 24 hrs per day for 6 months per year to distribute steam to several buildings. One proposed location for a meter is downstream of the plant, just before entering building \#1. The location is 455 feet downstream of the main plant. The line includes some fittings and elbows adding an equivalent pipe length of 10 percent, ${ }^{5}$ for a total of approximately 500 feet of 4 -in.-diameter piping (Figure 3.8).

\footnotetext{
${ }^{3}$ This value is a based on facilities using \#2 oil and/or natural gas.

${ }^{4}$ The boiler is operating at 100 percent rating when it is producing $34.5 \mathrm{lb} / \mathrm{steam}$ (boiler HP unit, $34.5 \mathrm{lb}$ evaporation per hr of equivalent steam from and at $212^{\circ} \mathrm{F}$ ).

${ }^{5}$ If the pipe is less than 50 meters (164 feet) long, add an allowance for fittings of 5 percent. If the pipe is over 100 meters (328 feet) long and is a fairly straight run with few fittings, an allowance for fittings of 10 percent would be made. A similar pipe length, but with more fittings, would increase the allowance towards 20 percent.
} 


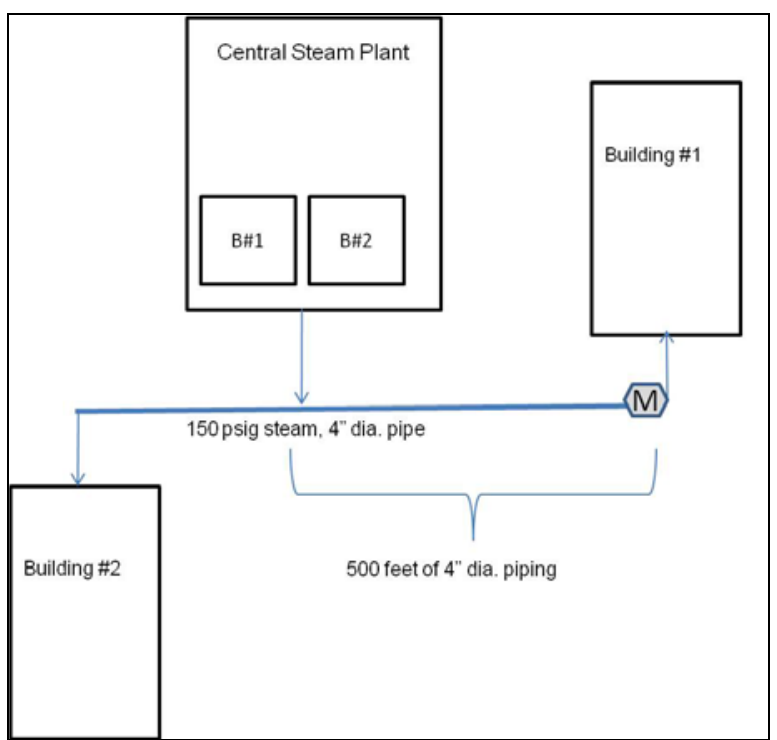

Figure 3.8. Central Steam Plant Schematic

- The following information is known (given in the example) because it can typically be determined by walking down the steam piping and/or reviewing design drawings or documents at the site:

- The steam pipe is 4-in. diameter, schedule 40 steel; the internal area of the pipe is a $0.08841 \mathrm{ft}^{2}$

- The line pressure upstream of the meter location is 150 psig, or 165 psia; thus, the specific volume of saturated vapor from the steam tables $=2.75$ cubic feet per pound $\left(\mathrm{ft}^{3} / \mathrm{lb}\right)$.

- The specific volume divided by the internal area of the pipe $=2.75 \mathrm{ft}^{3} / \mathrm{lb} /\left(0.08841 \mathrm{ft}^{2}\right)=$ $31.1 \mathrm{ft} / \mathrm{lb}$

- Based on engineering best practices, the recommended velocity for saturated steam ranges from 80 to 130 feet per second (fps). ${ }^{6}$ Therefore, using above given information and the conservative flow rate of 80 fps for saturated steam:

$$
=80 \mathrm{fps} / 31.1 \mathrm{ft} / \mathrm{lb}=2.6 \mathrm{lb} / \mathrm{sec} *(3,600 \mathrm{sec} / \mathrm{hr}) \sim 9,256 \mathrm{lb} / \mathrm{hr}
$$

- Using the same assumption for operating hours of 24 hrs per day for 6 months per year yields:

$$
\text { 9,256 lb/hr * 4,380 hrs/year/1,000 = 40,539 klbs/year }
$$

Method 2B - There are numerous graphs and charts that have been developed to replace the tedious equations which can also be used to estimate the flow rate based on pipe size and approximate pressure. For instance, based on the 150 psig, 80 feet per second, and a 4-in.-diameter pipe, the flow rate would be $8,500 \mathrm{lbs} / \mathrm{hr}$. However, to be more accurate, the pressure drop, heat loss and steam leaks, associated with a distribution system can be accounted for by applying generally accepted equations, utilizing graphs, and/or rules of thumb that have been developed to facilitate obtaining an estimate. The energy manager should decide based on the unique situation at their site what distribution losses to apply, and recalculate the flow rate as appropriate.

\footnotetext{
${ }^{6}$ Superheated steam can be considered dry gas, so no moisture to water droplets to erode the pipe, thus the steam can carry higher velocities; 165 to 230 fps.
} 
- The steam pressure drop due to the friction in the piping can be determined by using the graph based on the estimated steam flow rate and the pipe diameter (Figure 3.9).

- For example, using $80 \mathrm{fps}(9,256 \mathrm{lb} / \mathrm{hr})$, and the 4-in.-diameter piping, a 100-psig header will generate $\sim 1.9$ psig drop per $100 \mathrm{ft}$ of piping, and then applying the correction factor for a 150 -psig header, the $1.9 \mathrm{psig} / 100 \mathrm{ft} * 0.7=1.33 \mathrm{psig} / 100 \mathrm{ft}$. At 500 feet of piping (1.33 psig/ $100 \mathrm{ft}) * 500 \mathrm{ft}=6.65 \mathrm{psig}$, or the pressure at the meter is about 144 psig (150 psig $-6.65 \mathrm{psig})$.

- A rule of thumb, commonly used is if the line is insulated, the heat loss can be estimated at 3.5 percent per 100 meters (328 feet); therefore, (500 feet * 3.5 percent)/328 feet; 5.3 percent * $9,256 \mathrm{lb} / \mathrm{hr}=494$ feet. The 9,256 lb/hr $-494 \mathrm{lb} / \mathrm{hr}=8,762 \mathrm{lb} / \mathrm{hr}$.

- Distribution losses due to leaks are unique to each site and hard to estimate, a conservative estimate may be about 5 percent of the total steam generated; thus, 9,256 lb/hr - (5 percent * $9,256 \mathrm{lb} / \mathrm{hr})=463 \mathrm{lb} / \mathrm{hr}$ or $8,793 \mathrm{lb} / \mathrm{hr}$.

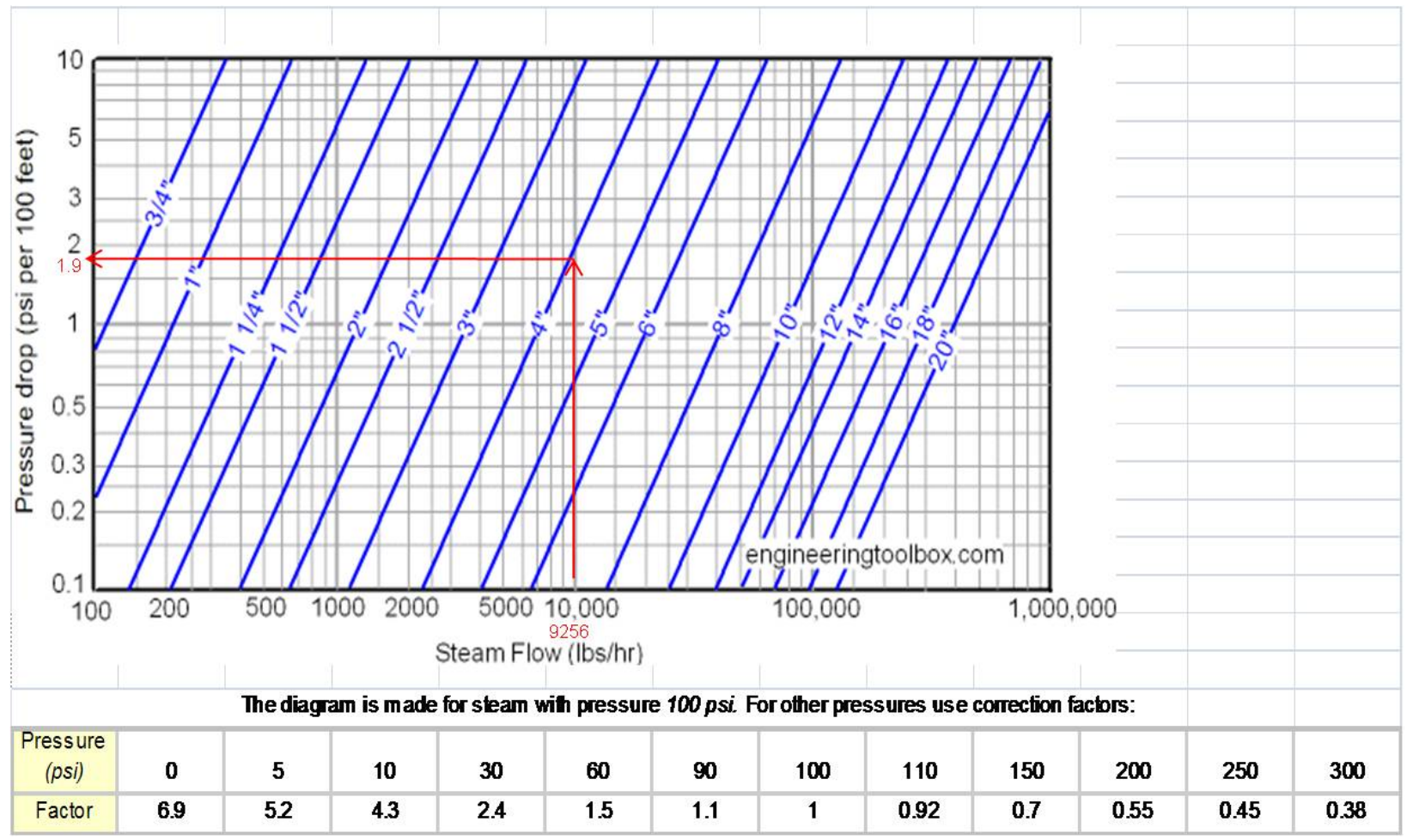

Figure 3.9. Steam Pressure Drop

Step Three - Calculate annual savings (S) or use Figure 3.10

Once the cost of steam, the annual steam consumption and expected savings are determined, the annual savings can be manually calculated or obtained from Figure 3.10.

- $\mathrm{C}_{\mathrm{S}}=\$ 10 / \mathrm{klb}$

- If the expected savings is $\sim 1$ percent on your investment, then...

- Expected annual savings $(S)=12,100 \mathrm{klbs} * \$ 10 / \mathrm{klbs} * 1$ percent $=\$ 1,210$. 
Note: Figure 3.10 provides a range from 1 percent to 5 percent of expected savings, depicting that savings increases as the steam use and cost of steam increases.

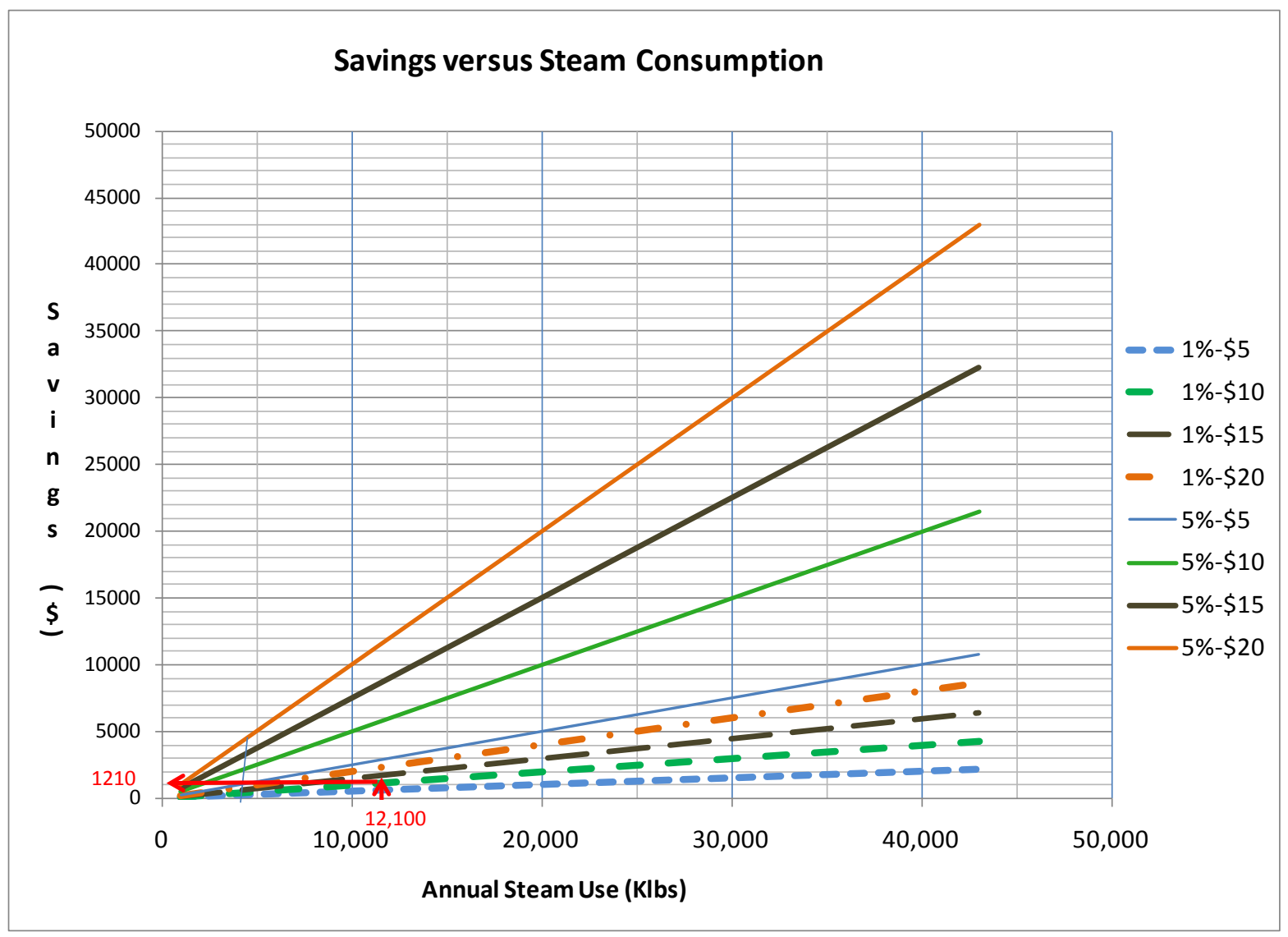

Figure 3.10. Savings Graph versus Annual Steam Consumption @ 1 Percent and 5 Percent Savings

Step Four - Case 1 - Calculate savings to investment ratio (SIR) or use Figure 3.11

Once the annual savings are determined, investment costs, annual operating costs are estimated, the SIR can be manually calculated or obtained from Figure 3.11.

Case 1 - Savings to investment ratio (SIR) calculation using investment costs (IC) $=\$ 2,000$

Annual savings $(\mathrm{S})=\$ 1,210$, and annual operating costs $(\mathrm{AOC})=\$ 500:\{\mathrm{PV}(\mathrm{S})-\mathrm{PV}(\mathrm{AOC})\} / \mathrm{IC}$ :

- Annual savings (S) are calculated above, and converted to a PV using:

- a 3 percent discount rate (i) per the National Institute of Standards and Technology (NIST) lifecycle costing analysis, and

- a conservative expected life (n) of 10 years. The life of a steam meter system (device and transmitter) is expected to be dependent on the application, with some systems in mild conditions (ambient temperatures, clean gas, etc.) having a mean time before failure over 30 years, while some in harsh environments failing in less than a year. 


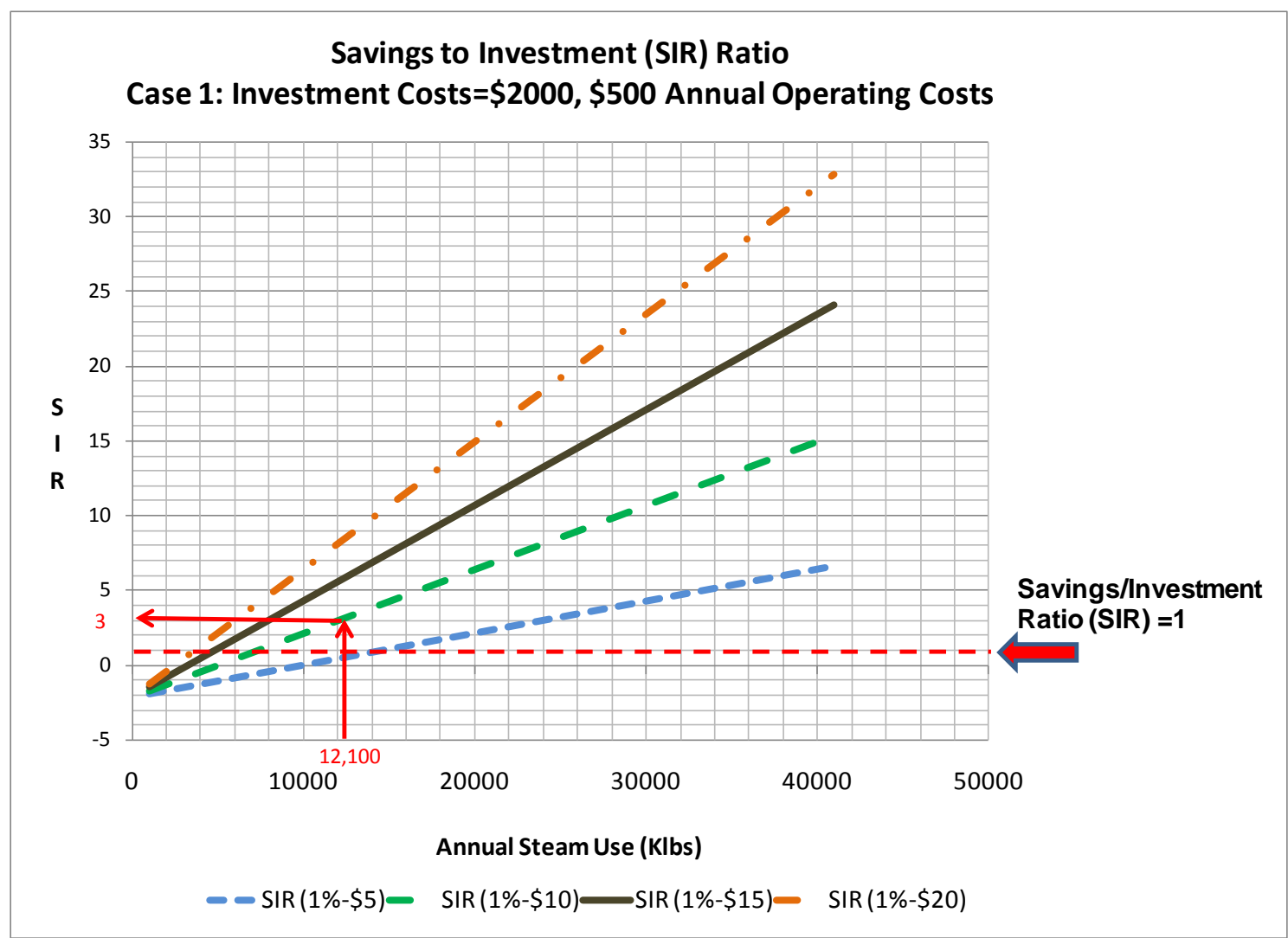

Figure 3.11. Case 1 - Steam Use of 12.1 klbs versus SIR Ratio @ 1 Percent Savings, \$2,000 Installed Cost

- Annual operating costs (AOC) are estimated at \$500/year; representing the costs to maintain (detailed in Section 5.3.4, and manage the metered data. These costs are also converted to PV using the same 3 percent discount rate and 10-year life

- At 3 percent rate (i) and 10-year life (n); P/A factor $=\left(\left((1+0.03)^{10}\right)-1\right) /\left(0.03 *(1+0.03)^{10}\right)=$ 8.5302

- The PV of annual savings (S) using P/A factor $=8.5302 * \$ 1,210=\$ 10,322$

- The PV of annual operating costs (AOC) using P/A factor $=8.5302 * \$ 500=-\$ 4,265$

- The PV of the net annual savings = PV( annual savings $)-P V($ annual operating costs $)=$ $\$ 10,322-\$ 4,265=\$ 6,056$

- The SIR ratio $=\$ 6,056 / \$ 2,000=3$.

Note that this SIR ratio $>1$; thus, the benefits outweigh the costs, and is a reasonable project.

Step Four - Case 2 - Calculate savings to investment ratio (SIR)

Once the annual savings are determined, investment costs, annual operating costs are estimated; the SIR can be manually calculated. 
Case 2 - Savings to investment ratio (SIR) calculation using investment costs (IC) $=\$ 4,000$;

Annual savings $(\mathrm{S})=\$ 1,210$ and annual operating costs $(\mathrm{AOC})=\$ 500:\{\mathrm{PV}(\mathrm{S})-\mathrm{PV}(\mathrm{AOC})\} / \mathrm{IC}$ :

- Example calculation of (SIR) ratio using the assumptions from above and calculating:

- At 3 percent rate (i) and 10-year life (n); P/A factor $=\left(\left((1+0.03)^{10}\right)-1\right) /\left(0.03 *(1+0.03)^{10}\right)=$ 8.5302

- The PV of annual savings (S) using P/A factor $=8.5302 * \$ 1,210=\$ 10,322$

- The PV of annual operating costs (AOC) using P/A factor $=8.5302 * \$ 500=-\$ 4,265$

- The PV of the net annual savings $=$ PV( annual savings $)-P V($ annual operating costs $)=$ $\$ 10,322-\$ 4,265=\$ 6,056$

- The SIR ratio $=\$ 6,056 / \$ 4,000=1.5$.

Note that again, the SIR ratio $>1$; thus, the benefits outweigh the costs, and is a reasonable project.

Step Four - Case 3 - Calculate savings to investment ratio (SIR) or use Figure 3.12

Once the annual savings are determined and investment costs and annual operating costs are estimated, the SIR can be manually calculated or obtained from Figure 3.12.

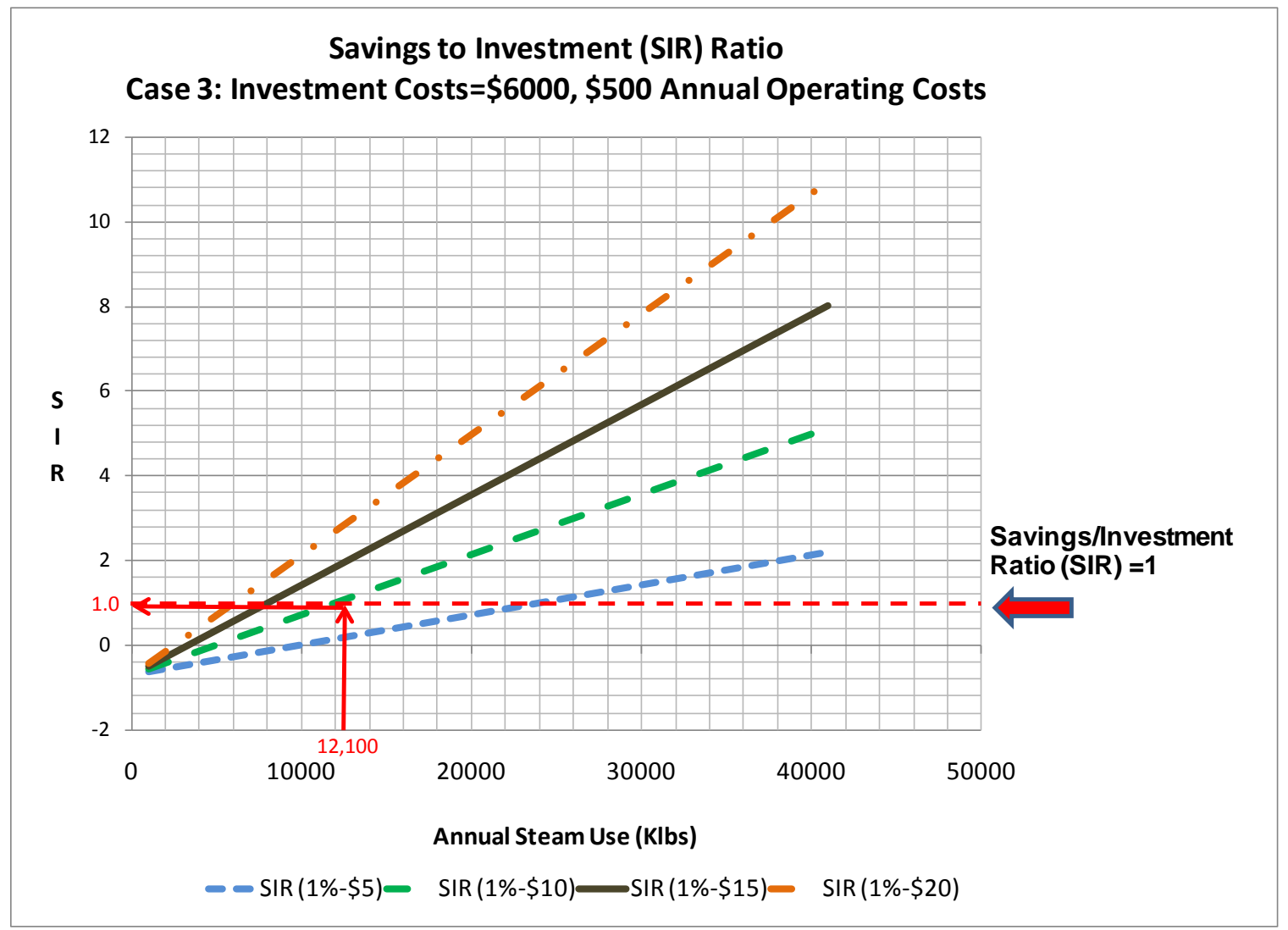

Figure 3.12. Case 3 - Steam Consumption of 12.1 klbs versus SIR Ratio @ 1 Percent Savings and \$6K Installed Costs 
Case 3 - Savings to investment ratio (SIR) calculation using investment costs (IC) = \$6,000;

Annual savings (S) = \$1,210 and annual operating costs (AOC) $=\$ 500:\{\mathrm{PV}(\mathrm{S})$-PV (AOC) $\} / \mathrm{IC}$ :

- Example calculation of (SIR) ratio using the assumptions from above and calculating:

- At 3 percent rate (i) and 10-year life (n); P/A factor $=\left(\left((1+0.03)^{10}\right)-1\right) /\left(0.03 *(1+0.03)^{10}\right)=$ 8.5302

- The PV of annual savings (S) using P/A factor $=8.5302 * \$ 1,210=\$ 10,322$

- The PV of annual operating costs (AOC) using P/A factor $=8.5302 * \$ 500=-\$ 4,265$

- The PV of the net annual savings $=$ PV( annual savings $)-P V($ annual operating costs $)=$ $\$ 10,322-\$ 4,265=\$ 6,056$

- The SIR ratio $=\$ 6,056 / \$ 6,000=1.0$.

Note that this project is at the SIR ratio $=1$; thus, the benefits equal the costs; thus, it would be prudent to review the costs to determine whether this project should be completed or not.

Therefore, the energy manager simply needs to determine which set of assumptions most represents their situation to determine whether the meter installation project is cost effective or the savings outweigh the costs.

\subsubsection{Water Metering}

Metering of water use is not currently mandated for Federal buildings. However, efficiency requirements outlined by EO 13423 for potable water and EO 13514 for industrial, landscaping, and agricultural applications have elevated the importance of monitoring and effectively tracking water-intensive applications. The intent of this section is to outline the considerations for the cost effectiveness of implementing water meters and to assist Federal agencies in making decisions on metering implementation. Further, it provides a means to evaluate when the annual cost savings of installing a meter offsets the initial and ongoing costs of the meter.

\section{Cost Justification}

To determine the relationship between the estimated costs and the estimated savings for a given payback period, the following cost justification methodology is applied:

$$
\text { Minimum Annual Water Bill }=\frac{\left[\left(\frac{\text { Installed Cost }}{\text { Desired Simple Payback }}\right)+\text { Annual Cost }\right]}{\% \text { Annual Savings }}
$$

where Minimum Annual Water Bill = the minimum annual cost of water for the given application or facility that is being considered for a water meter

Installed Cost $=$ the total cost to purchase, install, and commission the meter

Desired Simple Payback $=$ the number of years required for the metering system to achieve cost savings equal to the installed cost. In the Federal sector, 10 years is the maximum simple payback desired.

Annual Cost $=$ the annual meter maintenance, calibration, and data analysis 


\section{$\%$ Annual Savings = the estimated cost savings realized by implementing metering technologies. A value of 2 percent should be considered the conservative estimate, though more aggressive savings are considered, as well.}

Using this formula may require that there be a reasonable means of estimating the annual water bill for the building or sub-metered application being considered. If estimates are needed, the following methods may be applied:

- Square Footage - Estimate water use by dividing the total site use by the total site square footage, and multiplying this value by the square footage of the building being considered. (Note - this method may not provide accurate results. For example, a storage warehouse may have a larger water use than a commercial kitchen or a barracks with showers using this method, when the actual water use may be much higher for the kitchen and barracks.)

- Short-Term Metering - Annual estimates can be determined by applying meters temporarily to take short term readings and extrapolating the measured data into annual use levels. For instance, if a meter is installed for a three months, multiply the measured use by four to get an annual estimate.

- Other Estimating Methodologies - Additional estimating methodologies (one document focuses on industrial heating and cooling applications, along with wash stations, while the other document targets landscaping water use) can be used (PNNL 2010; DOE 2010).

\section{Data Inputs}

The following data has been used to provide graphical relationships of the minimum annual water bill against several different annual savings percentages for a variety of flow meters that are typically used in measuring building and process equipment water use (Figures 3.13-3.17):

- Capital Cost ranges were gathered from two different resources ${ }^{7}$ for several flow meter types that are typical for building water meters or sub-metered water applications. Prices for positive displacement, turbine, vortex shedding, and ultrasonic (portable and permanent) meters across a range of pipe sizes are detailed below:

- Positive displacement (1/4 in. to 2 in. pipe) - $\$ 1,000$ to $\$ 3,850$ ( $\$ 2,425$ average)

- Turbine (1/8 in. to 8 in. pipe) - \$278 to $\$ 1,997$ (\$1,137.50 average)

- Vortex shedding (1/4 in. to 4 in. pipe) - \$468 to $\$ 2,158$ (\$1,313.00 average)

- Portable ultrasonic (1/4 in. and larger pipe) - \$1,277 to \$6,279 (\$3,778 average)

- Permanent ultrasonic (2 in. to 6 in. pipe) - \$2,538 to \$3,112 (\$2,825 average)

- Installation Cost ranges for all meter types were obtained by gathering labor estimates from industry professionals and labor rates were obtained from current Bureau of Labor.

- Workers required -2

- Installation time (low) - 4 hours

- Installation time (average) - 6 hours

\footnotetext{
${ }^{7}$ From GSA (https://www.gsaadvantage.gov/advgsa/advantage/main/start_page.do) and Omega Engineering, Inc (http://omega.com/).
} 
- Installation time (high) - 8 hours

- 2011 hourly wages ${ }^{8}$ (weighted average) - \$18.40 (U.S.)

- Annual Cost ranges were obtained by gathering labor estimates from industry professionals and combined with the same labor rates as above.

- Workers required - 1

- Maintenance time (low) - 2 hours

- Maintenance time (average) - 4 hours

- Maintenance time (high) - 6 hours

- Data analysis time (low) - 4 hours

- Data analysis time (average) - 6 hours

- Data analysis time (high) - 8 hours

- Calibration time (low) -1 hour

- Calibration time (average) - 2 hours

- Calibration time (high) - 4 hours

- 2009 hourly wages $^{9}$ (weighted average) - \$55.35 (U.S.).

\section{Methodology}

Using the input data cited above and the earlier formula, the minimum water bill required to achieve annual savings of 2 percent, 3 percent, 4 percent, and 5 percent was computed. This range of annual water savings is based on achievable water savings gained from better management of water use through analysis of metered data. For example, flow meters can reveal operational problems such as equipment, distribution, or building water leaks.

Three different price ranges were trended for each meter type listed (positive displacement, turbine, vortex shedding, and portable ultrasonic and permanent ultrasonic). ${ }^{10}$ The highest meter prices are represented by the highest capital cost, high installation time multiplied by the 2009 weighted average hourly wage, and high maintenance time multiplied by the 2009 fully burdened hourly wage. Low price trends for each meter are represented by the lowest capital cost, low installation time multiplied by the 2009 fully burdened hourly wage, and low maintenance time multiplied by the 2009 fully burdened hourly wage. Similarly, average price trends are also included to provide a high, low, and average range to consider at what point installing a meter becomes cost-effective. For each scenario the desired simple payback is 10 years.

\section{Results}

The following series of charts display curves that represent the breakeven point of where the cost of installing and maintaining a water meter is equal to the annual cost savings achieved. The charts can be used to help determine if it makes economic sense to install a flow meter. This can be done by locating

\footnotetext{
${ }^{8}$ From Bureau of Labor Statistics real wages by state for Installation, Maintenance, and Repair workers projected through 2011 using the Bureau of Labor Statistics real wage growth rate.

${ }^{9}$ From Bureau of Labor Statistics Occupational Employment wages for plumbers, pipefitters, and steamfitters. Rate was then indexed against fully burdened labor rates published by the Los Angeles School District and weighted against U.S. Census Bureau populations for each state.

${ }^{10}$ Other meters can be assessed with these trends if their capital cost is similar to any of the five categories provided.
} 
the percent annual savings (on the x-axis) that is reasonable for the application and then tracing up from this point to the corresponding curve on the chart for the meter cost that best represents the meter installed cost of the application - high, average, or low costs (see costs in "Data Inputs"). Then from this cost curve, trace over to the corresponding minimum annual water bill on the y-axis. This value represents the minimum water cost of the application that will achieve an economic breakeven point.

\section{Positive Displacement Meters}

Figure 3.13 shows that at 2 percent expected annual savings, a positive displacement meter with a high installed cost requires a minimum annual water bill of $\$ 73,493$ to be cost-effective. While a meter with an average installed cost requires a minimum annual water bill of $\$ 48,656$ and a low-cost meter requires a minimum annual bill of $\$ 26,587$ to be cost-effective. But, if the annual savings is expected to be greater than 2 percent, then the minimum annual water bill required to reach cost-effectiveness will be dramatically lower. It is also interesting to note that as the expected annual savings increases, the three installed cost curves for high, average, and low costs tend to converge. For example, if a 5 percent annual savings is expected, then the minimum annual water bill required for cost-effectiveness decreases to between $\$ 29,397$ and $\$ 10,635$. Considering that a 2 percent annual savings is fairly conservative and higher savings are likely achievable, many metering applications will be cost-effective at Federal installations.

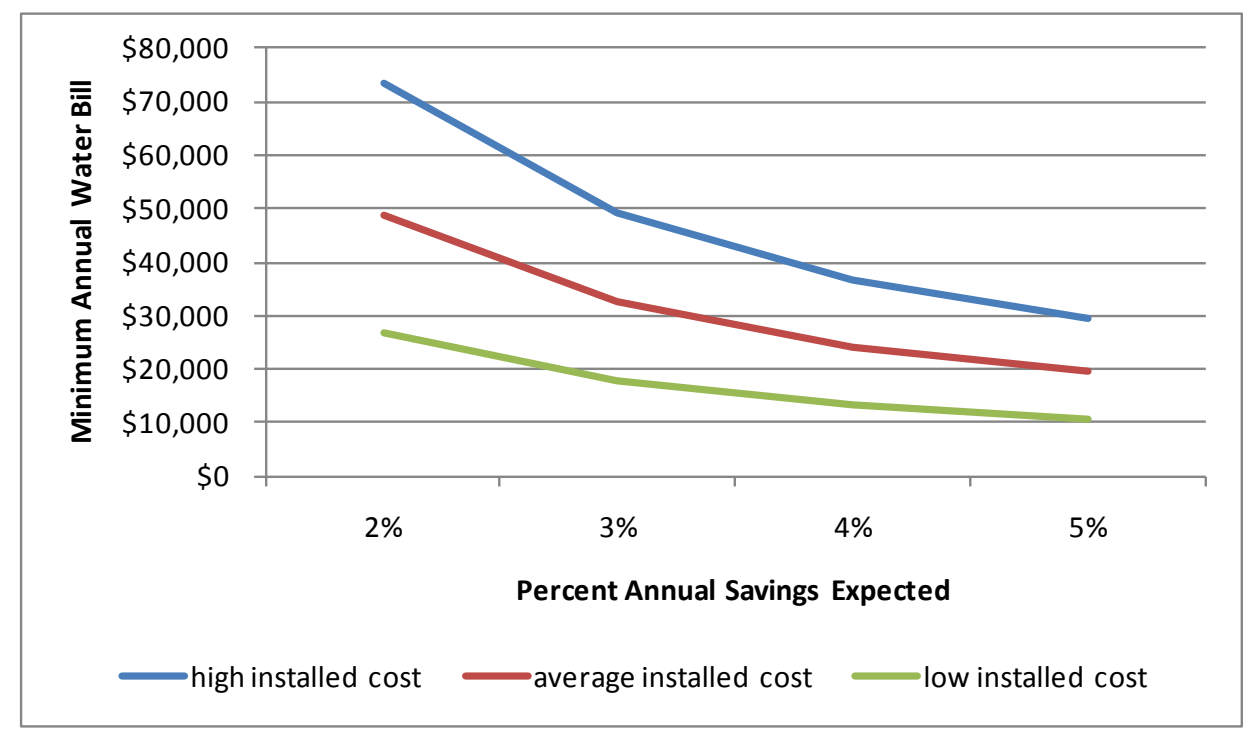

Figure 3.13. Cost-Effectiveness of Positive Displacement Meters

\section{Turbine Meters}

Figure 3.14 shows that at 2 percent expected annual savings, a turbine meter with a high installed cost requires a minimum annual water bill of $\$ 64,228$, an average cost meter requires a minimum annual water bill of $\$ 42,219$, and a low-cost meter requires a minimum annual bill of $\$ 22,977$ to be cost-effective. As the expected annual savings increases by fully utilizing the benefits of metering, the minimum annual water costs dramatically decrease making many applications of turbine meters cost-effective. For example, if a 5 percent annual savings is expected, then the minimum annual water bill required for costeffectiveness decreases to $\$ 25,691$ on the high end and $\$ 9,191$ for the low-cost scenario. 


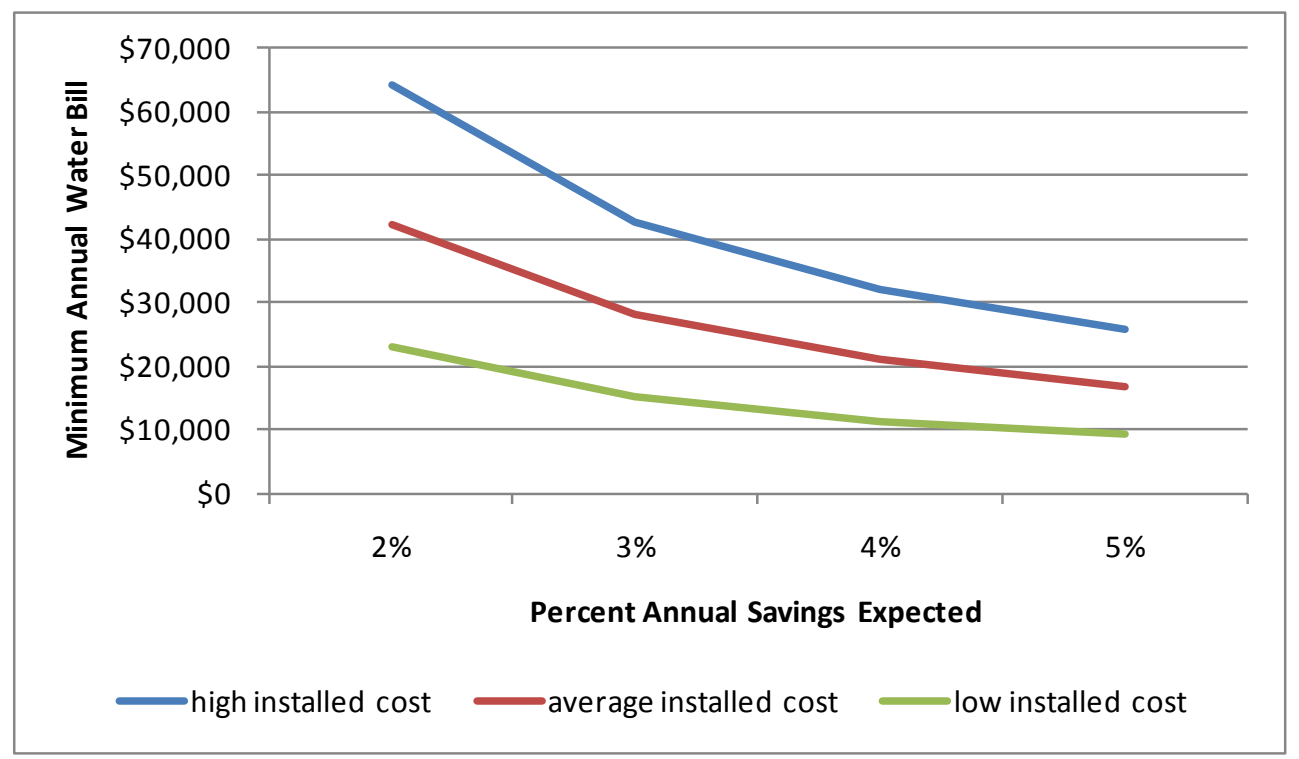

Figure 3.14. Cost-Effectiveness of Turbine Meters

\section{Vortex Shedding Meters}

For vortex shedding meters, Figure 3.15 shows that at 2 percent expected annual savings, a vortex shedding meter with a high installed cost requires a minimum annual water bill of $\$ 65,033$, an average cost meter requires a minimum annual water bill of $\$ 43,096$, and a low-cost meter requires a minimum annual bill of $\$ 23,927$ to be cost-effective. If a 5 percent annual savings is expected, then the minimum annual water bill required for cost-effectiveness decreases to $\$ 26,013$ at the high end and $\$ 9,571$ for the least expensive consideration.

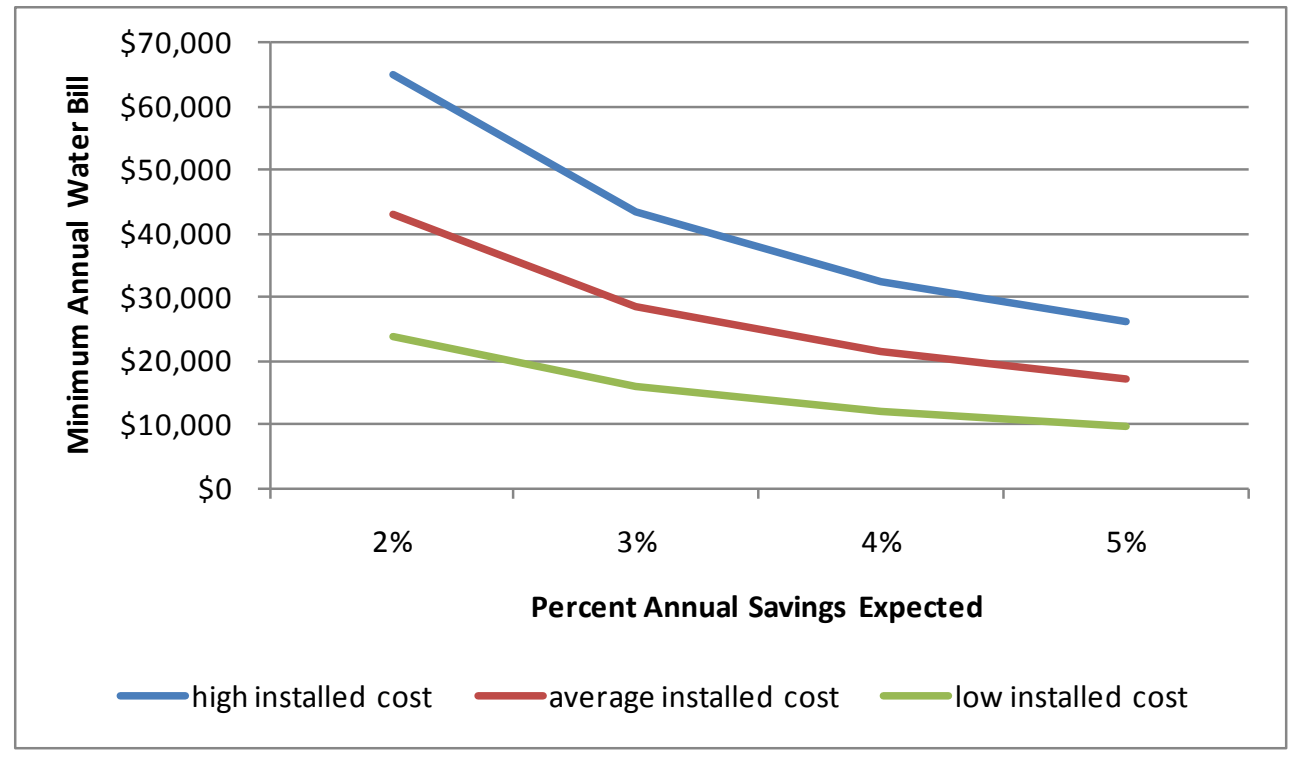

Figure 3.15. Cost-Effectiveness of Vortex Shedding Meters 


\section{Portable Ultrasonic Meters}

Figure 3.16 shows that at 2 percent expected annual savings, a high-cost portable ultrasonic meter requires a minimum annual water bill of $\$ 85,638$, an average cost meter requires a minimum annual water bill of $\$ 55,421$, and a low-cost meter requires a minimum annual bill of $\$ 27,972$ to be cost-effective. If a 5 percent annual savings is expected, then the minimum annual water bill required for cost-effectiveness decreases to $\$ 34,255$ for the most expensive scenario and $\$ 11,189$ at the low-cost price range.

(Note: Portable ultrasonic meters are intended for temporary use and may be used on multiple applications. The breakeven costs curves are useful to determine whether a temporary meter is justified, but this type of meter would ultimately be replaced by a less expensive permanent meter.)

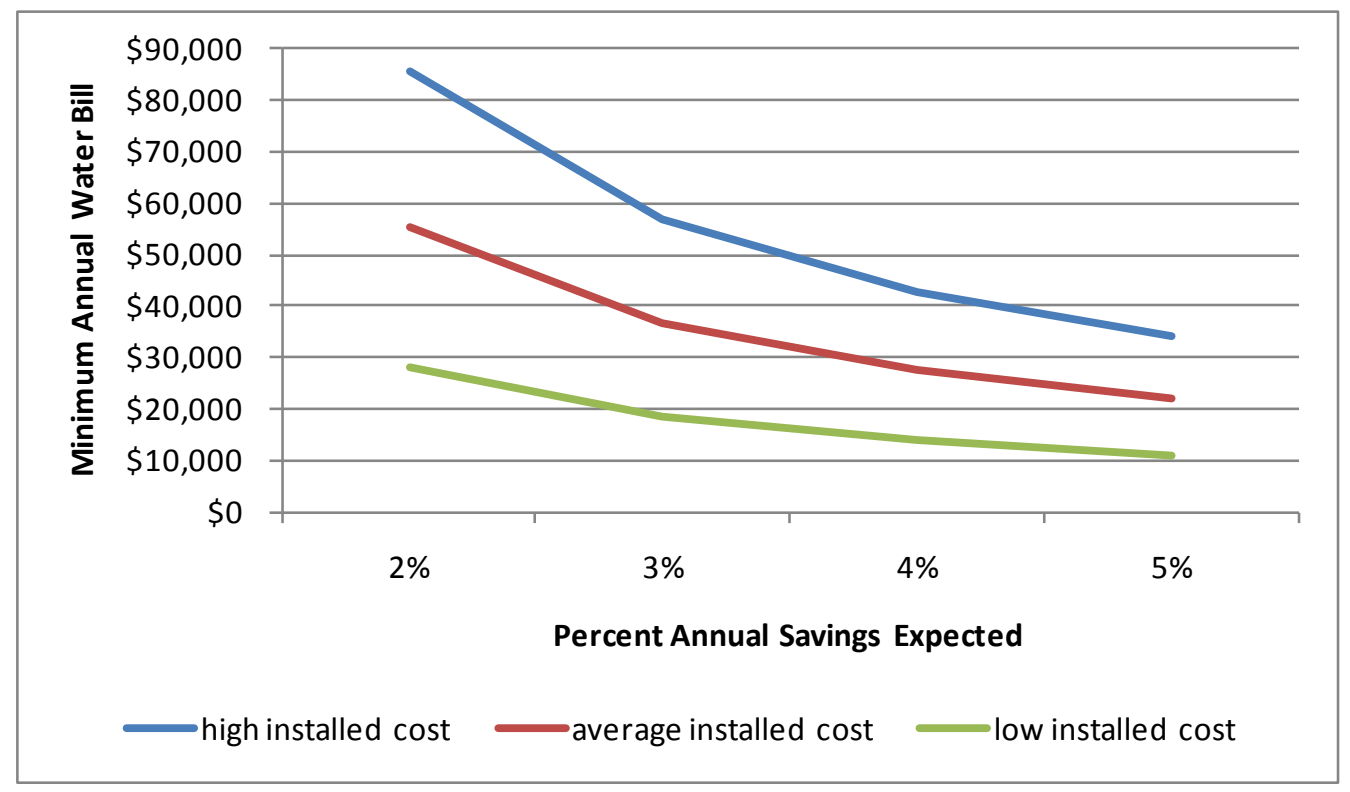

Figure 3.16. Cost-Effectiveness of Portable Ultrasonic Meters

\section{Permanent Ultrasonic Meters}

Figure 3.17 shows at 2 percent expected annual savings, a high-cost permanent ultrasonic meter requires a minimum annual water bill of $\$ 69,803$, an average cost meter requires a minimum annual water bill of $\$ 50,656$, and a low-cost meter requires a minimum annual bill of $\$ 34,277$ to be cost-effective. If a 5 percent annual savings is expected, then the minimum annual water bill required for cost-effectiveness decreases to $\$ 27,921$ for the high-cost meter and $\$ 13,711$ for the least expensive option. 


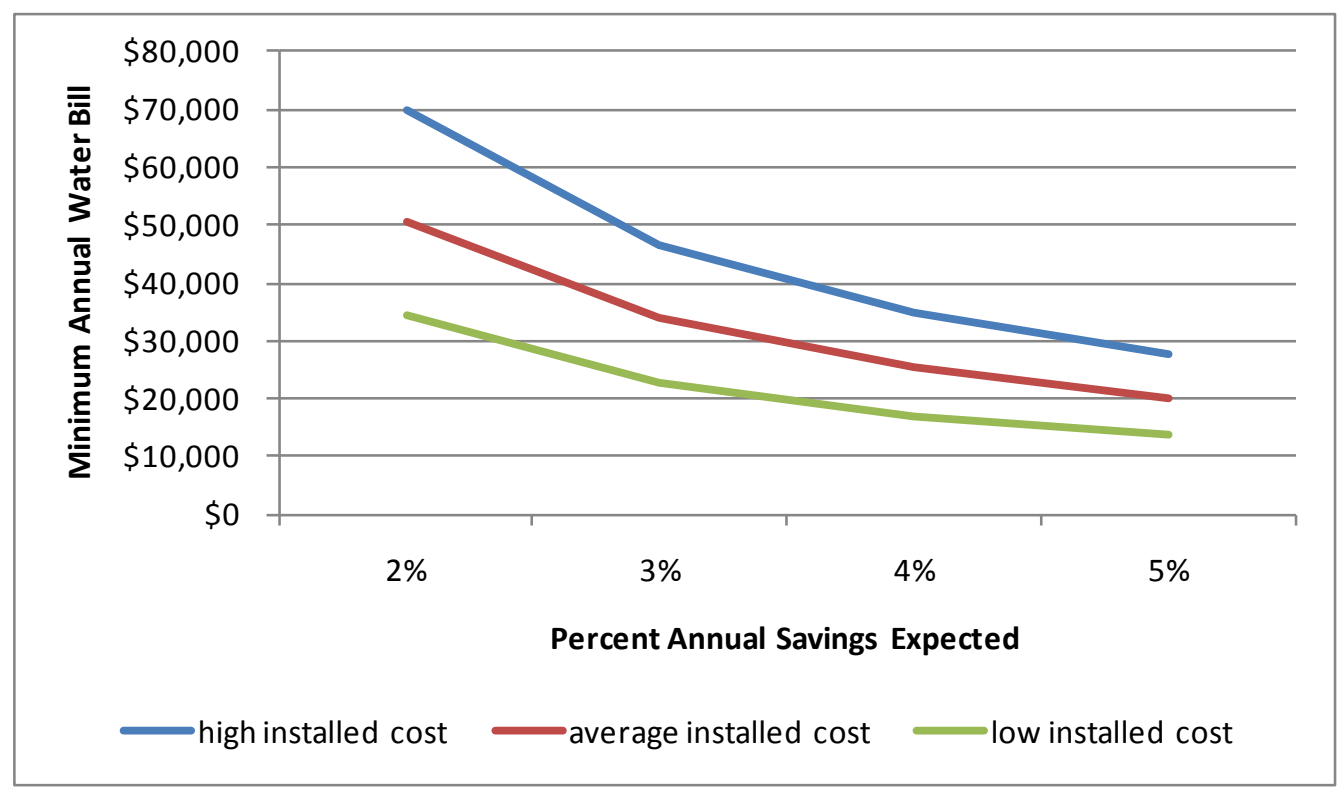

Figure 3.17. Cost-Effectiveness of Permanent Ultrasonic Meters

\subsubsection{Financing Options}

Identifying the proper financing approach is a critical step in the successful implementation of a metering program. The selected financing option, or options, will impact the overall system design and its capabilities, as well as the installation timeline. Since there are many financing options available to the Federal sector, the financing approach used at a given site should be selected based on site-specific needs and opportunities.

\subsubsection{Metering System Purchasing and Installation Financing Approaches}

There are a number of potential financing alternatives available to Federal sites. Factors affecting the financing alternatives available include estimated system cost, agency policies, and utility company support offerings, to name a few. In some cases, sites will be able to finance their metering systems through a combination of approaches, while in other cases they may be limited to single options.

\section{Factors affecting the} financing alternatives

available for metering include estimated system cost, agency policies, and utility company support offerings.

\subsubsection{Metering Financing Hierarchy}

As a way for sites to begin their initial considerations of financing alternatives, the financing hierarchy has been developed (Figure 3.18). This hierarchy is based on life-cycle costs to the site's facility, utility, or energy management program as many consider life-cycle costs to be the most significant factor in selecting their financing approach. Additional hierarchies may be developed based on factors such as speed of implementation or lowest first/up-front cost. Note that a lowest first cost approach may allow for faster implementation or a metering program with expanded capabilities. 


\section{The Metering Financing Hierarchy}

"No-Cost" Options:

- Policy-directed approaches

- Include in construction and renovation projects

- Assess tenant fees

- Reinvest energy savings

- Utility provided (for time-based rates customers)

Appropriations Options:

- Line item appropriations

- Locally managed appropriations

Alternative Financing:

- Utility energy service contracts (UESC)

- Energy savings performance contracts (ESPCS)

Figure 3.18. The Metering Financing Hierarchy

Two key assumptions are applied to this hierarchy:

- Recurring costs (system operations and maintenance) are the same for all of the approaches.

- Resulting energy savings are the same regardless of the financing approach.

The approaches addressed at the top of the hierarchy are the so-called "no cost" options. This is not to imply that the meters are free; instead, the costs to purchase and install the meters are covered in part or in total by programs other than the site facilities, utilities, or energy program.

Including the

up-front and/or installation costs of

metering in

The appropriations approaches are next in this hierarchy's order. While the costs for the metering system are now being incurred by the site or agency facilities programs, agency appropriations are a familiar approach where the total costs of the meters are paid at the time of purchase and installation.

construction and renovation projects is recommended

for all agencies

and sites.

Alternatively financed approaches appear at the bottom of this hierarchy since the overall life-cycle costs are higher than the appropriations approaches due to added financing charges. This is not to imply that these approaches are any less likely to succeed than the other options. Site metering opportunities at some sites may benefit from alternative financing as a way expand the metering system or add capabilities beyond the EPAct 2005 requirements.

\subsubsection{Descriptions of Financing Approaches}

The first options to consider are those for which the up-front purchase and/or installation costs of metering are covered entirely or in part by funding sources outside the (direct) facilities, utilities, or energy program budgets. 


\section{Include in Construction and Renovation Projects}

- Description: Require by site (or agency) policy that meters be included in all new building construction and major renovation projects.

- Benefits:

- The cost of the meter is assumed as a (very) small part of the overall project budget

- A separate metering project is not required for new buildings

- Disadvantages:

- Meters might be lost to value-engineering. Metering program manager must track inclusion of meters from start of project (initial funding request) through completed construction.

- New construction represents a very small part of the Federal building inventory on an annual basis. Sites will still need to address metering requirements at remaining buildings.

- Where to use: This approach is recommended for all agencies and sites.

- Additional considerations: The DoD Instruction 4170.11 (DoD 2009) establishes the requirement that meters be included in all new building and major renovation projects exceeding $\$ 200,000$. Metering requirements cover electricity, natural gas, and water.

\section{Assess Tenant Fees}

- Description: Include the cost to purchase, install, maintain, and operate the metering system as part of the tenant rental rates.

- Benefits:

- Tenant assessments are already in place at many sites

- Meters will allow for billing tenants for their actual energy/utility use. Tenants will then have greater incentive to reduce use.

- Funding approach can address post-installation metering system operations and maintenance financing requirements.

- Disadvantages: Tenant buy-in for additional assessment fees may be difficult to obtain.

- Where to use: Multi-tenant sites where utility cost allocation is being practiced or considered.

- Additional considerations: This approach can also be used in at multi-tenant single building sites.

\section{Reinvest Energy Savings}

- Description: Per EPAct 2005, agencies may retain funds not expended because of energy or water savings, provided that these funds further promote energy efficiency, water conservation, or unconventional and renewable energy resources projects. Metering equipment leading to additional energy savings is then an eligible

Reinvesting energy savings in a metering program can offset the requirements for appropriations funding. investment for retained energy savings.

- Benefits:

- Reinvested energy savings can offset the requirement for appropriations funding.

- Competition for these funds is limited to energy and water efficiency measures. 
- Disadvantages:

- Retention of savings will be addressed on an agency-by-agency basis.

- Sites will likely need to document sources of savings when determining funds available for retention. Some agencies will not retain the savings since EPAct 2005 only allows, but does not require the savings to be retained.

- Where to use: When these funds are available and when the metering system is being installed using appropriated dollars.

- Additional considerations: From a planning perspective, it will be difficult to estimate future funding streams available for reinvestment because funding amounts will be impacted by a number of factors such as other ongoing efficiency measures, weather, and operational requirements.

Retention of Energy and Water Savings
EPAct 2005, Section 102, paragraph (f), Retention of Energy and Water Savings,
amended section 546 of the National Energy Conservation Policy Act (42 USC 8256) as
follows:
"(e) Retention of Energy and Water Savings.-An agency may retain any funds
appropriated to that agency for energy expenditures, water expenditures, or
wastewater treatment expenditures, at buildings subject to the requirements of
section 543(a) and (b) [energy reduction goals], that are not made because of
energy savings or water savings. Except as otherwise provided by law, such
funds may be used only for energy efficiency, water conservation, or
unconventional and renewable energy resources projects. Such projects shall
be subject to the requirements of section 3307 of title 40, United States Code
[Congressional approval of proposed projects]."
While EPAct 2005 allows agencies to re-apply these funds for additional energy and water
efficiency measures, it is up to each individual agency to determine if such a
re-investment will be allowed and any procedures to be followed.

\section{Utility-Provided Meters}

- Description: Customers requesting to be placed on time-based electric rate schedules may receive an advanced meter from their servicing utility.

- Benefits: Meter is provided at no cost.

- Disadvantages:

- Time-based rates are not universally available.

- Time-based rates may not be the best rate tariff option available to the site.

- Customers will likely be limited to one meter per account.

- Where to use: Use at all sites where a time-based rate tariff is or will be in

Data analysis may be provided by the utility; however, sites may still need to complete their own data analyses to support sitespecific data use activities. effect. 
- Additional considerations:

- Site may need to work with the utility to access the data in real-time or near-real-time.

- Data analysis may be provided by the utility; however, sites may still need to complete their own data analyses to support site-specific data use activities.

- Caution: Do not disaggregate loads at multi-building sites for the purpose of obtaining additional meters from the electric utility as this would work to create multiple smaller customers that would be subject to less favorable rates.

Time-Based Rates (DOE 2006) (EPAct 2005)

Section 1252 of EPAct 2005, Smart Metering, requires that within 18 months of enactment [by February 2007] that states investigate and decide whether to mandate utilities to offer to each customer a time-based rate schedule under which the rate charged by the electric utility varies during different time periods and reflects the variance, if any, in the utility's costs of generating and purchasing electricity at the wholesale level. The time-based rate schedule would enable the electric consumer to manage energy use and cost through advanced metering and communications technologies. If the states mandate time-based rate schedules, each electric utility would provide each customer requesting a time-based rate with a time-based meter capable of enabling the utility and customer to offer and receive such a rate, respectively.

Types of time-based rates include

- Time-of-Use (TOU) Pricing: Energy prices that are set for a specific time period on an advance or forward basis, typically not changing more often than twice a year (summer and winter season). Prices paid for energy consumed during these periods are pre-established and known to customers in advance of such consumption, allowing them to vary their demand and usage in response to such prices and manage their energy costs by shifting usage to a lower cost period, or reducing consumption overall. The time periods are pre-established, typically include from two to no more than four periods per day, and do not vary in start or stop times.

- Critical Peak Pricing: A type of dynamic pricing whereby the majority of kWh usage is priced on a time-of-use basis, but where certain hours on certain days where the system is experiencing high peak demand are subject to higher hourly energy prices that reflect market conditions for peak generation and delivery during peak demand periods. These critical period prices may be known to electricity customers under conditions as "day-ahead" or "hour-ahead" and are typically employed a limited number of times per year.

- Real-Time Pricing: Energy prices that are set for a specific time period on an advance or forward basis and that may change according to price changes in the generation spot market. Prices paid for energy consumed during these periods are typically established and known to consumers a day ahead ("day-ahead pricing") or an hour ahead ("hour-ahead pricing") in advance of such consumption, allowing them to vary their demand and usage in response to such prices and manage their energy costs by shifting usage to a lower cost period, or reducing consumption overall. 


\section{Line Item Appropriations}

- Description: Metering projects are funded through the agency's line item appropriation. Funds are then distributed to the sites by the agency.

- Benefits:

- Federal agencies and sites are familiar with this funding approach.

- There are no long-term commitments for repayment or performance-based requirements that must be monitored.

- Disadvantages:

- Metering must compete against other initiatives for funding.

- It can take several years from the time of the initial funding request to the time that the funds are actually available to the site.

- Initial cost estimates are important as funds received set an overall cost cap.

- Where to use: Use at sites as directed by the agency.

- Additional considerations:

- Federal sites should inquire within their agencies if line item appropriations will be sought and when they will be available. Several agencies have adopted this approach - the General Services Administration and the U.S. Department of the Army in particular.

- Agency appropriations may apply meeting certain requirements such as EPAct 2005 (building electric), EISA 2007 (natural gas and steam), or DoD Instruction 4170.11 (DoD 2009). Metering programs that intend to address metering beyond the legislated or mandated requirements may need to identify additional funding sources.

\section{Locally Managed Appropriations}

- Description: Sites use funds from locally managed accounts such as utilities or small projects.

- Benefits:

- Federal sites are familiar with this funding approach.

- The level of effort required to obtain local funding, especially "small” amounts, may be easier and faster than obtaining funds from the other available approaches.

There are no long-

- Appling locally managed funds allows for incrementally building a metering system.

- Disadvantages: Metering projects must compete against other local projects for funding.

- Where to use:

- Especially well-suited for sites where metering costs are expected to be small.

- Do not use local funding if agency line item funding is available unless additional funds are needed to increase system capabilities (i.e., equipment monitoring, building sub-metering, and utilities other than term commitments for repayment or performance-based requirements that must be monitored with line item appropriations for funding metering projects. electricity). 
- Additional considerations: Agencies may prefer this approach over the line item approach since using locally managed funds allows the sites to prioritize metering against other facilities needs.

\section{Utility Energy Service Contracts (UESCs)}

- Description: Servicing utility company finances the purchase of the meter or services in support of a metering program internally or through a third party, with repayment back to the utility as part of the monthly bill, or directly to the third party.

- Benefits:

- Over the years, many Federal sites have installed energy efficiency measures by using financing UESC.

- Financing for projects and services can be used for small projects (several hundreds of dollars) and large projects (millions of dollars) alike.

- Services supporting other metering program needs can also be financed:

- Engineering services such as surveying existing systems and designing new systems.

- The purchase and installation of meters.

- Monthly services including meter maintenance and data analysis.

- Several approaches are available to Federal sites that want to work with their servicing utility: Area-wide contracts, basic ordering agreements, and rebates. Visit the FEMP Utility program website at http://www1.eere.energy.gov/femp/financing/uescs_types.html for a description of the available utility financing approaches.

- Disadvantages:

Always take
advantage of
available rebates that
support your metering
program goals and
objectives when it
comes to utility
company financing.

Always take

advantage of

support your metering

program goals and

objectives when it

comes to utility

company financing.
- UESC projects will be assessed interest fees.

- Not all utilities offer UESC.

- Sites need to make sure that money is available to cover the added financing cost included in the utility bill. Ideally, these funds will be available from the offsets realized by reduced utility cost savings.

- Where to use:

- Always take advantage of available rebates that support your metering program goals and objectives.

- Consider using when appropriations are not available or if the availability of utility financing presents a significant time advantage (accelerated savings) over appropriated dollars.

- Additional considerations: Sites may want to consider financing the installation of a metering system in conjunction with additional energy efficiency projects when a UESC is used.

\section{Energy Savings Performance Contracts (ESPCs)}

- Description: Install meters/metering system as part of an ESPC. There are two approaches by which this can be accomplished:

- Install the meters as an energy conservation measure (ECM) under the ESPC. In this case the savings will be stipulated since the resulting savings occur in response to the metered data and its analysis, not the meters themselves. 
- Install the meters in support of or as part of an ECM. Key to this approach is ensuring that energy savings are realized and that they can be verified. Examples of this approach include

- Using interval data as part of an ongoing retro-commissioning activity

- Using interval data to support of actions completed by the ESPC contractor such as real-time purchasing, peak load management, equipment diagnostics, and electric rate comparisons.

- Benefits:

- ESPCs are available to all Federal sites.

- The ESPC contractor can assume the data analysis function and reporting.

- Disadvantages:

- ESPC costs include finance charges, contract overhead fees, and measurement and verification expenses.

- ESPCs typically require an overall project investment of no less than $\$ 500,000$ dollars.

ESPCs are
available to all
Federal sites.
Consider using an
ESPC when
appropriated funds
or UESC are not
available.

- Where to use:

- Consider using an ESPC when appropriated funds are not available

- ESPCs are well suited for Federal sites that want to include a number of ECMs, especially if the site would like to have all the metering program functions contracted.

- Additional considerations:

- It is strongly recommended that the ESPC assign to the contractor the data analysis functions, and that there are provisions for taking actions resulting from the analysis findings and recommendations.

- While the measurement and verification (M\&V) for some ESPCs requires metering, this metering is typically applied to specific equipment. In these cases, the metering system should incorporate this metered data provided it supports a metering program objective. However, M\&V metering can support the EPAct 2005 requirements only when whole-building electrical metering is used, and likewise for EISA 2007 requirements for natural gas and steam metering.

- $\quad$ The National Institutes of Health in Bethesda, Maryland (Figure 3.19) embarked on a complex-wide ESPC focused on meters and metering systems. This activity is highlighted in the Case Studies section (Chapter 8) of this guide. Visit the Federal Energy Management Program's ESPC website for information on ESPCs: http://www1.eere.energy.gov/femp/financing/ espcs.html.

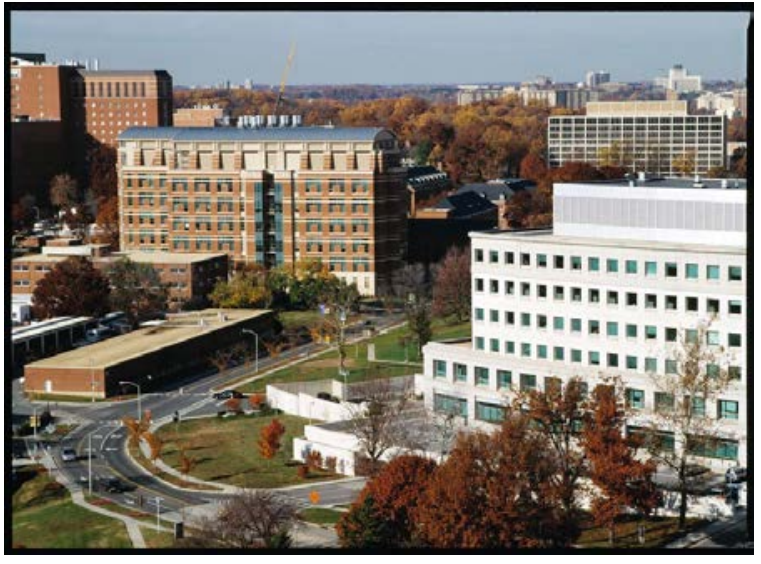

Figure 3.19. The National Institutes of Health in Bethesda, Maryland, is using an ESPC to install over 350 meters across the complex. 


\subsubsection{Selecting a Financing Approach}

Selecting a financing approach is site specific as there are a number of factors that will influence the decision:

- What are the site's metering needs?

Selecting a financing approach is site specific as there are a number of factors that will influence your decision.
- Are there agency policies affecting systems capabilities such as reporting requirements and financing approaches? For example, some agencies are financing their installations through line item appropriations, while others may direct the sites to first contact their local utility to see if there are any financial or technical assistance offerings.

- What is the current and projected availability of funds at the agency and site levels?

- What financial and technical assistance does the servicing electric utility offer?

- Does the site have the staff resources to commit to completing data analyses in a timely manner? What about the ongoing repair and maintenance of the metering system components?

- Does it make sense to consider installing a metering system as part of a larger site-wide energy efficiency project?

\subsubsection{Metering System Operations and Maintenance Funding}

The success of the metering program depends on the quality of the data collected and its productive application.
The up-front costs to purchase and install a metering system tend to generate the most attention when sites are planning their metering programs. However, the success of the metering program depends on the quality of the data collected and its productive application. Sufficient funding for the continuing operations and maintenance of the metering program must be in place once the metering system components are in operation. Funds to support the metering program will likely need to be identified from the facilities operations budget, and will cover a range of expenses including the ongoing operation of the meters, data storage, data analysis, and reporting/communications.

\subsection{Interval Data and Energy Profiles}

Energy interval data can be defined as time-stamped energy usage values recorded at regular periods. The value of interval data is the consistent recording of energy use with shorter intervals (e.g., 15 minute) offering increased resolution and diagnostic capability. Most advanced meters and smart meters are capable of at least hourly intervals and many record data at the utility-standard 15-minute interval.

Whole-building interval data contain a wealth of information on building energy use, both real-time and over a duration (e.g., seasonally, monthly, weekly, or daily). Interval data should be graphically represented and used to identify energy use peaks and spot trends that indicate problems such as equipment coming online earlier or running longer than expected (Better Bricks 2011). 
Figure 3.20 presents a daily demand profile for a commercial office building generated from hourly interval data. Evident from these data are three distinct regimes of energy use:

- Energy use that occur 24-hours per day, with a similar pattern each day (some variation on weekends) is defined as base load energy use. This minimum energy use occurs when the building is both occupied and unoccupied and is usually made up of security lighting, ventilation, and plug loads that operate continually.

- Energy use that tracks occupancy is referred to as the variable load. These loads are typically proportional to occupancy and are made up of lighting, computers, office equipment, and any convenience equipment (e.g., coffee makers and other cooking equipment).

- The final regime of energy use is the weather-dependent load. This energy use is proportional to outside air temperature (and humidity) and has notable seasonal variability.

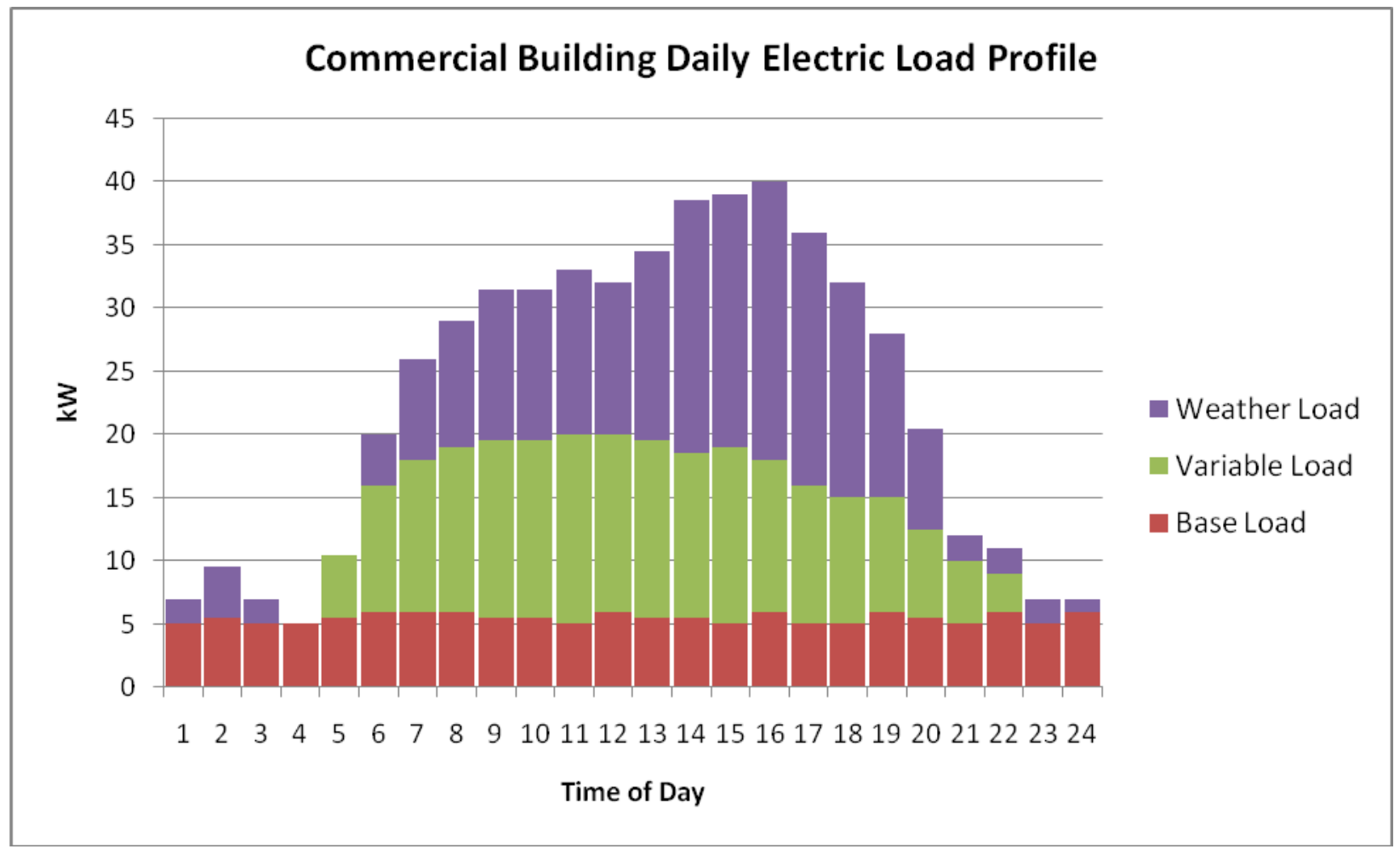

Figure 3.20. Typical Commercial Building Daily Electric Load Profile

Daily interval load data graphs should be generated and viewed/analyzed to identify operational issues. These graphs are particularly useful in verifying expected profiles of weekday versus weekend energy use; heating, cooling, and shoulder season energy use, and validating the relative shares of base load, variable load, and weather-dependent loads. Questions to be asked/answered with these data include:

- When do peak loads occur?

- Is the base load energy use within expectation?

- Does the overall profile match operating hours? 
- How does weather affect total energy use?

- Are HVAC controls set properly to take advantage of conditions and temperature set-back opportunities?

- Are seasonal variances within expectation?

- What are the causes of out-of-range values?

- What opportunities are there to reduce or change the shape of the profile?

- How does this profile and energy use compare with similar buildings?

Load profiles should be automatically generated and presented to relevant managers and practitioners on a daily basis.

Seasonal loads will have characteristic shapes depending on the building type, age, hours of operation, and fuels used. If natural gas is used as a heating fuel, similar interval data should be obtained and profiles developed. Seasonal comparisons should be made with knowledge of variance in weather conditions; weather normalization is recommended if seasonal comparisons are to be made.

In addition to standard spreadsheet tools for analysis, there are a number of off-the-shelf and custom application services provided by vendors. Chapter 7 provides additional information on data analysis and vendor offerings/contacts.

\subsection{Data Collection, Loss, Tracking, and Security}

\subsubsection{Data Collection}

Meters that comply with EPAct 2005 and EISA 2007 will have data accessibility on a daily basis. Daily (if not hourly) data collection and processing is recommended for the following reasons:

- Daily collection (with review) assures that any communication and/or metering issues are identified within that 24-hour period, thus minimizing the loss of data beyond that window.

- A daily data window is a convenient to perform various processing and benchmarking routines for which energy/facility managers can review and make decisions. For hourly data retrieval (recommended where possible), these data can be aggregated-up to present the daily statistics and benchmarks.

- Data quality checks (data receipt, within range, and complete) are easily implemented within the daily window. For systems reporting hourly data, it is recommended that these checks be implemented at the reporting interval (i.e., hourly).

Chapter 6 provides details on both traditional and modern communications and storage methods.

\subsubsection{Data Loss}

Whether by communications issues, metering equipment malfunction, or data processing error, data loss is an inevitable outcome for any reporting system. The first objective upon recognition of data loss is to 
understand and correct the cause. This may involve staff from a variety of trades and expertise from software/IT to electricians. Typical causes of data loss include:

- Data transfer interruption. Lapses in data transmissions can emanate from a number of causes and will depend on the mode of data transmission. Common causes are:

- Loss of power to building/meters, communications nodes, or data processing end-point

- Loss of phone connection (service loss, advertent/inadvertent disconnect)

- $\quad$ RF interference (numerous potential sources) on wireless transmissions

- Data corruption. While less common than interruption, data corruption is also mode dependent. Corruption can be manifest as no data, partial data, or irrational/suspicious data. Causes of corrupt data can be metering/sensor errors, communications interference (noise on lines or RF), or data receiving errors.

- Data interception. In today's world of encrypted data transfer, the concept of successful malicious data interception of standard building energy-use information is improbable. However, acknowledging best practices for data transfer security, it is recommended that all transfers be encrypted and system "hardness" is commensurate with perceived value and uses of these data. Section 3.7.3 discusses standard practice for secure data transfer.

To mitigate the impacts of data loss, it is recommended that all automated data posting systems and/or database processing functions be alarmed to identify lapses or out-of-range values. These alarms should continuously operate and be forwarded to relevant parties (email, text, pager) and/or highlighted on system dashboards.

Once identified and corrected, the focus shifts to understanding the magnitude of data loss and any corrective action necessary to the data set. In general, there are two methods (ASHRAE 2002) to handle missing data:

- Substitution. This method requires additional information or data specific to the lost points. Missing data can be substituted with rational fixed values, calculated average values, or values interpolated from known points.

- Omission. If missing data is more significant than substitution procedures will reasonably accommodate, these points and the resulting analysis can be omitted. While data gaps can be challenging, large data-set substitution may introduce unintended biases and other inaccuracies far more problematic than the missing data.

Regardless of the method chosen, it is important to document any data gaps and the corrective action taken.

\subsubsection{Project Tracking}

Section 432 of the Energy Independence and Security Act of 2007 (EISA 2007) established a framework for facility project management and benchmarking. Per EISA 2007, agencies must identify all "covered facilities" that constitute at least 75 percent of the agency's facility energy use. An energy manager must be designated for each of these covered facilities. In addition to other activities, the facility energy manager will be responsible for: 
- Using a Web application from DOE to certify and track compliance for energy and water evaluations, project implementation and follow-up measures, and estimated cost and savings. The Web application will be available to Congress, other Federal agencies, and the public, with some specific data exempted from disclosure for national security purposes.

- Entering energy-use data for each metered building into a benchmarking system, such as the ENERGY STAR ${ }^{\circledR}$ Portfolio Manager. DOE issued Building Energy Use Benchmarking Guidance on April 15, 2010, that specified Portfolio Manager as the building energy use benchmarking system (FEMP 2010b). Currently, some building types are not considered in Portfolio Manager and agencies shall use alternative benchmarking systems. Tools that can be used for these building types are listed in Section 4.2.5.6.

The Federal Energy Management Program (FEMP) is responsible for tracking Federal agency progress toward meeting EISA 2007 Section 432 for Federal facility energy and water management and benchmarking. To meet this need, FEMP has developed the EISA 2007432 Compliance Tracking System (CTS), which tracks agency performance of energy and water evaluations, project implementation and follow-up measures, and annual building benchmarking requirements.

The EISA 2007432 CTS accepts required Federal agency reporting on:

- Designation and location of facilities covered by EISA 2007 requirements

- Assignment of energy managers

- Energy and water evaluations completed at covered facilities

- Implementation of energy and water efficiency measures (ECMs), including estimated cost and savings

- Follow-up status on implemented measures, including measured savings and persistence of savings

- Building benchmarking information.

Further guidance on the CTS, links to public progress reports, and access for Federal agency users can be found at http://www1.eere.energy.gov/femp/regulations/facility_cts.html.

\subsubsection{Data Security}

The fundamental question that should be answered prior to any decisions on data security is: Are these data sensitive? Asked differently: What are the potential impacts if these data are compromised? The responses to these questions, and level of sensitivity/impact identified, will serve as a gage to overall data security needs.

Metered data security is currently being driven by the proliferation of smart meters and developments with the smart grid. Building off the data security and transfer protocols developed for financial and other internet transactions, metered data security is best implemented at the point of data collection (i.e., at the meter).

While there are a number of evolving data encryption protocols and technologies for secure data transfer such as Public Key Infrastructure (PKI) which were developed to both protect data and authenticate the sender, this field is largely vendor driven. The National Institute of Standards and Technology (NIST) 
Computer Security Division has developed a special publication series (NIST 2011) focused on information technology security and its collaborative activities with industry, government, and academic organizations.

To help evaluate metering equipment security offerings, consider asking the following questions of prospective vendors:

- How does the meter handle data encryption? What technologies/protocols are used?

- What security features are integral to the meter versus software add-ons?

- How is data security handled between auxiliary components such as radios, routers, and collectors?

- What happens in the event of a security breach?

- How are security upgrades handled?

- How is your system certified?

Ultimately, data security will be a function of the meter and communications system selected. Site IT staff need to be part of this decision to assure any solution is appropriate, relevant, and compatible with existing IT security systems.

\subsection{References}

Architectural Energy Corporation (AEC). 2003. Advanced Utility Metering. Under contract NREL/SR-710-33539, Boulder, Colorado.

American Society of Heating, Refrigeration, and Air-Conditioning Engineers (ASHRAE). 2002. Guideline 14-2002: Measurement of Energy and Demand Savings. Atlanta, Georgia.

Better Bricks. 2011. The High Performance Portfolio: Energy Tracking and Accounting. Better Bricks - Bottom Line Thinking on Energy. Available at: http://www.betterbricks.com.

Energy Independence and Security Act of 2007 (EISA 2007). 2007. Public Law 110-140. Signed December 19, 2007.

Energy Policy Act of 2005 (EPAct 2005). 2005. Public Law 109-58, as amended, Section 1252, Smart Metering.

Executive Order (EO) 13423. 2007. Strengthening Federal Environmental, Energy, and Transportation Management. Signed January 24, 2007.

Executive Order (EO) 13514. 2009. Federal Leadership in Environmental, Energy, and Economic Performance. Signed October 8, 2009.

Federal Real Property Statistics (FRPS). 2010. FY 2009 Federal Real Property Report. The Federal Real Property Council. Available at: http://www.gsa.gov/graphics/ogp/FY2009_FRPR.pdf. 
Federal Energy Management Program (FEMP). 2007. FEMP Metering Training Course Session 3: Metering Planning, Financing, Uses for Data and Case Studies. April 4, 2007. Available at: http://eere.pnnl.gov/femp/metering_webcast.stm.

Federal Energy Management Program (FEMP). 2010a. Operations \& Maintenance Best Practices: A Guide to Achieving Operational Efficiency, Release 3.0, prepared by Pacific Northwest National Laboratory for the Federal Energy Management Program, U.S. Department of Energy, Washington, D.C. Available at: http://www1.eere.energy.gov/femp/pdfs/omguide_complete.pdf.

Federal Energy Management Program (FEMP). 2010b. Building Energy Use Benchmarking Guidance: EISA Section 432 - Benchmarking Federal Facilities. U.S. Department of Energy, Energy Efficiency and Renewable Energy, Washington, D.C. Available at: http://www1.eere.energy.gov/femp/regulations/guidance.html.

Heller J. 2005. Energy 2005 Effective O\&M Solutions: Draft Metering Policy. Presentation at GovEnergy 2005.

Lewis J. 2010. Advanced Metering Workshop. Presented at the FEMP Forum on Advanced Metering Solutions for Federal Agencies. Available at: http://www1.eere.energy.gov/femp/pdfs/ns/advmet_12072011_lewis.pdf.

National Institute of Standards and Technology (NIST). 2011. Special Publications Series 800. NIST Computer Security Division, Computer Security Resource Center. Gaithersburg, Maryland.

Pacific Northwest National Laboratory (PNNL). 2010. Guidelines for Estimating Unmetered Industrial Water Use. PNNL-19730. Available at: http://www.pnl.gov/main/publications/external/technical_reports/PNNL-19730.pdf.

U.S. Department of Defense (DoD). 2009. Department of Defense Instruction 4170.11, Installation Energy Management. Available at: http://www.dtic.mil/whs/directives/corres/pdf/417011p.pdf.

U.S. Department of Energy (DOE). 2006. Guidance for Electric Metering in Federal Buildings. DOE/EE-0312. Available at: http://www1.eere.energy.gov/femp/pdfs/adv_metering.pdf.

U.S. Department of Energy (DOE). 2010. Guidelines for Estimating Unmetered Landscaping Water Use. PNNL-19498. Available at:

http://www1.eere.energy.gov/femp/pdfs/est_unmetered_landscape_wtr.pdf.

\section{Websites:}

DOE-2: http://gundog.lbl.gov/dirsoft/d2whatis.html

EnergyPlus: http://apps1.eere.energy.gov/buildings/energyplus

Facility Energy Decision System (FEDS): http://www.pnl.gov/feds/ 


\section{Chapter 4 Metering Approaches}

\subsection{Introduction}

Metering provides the information that when analyzed allows the building operations staff to make informed decisions on how to best operate mechanical/electrical, gas, and steam systems and equipment. These decisions will ultimately affect energy costs, equipment costs, and overall building performance. Metering can take place at a variety of points within an electrical or mechanical system and can encompass the collection of electricity, natural gas, water, steam or other fluid data. The decision of where and what to meter is determined by your metering objectives and should be a focus of your metering plan. While metering at the end-use or circuit level has application and is described in this chapter, the primary focus is on higher-level whole-building utility metering.

At the outset, it should be noted that metering in and of itself saves no energy or dollars. In fact, it cost money to meter, including the purchase and installation of the metering, the communications or meterreading expense, and the time necessary to process and interpret data. A metering program can be a costly and time-consuming endeavor. The key to a successful metering program lies in the ability to make use of the output of a meter. Metered data need to be converted to information from where actions and projects are developed and implemented.

\subsection{Generic Approaches}

The four predominant levels of resource metering (EPRI 1996) are:

- One-time/spot measurement (system/sub-system)

- Run-time measurement (system/sub-system)

- Short-term monitoring (system/sub-system/whole building)

- Long-term monitoring (system/whole building)

The decision of
where and what to
meter is determined
by your metering
objectives and
should be a focus of
your metering plan.

Each level has its own unique characteristics - no one monitoring approach is appropriate for all metering activities. A short description of each monitoring level is provided below. It should be noted that while the four levels of resource metering presented can be applicable to measurement and verification $(\mathrm{M} \& \mathrm{~V})$ of energy projects, the authors of this guide recommend the current U.S. Department of Energy Guidelines for Federal projects (DOE 2008) as a particularly good reference for M\&V protocols.

\subsubsection{One-Time/Spot Measurements}

One-time measurements are useful in many "baseline” activities to understand instantaneous energy use, equipment performance, or loading. These measurements become particularly useful in trending equipment performance over time. For example, a spot measurement of a boiler-stack exhaust temperature, taken periodically (e.g., monthly) and trended over time, can be very diagnostic of boiler efficiency. 


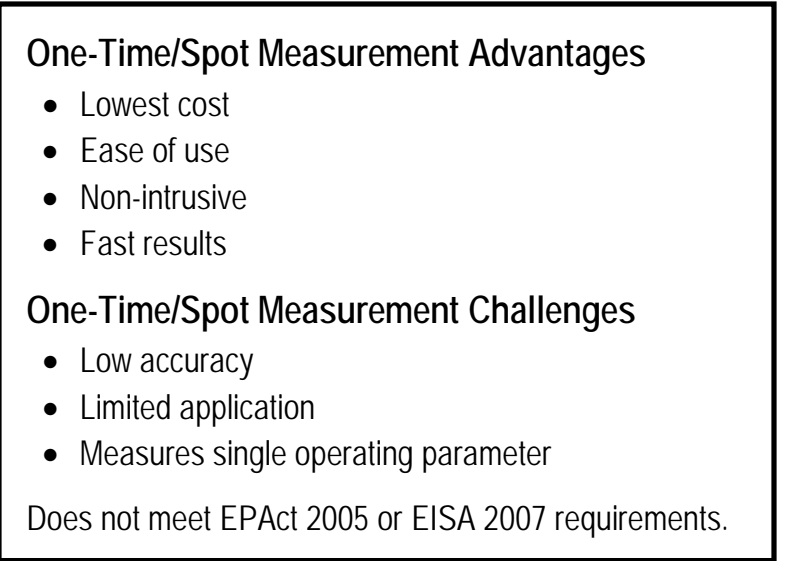

\subsubsection{Run-Time Measurements}

Run-time measurements are made in situations where hours-of-operation are the critical variable. These measurements are prevalent where an energy efficiency project has impacted the use (i.e., hours of operation) of a device. Appropriate applications for run-time measurements include the run times of fans and pumps, or the operational characteristics of heating, cooling, or lighting systems.

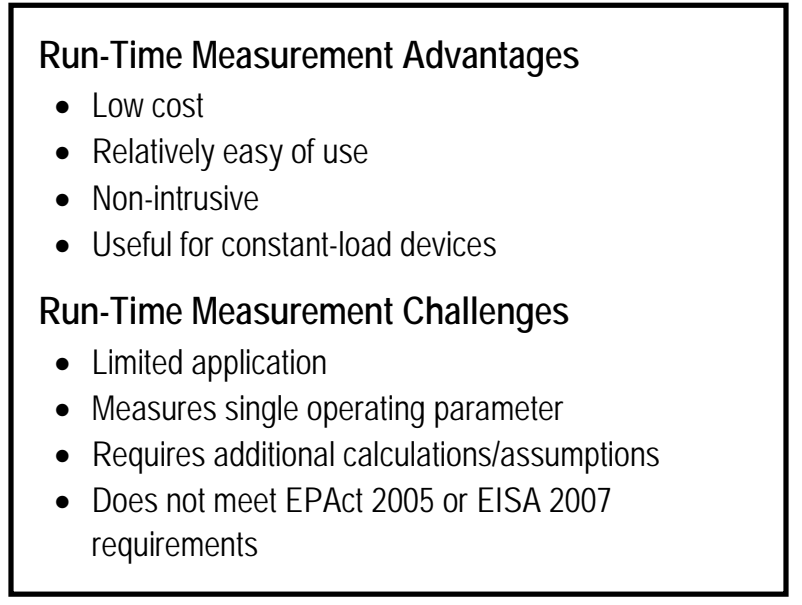

Because run-time measurements do not capture the energy-use component of the system, these measurements are typically used in conjunction with one-time/spot measurements. Equipment useful in making run-time measurements include a variety of stand-alone (battery-operated) data loggers providing a time-series record of run-time. Most of these devices are non-intrusive (i.e., the process or system is not impacted by their use or set-up) and are either optically triggered or take advantage of the electromagnetic characteristics of electrical devices. Run-time measurements are usually obtained in the field by the device, recorded to memory, and then downloaded by the analyst at a later date.

\subsubsection{Short-Term Measurements/Monitoring}

Short-term monitoring combines both elements of the previous two levels into a time-series record of energy or resource use: magnitude and duration. Typically, short-term monitoring is used to verify performance, initiate trending, or validate energy efficiency improvement. In this level, the term of the monitoring is usually less than one year, and in most cases on the order of weeks to months. In the case of energy efficiency improvement validation, also known as measurement and verification, these measurements may be made for 2 weeks prior and post installation of an efficiency improvement project. 
These data are then, using appropriate engineering and statistical methods, extrapolated over the year to report the annual impact.

Equipment useful in short-term monitoring includes a host of portable, stand-alone data loggers capable of multivariate time-series data collection and storage. Most of these data loggers accept a host of sensors including temperature, pressure, voltage, current, etc., and have standardized on input communications (e.g., 4 to 20 milliamps or 0 to 5 volts). These loggers are capable of recording at user-selected intervals from fractions of a second, to hourly, to daily recordings. These systems usually rely on in-field manual downloading or, if available, modem and/or network connections.

\subsubsection{Long-Term Measurements/Monitoring}

Long-term monitoring also makes use of time-series recording of energy or resource use, but over a longer duration. Different from short-term use, this level focuses on measurements used in long-term trending or performance verification. The term is typically more than a year and quite often the installation is permanent. Metering at the whole-building level is typically a long-term, permanent installation metering. This level of metering is the target of the Energy Use Measurement and Accountability portion of the Energy Policy Act of 2005 (EPAct 2005) and Section 434(b) of the Energy Independence and Savings Act of 2007 (EISA 2007).

Useful applications for this level of monitoring include situations where system use is influenced by variances in weather, occupant behavior, or other operating conditions. Other applications include reimbursable resource allocation, tenant billing activities, or in cases where the persistence of energy or resource savings over time is at issue. Other potential benefits include evaluating the persistence of savings over time and ability to benchmark the resource use of the building over time.

Short-Term Measurement Advantages

- Mid-level cost

- Can quantify magnitude and duration

- Relatively fast results

Short-Term Measurement Challenges

- Mid-level accuracy

- Limited application

- Seasonal or occupancy variance deficient

- More difficult to install/monitor

- Does not meet EPAct 2005 or EISA 2007 requirements

Equipment useful in long-term monitoring included a variety of data loggers, utility-grade meters, or fixed data acquisition systems. In most cases, these systems communicate via a network connection or a phone modem to a host computer and/or over the Internet.

\subsubsection{Benchmarking}

As defined for the Federal sector (EISA 2007), benchmarking is the process of accounting for and comparing a metered building's current energy performance with its energy baseline, and/or comparing 
this with the energy performance of similar types of buildings (based on use, such as comparing the performance of an office building to that of other office buildings). Benchmarking can be used to compare performance over time, within and between peer groups, or to document top performers.

As stated in EISA 2007 Section 432 Paragraph A:

The energy manager shall enter energy use data for each metered building that is (or is a part of) a facility that meets the criteria established by the Secretary under paragraph (2)(B) into a building energy use benchmarking system, such as the Energy Star Portfolio Manager.

Each energy manager shall post the information entered into, or generated by, a benchmarking system under this subsections, on the web-based tracking system under paragraph (7)(B). The energy manager shall update such information each year, and shall include in such reporting previous years' information to allow changes in building performance to be tracked over time."

Given these two requisites for use of metered data in a benchmarking construct, it becomes important to understand both the process and the application to meet this legislation. To begin, some definitions:

Benchmarking System - A tool or system of tools that enables the energy performance of a metered building to be compared with itself or other similar buildings. These tools can be as simple as a buildingspecific spreadsheet or as sophisticated as commercial software capable of normalization and regression techniques.

Benchmark Metrics - Values most commonly developed and tracked include electricity use (e.g., kWh per square feet), natural gas (e.g., therms per square feet), and steam (e.g., MBtu per square feet); water use is becoming a common benchmark metric.

Energy Baseline - An initial period of metered energy consumption used as a point of reference for comparison purposes. Most benchmarking analyses make use of a 12-month baseline period to attempt to capture all seasonal aspects of energy use.

Energy Rating - The relative indicator of performance obtained from a benchmarking analysis. This rating can be in engineering units or a scale-based system. In either case, the rating allows the energy use of the metered building to be compared over time with itself and with the energy use of similar buildings.

Metered Building - A facility with one or more meters (advanced or standard) installed to measure energy used within that facility.

Normalization - In comparing benchmarked buildings, it is often the case that other influencing factors need to be taken into account for a fair comparison. Examples of these factors include variations in climatologic data or occupancy patterns. To account for these differences, some benchmarking tools have developed routines that allow the user to enter values to account for these variances.

Web-based Tracking System - A system used in the Federal sector for reporting and updating all benchmarked buildings. 


\subsubsection{Data Required for Benchmarking Compliance}

The following data is necessary in order to meet the EISA 2007 benchmarking requirements and to ensure that the designated energy managers have the information they need so to fulfill the requirements of EISA 2007 Section 432.

- Building Characteristics:

- Type of building or facility (according to use, such as office, hospital, courthouse, warehouse, etc.)

- Building or facility location - depending on the benchmarking tool, this may be the climate zone or zip code

- All floor area (gross square feet) in the building.

- Energy Consumption:

- Monthly or annual (depending on the benchmarking system) site energy consumed by the building and measured using standard or advanced meters.

- To obtain a complete and accurate benchmarking result, all sources of energy consumed by a building will need to be entered into the benchmarking system.

\subsubsection{Benchmarking Goal}

The aim of agency benchmarking should be to compare a building's performance to similar buildings or to the building's prior year performance. Many buildings have different energy-using space types within the building, such as laboratory space and data center space within an office building. These space types within the building should be indicated along with the correlating percent floor space of each.

\subsubsection{Benchmarking Systems}

DOE has selected the ENERGY STAR Portfolio Manager Tool (Portfolio Manager) as the building energy use benchmarking system required for the building types discussed under this subheading (see EISA 2007, Section 432, 42 USC 8253(f)(8)). Some of Portfolio Manager's characteristics include:

- All commercial and institutional buildings can use Portfolio Manager to track energy consumption over time, and also can track water consumption, energy costs, water costs, and carbon emissions

- Offering a comparison of the energy performance of a building to a statistically representative model created with data from DOE’s Commercial Building Energy Consumption Survey (CBECS)

- Providing energy performance ratings for several commercial building types that represent over 60 percent of the U.S. commercial floor space

- Portfolio Manager is a Web-based application with secure access.

Portfolio Manager rates the energy consumed by a building or facility on a scale of 1 to 100 , relative to similar buildings nationwide. Known as the ENERGY STAR Energy Performance Rating, this score is based on comparing 12 months of weather-normalized energy data for each meter in the facility to a 12-month energy baseline. It also adjusts for the unique operating characteristics of the building, for example operating hours or occupancy. The generated score indicates a percentile rank of the facility 
once all required data is entered. A score of 75 or higher qualifies the building to earn the ENERGY STAR Building Label. While attainment of the ENERGY STAR Building Label is encouraged, it is not required.

Building space types eligible to receive an Energy Performance Rating using Portfolio Manager include:

- Offices

- Medical office buildings

- Hospitals

- Courthouses

- Warehouses

- Residence halls/dormitories

- Banks/financial institutions

- Hotels

- K-12 schools

- Houses of worship

- Retail stores

- Supermarkets

- Wastewater treatment plants.

Portfolio Manager can track the energy-use intensity of a campus or other collection of buildings at the same geographic location. However, the campus as a whole cannot receive an Energy Performance Rating. The only exception to this is hospital campuses, which must include support facilities if they are not part of the main hospital building.

Benchmarking of laboratories and data centers is not covered by the Portfolio Manager tool. However, other tools are available to address these applications. Specific information on these tools is provided in Section 4.2.5.6.

\subsubsection{Normalization}

Normalization is the process of recalculating energy use metrics to account for dissimilar variables between comparison buildings. This is a critical step in the benchmarking process as it allows for adjustment for differences in building energy use based on "other" influencing factors. These factors may include:

- Variance in climatologic data

- Differences in operating schedules

- Building occupancy 
- Differences in use-type within a building

- Variances in loads (e.g., a process load within a building).

To account for these variables, some benchmarking tools have developed routines that allow the user to enter values to account for these differences.

\subsubsection{Benchmarking Applications}

The value of any benchmarking system lies in the commitment to its use (i.e., data population and updates) and follow-through with action. The most common applications of benchmarked data are:

- Building comparisons - through system output metrics building assessments and comparisons are easily made. Some of the more common metrics are:

- Energy-use intensity given as monthly or annual energy use per unit area

- Energy score - a derivative of normalized energy-use intensity

- Goal progress - by trending benchmarked metrics organizations can tract (and report) progress to efficiency goals. These tools can represent a repository for data and provide a location for required central reporting.

- Identifying inefficiencies - once populated, benchmarking analysis can be a very powerful tool to highlight and direct identification of efficiency opportunities. If benchmarking takes place on a monthly basis - a normalized energy benchmark should not see significant swing. It there are significant changes, further investigation should be initiated.

\subsubsection{Available Software Tools}

\section{Portfolio Manager}

Description: Portfolio Manager is an interactive energy management tool that allows the tracking and assessment of energy and water consumption across an entire portfolio of buildings in a secure online environment. Whether the entered buildings are owned, leased, or managed for investment, Portfolio Manager can help set investment priorities, identify under-performing buildings, verify efficiency improvements, and receive EPA recognition for superior energy performance.

Availability: http://www.energystar.gov/index.cfm?c=evaluate_performance.bus_portfoliomanager

\section{Labs 21 Benchmarking Tool}

Description: The Labs21 Energy Benchmarking Tool is a Web-based database tool that contains energyuse information from more than 200 laboratory facilities. It allows users to benchmark energy performance in terms of whole-building metrics (e.g., Btu/sf-yr) as well as system-level metrics (e.g., ventilation $\mathrm{W} / \mathrm{cfm})$.

Availability: http://www.epa.gov/lab21gov/toolkit/benchmarking.htm 


\section{Data Centers}

Description: The Data Center Energy Profiler, or DC Pro, is an online software tool to help industries worldwide identify how energy is being purchased and consumed by their data center(s) and also identify potential energy and cost savings. DC Pro is designed so that the user can complete a data center profile in about an hour. When you complete a DC Pro case you are provided with a customized, printable report that shows the details of energy purchases for your data center, how energy is consumed by your data center, potential cost and energy savings, comparison of your data center energy use versus other data centers, and a list of next steps that you can follow to get you started saving energy.

Availability: http://dcpro.ppc.com/

\subsection{The Metering Hierarchy}

In many cases, the
objective of end-use
monitoring is
equipment
performance,
whether to identify
inefficiency or
validate savings
estimates.

Given the above-described metering approaches, there is a logical order, or hierarchy, to consider as you look to maximize your metering value while minimizing your metering cost. Figure 4.1 presents this concept as a function of level of effort and diagnostic capability. This proposed hierarchy starts at the most aggregate level of data collection and processing - the whole-building meter. Assuming access to interval electric data, this meter and resulting data can be diagnostic in identifying trends and variance in whole-building performance. In addition, these data can be useful in understanding the operation and efficiency of major building systems (e.g., chillers, boilers, air handlers). While the resolution of whole-building data may not be fine enough to identify specific operational or efficiency issues, it can often be used to "frame the question" of what equipment/system is performing inconsistently and in need of further exploration.

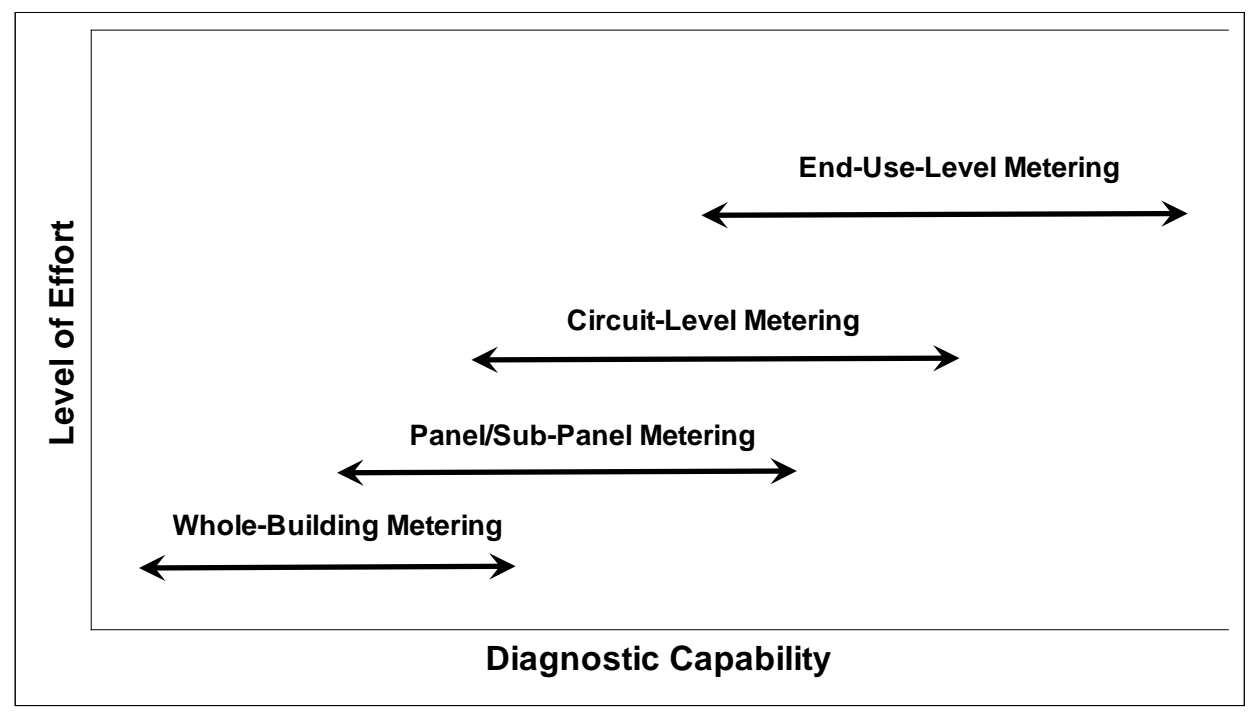

Figure 4.1. Metering Hierarchy 
If the whole-building meter represents the most aggregate level, the next finer level is at the electrical panel/sub-panel. This second tier in the metering hierarchy focuses on loads connected at a panel (or subpanel) level as aggregations of specific loads. Examples of panel-level monitoring include lighting panels or motor panels (i.e., motor control centers - MCCs) where hours of operation or efficiency project validation are of interest. Metering at this level incorporates a variety of dedicated or portable metering equipment or data loggers. The report titled Portable Data Loggers Diagnostic Tools for EnergyEfficient Building Operations (PECI 1999), does a particularly good job of describing some of the equipment useful for metering at this and the following levels.

Moving down one more level in the hierarchy, we examine circuit-level monitoring. The focus of metering at this level is within the panel or sub-panel and the monitoring of a specific circuit of interest. This circuit may have specific plug loads of interest such as computers or other peripherals, or may be of interest for power quality or harmonics studies.

The final level in the hierarchy, having the finest data resolution, is the end-use level. End-use monitoring serves to isolate a particular system or equipment type for detailed study. In many cases, the objective of end-use monitoring is equipment performance, whether to identify inefficiency or validate savings estimates. Chillers, boilers, cooling towers, pumps and motors are often enduse metered for performance metrics.

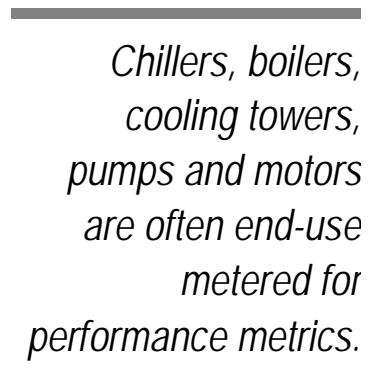

While the above hierarchy presents a step-wise approach to metering and efficiency diagnostics, by no means are we suggesting that all hierarchy steps need be followed sequentially when moving from wholebuilding to end-use metering. In fact, in some cases there should be enough information to move from the whole-building level directly to end-use level when diagnosing or trending efficiency opportunities. In cases where inefficiency by specific equipment is not so apparent, the additional steps may be beneficial to properly identify the poorly operating equipment.

Metering of gas and steam consumption represents a portion of the energy use in one or more buildings typically supporting process-related activities in that building. Sites with multiple process applications in distributed buildings may require the use of multiple gas or steam meters to gain an understanding of potential efficiency opportunities. That will be dependent on the relative energy use for any specific process activity or the location of the use on the site. Depending on the process or amount of gas or steam use, the process might already include metering information regarding consumption. If that situation exists, there is the potential to acquire that data through a central collection point without the installation of a building- specific meter.

\subsection{References}

Energy Independence and Security Act (EISA 2007). 2007. Public Law 110-140. Signed December 19, 2007.

Energy Policy Act of 2005 (EPAct 2005). 2005. Public Law 109-58, as amended, Section 103, Energy Use Measurement and Accountability, Section 543 (42 USC 8253), (e) Metering of Energy Use. 
Electric Power Research Institute (EPRI). 1996. End-Use Performance Monitoring Handbook. EPRI TR-106960, Palo Alto, California.

Portland Energy Conservation, Incorporated (PECI). 1999. Portable Data Loggers Diagnostic Tools for Energy-Efficient Building Operations. Prepared for the U.S. Environmental Protection Agency and U.S. Department of Energy by Portland Energy Conservation, Incorporated, Portland, Oregon.

U.S. Department of Energy (DOE). 2008. M\&V Guidelines: Measurement and Verification for Federal Energy Projects - Version 3.0. Energy Efficiency and Renewable Energy, Washington, D.C. Available at: http://www1.eere.energy.gov/femp/pdfs/mv_guidelines.pdf. 


\section{Chapter 5 Metering Technologies}

\subsection{Introduction}

At the most basic level, all meters track and provide some output related to resource use - energy, water, natural gas, etc. Beyond this basic level, more sophisticated meters take advantage of additional capabilities including electrical demand tracking, power quality measurements, and multiple-meter communication for leak detection applications. For electrical systems, meters can be installed to track whole-building energy use (e.g., utility meters), sub-panel energy use (e.g., a lighting or process circuit), or a specific end use (e.g., a motor or a chiller). An increasingly useful electrical meter type is known generically as an advanced interval meter. These meters measure electrical demand (kW) over a predetermined interval - commonly every 15 minutes - to match utility billing intervals. Other intervals (e.g., 1 minute, 5 minute, hourly) can be useful for examining equipment performance, trending, and start/stop characteristics.

For water, natural gas, and other flow-related applications, meters are typically in-line installations using positive displacement, insertion turbine, or pressurerelated techniques. Depending on the need, any of these meters will vary in size, type, output configuration, accuracy, and price. As with electrical metering, fluid meters should have digital-output capability to take advantage of remote or automated meter reading. The interval metering concept is not exclusive to electricity metering, natural gas, water, and steam meters can also provide these often beneficial data.

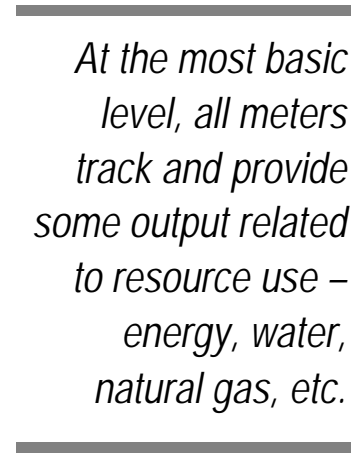

Common to most meters are rated levels of performance; some of the more universal performance metrics are listed below.

Accuracy - this is usually the first metric used to determine applicability of a meter to a particular system. No meter is 100-percent accurate and most manufacturers provide a range of accuracies in their product line and corresponding prices. Accuracy could be thought of as the difference between a measured value and that of the actual value. Published accuracies often will, and should, be referenced to specific calibration procedures including equipment-traceability to National Institute of Standards and Technology (NIST 2010) equipment and procedures.

Precision/Repeatability - the precision or repeatability of a measurement entails the ability to reproduce the same value (e.g., temperature, power, flow rate) with multiple measurements of the same parameter, under the same conditions.

Turndown ratio - the turndown ratio refers to the flow rates over which a meter will maintain a certain accuracy and repeatability. For example, a steam flow meter that can measure accurately from $1,000 \mathrm{lbs} / \mathrm{hr}$ to $25,000 \mathrm{lbs} / \mathrm{hr}$ has a turndown ratio of $25: 1$. The larger the turndown ratio, the greater the range over which the meter can measure the parameter.

Metering Best Practices Guide

August 2011 
The lowest cost metering technology may not be the best choice if it has high associated maintenance costs.
Beyond performance metrics, other criteria useful for meter selection include:

Ease of installation - regardless of technology, meters come in many different sizes, shapes, needing a variety of inputs, and offering a variety of outputs. When making specific make-and-model decisions, it is important to understand any size and weight constraints, needs for specific diameters (or lengths) of straight pipe upstream and downstream of the meter, specific electrical and communications needs, and the overall environment the meter will operate in.

Ongoing operations and maintenance - the lowest cost metering technology may not be the best choice if it has high associated maintenance costs (e.g., frequent service, recalibration, sensor replacement). As with most capital purchases, a life-cycle cost approach (including all capital and recurring costs) is recommended for decision making.

Installation versus capital cost - in some situations, the cost to install a meter can be greater than the capital cost; this can be true where system shutdowns are necessary for meter installations, or where significant redesign efforts are needed to accommodate a meter's physical size, weight, or required connection. In these cases, decision makers should consider alternative technologies that may have a high first cost but a much lower installed cost. A good example of this is the use of non-intrusive metering technologies (e.g., ultrasonic flow meters) that typically have a high capital cost but often a significantly reduced installed cost.

equipment selection, compatibility, appropriateness for application, and all operational and maintenance activities.

The next several sections in this guide address metering technologies specific to electricity, natural gas, steam, potable water, and high-temperature/chilled water. Each section was designed to be stand-alone, as such, some of the material may seem redundant; however, the authors felt the need for completeness for the reader who needed information on one technology at the cost of appearing redundant to multiple-technology reader.

Throughout this guide, we will first defer to the manufacturer's recommendations on equipment selection, compatibility, appropriateness for application, and all operational and maintenance activities.

Appendix $\mathrm{C}$ of this guide provides a list of some of the codes and standards that are relevant to metering, metering systems, and communications.

\section{References}

National Institute of Standards and Technology (NIST). 2010. National Institute of Standards and Technology Policy on Traceability. Available at: http://www.nist.gov/traceability/nist_traceability_policy_external.cfm 


\subsection{Electricity Metering}

\subsubsection{Introduction}

The measurement of electricity can be accomplished in many ways, using a variety of instrumentation, with varying degrees of accuracy and reliability. Likewise, the uses for metered electricity data are numerous - ranging from relatively simple totalized monthly kilowatt hours (kWh) to very sophisticated studies of power quality and harmonics. This section discusses the most common electrical metering parameters with the focus on those parameters offering the greatest potential for energy and operational efficiency improvements (FEMP 2007). It should also be restated that electricity metering can be a complicated and dangerous endeavor; the equipment and procedures discussed here should only be attempted by qualified staff and in compliance with the National Electric Code (NFPA.70 2007) and any local- or agency-specific codes or requirements.

\subsubsection{Common Electrical Metering Terms}

Amperes (amps): The measure of electricity flow in a conductor and usually measured with an ammeter or current transformer. The symbol for amperes is “A”.

The uses for metered electricity data are

Voltage (volts): The measure of electric potential between two points in a circuit and typically measured with a voltmeter or potential transformer. The symbol for voltage is "V".

Volt-amperes (volt-amps): The measure of “apparent” rate of energy supplied to an electric load. The volt-ampere (designated VA) is defined as the voltage multiplied by the current. The volt-ampere is the metric used to rate many forms of electrical equipment.

numerous - ranging from relatively simple totalized monthly kilowatt hours ( $k W h$ ) to very sophisticated studies of power quality and harmonics.

Wattage (watts): A measure of the "real power" delivered to an electric load. Watts are defined as volt-amperes multiplied by the "power factor." As such, the real power will always be less than or equal to the apparent power.

Volt-ampere reactive (VAR): A measure of the system's reactive power - or power stored in a system's inductive loads - and is mostly used for identifying power factor correction needs.

Power factor: The ratio of "real power" (watts) to "apparent power" (volt-amperes) and defined as the cosine of the phase angle between voltage and current. For resistive loads (in ac circuits), the voltage and current are in phase and, therefore, the cosine of the angle is unity (i.e., 1.0), resulting in a power factor of unity. For loads with reactive components (e.g., motors, electrical ballasts), the voltage and current are not in phase resulting in a power factor of less than unity. Power factors significantly less than 1.0 (e.g., 0.85) can result in surcharges from the utility due to their need to make up the balance resulting from the improper power factor. The significance of power factor is that the electric utility supplies customers with volt-amps but bills the customer for watts. A power factor below 1.0 requires the utility to generate more than the minimum volt-amps to supply the load. 
Demand: A measure of the average real power over a specified time interval. Depending on the utility, the specified interval is between 5 minutes to 1 hour, with the 15-minute interval being the most common.

Peak demand: The largest value of demand occurring during the billing cycle. This value is typically used by the utility to assess peak demand billing. It is critical to understand how your utility assesses peak demand, and the associated $\mathrm{kW}$ charge, to be able to manage for operational and economic efficiency.

Harmonics: A measure of the electrical frequencies beyond the fundamental frequency of 60 hertz and usually labeled as the first harmonic (60 hertz), second harmonic (120 hertz), and so on. Harmonics are created by non-linear loads (e.g., computer power supplies, electronic ballasts) that draw current in short pulses rather than the traditional smooth ac sine

Mechanical meters

are the most

prevalent of the three

major types of

electricity meters. waveform. Among other problems, harmonics can cause excessive heating of metal wires and certain types of electrical interference.

Total harmonic distortion (THD): THD is a measure of the content of all harmonic frequency current or voltages in relation to the fundamental current or voltage frequency. This content is usually expressed as a percentage of the fundamental frequency and is defined as the square root of the sum of the squares of the harmonics divided by the fundamental frequency.

\subsubsection{Electricity Metering Technologies}

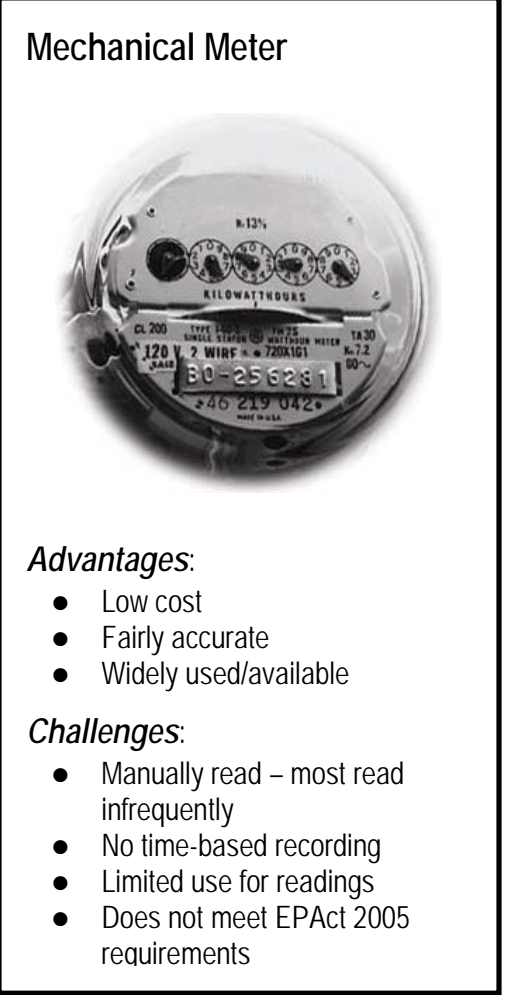

August 2011
There are three major types of electricity meters: (1) mechanical meters where electricity use is proportionate to movement of a mechanical dial, (2) electro-mechanical meters, which are similar to the mechanical meter but with an electronic or pulse output, and (3) solid state/digital meters - also called advanced meters - these meters have no moving parts and outputs reported in a variety of digital formats. A short description of each follows (FEMP 2005):

Mechanical Meters: In terms of installed base, this meter type - due to its extensive use by utilities in the residential sector - is the most mature and has the largest fraction of the installed base. The basic function of this meter involves a metallic disk acted on by two coils; one coil generates a magnetic field proportionate to the voltage and the other a field proportionate to the current. These two fields act on the disk with the force related to the product of voltage times the current. This force causes the disk to spin at a rotational speed proportionate to the power drawn by the load(s) through the meter.

The mechanical meter is commonly found on building service entrances (usually residential and small commercial) with service voltages of 120/240 volts.

When properly installed mechanical meters have accuracy in the range of 1 to 2 percent. Equipment costs for mechanical meters are in 
the range of $\$ 50$ to $\$ 100$. The accompanying installed cost is difficult to estimate without knowing the existing configuration, presence of a meter base, and needs for additional wiring, trenching, etc.

A significant limitation to mechanical meters is the lack of data storage and an ability to electronically communicate. Mechanical meters are in essence accumulators where data are read manually and energy use is calculated as the delta between the current and previous readings. As such, mechanical meters do not meet the EPAct 2005 requirements for interval data (at least hourly) nor an ability to have daily downloads.

Electro-Mechanical Meters: Similar in form and function to the mechanical meter, the electromechanical meter has the same basic operation with the addition of some type of optical encoder for digitizing energy use. This encoder is a device that "sees” the spinning disk and reports a signal (most commonly a pulse) at the completion of one revolution. This pulse can either be stored at the meter for future downloading or sent for collection to an ancillary data recording device. In either case, the electromechanical meter affords the ability to collect, record, and read the electronic representation of power use.

As with the mechanical meter, the electro-mechanical meter is usually found on building service entrances (usually residential and small commercial) with service voltages of $120 / 240$ volts. Because these meters do not usually track demand $(\mathrm{kW})$, their use is limited to smaller utility accounts.

When properly installed, electro-mechanical meters have an accuracy in the range of 1 to 2 percent. Equipment costs for electro-mechanical meters are in the range of $\$ 200$ to $\$ 400$; retrofit costs are in the $\$ 100$ to $\$ 200$ range (NBI 2009). As with all electric meters, the accompanying installed cost is difficult to estimate without knowing the existing configuration, presence of a meter base, needs for additional wiring, and possibility of trenching.

Distinct from the mechanical meter, the electro-mechanical meter does allow for data storage and communication. However, most of these systems are not designed for the interval data storage and communication abilities sufficient to meet EPAct 2005 requirements. While it is possible to configure an electro-mechanical meter to meet EPAct 2005 requirements (at least hourly interval data with daily data access), the ancillary data storage and communication equipment may be cost prohibitive. If this route is being considered for EPAct 2005 compliance, consideration should be given to this ancillary equipment

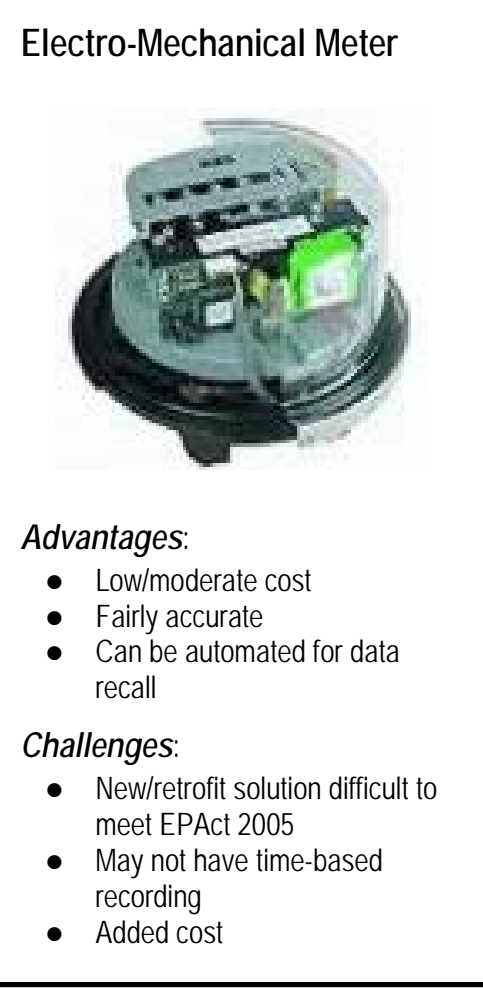
and configuration costs and then compared to a fully capable and integrated advanced electric meter.

Advanced (solid state/digital) Electric Meters: Different from mechanical/electro-mechanical meters, advanced meters require no moving parts, rather they rely on sophisticated integrated circuits with current and voltage transformers, on-board memory, and communication technology. 


\section{Advanced Electric Meter}

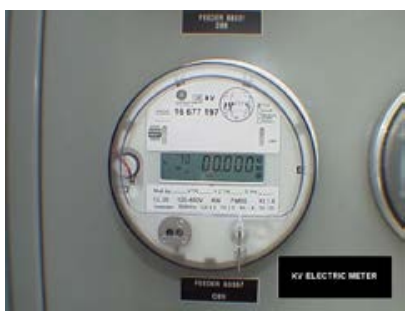

Advantages:

- Accurate

- Data storage and time-stamp capabilities

- Can accommodate other inputs

- Multiple output/diagnostic capabilities

- Two-way communication

- Control/alarm features

- Flexible data intervals and uses

\section{Challenges:}

- Moderate/high cost - More expensive as options and features increase

- More complicated/more data/ staff training suggested

- Need ancillary systems for data transfer and use
Advanced meters are those that have the capability to measure and record interval data (at least hourly for electricity) and communicate the data to a remote location in a format that can be easily integrated into an advanced metering system. These meters measure electrical demand $(\mathrm{kW})$ over a pre-determined interval—commonly every 15 minutes to match utility billing intervals. Other intervals (e.g., 1 minute, 5 minute, hourly) can be accessed and are useful for examining equipment performance, trending, and start/stop characteristics. With availability and versatility of advanced meters increasing and capital costs falling, these meters are quickly gaining market share and acceptance.

Advanced meters are usually installed on larger commercial buildings and/or facilities with large time-varying loads. In these instances, access to energy ( $\mathrm{kWh}$ ) and power $(\mathrm{kW})$ data is critical to understanding energy/power uses and costs. In the Federal sector, it is these meters that meet the full intent of EPAct 2005.

Advanced meters can take a variety of shapes and sizes (also known as the meter "form factor"), from the familiar circular socket-based style to an array of rack and panel-mount configurations. Depending on the manufacturer and model, advanced meters have accuracies in the range of 0.2 to 3.0 percent, with most in the 0.2 to 0.5 percent range. Equipment costs for these meters can also vary by manufacturer and features selected; typical advanced meter cost range from $\$ 400$ to $\$ 700$; meters with advanced diagnostic capability can range as high as $\$ 2,000$ (NBI 2009).

Properly configured, advanced meters meet EPAct 2005 (hourly interval data with daily downloads) and most models go well beyond with advanced features. Some of the more common features of advanced meters are listed below:

- Data storage and time-stamp capabilities - meters can record and store energy, demand, and diagnostics in a time-series record with user-selectable intervals.

- Accommodate other inputs - meters can receive inputs (e.g., pulses from nearby gas or water meters) and record and store these data in the same time series record.

- Diagnostic capabilities - span the electrical horizon from voltage and current diagnostics, harmonic distortion and power factor studies to voltage level and phase symmetry.

- Two-way communications - meters typically have abilities to both send and receive signals which allows remote access to change meter configurations (e.g., data intervals).

- Control/alarm features - as demand $(\mathrm{kW})$ approaches target levels these meters can alarm facility staff of approaching limits and/or control equipment and systems to off-load or shut down. 
- Flexible data intervals - for diagnostic purposes, these meters have flexible recording intervals, usually down to at least 1 minute. This allows facility staff to isolate suspect events in a much refined window.

- Statistics: minimum, maximum, average - meters have extensive statistical capabilities of recording, analyzing, and reporting maximum/minimum voltage and current readings, phase-to-phase relationships, harmonics, etc.

- Multiple modes of communication - most meters have capabilities from traditional phone modem to networked connections and wireless options. In addition, some meters allow for multiple communication options and include an ability to be a communications hub for other devices such as gas or water metering devices.

\subsubsection{Recording Intervals}

For larger commercial buildings and industrial/processing facilities, electric utilities collect, store, and determine billing amounts based on a 15-minute interval. To be able to match utility records, examine load profiles, and understand potential utility rate dollar savings opportunities - using the 15-minute recording interval is recommended.

Beyond the utility-related activities, shorter data intervals offer a host of diagnostic capabilities including identifying peak-demand contributing equipment and opportunities for targeted load shedding/shifting. As mentioned, the ability to vary the recording intervals based on diagnostic or other temporary need can be invaluable; this gives the user the power to examine refined intervals for a period and then change back to the more manageable data intervals.

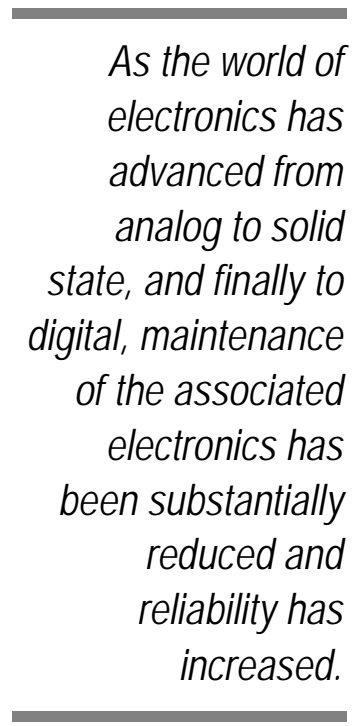

\subsubsection{Electric Metering Maintenance}

As the world of electronics has shifted from analog to solid state, and finally to digital, maintenance of the associated electronics has been substantially reduced and reliability has increased. The electronics associated with power metering are no different than that of most other similar devices. Environmental conditions play a key part in the longevity and reliability of the components. Care should be taken to minimize environmental extremes (e.g., temperature, vibration). Although the metering components can be sent to manufacturers for periodic maintenance and calibration, the functionality of the metering device can also be periodically compared to portable metering devices. Other components of the electrical metering system, for example, current and potential transformers, are generally maintenance free, provided they are originally designed for the operational and environmental conditions. The integrity of electrical connections should be checked periodically in accordance with National Fire Protection Association (NFPA) (NFPA.70 2007) and manufacturer guidance.

\subsubsection{Electric Meter Data Output/Communications Considerations (FEMP 2007)}

For the whole-building application of advanced electric meters, the most common data outputs are calibrated pulses (e.g., pulses/kWh). These data are usually stored at the meter in the prescribed 
time-series format. At periodic intervals (most often daily), the data are accessed through one of a variety of communications options. These data are then downloaded to a database for future processing. Chapter 6 addresses in more detail the different output and communications options.

\subsubsection{Advanced Electric Meter Specification Considerations}

- Determine service voltage - Use design drawings and confirm by system walk-down. If present, understand transformer wiring and output.

- Estimate maximum amperage or power - At the main disconnect is listed the disconnect power as the maximum safe load. The real load is often less

For the wholethan this rating. Also need to confirm there is only one electrical feed to the building application of building and no feeds to other buildings.

advanced electric

meters, the most

common data outputs

are calibrated pulses.

- Physical installation requirements - Where will the meter be installed, internal or external to building? Will installation use a meter base, panel, or rack-mount?

- Determine accuracy requirements - Typical accuracy of advanced electric meters is 0.2 to 0.5 percent.

- Communications requirements - How will the meter report the data: network connection, phone modem, power-line carrier, building automation system?

- Functionality requirements - At the outset, determine the required functionality first and then consider the other options and features.

- Facility staff buy-in - Make certain those staff that will be installing, maintaining, and most importantly, using the data have a voice in meter specification development.

\subsubsection{Advanced Electric Meter Selection Considerations}

- Don’t over-buy - Good metering planning leads to judicious feature selection. Resist the temptation to capture more data than you are able to

When it comes to electric meter selection, resist the temptation to capture more data than you are able to process and use. process and use.

- Consider flexibility - Balance what you need now with that you see as having future benefits. Consider separating "required" and "desired" features. Understand if features can be enabled or added later without significant retrofit expense.

- Communication interoperatability - Consider standardization on communication between meters and other data acquisition systems. 
- Data processing - How will the collected data be processed? Does the metering equipment vendor offer this function/service? Do not overlook the effort it will take to create a process to collect, store, and archive the data.

- Facility staff buy-in - Make certain those staff that will be installing, maintaining, and most importantly, using the data have a voice in meter selection.

\subsubsection{References}

Energy Policy Act of 2005 (EPAct 2005). 2005. Public Law 109-58, as amended, Section 103, Energy Use Measurement and Accountability, Section 543 (42 USC 8253), (e) Metering of Energy Use.

Federal Energy Management Program (FEMP). 2005. Facility Metering for Improved Operations, Maintenance, and Efficiency. Federal Energy Management Program (FEMP), O\&M Program Fact Sheet. January 2005. Available at: http://eere.energy.gov/femp/pdfs/om_metering.pdf.

Federal Energy Management Program (FEMP). 2007. FEMP Metering Training Course Session 2: Metering Technologies, Communications, and Data Storage. April 4, 2005. Available at: http://eere.pnnl.gov/femp/metering_webcast.stm.

New Buildings Institute (NBI). 2009. Advanced Metering and Information Systems. Report prepared for the U.S. Environmental Protection Agency.

NFPA.70. 2007. National Fire Protection Association 70, National Electric Code. NFPA, Quincy, Massachusetts. Available at: http://www.nfpa.org/. 
This page intentionally left blank. 


\subsection{Fluid Metering - Natural Gas}

\subsubsection{Introduction}

The metering of natural gas presents unique challenges when compared to other fluid (i.e., liquid) flow metering. The physical properties of natural gas, particularly its dynamic relationship between temperature and pressure, can drive metering inaccuracies unless compensated for. Accurate natural gas flow measurement usually requires the measurement of the fluid's temperature and pressure in addition to flow.

Additional constraints on natural gas metering may include the physical space available or possibly configuration and weight of the metering system. Some of the fluid metering technologies require specific lengths of pipe, both upstream and downstream of the meter for proper function. Before any technology decisions are made, discussions with equipment vendors and/or design engineers are recommended to ensure proper technology selection.

Depending on the application, flow rate, installation access, and desired accuracy, there are a number of technology options for natural gas metering. In general, measurement of natural gas volumetric flow rate is represented in standard cubic feet per minute (scfm). The actual mass of gas flowing past a point of measurement changes with its temperature and pressure. Density changes resulting from temperature and pressure differences result in differences between the energy content of like volumes of the gas. To equalize the effect of density variations when metering gas, conditions are referenced against standard temperature and pressure conditions, hence, scfm instead of cubic feet per minute (cfm). Gas meters must compensate for density differences between standard conditions and actual conditions to accurately define

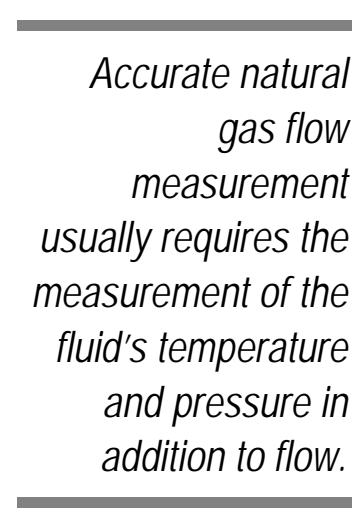
scfm flow rates. The most common volumetric gas metering devices fall into one of the following categories: (1) positive displacement, (2) differential pressure, and (3) velocity. In most applications, gas meters are installed downstream of pressure regulation devices and the meters are then calibrated to that pressure.

As is the rule for all metering, not every natural gas metering technology is recommended for every application. Careful determination of your required accuracy, access, and price point will aid in proper selection.

\subsubsection{Natural Gas Metering Technologies}

As mentioned, there are three primary categories of gas flow measurement relevant to Federal application: positive displacement, differential pressure, and velocity (FEMP 2007). A description of each is provided below.

\subsubsection{Positive Displacement Meters}

As the name implies, a positive displacement meter functions by the fluid physically displacing the measuring mechanism and this displacement becomes the metered value. Of relevancy to natural gas measurement, the two predominant technologies are the diaphragm meter and the rotary meter. In each

Metering Best Practices Guide 
case, the volume of gas for measurement physically impinges on a measuring element (flexible diaphragm or rotary blower) to increment a recording dial or other output.

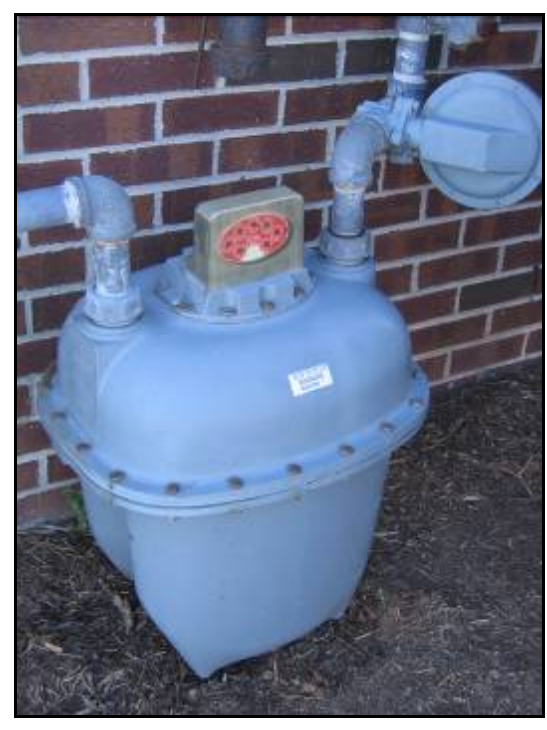

Figure 5.1. Typical Natural Gas Diaphragm Meter
Positive Displacement - Diaphragm Meter. By far the most common type of natural gas meter is the positive displacement “diaphragm” meter (Figure 5.1). Within this meter are usually two or more chambers separated by a moveable diaphragm. As the gas flows into the meter, it is directed by internal valves causing the chambers to alternatively fill and expel the gas. This fill/expel cycle produces near continuous flow of gas through the meter. A rotating crank mechanism converts the linear motion of the diaphragms into rotary motion which then drives an index to record the gas use. The diaphragm meter is commonly found on building service entrances with pipe connection (line) sizes of 0.75 to 2 inches. As with all natural gas metering devices, to ensure the highest accuracy, pressure and temperature compensation should be applied. When properly installed, complete with temperature and pressure compensation, diaphragm meters are accurate typically to within 1 percent. Equipment cost for diaphragm meters is in the range of $\$ 150$ to $\$ 500$ - depending on connection size (NBI 2009). The related installed cost is difficult to estimate without knowledge of existing configuration, the need for piping, trenching/cutting of concrete, or the cost to bring the existing configuration "up to code."

Positive Displacement - Rotary Meter. The other major type of positive displacement meter for natural gas metering is the rotary flow meter. Figure 5.2 shows a typical rotary flow meter.

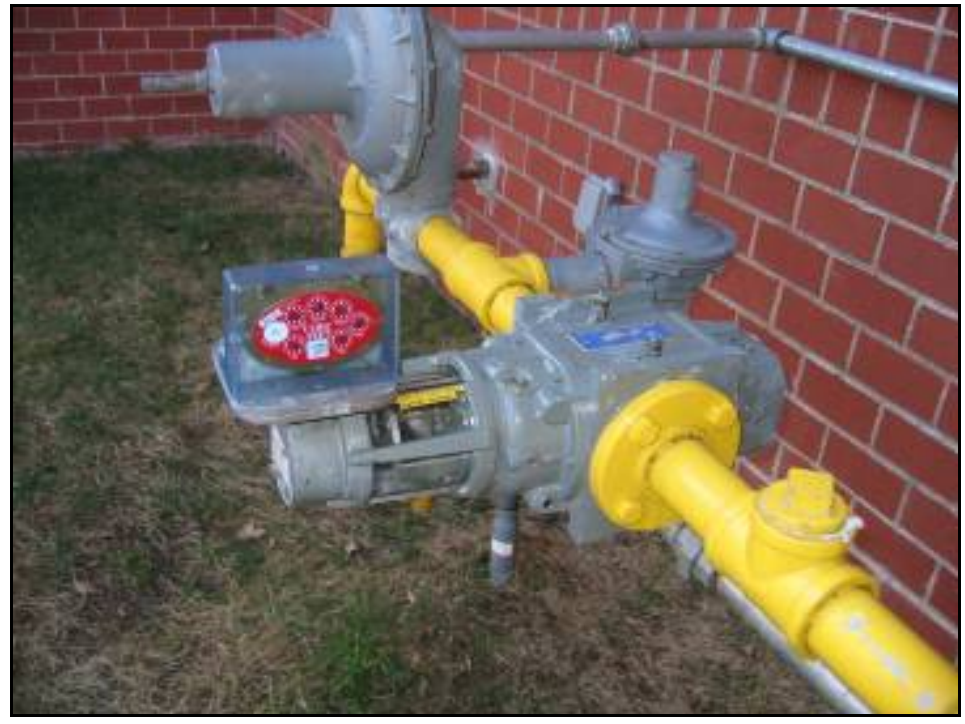

Figure 5.2. Typical Natural Gas Rotary Flow Meter

Rotary flow meters are highly machined-precision instruments capable of handling higher volumes and pressures than diaphragm meters. Their function relies on two figure-eight-shaped lobes that turn, and in 
so doing, trap a specific volume of fluid. As the lobes turn, the fluid is moved through the device with the fluid's flow being proportional to the rotational velocity of the rotors. Figure 5.3 highlights the function of the rotary flow meter.

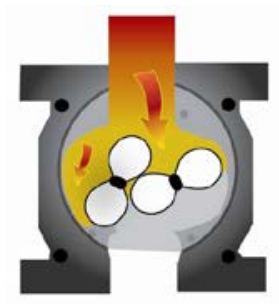

Demand initiates gas flow through the meter.

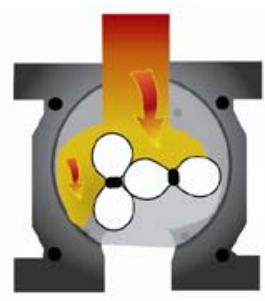

Impeller captures a fixed volume of gas

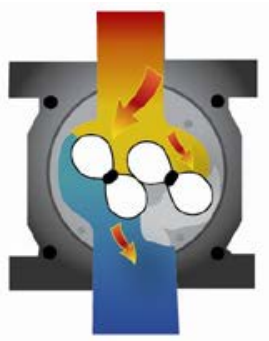

Measuring cavity opens releasing gas downstream.

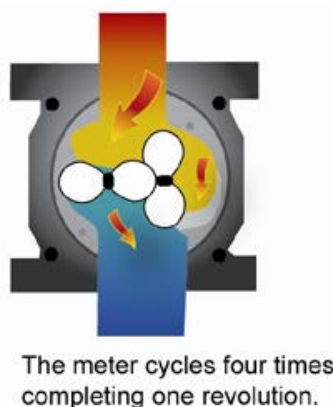

completing one revolution.

Figure 5.3. Rotary Flow Meter Function

The rotary flow meter is commonly found on buildings with line sizes of 1.5 to 4 inches and typically located on buildings with higher flow rates of gas. Rotary flow meters have accuracies in the range of 0.25 to 0.5 percent and, depending on connection size, have costs in the range of $\$ 300$ to $\$ 500$ (NBI 2009).

\subsubsection{Differential Pressure Meters}

All differential pressure meters rely on the velocity-pressure relationship of flowing fluids for operation. Specifically, when an obstruction or orifice is placed in the path of a fluid, the fluid velocity will increase while its pressure decreases. It is this change in pressure that is measured and, via the relation-
The rotary flow meter is commonly found on buildings with line sizes of 1.5
to 4 inches and typically located on buildings with higher flow rates of gas. ship between pressure drop and the square-of-the-flow, used to calculate the fluid flow rate. There are a variety of differential pressure devices useful for gas metering, three of the more common devices are described below.

Differential Pressure - Orifice Meter. Although various-style orifices are manufactured, the basic design and operation remains the same. The orifice element is typically a thin, circular metal disk held between two flanges in the fluid stream. The center of the disk is drilled with a specific-size hole, depending on the expected fluid flow parameters (e.g., pressure and flow range). As the fluid flows through the orifice, the restriction creates a pressure differential upstream and downstream of the orifice proportional to the fluid flow rate. This differential pressure is measured and a flow rate mathematically calculated based on the differential pressure and fluid temperature. Figure 5.4 presents a diagram of a typical orifice meter. 


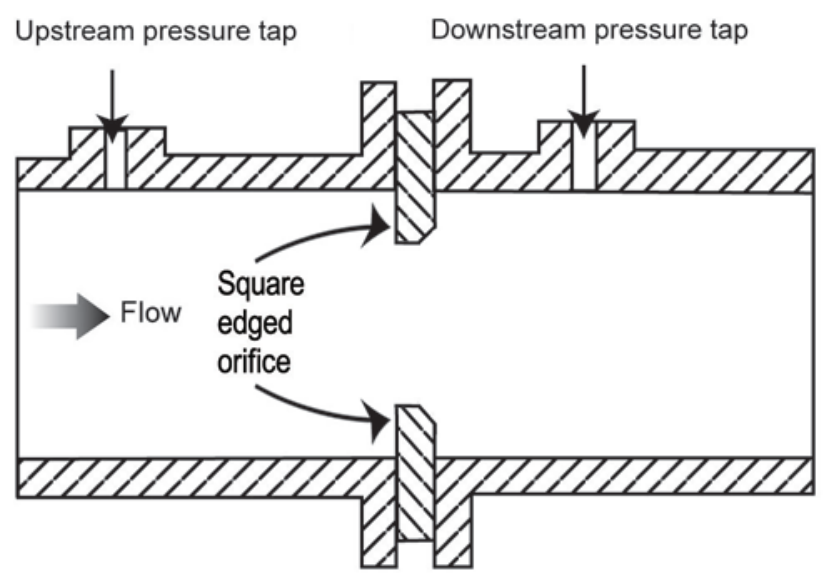

Figure 5.4. Typical Orifice Meter

Orifice meters, by design, develop significant pressure drop within the system. The benefits of this technology (i.e., its relative compactness, its accuracy, and its simple function) should be traded off against the potential decrease in pressure at the end use. For proper function, the orifice meter requires smooth flow through the orifice. For this to be assured, it requires a significant section of straight pipe both upstream and downstream of the meter. While these lengths may vary by manufacturer, typical lengths fall between 15 to 25 pipe diameters upstream and 5 to 15 pipe diameters downstream.

meters should be traded off against the potential decrease in pressure at the end use.
For natural gas metering, orifice meters are used mostly in end-use applications, as opposed to a whole-building metering applications, or where space and equipment size are constraints. Orifice meters are commonly used on connection sizes from 0.25 to 4 inches. These meters have accuracies ranging from 0.25 to 2 percent, depending on the fluid, type of orifice, and installation. Orifice meters range in cost from $\$ 300$ to $\$ 5,000$, with the higher cost systems associated with larger and more accurate meters.

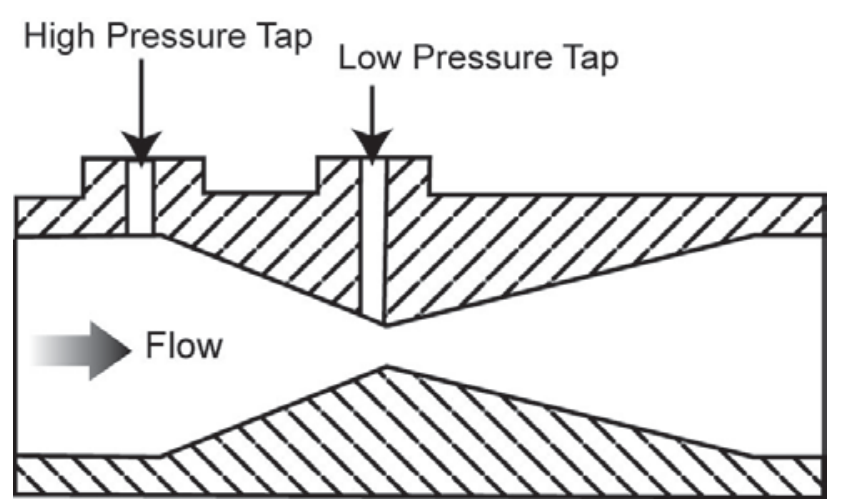

Figure 5.5. Typical Venturi Meter
Differential Pressure - Venturi Meter. Similar in function to the orifice meter, the venturi meter takes advantage of the same velocitypressure relationship (i.e., the change in pressure is proportional to the square of the velocity). In this case, the device causing the change in pressure is not a sharp-edged orifice but rather a section of pipe that gently converges to a small-diameter area (called a throat) before diverging back to the full pipe diameter. Figure 5.5 presents the operation of a typical venturi meter.

The benefit of the venturi meter over the orifice meter lies in the reduced pressure loss experienced by the fluid. In situations where the cost to pump a fluid is high, this benefit can represent a significant savings over the life of the system. An additional benefit of the venturi meter is its durability - it does not have 
the "sharp edge" profile of the orifice meter; therefore, it does not suffer from potential erosion issues and has a greater ability to accurately meter contaminated or non-homogeneous fluids. As with orifice plate meters, venturi meters are used mostly in specialty natural gas applications where size, space, and/or accuracy dictate their use.

Venturi meters can be used on connection sizes ranging from 0.25 to 4 inches and have accuracies in the range of 0.25 to 2.0 percent, depending on fluid type and installation. Venturi meters can cost from $\$ 1,000$ to $\$ 5,000$.

Differential Pressure - Annubar Meter. The annubar flow meter (a variation of the simple pitot tube) also takes advantage of the velocity-pressure relationship of flowing fluids. In this case, the device causing the change in pressure is a pipe inserted into the natural gas flow. Contained within this pipe are two smaller tubes with holes or ports evenly spaced along the length. Figure 5.6 presents the components of the annubar flow meter. When properly installed, one tube faces directly upstream and one downstream. These tubes and ports become the pressure detection points for the meter; the upstream-facing ports measure the flowing pressure (i.e., velocity pressure) and the downstream port measures the static pressure. Using these measured pressure values and the previously mentioned pressure-flow relationship, the flow rate is calculated.

Annubar flow meters have a turndown ratio of up to 10:1 and are relatively easy to install. These meters can make measurements on pipe sizes from 2 to 100 inches to an accuracy of 2 percent. Annubar meters can cost between $\$ 1,000$ to $\$ 3,000$, depending on size and accuracy.

\subsubsection{Velocity Meters}

Velocity meters determine fluid flow by measuring a representation of the flow directly. Because the fluid's velocity is measured (i.e., not the square-root relationship to determine velocity as with differential pressure meters), velocity meters can have better accuracy and usually have better turndown ratios than other meter types.

Velocity - Turbine Meter. Turbine meters operate as their name implies. A multi-blade impellor-like device is located in, and horizontal to, the fluid stream. As the fluid passes through the turbine blades, the impellor rotates at a speed related to the fluid's velocity. Blade speed can be sensed by a number of techniques including magnetic pick-up, mechanical gears, and photocell. The pulses generated as a result of

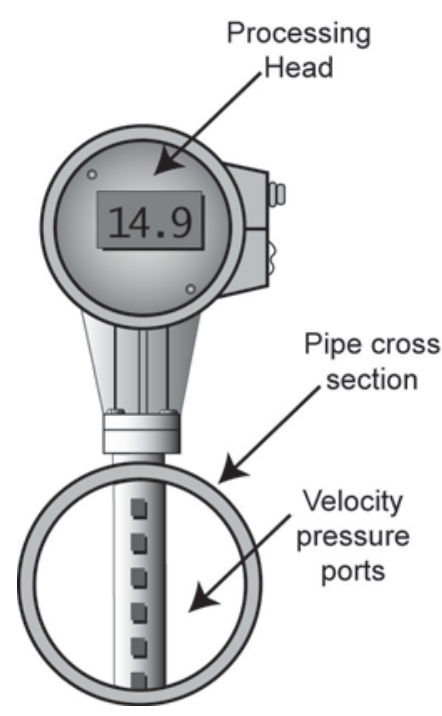

Figure 5.6. Typical Annubar Meter blade rotation are directly proportional to fluid velocity, and hence, flow rate. Figure 5.7 details the components of a typical turbine meter. It should be noted that there are a variety of turbine designs and mounting configurations; Figure 5.7 highlights the concept. 


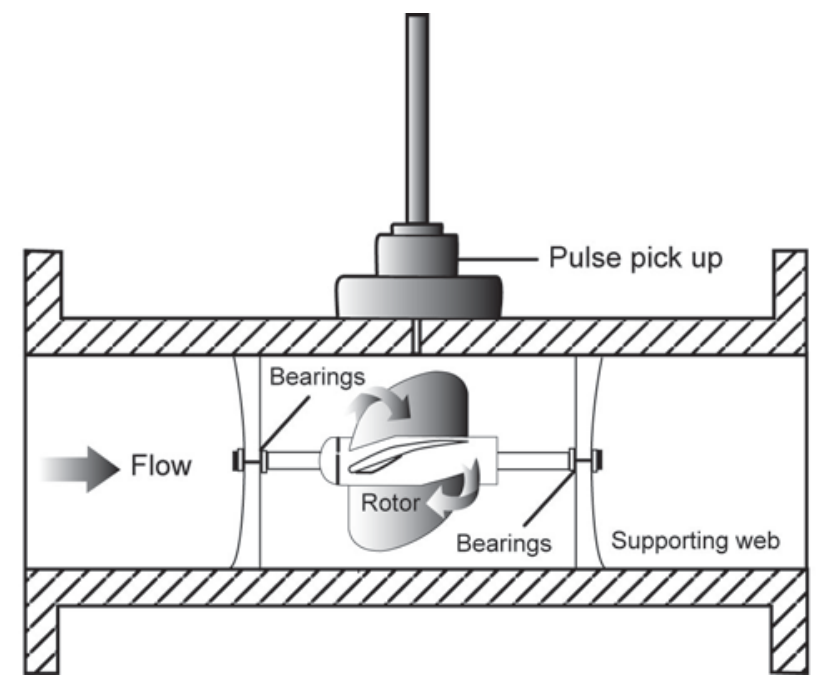

Figure 5.7. Typical Turbine Meter

The natural gas applications for turbine meters are typically for larger industrial metering functions.
Because the turbine meter has mechanical elements in the fluid stream, turbine meters can be susceptible to wear and resulting inaccuracies. Of particular concern are the bearings that, if exposed to corrosive or contaminated fluids, can quickly wear and result in inaccuracy. The natural gas applications for turbine meters are typically for larger industrial metering functions. Turbine meters are used on connection sizes ranging from 2 to 20 inches and have accuracies in the range of 0.5 to 1.0 percent, depending on fluid type and installation. Turbine meters can cost from $\$ 300$ to $\$ 2,300$, depending on size.

Velocity - Vortex Shedding Meter. A vortex shedding meter senses flow disturbances around a stationary body (called a bluff body) positioned in the middle of the fluid stream. As fluid flows around the bluff body, eddies or vorticies are created downstream; the frequencies of these vortices are directly proportional to the fluid velocity. Figure 5.8 presents the typical vortex shedding meter.

As the vorticies are developed along the bluff body, they grow and are detached - it is this detachment that is electronically sensed and totalized. Because the vortex shedding meter has no moving parts, it is a very reliable method of natural gas measurement. As with the other non-positive displacement meters, the vortex shedding meter is used in very specific natural gas applications. Vortex shedding meters can be used on connection sizes of 1 to 12 inches and have accuracies in the 1 to 2 percent range. Vortex shedding meters are relatively expensive, typically costing between $\$ 500$ and $\$ 3,000$.

\subsubsection{Recording Intervals}

Due to the way natural gas is purchased and used, the data-recording interval is not as critical as it is for electricity. However, an ability to record gas use at least hourly, if not every 15 minutes, can afford some diagnostic capabilities. One such capability would be to diagnose the short-cycling of a boiler or other gas-using device; if the recording interval were too long, this frequent (and inefficient) on/off boiler operation would be masked in the longer recording interval. The best capability is to be able to modify the interval (at times to a shorter more refined interval) for temporary diagnostics and then return the setting to the longer more manageable interval. 


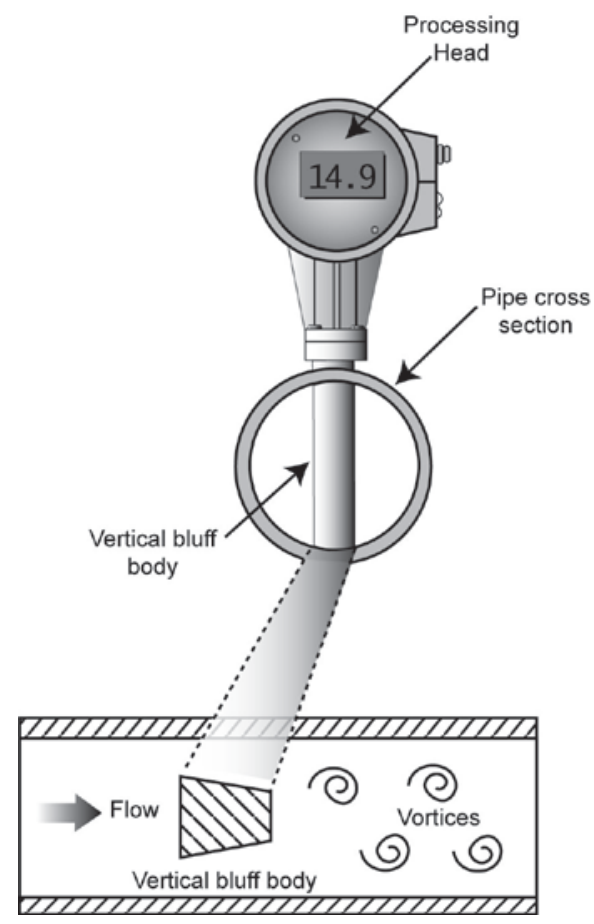

Figure 5.8. Typical Vortex Shedding Meter

\subsubsection{Natural Gas Meter Maintenance}

Depending on the meter technology, installation, and quality of gas delivered, natural gas meters generally require limited maintenance. Procedures followed should be those recommended by the manufacturer. Pending their availability, below are some general maintenance guidelines, presented by meter technology.

\section{Positive Displacement Meter}

Monthly Inspections

- All connections for gas leakage

- Noisy operation internal to meter

- Consistent and smooth register operation

- General meter cleanliness

Annual Inspections

- Calibration according to manufacturer's recommendation or if trended data indicate miscalibration.
Depending on the meter technology, installation, and quality of gas delivered, natural gas meters generally require limited maintenance. 


\section{Differential Pressure Meter}

Monthly Inspections

- All connections for gas leakage

- Abnormally loud or discontinuous sounds internal to meter

- Check for properly connected and sealed pressure taps

- General meter cleanliness

Annual Inspections

- Check orifice diameter and edges for wear, roughness, or material buildup

- Check venturi for cleanliness and corrosion at throat. Clean and smooth all internal surfaces.

- Check for well-connected and sealed pressure taps

- Calibration of differential pressure sensors/transmitters according to manufacturer's recommendation or if trended data indicate miscalibration.

\section{Velocity Meter}

Monthly Inspections

- All connections for gas leakage

- Abnormally loud or discontinuous sounds internal to meter

- General meter cleanliness

Annual Inspections

- Impeller blades should be checked for wear or damage

- Impeller bearings should be checked for wear

- Calibration of velocity meter according to manufacturer's recommendation or if trended data indicate miscalibration.

\subsubsection{Natural Gas Metering Data Output/Communications Considerations}

When specifying the natural gas flow

meter, the pulse calibration is a critical parameter.
At the whole-building level, where positive displacement diaphragm and rotary meters are the most common, the typical data output are calibrated pulses. While other output options are available (4 to 20 milliamp, 0-5 volt, Modbus, etc.), calibrated pulses are the most common and relatively easy to work with. Chapter 6 addresses in more detail the different output and communications options.

When specifying the natural gas flow meter, the pulse calibration is a critical parameter. Important in this specification is understanding the range of expected flow and necessary resolution of output. There are situations where 
too high of a pulse count (i.e., too high of a frequency) can result in saturation of the data logger or other collection device. A saturation condition usually results in loss of data and erroneous pulse counts. Therefore, meter and data logger vendors should be consulted when determining the appropriate pulse count and calibration.

\subsubsection{Natural Gas Meter Specification Considerations}

- Determine expected range of gas flows.

- Determine the accuracy requirements over the flow range - this will help define the necessary turndown ratio.

- Identify any physical installation requirements for meter location, straight lengths of piping, available communications, etc.

- Communication interoperatability - consider standardization on communication between meters and other data acquisition systems.

Do not overlook the effort it will take to create a process to collect, store, and archive the metering data.

- Specification considerations - at the outset, consider a formal specification development of future additions to ensure future compatibility.

- Data processing - how will the collected data be processed? Does the metering equipment vendor offer this function/service? Do not overlook the effort it will take to create a process to collect, store, and archive the data.

- Facility staff buy-in - make certain those staff that will be installing, maintaining, and most importantly, using the data have a voice in meter selection.

\subsubsection{Natural Gas Meter Selection Criteria}

Table 5.1 presents some of the more common natural gas metering technologies and key criteria for selection decisions (FEMP 2010; Carbon Trust 2005).

Table 5.1. Common Natural Gas Metering Technologies and Key Criteria

\begin{tabular}{|l|c|c|c|c|c|c|}
\hline \multicolumn{1}{|c|}{ Criteria } & $\begin{array}{c}\text { Positive } \\
\text { Displacement }\end{array}$ & Orifice & Venturi & Annubar & Turbine & $\begin{array}{c}\text { Vortex } \\
\text { Shedding }\end{array}$ \\
\hline Accuracy & Good & Moderate & Good & Good & Good & Good \\
\hline Turndown Ratio & $10: 1$ & $<5: 1$ & $<5: 1$ & $10: 1$ & $10: 1$ & $20: 1$ \\
\hline $\begin{array}{l}\text { Repeatability } \\
\text { Installation Ease }\end{array}$ & Good & Good & Good & Very Good & Low & Very good \\
\hline Pressure loss & Medium & Moderate & Low & Low & Moderate & Low \\
\hline Recalibration Needs & Infrequent & Frequent & Infrequent & Infrequent & Frequent & Infrequent \\
\hline Capital Cost & Low & Low & Moderate & Low & Moderate & Moderate \\
\hline Installed Cost & Moderate & Low & Moderate & Low & Moderate & Moderate \\
\hline Maintenance Cost & Low & High & Moderate & Low & Moderate & Low \\
\hline
\end{tabular}




\subsubsection{References}

Carbon Trust. 2005. Good Practices Guide: Reducing Energy Consumption Costs by Steam Metering. Available at: http://thecesh.com/wp-content/uploads/2010/05/GPG018.pdf.

Federal Energy Management Program (FEMP). 2007. FEMP Metering Training Course Session 2: Metering Technologies, Communications, and Data Storage. April 4, 2005. Available at: http://eere.pnnl.gov/femp/metering_webcast.stm.

Federal Energy Management Program (FEMP). 2010. Operations \& Maintenance Best Practices: A Guide to Achieving Operational Efficiency, Release 3.0, prepared by Pacific Northwest National Laboratory for the Federal Energy Management Program, U.S. Department of Energy, Washington, D.C. Available at: http://www1.eere.energy.gov/femp/pdfs/omguide_complete.pdf

New Buildings Institute (NBI). 2009. Advanced Metering and Information Systems. Report prepared for the U.S. Environmental Protection Agency. 


\subsection{Fluid Metering - Steam}

\subsubsection{Introduction}

When compared to other liquid flow metering, the metering of steam flow presents one of the most challenging metering scenarios. Most steam meters measure a volumetric flow of the steam and, unless this is done carefully, the physical properties of steam will impair the ability to measure and define a volumetric flow rate accurately.

Steam is defined as a compressible fluid; therefore, a reduction in pressure results in a reduction in density and vice versa. There is a similar relationship with temperature. While the temperature and pressure in steam lines can be relatively constant, in most applications, this is not the case. Changes in the system's dynamics, control system operation and instrument calibration can result in considerable differences between actual pressure/temperature and a meter's design parameters. Therefore, accurate steam flow measurement generally requires the measurement of the fluid's temperature and pressure, in addition to flow. This information is transmitted to an electronic device (either internal or external to the flow meter electronics) and the flow rate is corrected (or compensated) based on actual fluid conditions.

Besides the meter accuracy issues relating to pressure and temperature, The temperatures associated with steam flow measurement are often quite high and can affect the accuracy and longevity of metering additional steam properties add to the difficulties experienced in steam flow electronics. measurement. The temperatures associated with steam flow measurement are often quite high. These temperatures can affect the accuracy and longevity of metering electronics. Some metering technologies use close-tolerance moving parts that can be affected by moisture or impurities in the steam. Improperly designed or installed components can result in steam system leakage and impact plant safety. The erosive nature of poor-quality steam can damage steam flow sensing elements and lead to inaccuracies and/or device failure. These differences between steam flow metering and that of other fluid flow metering increase the importance of proper metering design, selection, and installation.

Finally, the challenges of metering steam can be somewhat simplified by instead measuring the condensed steam - the so-called condensate. The metering of condensate (i.e., high-temperature hot water) is a well-accepted practice, often less expensive and more reliable than steam metering. However, depending on the application, inherent inaccuracies in condensate metering stem from unaccounted for system steam losses. These losses are often difficult to find and quantify and thus affect condensate measurement accuracy.

\subsubsection{Steam Metering Technologies}

In general, measurement of the volumetric flow rate of a closed fluid system is a calculated or inferred value based on the simple relationship of velocity and area. A fluid's volume flow rate is equal to the product of the fluid's velocity and the pipe's cross-section. Since the pipe's cross-sectional area is a known constant, the fluid flow rate can be defined if its velocity can be quantified. This velocity value is then converted into a volumetric and/or mass flow rate. In general, volumetric metering approaches used in steam metering can be broken down into two operating designs: (1) differential pressure and (2) velocity metering technologies (FEMP 2007). 


\subsubsection{Differential Pressure Meters}

All differential pressure meters rely on the velocity-pressure relationship of flowing fluids for operation. Specifically, when an obstruction or orifice is placed in the path of a fluid, the fluid velocity will increase while its pressure decreases. It is this change in pressure that is measured and, via the relationship between pressure drop and the square of the flow, used to calculate the fluid flow rate. Two of the more common differential pressure devices used to measure steam are presented below.

Two of the more common differential pressure devices are the orifice meter and the annubar meter.

Differential Pressure - Orifice Meter. Historically, the orifice meter is one of the most commonly used meters to measure steam flow. The orifice meter for steam functions identically to that for natural gas flow. The meter has an orifice element that is typically a thin, circular metal disk held between two flanges in the fluid stream. The center of the disk is drilled with a specific-size hole, depending on the expected fluid flow parameters (e.g., pressure and flow range). As the fluid flows through the orifice, the restriction creates a pressure differential upstream and downstream of the orifice proportional to the fluid flow rate. This differential pressure is measured and a flow rate mathematically calculated based on the differential pressure and fluid temperature. Figure 5.9 is a diagram of a typical orifice meter.

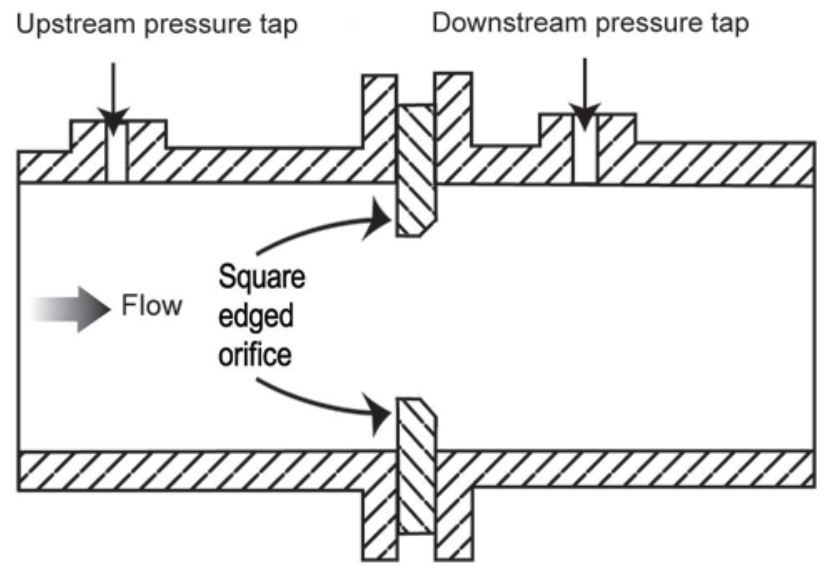

Figure 5.9. Typical Orifice Meter

\section{Orifice Plate Advantages/Challenges}

\section{Advantages:}

- Simplicity and durability

- Good accuracy over moderate turndown ratio

- Relatively inexpensive

- Limited recalibration needs

\section{Challenges:}

- Orifice needs to be regularly inspected for shape and size

- When mounted horizontally, dirt and scale can cause blockage/reduced flow through orifice

- Limited turndown
Orifice meters, by design, develop significant pressure drop within the system. The benefits of this technology (i.e., its relative compactness, its accuracy, and its simple function) should be traded off against the potential decrease pressure drop at the end use.

For steam metering, orifice meters are commonly used to monitor boiler steam production, amounts of steam delivered to a process or tenant, or in mass balance activities for efficiency calculation or trending. 
For proper function, the orifice meter requires smooth flow through the orifice. For this to be assured, it requires a significant section of straight pipe both upstream and downstream of the meter. While these lengths may vary by manufacturer, typical lengths fall between 15 to 25 diameters upstream and 5 to 15 diameters downstream. As with most differential pressure meters, the turndown ratio of the orifice plate is usually limited to 5:1; thus, for steam systems with highly variant flow rates, this technology may not be the most accurate choice.

Orifice meters are commonly used on line sizes from 0.25 to 4 inches or larger. These meters have accuracies ranging from 0.25 to 2 percent, depending on the fluid, type of orifice, and installation. Orifice meters range in cost from $\$ 300$ to $\$ 5,000$, with the higher cost systems associated with larger and more accurate meters.

Differential Pressure - Annubar Meter. The annubar flow meter (a variation of the simple pitot tube) also takes advantage of the velocitypressure relationship of flowing fluids. In this case, the device causing the change in pressure is a pipe inserted into the steam flow. Contained within this pipe are two smaller tubes with holes or ports evenly spaced along the length. Figure 5.10 presents the components of the annubar flow meter. When properly installed, one tube faces directly upstream and one downstream. These tubes and ports become the pressure detection points for the meter; the upstream-facing ports measure the

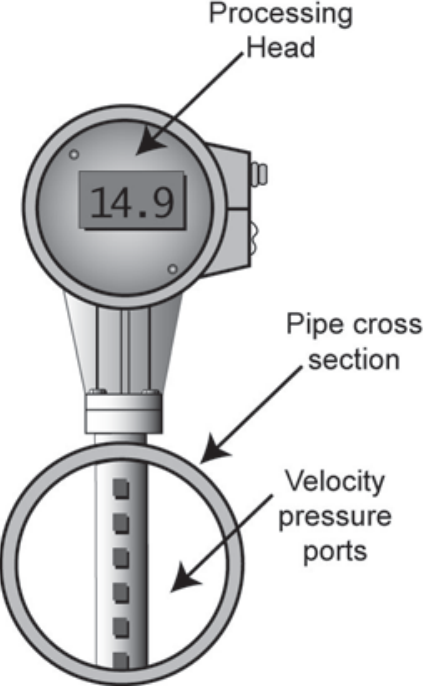

Figure 5.10. Typical Annubar Meter flowing pressure (i.e., velocity pressure) and the downstream port measures the static pressure. Using these measured pressure values and the previously mentioned pressure-flow relationship, the flow rate is calculated.

Annubar flow meters have a turndown ratio of up to 10:1 and are relatively easy to install. These meters can make measurements on pipe sizes from 2 to 100 inches to an accuracy of 2 percent.

Accuracy will vary by installation and requires strict adherence to manufacturer's requirements on straight-pipe-lengths upstream and downstream of the meter. Annubar meters can cost between

Annubar Flow Meter Advantages/Challenges

Advantages:

- Limited resistance to flow

- Good accuracy and repeatability

- Lower installation cost - particularly on larger pipe diameters

- Expanded turndown ratio.

Challenges:

- Installation needs proper lengths of pipe for accurate measurements.

$\$ 1,000$ to $\$ 3,000$, depending on size and accuracy.

\subsubsection{Velocity Meters}

The second general category of steam flow measurement employs the velocity of the steam as the measurement metric. Velocity meters take advantage of the linear relationship between fluid velocity and flow. The two main type of velocity meters for steam flow, turbine and vortex shedding, both sense some flow characteristic directly proportional to the fluid's velocity. In the case of the turbine meter, it is the velocity of the turbine, in the case of the vortex meter, it is the generation frequency of vorticies. Both meter types are described below relative to their steam applications. 


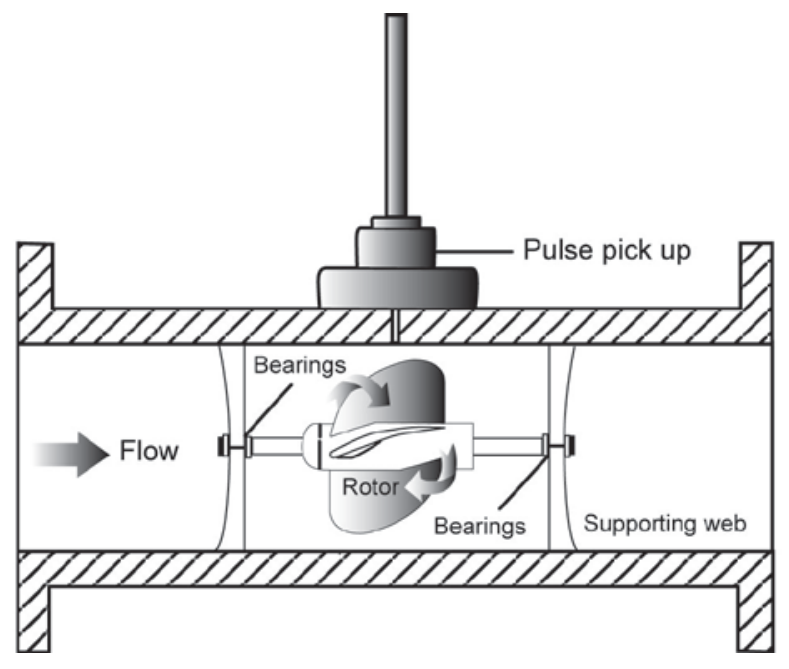

Figure 5.11. Typical Turbine Meter

Velocity - Turbine Meter. The turbine meter for steam flow employs a multi-blade impellorlike device is located in, and horizontal to, the fluid stream. As the fluid passes through the turbine blades, the impellor rotates at a speed related to the fluid's velocity. Blade speed can be sensed by a number of techniques including magnetic pick-up, mechanical gears, and photocell. The pulses generated as a result of blade rotation are directly proportional to fluid velocity, and, hence, flow rate. Figure 5.11 details the components of a typical turbine meter. It should be noted that there are a variety of turbine designs and mounting configurations, of which Figure 5.11 highlights the concept.

Because the turbine meter has mechanical elements in the fluid stream, these meters can be susceptible to wear and resulting inaccuracies. Of particular concern are the bearings that, if exposed to corrosive or contaminated steam flows, can quickly wear and result in inaccuracy. The steam applications for turbine meters are typically larger industrial metering functions. As with the orifice meter, the proper function the turbine meter requires smooth flow. For this to be assured, the meter requires a significant section of straight pipe for upstream and downstream of the meter. While these lengths may vary by manufacturer, typical lengths fall between 15 to 25 diameters upstream and 5 to 15 downstream.

\section{Vortex Meter Advantages/Challenges}

\section{Advantages:}

- Good accuracy over large turndown ratio

- Higher reliability owing to the lack of any moving parts

- With high-quality steam, recalibration need is negated

\section{Challenges:}

- Meter needs to be isolated from mechanical vibration

- Longer lengths of straight pipe for accurate operation

Turbine meters are used on line sizes ranging from 2 to 20 inches and have accuracies in the range of 0.5 to 1.0 percent depending on fluid type and installation and turndown ratio of up to 10:1. Turbine meters can cost from $\$ 300$ to $\$ 2,300$, depending on size.

Velocity - Vortex Shedding Meter. The steam vortex shedding meter senses flow disturbances around a stationary body (called a bluff body) positioned in the middle of the fluid stream. As the fluid, steam in this case, flows around the bluff body, eddies or vorticies are created downstream. A sensing element, usually a piezo-electric element, detects the frequencies of these vorticies, which are directly proportional to the fluid velocity. Figure 5.12 presents the typical vortex shedding meter. As the vorticies are developed along the bluff body, they grow and are detached - it is this detachment that is sensed and totalized. 
Because the vortex shedding meter has no moving parts, it is a very reliable method of steam-flow measurement. In addition, because the meter measures flow directly (versus the exponential relationship of differential meters) a higher turndown ratio can be achieved - on the order of 20:1.

Vortex shedding meters can be used on line sizes of 1 to 12 inches and has accuracies in the 1 to 2 percent range. Vortex shedding meters are relatively expensive, typically costing between $\$ 500$ and $\$ 3,000$.

\subsubsection{Recording Intervals}

Due to the way steam is generated and/or purchased and used, the data-recording interval is not as critical as it is for electricity. However, an ability to record steam use at least hourly, if not every 15 minutes (or less), can afford some diagnostic capabilities. One such capability would be to diagnose excessive line loss or steam leakage. In this case, a detailed recording of high off-hours (low-load) steam production and frequency could be very

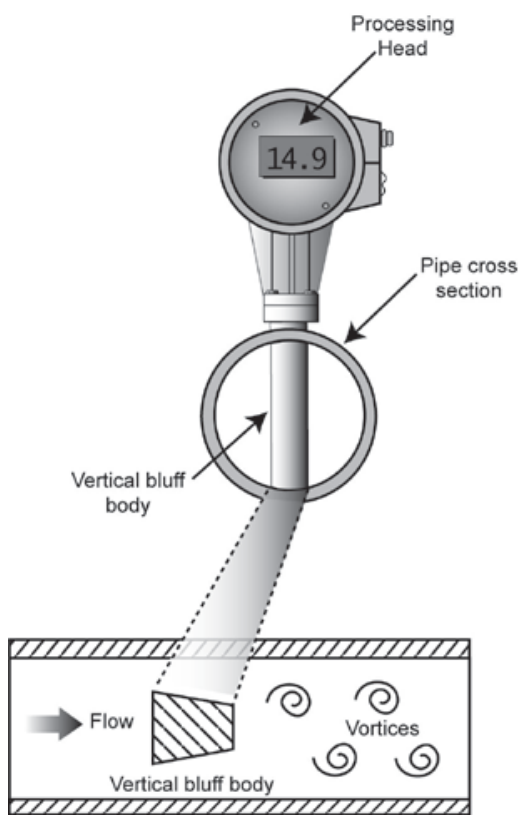

Figure 5.12. Typical Vortex Shedding Meter diagnostic. If the recording interval were too long, these losses may be masked in the longer recording interval. The best capability is to be able to modify the interval (at times to a shorter more refined interval) for temporary diagnostics and then return the setting to the longer more manageable interval.

\subsubsection{Steam Meter Maintenance}

Depending on the meter technology, installation, and quality of steam generated, steam meters can require a significant amount of maintenance attention. Procedures followed should be those as recommended by the manufacturer. Pending their availability, below are some general maintenance guidelines presented by meter technology.

\section{Differential Pressure Meters}

Monthly Inspections

- All connections for steam leakage

- Abnormally loud or discontinuous sounds internal to meter

- Check for properly connected and sealed pressure taps

- General meter cleanliness

Depending on the meter technology, installation, and quality of steam generated, steam meters can require a significant amount of maintenance

Annual Inspections attention.

- Check orifice diameter and edges for wear, roughness, or material buildup

- Check pressure parts for wear, roughness, or material buildup 
- Check for properly connected and sealed pressure taps

- Calibration of differential pressure sensors/transmitters according to manufacturer's recommendation or if trended data indicate miscalibration.

\section{Velocity Meters}

Monthly Inspections

- All connections for steam leakage

- Abnormally loud or discontinuous sounds internal to meter

- General meter cleanliness

Annual Inspections

- Impeller blades should be checked for wear or damage

- Impeller bearings should be checked for wear

- Calibration of velocity meter according to manufacturer's recommendation or if trended data indicate miscalibration.

\subsubsection{Steam Metering Data Output/Communications Options}

The most common outputs of steam metering devices are scalable analog signals of either 4 to $20 \mathrm{~mA}$ or 0 to 5 volts dc. These outputs typically are collected and processed using a flow computer/analysis device integral to the meter. This device takes the measurement signals (pressure, differential pressure, and temperature) and convert these to a compensated flow

The most common rate.

outputs of steam

metering devices

are scalable analog

signals.

When specifying a steam meter, the flow computer/analysis device is typically an option with some array of alternatives for analysis and presentation. The output of the flow computer/analysis device is typically a scalable signal or pulse that can be transferred to a data acquisition/logger system for collection and further analysis or trending. Chapter 6 addresses in more detail the different output and communications options.

\subsubsection{Steam Meter Specification Considerations}

- Determine generated steam characteristics (i.e., dry, wet, saturated, and the corresponding temperatures) - this will make sure compatible meters are considered.

- Determine expected range of steam flows.

- Determine the accuracy requirements over the flow range - this will help define the necessary turndown ratio.

- Communication interoperatability - consider standardization on communication between meters and other data acquisition systems. 
- Specification considerations - at the outset, consider a formal specification development so future additions to ensure future compatibility.

- Data processing - how will the collected data be processed? Does the metering equipment vendor offer this function/service? Do not overlook the effort it will take to create a process to collect, store, and archive the data.

- Facility staff buy-in - make certain those staff that will be installing, maintaining, and most importantly, using the data have a voice in meter selection.

\subsubsection{Steam Meter Selection Criteria}

Table 5.2 presents some of the more common steam metering technologies and key criteria for selection decisions (FEMP 2010; Carbon Trust 2005).

Table 5.2. Common Steam Metering Technologies and Key Criteria

\begin{tabular}{|l|c|c|c|c|}
\hline \multicolumn{1}{|c|}{ Criteria } & Orifice & Annubar & Turbine & $\begin{array}{c}\text { Vortex } \\
\text { Shedding }\end{array}$ \\
\hline Accuracy & Moderate & Good & Good & Good \\
\hline Turndown Ration & $<5: 1$ & $5: 1$ & $10: 1$ & $20: 1$ \\
\hline Repeatability & Good & Good & Low & Very good \\
\hline Installation Ease & Easy & Easy & Challenging & Moderate \\
\hline Pressure loss & Moderate & Low & Moderate & Low \\
\hline Recalibration Needs & Frequent & Infrequent & Frequent & Infrequent \\
\hline Capital Cost & Low & Low & Moderate & Moderate \\
\hline Installed Cost & Low & Low & Moderate & Moderate \\
\hline Maintenance Cost & High & Low & Moderate & Low \\
\hline
\end{tabular}

\subsubsection{References}

Carbon Trust. 2005. Good Practices Guide: Reducing Energy Consumption Costs by Steam Metering. Available at: http://thecesh.com/wp-content/uploads/2010/05/GPG018.pdf.

Federal Energy Management Program (FEMP). 2007. FEMP Metering Training Course Session 2: Metering Technologies, Communications, and Data Storage. April 4, 2005. Available at: http://eere.pnnl.gov/femp/metering_webcast.stm.

Federal Energy Management Program (FEMP). 2010. Operations \& Maintenance Best Practices: A Guide to Achieving Operational Efficiency, Release 3.0, prepared by Pacific Northwest National Laboratory for the Federal Energy Management Program, U.S. Department of Energy, Washington, D.C. Available at: http://www1.eere.energy.gov/femp/pdfs/omguide_complete.pdf. 
This page intentionally left blank. 


\subsection{Fluid Metering - Water}

\subsubsection{Introduction}

The physical properties associated with water lend themselves to a wide variety of metering technologies. The lack of high-temperature and/or two-phase flow allows use of metering technologies not suitable for other applications (e.g., steam or high-temperature hot water). Because the metering of water is generally concerned with the quantification of flow volume, and not energy content, lower-cost metering options can be used. The specific metering option chosen will depend on a number of factors including, but not limited to, current design, budget, accuracy requirements, minimum flow, range of flow, and maximum flow.

\subsubsection{Water Metering Technologies}

In general, measurement of the volumetric flow rate of a closed fluid system is a calculated or inferred value based on the simple relationship of velocity and area. A fluid's volume flow rate is equal to the product of the fluids velocity and the pipe's cross-section. Since the pipe's cross-sectional area is a known constant, the fluid flow rate can be defined if its velocity can be quantified. Some meter styles measure fluid flow directly by incrementally moving the fluid through a known volume (positive displacement meters). These volumetric increments are counted by a variety of mechanical/electronic approaches and the flow rate converted to the desired unit (e.g., gpm). In general, volumetric water metering designs can be broken down into three general operating designs: (1) positive displacement, (2) differential pressure, and (3) velocity (FEMP 2007). Some meters have been specifically designed for applications generally falling outside those required for general building utility metering needs, for instance, mass meters like thermal or electromagnetic meters. The balance of this section will focus on the three operating designs mentioned above.

\subsubsection{Positive Displacement Meters}

As the name implies, a positive displacement meter functions by the action of the fluid physically displacing the measuring mechanism. Several different designs exist that determine water flow rate based on how fast a known volume of water enters and exits the meter. Nutating-disk, oval-gear, piston, and rotary-vane are all styles of positive displacement meters. Of relevancy to potable water measurement, the predominant positive displacement technology is the nutating-disk flow meter.

Positive Displacement - Nutating-Disk Meter. Because of its ease of installation and relatively low cost, nutating-disk meters are the most common potable water metering devices for up to 3-inch connections.

The nutating disk meter consists of a disk mounted on a spherically shaped head and housed in a measuring chamber. As the fluid flows through the meter passing on either side of the disk, it imparts a rocking or nutating motion to the disk. This motion is then transferred to a shaft mounted perpendicular to the disk. It is this shaft that traces out a circular motion - transferring this action to a register that records flow. 
For potable water metering, nutating disk meters are typically found at the service entrances to buildings and homes. These meters are most often used on pipe sizes up to 3 inches and have an accuracy in the range of 0.5 to 1.0 percent. Nutating disk meters cost from $\$ 80$ to $\$ 300$, depending on the size.

\subsubsection{Differential Pressure Meters}

All differential pressure meters rely on the velocity-pressure relationship of flowing fluids for operation. Specifically, when an obstruction or orifice is placed in the path of a fluid, the fluid velocity will increase while its pressure decreases. It is this change in pressure that is measured and, via the relationship between pressure drop and the square of the flow, used to

All differential pressure meters rely on the velocitypressure relationship of flowing fluids for operation. calculate the fluid flow rate. There are a variety of differential pressure devices useful for water metering; three of the more common devices are described below.

Differential Pressure - Orifice Meter. Although various style orifices are manufactured, the basic design and operation remain the same. The orifice element is typically a thin, circular metal disk held between two flanges in the fluid stream. The center of the disk is drilled with a specific-size hole, depending on the expected fluid flow parameters (e.g., pressure and flow range). As the fluid flows through the orifice, the restriction creates a pressure differential upstream and downstream of the orifice proportional to the fluid flow rate. This differential pressure is measured and a flow rate mathematically calculated based on the differential pressure and fluid temperature. Figure 5.13 presents a diagram of a typical orifice meter.

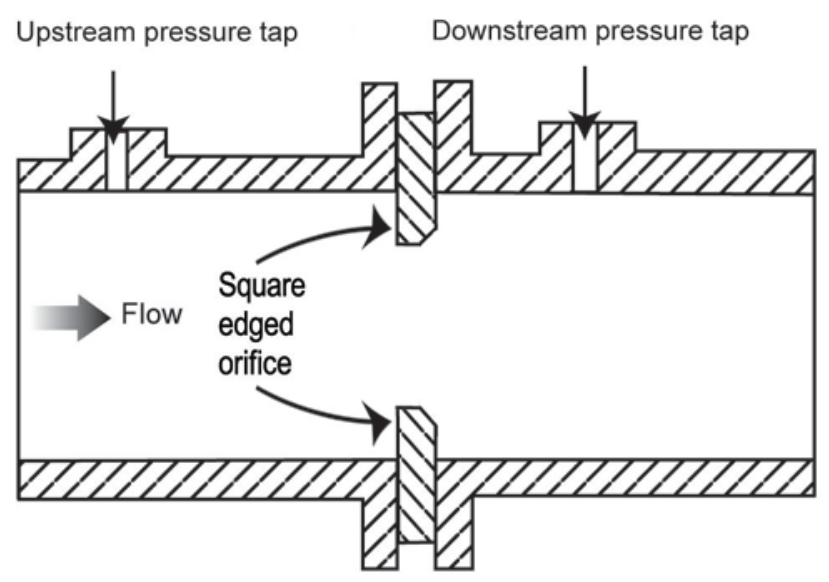

Figure 5.13. Typical Orifice Meter

Orifice meters, by design, develop significant pressure drop within the system. The benefits of this technology (i.e., its relative compactness, its accuracy, and its simple function) should be traded off against the potential decrease pressure drop at the end use.

Orifice meters are commonly used on line sizes from 0.25 to 4 inches. These meters have accuracies ranging from 0.25 to 2 percent, depending on the fluid, type of orifice, and installation. Orifice meters range in cost from $\$ 300$ to $\$ 5,000$, with the higher cost systems associated with larger and more accurate meters. 
Differential Pressure - Venturi Meter. Similar in function to the orifice meter, the venturi meter takes advantage of the same velocity-pressure relationship (change in pressure is proportional to the square of the velocity). In this case, the device causing the change in pressure is not a sharp-edged orifice but rather a section of pipe that gently converges to a small-diameter area (called a throat) before diverging back to the full pipe diameter. Figure 5.14 presents the operation of a typical venturi meter.

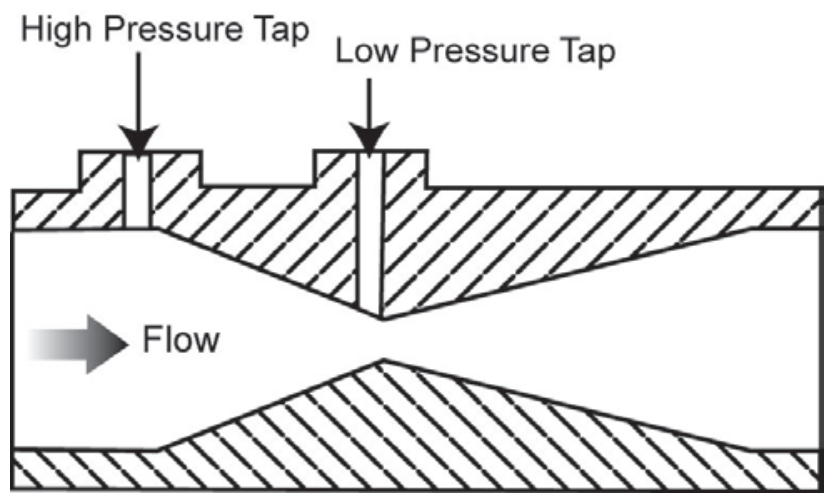

Figure 5.14. Typical Venturi Meter

The benefit of the venturi meter over the orifice meter lies in the reduced pressure loss experienced by the fluid. In situations where the cost to pump a fluid is high, this benefit can represent a significant savings over the life of the system. An additional benefit of the venture meter is its durability - it does not have the "sharp edge" profile of the orifice meter; therefore, it does not suffer from potential erosion issues and has a greater ability to accurately meter contaminated on non-homogeneous fluids. As with orifice plate meters, venturi meters are used mostly in specialty potable water applications where size, space, and/or accuracy dictate their use.

Venturi meters can be used on connection sizes ranging from 0.25 to 4 inches and have accuracies in the range of 0.25 to 2.0 percent, depending on fluid type and installation. Venturi meters can cost from $\$ 1,000$ to $\$ 5,000$.

\subsubsection{Velocity Meters}

Another means to determine fluid flow is by a technique that directly relates to the fluid's velocity. Velocity meters determine fluid flow by measuring a representation of the flow directly. Because the fluid's velocity is measured (i.e., not the square-root relationship to determine velocity as with differential pressure meters), velocity meters can have better accuracy and usually have better turndown ratios than other meter types.

Velocity - Turbine Meter. Turbine meters operate as their name implies. A multi-blade impellor-like device is located in, and horizontal to, the fluid stream. As the fluid passes through the turbine blades, the impellor rotates at a speed related to the fluid's velocity. Blade speed can be sensed by a number Because the turbine meter has mechanical elements in the fluid stream, these meters can be susceptible to wear and resulting inaccuracies. of techniques including magnetic pick-up, mechanical gears, and photocell. The pulses generated as a result of blade rotation are directly proportional to 


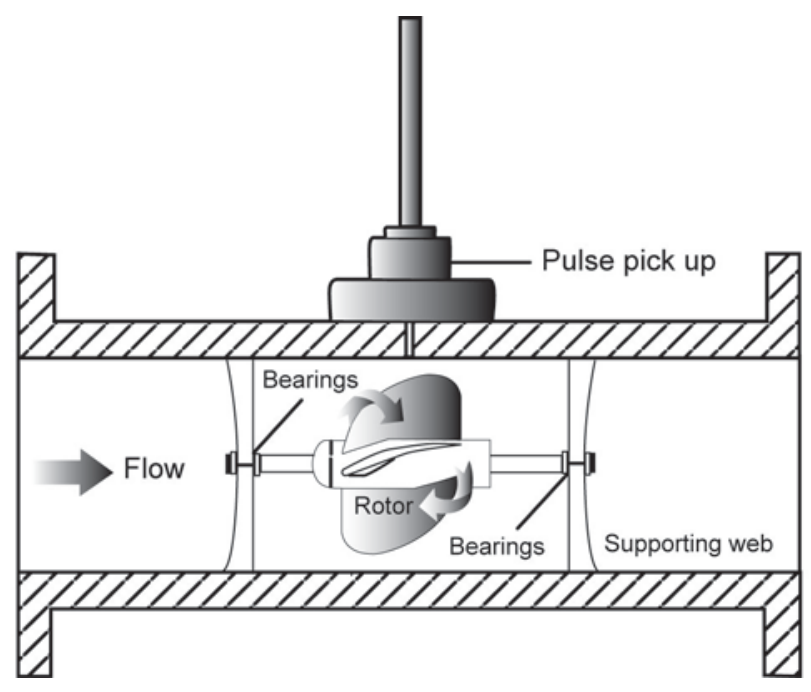

Figure 5.15. Typical Turbine Meter fluid velocity, and hence flow rate.

Figure 5.15 details the components of a typical turbine meter. It should be noted that there are a variety of turbine designs and mounting configurations, of which Figure 5.15 highlights the concept.

Because the turbine meter has mechanical elements in the fluid stream, these meters can be susceptible to wear and resulting inaccuracies. Of particular concern are the bearings that, if exposed to corrosive or contaminated fluids, can quickly wear and result in inaccuracy. The potable water applications for turbine meters are typically for larger industrial metering functions. Turbine meters are used on connection sizes

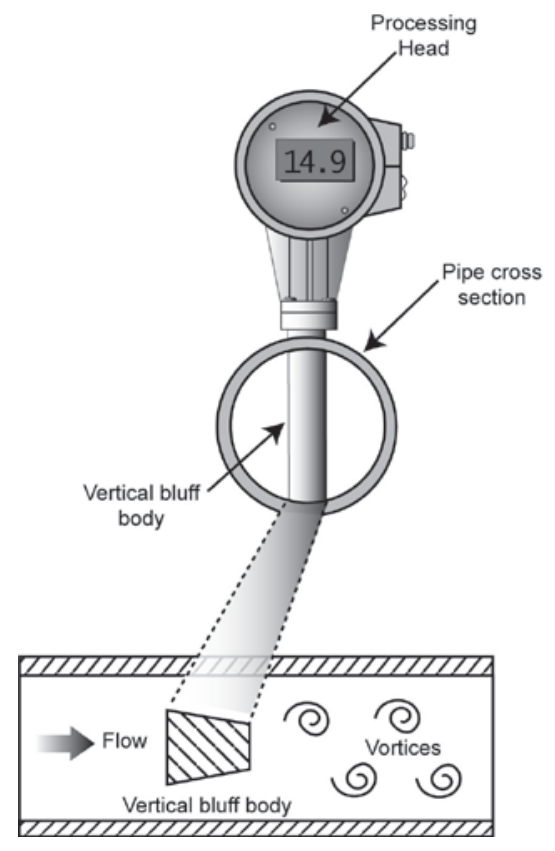

Figure 5.16. Typical Vortex Shedding Meter

Vortex shedding meters can be used on connection sizes of 1 to 12 inches and have accuracies in the 1 to 2 percent range. ranging from 2 to 20 inches and have accuracies in the range of 0.5 to 1.0 percent, depending on fluid type and installation. Turbine meters can cost from $\$ 300$ to $\$ 2,300$, depending on size.

Velocity - Vortex Shedding Meter. A vortex shedding meter senses flow disturbances around a stationary body (called a bluff body) positioned in the middle of the fluid stream. As fluid flows around the bluff body, eddies or vorticies are created downstream; the frequencies of these vorticies are directly proportional to the fluid velocity. Figure 5.16 presents the typical vortex shedding meter.

As the vorticies are developed along the bluff body, they grow and are detached - it is this detachment that is electronically sensed and totalized. Because the vortex shedding meter has no moving parts, it is a very reliable method of potable water measurement. As with the other non-positive displacement meters, the vortex shedding meter is used in very specific potable water applications. Vortex shedding meters can be used on connection sizes of 1 to 12 inches and have accuracies in the 1 to 2 percent range. Vortex shedding meters typically cost between $\$ 500$ and $\$ 3,000$.

Velocity - Ultrasonic Meters. Ultrasonic meters function through the basic relationship between the velocity of a fluid and its accompanying frequency shift - also known as the Doppler effect. One of the most attractive aspects of ultrasonic flow meters is they are completely non-intrusive. That is, this technology does not require any permanent modifications or penetrations to piping or disruption of service for installation. All ultrasonic meters mount on 
the outside of the piping and can be used as both temporary and permanent metering.

There are two styles of ultrasonic flow meters available: Doppler meters and transit time meters. The Doppler meters function by measuring the frequency shift of the moving fluid to calculate flow. This technology works best when the fluid has suspended solids, bubbles, or other particles to reflect the ultrasonic signal. Figure 5.17 presents the function of the Doppler ultrasonic meter.

The transit time meter makes use of a transmitter and receiver mounted on opposite sides of the pipe. As fluid moves through the system, the first transducer sends a signal and the second receives it. The time it takes for the signal to arrive is proportionate to the flow rate. Different from the Doppler meter, the transit time meter needs clean and viscous liquids for best accuracy. Figure 5.18 presents the function of the transit time ultrasonic meter.

Because ultrasonic meters have no moving parts, they are very reliable. These meters can be used on connection sizes up to 20 inches or larger and have accuracies ranging from 1 to 5 percent. Both types of ultrasonic meters are relatively expensive, costing between $\$ 1,500$ and $\$ 6,500$.

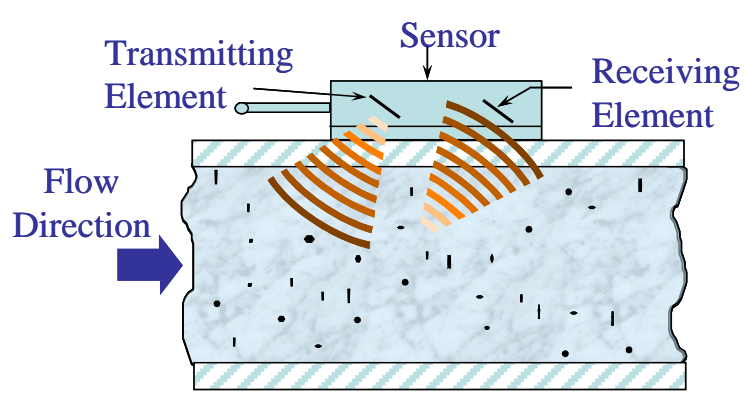

Figure 5.17. Doppler Ultrasonic Flow Meter

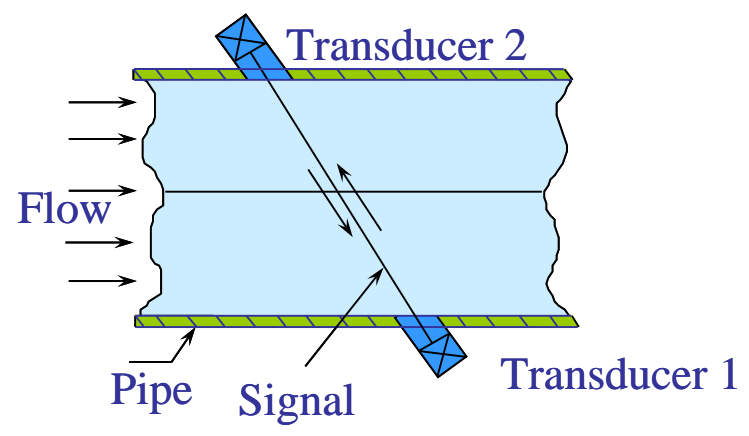

Figure 5.18. Transit Time Ultrasonic Flow Meter

\subsubsection{Recording Intervals}

Due to the way water is usually purchased and used, the data-recording interval is not as critical as it is for electricity. However, an ability to record water use at least hourly, if not every 15 minutes, can afford some diagnostic capabilities. One such capability would be to diagnose proper water flows and volumes based on known water uses. Another excellent use is leak detection where higher resolution (and short intervals) can be very diagnostic. The best capability is to be able to modify the interval (at times to a shorter more refined interval) for temporary diagnostics and then return the setting to the longer more manageable interval.

\subsubsection{Water Meter Maintenance}

Depending on the meter technology, installation, and quality of water delivered, water meters generally require limited maintenance. Procedures followed should be those as recommended by the manufacturer. Pending their availability, below are some general maintenance guidelines presented by meter technology. 


\section{Positive Displacement Meter}

Monthly Inspections

- All connections for water leakage

- Noisy operation internal to meter

- Consistent and smooth register operation

- General meter cleanliness

Annual Inspections

- Calibration according to manufacturer's recommendation or if trended data indicate miscalibration.

\section{Differential Pressure Meter}

Monthly Inspections

- All connections for water leakage

- Abnormally loud or discontinuous sounds internal to meter

- Check for properly connected and sealed pressure taps

- General meter cleanliness

Annual Inspections

- Check orifice diameter and edges for wear, roughness, or material buildup

- Check venturi for cleanliness and corrosion at throat. Clean and smooth all internal surfaces.

- Check for well-connected and sealed pressure taps

- Calibration of differential pressure sensors/transmitters according to manufacturer's recommendation or if trended data indicate miscalibration.

\section{Velocity Meter}

Depending on the meter technology, installation, and quality of water delivered, water meters generally require limited maintenance.
Monthly Inspections

- All connections for water leakage

- Abnormally loud or discontinuous sounds internal to meter

- General meter cleanliness

Annual Inspections

- Impeller blades should be checked for wear or damage

- Impeller bearings should be checked for wear

- Calibration of velocity meter according to manufacturer's recommendation or if trended data indicate miscalibration. 


\section{Ultrasonic Meter}

Monthly Inspections

- Transducer positions and attachment to piping

- Cleanliness of transducer/piping interface

- Verification of piping isolation (i.e., no vibration in metering section)

- General meter cleanliness

Annual Inspections

- Calibration of meter according to manufacturer's recommendation or if trended data indicate miscalibration.

\subsubsection{Water Metering Data Output/Communications Considerations}

At the whole-building level, where nutating disk meters are the most common, the typical data output is calibrated pulses. While other output options are available (4 to 20 milliamp, 0 to 5 volt, Modbus, etc.), calibrated pulses are the most common and relatively easy to work with. Chapter 6 addresses in more detail the different output and communications options.

When specifying the potable water flow meter, the pulse calibration is a critical parameter. Important in this specification is an understanding of the range of expected flow and necessary resolution of output. There are situations where too high of a pulse count (i.e., too high of a frequency) can result in saturation of the data logger or other collection device. A saturation condition usually results in loss of data and erroneous pulse counts; therefore, it needs to be avoided. Meter and data logger vendors should be consulted when determining the appropriate pulse count and calibration.

\subsubsection{Water Meter Selection Considerations}

When specifying the water flow meter, the pulse calibration is a critical parameter.

There are situations where too high of a pulse count can result in saturation of the data logger or other collection device.

- Determine expected range of potable water flows and pipe sizes.

- Determine the accuracy requirements over the flow range.

- Identify any physical installation requirements for meter location, straight lengths of piping, available communications, etc.

- Communication interoperatability - consider standardization on communication between meters and other data acquisition systems.

- Specification considerations - at the outset, consider a formal specification development so future additions to ensure future compatibility.
Determining the accuracy requirements over the flow range is one of the considerations for selecting a meter for

water. 
- Data processing - how will the collected data be processed? Does the metering equipment vendor offer this function/service? Do not overlook the effort it will take to create a process to collect, store, and archive the data.

- Facility staff buy-in - make certain those staff that will be installing, maintaining, and most importantly, using the data, have a voice in meter selection.

\subsubsection{Water Meter Selection Criteria}

Table 5.3 presents some of the more common water metering technologies and key criteria for selection decisions (FEMP 2010; Carbon Trust 2005).

Table 5.3. Common Water Metering Technologies and Key Criteria

\begin{tabular}{|l|c|c|c|c|c|c|}
\hline \multicolumn{1}{|c|}{ Criteria } & $\begin{array}{c}\text { Positive } \\
\text { Displacement }\end{array}$ & Orifice & Venturi & Turbine & $\begin{array}{c}\text { Vortex } \\
\text { Shedding }\end{array}$ & $\begin{array}{c}\text { Ultrasonic } \\
\text { Dop/TT }\end{array}$ \\
\hline Accuracy & Good & Moderate & Good & Good & Good & Moderate \\
\hline Turndown Ratio & $10: 1$ & $<5: 1$ & $<5: 1$ & $10: 1$ & $20: 1$ & $10: 1 / 20: 1$ \\
\hline Repeatability & Good & Good & Good & Low & Very good & Good \\
\hline Installation Ease & Easy & Easy & Moderate & Challenging & Moderate & Very easy \\
\hline Pressure Loss & Medium & Moderate & Low & Moderate & Low & None \\
\hline Recalibration Needs & Infrequent & Frequent & Infrequent & Frequent & Infrequent & Moderate \\
\hline Capital Cost & Low & Low & Moderate & Moderate & Moderate & High \\
\hline Installed Cost & Moderate & Low & Moderate & Moderate & Moderate & Low \\
\hline Maintenance Cost & Low & High & Moderate & Moderate & Low & Low \\
\hline
\end{tabular}

\subsubsection{References}

Carbon Trust. 2005. Good Practices Guide: Reducing Energy Consumption Costs by Steam Metering. Available at: http://thecesh.com/wp-content/uploads/2010/05/GPG018.pdf.

Federal Energy Management Program (FEMP). 2007. FEMP Metering Training Course Session 2: Metering Technologies, Communications, and Data Storage. April 4, 2005. Available at: http://eere.pnnl.gov/femp/metering_webcast.stm.

Federal Energy Management Program (FEMP). 2010. Operations \& Maintenance Best Practices: A Guide to Achieving Operational Efficiency, Release 3.0, prepared by Pacific Northwest National Laboratory for the Federal Energy Management Program, U.S. Department of Energy, Washington, D.C. Available at: http://www1.eere.energy.gov/femp/pdfs/omguide_complete.pdf. 


\subsection{Fluid Metering - High-Temperature Hot Water/Chilled Water}

\subsubsection{Introduction}

Although the flow metering principles remain the same, whether metering hot or chilled water, fluid temperature will affect final meter selection. To ensure the accuracy of most volumetric metering, water temperature must be maintained below saturation temperature related to the fluid pressure. Provided this relationship is maintained, the hardware required to meter volumetric flow rates of hot and cold water changes very little. However, not all flow metering equipment is designed to be used in high-temperature and/or high-pressure environments. Hot water will require selecting metering equipment designed to be used in the application's environmental conditions. Although a common practice in steam metering, compensating flow rates due to fluctuations in pressure and/or temperature is seldom done; pressure/ temperature variations only slightly affect the metering accuracy of water if maintained below saturation conditions.

Common applications of high-temperature hot water metering include high-temperature hot water distribution/heating systems and metering of condensate-return systems. Common chilled water metering applications include central chilled water distribution or packaged chiller systems.

\subsubsection{High-Temperature Hot Water/Chilled Water Technologies}

High-temperature and chilled water metering technologies are consistent with those presented in the previous section (FEMP 2007). However, the one caveat in selecting a meter for a high-temperature environment is verifying the meter is approved to operate in the expected range. Many manufacturers offer products for both standard and high-temperature environments - making sure the meter you have selected is applicable for the operating temperature range is critical to its performance and accuracy.

\subsubsection{Positive Displacement Meters}

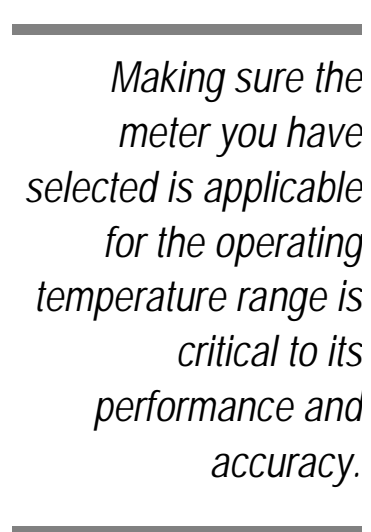
the fluid physically displacing the measuring mechanism. Several different designs exist that determine water flow rate based on how fast a known volume of water enters and exits the meter. Nutating-disk, oval-gear, piston, and rotary-vane are all styles of positive displacement meters. Of relevancy to high temperature and chilled water measurement, the predominant positive displacement technology is the nutating disk flow meter.

Positive Displacement - Nutating Disk Meter. Because of its ease of installation and relatively low cost, nutating disk meters are the most common of the positive displacement technologies for up to 3-inch connections.

The nutating disk meter consists of a disk mounted on a spherically-shaped head and housed in a measuring chamber. As the fluid flows through the meter passing on either side of the disk, it imparts a rocking or nutating motion to the disk. This motion is then transferred to a shaft mounted perpendicular to the disk. It is this shaft that traces out a circular motion - transferring this action to a register that records flow. 
These meters are most often used on pipe sizes up to 3 inches and have an accuracy in the range of 0.5 to 1.0 percent. Nutating disk meters cost from $\$ 80$ to $\$ 300$ - depending on the size. When considering a nutating disk meter for high-temperature fluids, make certain the meter is rated for the expected temperature range.

\subsubsection{Differential Pressure Meters}

All differential pressure meters rely on the velocity-pressure relationship of flowing fluids for operation. Specifically, when an obstruction or orifice is placed in the path of a fluid, the fluid velocity will increase while its pressure decreases. It is this change in pressure that is measured and, via the relationship between pressure drop and the square of the flow, used to

All differential

pressure meters rely

on the velocity-

pressure relationship

of flowing fluids for

operation. calculate the fluid flow rate. There are a variety of differential pressure devices useful for high-temperature/chilled water metering; two of the more common devices are described below.

Differential Pressure - Orifice Meter. Although various style orifices are manufactured, the basic design and operation remain the same. The orifice element is typically a thin, circular metal disk held between two flanges in the fluid stream. The center of the disk is drilled with a specific-size hole, depending on the expected fluid flow parameters (e.g., pressure and flow

range). As the fluid flows through the orifice, the restriction creates a pressure differential upstream and downstream of the orifice proportional to the fluid flow rate. This differential pressure is measured and a flow rate mathematically calculated based on the differential pressure and fluid temperature. Figure 5.19 presents a diagram of a typical orifice meter.

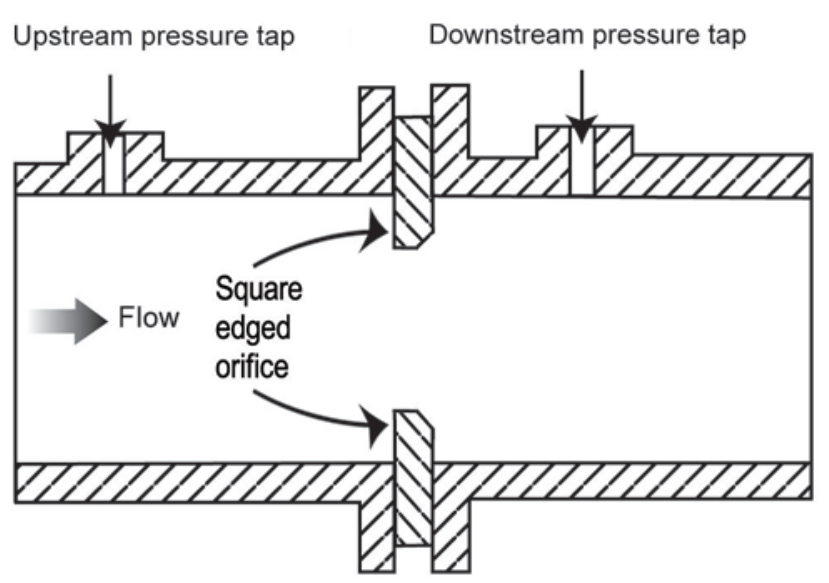

Figure 5.19. Typical Orifice Meter
Orifice meters, by design, develop significant pressure drop within the system. The benefits of this technology (i.e., its relative compactness, its accuracy, and its simple function) should be traded off against the potential decrease pressure drop at the end use.

Orifice meters are commonly used on line sizes from 0.25 to 4 inches. These meters have accuracies ranging from 0.25 to 2 percent depending on the fluid, type of orifice, and installation. Orifice meters range in cost from $\$ 300$ to $\$ 5,000$, with the higher cost systems associated with larger and more accurate meters.

Differential Pressure - Venturi Meter. Similar in function to the orifice meter, the venturi meter takes advantage of the same velocity-pressure relationship (change in pressure is proportional to the square of the velocity). In this case, the device causing the change in pressure is not a sharp-edged orifice but rather a section of pipe that gently converges to a small diameter area (called a throat) before diverging back to the full pipe diameter. Figure 5.20 presents the operation of a typical venturi meter. 


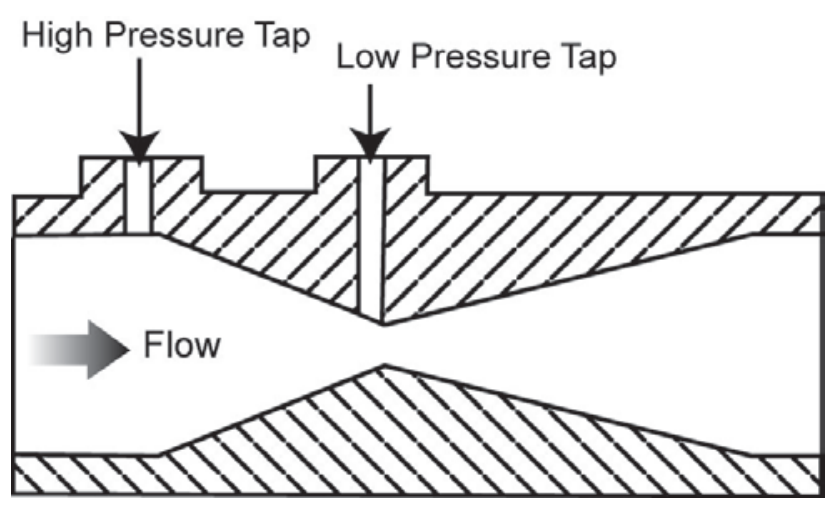

Figure 5.20. Typical Venturi Meter

The benefit of the venturi meter over the orifice meter lies in the reduced pressure loss experienced by the fluid. In situations where the cost to pump a fluid is high, this benefit can represent a significant savings over the life of the system. An additional benefit of the venture meter is its durability - it does not have the "sharp edge" profile of the orifice meter, therefore, it does not suffer from potential erosion issues and has a greater ability to accurately meter contaminated on non-homogeneous fluids.

The benefit of the venturi meter over the

orifice meter lies in the reduced pressure loss experienced by the fluid.

Venturi meters can be used on connection sizes ranging from 0.25 to 4 inches and have accuracies in the range of 0.25 to 2.0 percent, depending on fluid type and installation. Venturi meters can cost from $\$ 1,000$ to $\$ 5,000$.

Differential Pressure - Annubar Meter. The annubar flow meter (a variation of the simple pitot tube) also takes advantage of the velocitypressure relationship of flowing fluids. In this case, the device causing the change in pressure is a pipe inserted into the natural gas flow. Contained within this pipe are two smaller tubes with holes or ports evenly spaced along the length. Figure 5.21 presents the components of the annubar flow meter. When properly installed, one tube faces directly upstream and one downstream. These tubes and ports become the pressure detection points for the meter; the upstream-facing ports measure the flowing pressure (i.e., velocity pressure) and the downstream port measures the static pressure. Using these measured pressure values and the previously mentioned pressure-flow relationship, the flow rate is calculated.

Annubar flow meters have a turndown ratio of up to 10:1 and are relatively easy to install. These meters can make measurements on pipe sizes from 2 to 100 inches to an accuracy of 2 percent. Annubar meters can cost between $\$ 1,000$ and $\$ 3,000$, depending on size and accuracy.

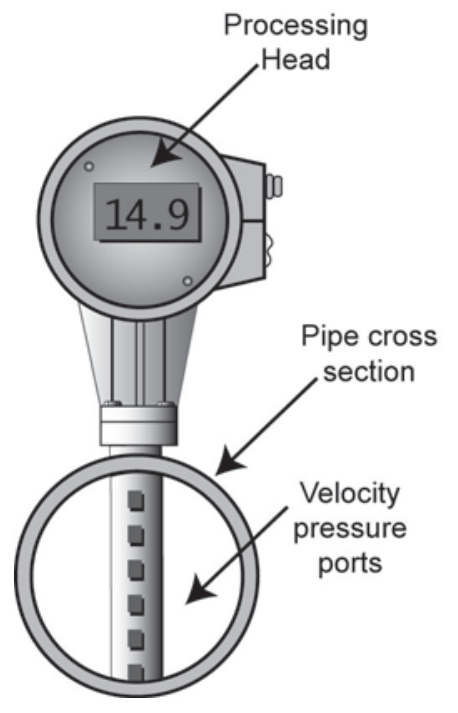

Figure 5.21. Typical Annubar Meter

\subsubsection{Velocity Meters}

Another means to determine fluid flow is by a technique that directly relates to the fluid's velocity. Velocity meters determine fluid flow by measuring a representation of the flow directly. Because the 


\section{Annubar Flow Meter Advantages/Challenges}

\section{Advantages:}

- Limited resistance to flow

- Good accuracy and repeatability

- Lower installation cost - particularly on larger pipe diameters

- Expanded turndown ratio.

\section{Challenges:}

- Installation needs proper lengths of pipe for accurate measurements. fluid's velocity is measured (i.e., not the squareroot relationship to determine velocity as with differential pressure meters), velocity meters can have better accuracy and usually have better turndown ratios than other meter types.

Velocity - Turbine Meter. Turbine meters operate as their name implies. A multi-blade impellor-like device is located in, and horizontal to, the fluid stream. As the fluid passes through the turbine blades, the impellor rotates at a speed related to the fluid's velocity. Blade speed can be sensed by a number of techniques including

magnetic pick-up, mechanical gears, and photocell. The pulses generated as a result of blade rotation are directly proportional to fluid velocity, and hence flow rate. Figure 5.22 details the components of a typical turbine meter. It should be noted that there are a variety of turbine designs and mounting configurations; Figure 5.22 highlights the concept.

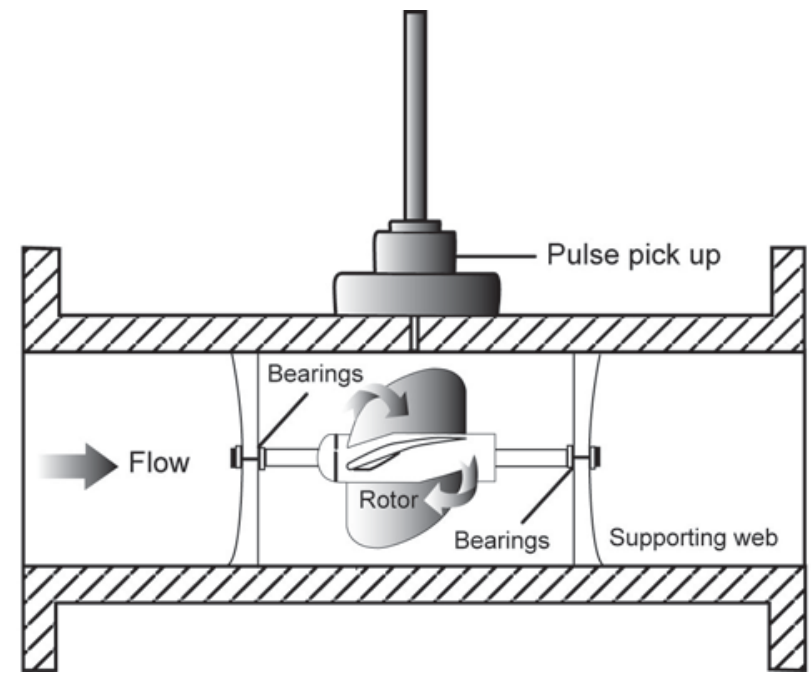

Figure 5.22. Typical Turbine Meter

Because turbine meters have mechanical elements in the fluid stream, they

Velocity meters can have better accuracy and usually have better turndown ratios than other meter types. meters can be susceptible to wear and resulting inaccuracies. Of particular concern is the bearings that, if exposed to corrosive or contaminated fluids, can quickly wear and result in inaccuracy. The high-temperature/chilled water applications for turbine meters are typically for larger industrial metering functions. Turbine meters are used on connection sizes ranging from 2 to 20 inches and have accuracies in the range of 0.5 to 1.0 percent, depending on fluid type and installation. Turbine meters can cost from $\$ 300$ to $\$ 2,300$, depending on size. 
Velocity - Vortex Shedding Meter. A vortex shedding meter senses flow disturbances around a stationary body (called a bluff body) positioned in the middle of the fluid stream. As fluid flows around the bluff body, eddies or vorticies are created downstream, the frequencies of these vorticies are directly proportional to the fluid velocity. Figure 5.23 presents the typical vortex shedding meter.

As the vorticies are developed along the bluff body, they grow and are detached - it is this detachment that is electronically sensed and totalized. Because the vortex shedding meter has no moving parts, it is a very reliable method of water measurement. As with the other non-positive displacement meters, the vortex shedding meter is used in very specific high-temperature hot water/chilled water applications; good examples are for boiler feed water metering or for chilled water flow metering. Vortex shedding meters can be used on connection sizes of 1 to 12 inches and has accuracies in the 1- to 2-percent range. Vortex shedding meters typically cost between $\$ 500$ and $\$ 3,000$.

Velocity - Ultrasonic Meters. Ultrasonic meters function through

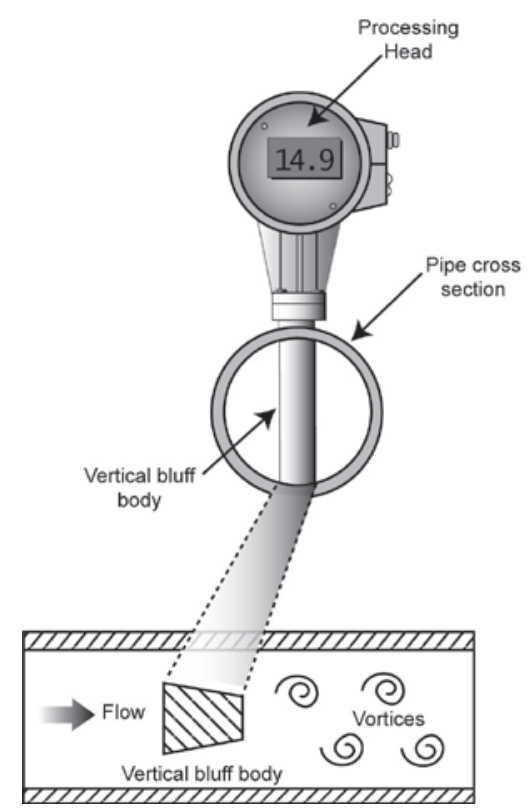

Figure 5.23. Typical Vortex Shedding Meter the basic relationship between the velocity of a fluid and its accompanying frequency shift - also known as the Doppler effect. One of the most attractive aspects of ultrasonic flow meters is that they are completely nonintrusive. That is, this technology does not require any permanent modifications or penetrations to piping or disruption of service for installation. All ultrasonic meters mount on the outside of the piping and can be used as both temporary and permanent metering.

There are two styles of ultrasonic flow meters available: Doppler meters and transit time meters. The Doppler meters function by measuring the frequency

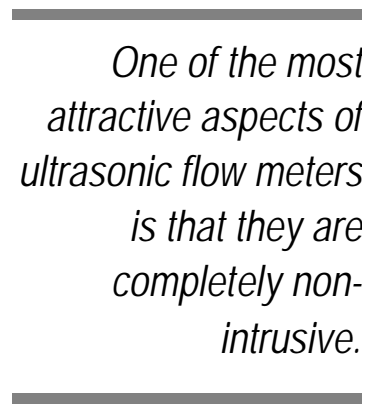
shift of the moving fluid to calculate flow. This technology works best when the fluid has suspended solids, bubbles, or other particles to reflect the ultrasonic signal. Figure 5.24 presents the function of the Doppler ultrasonic meter.

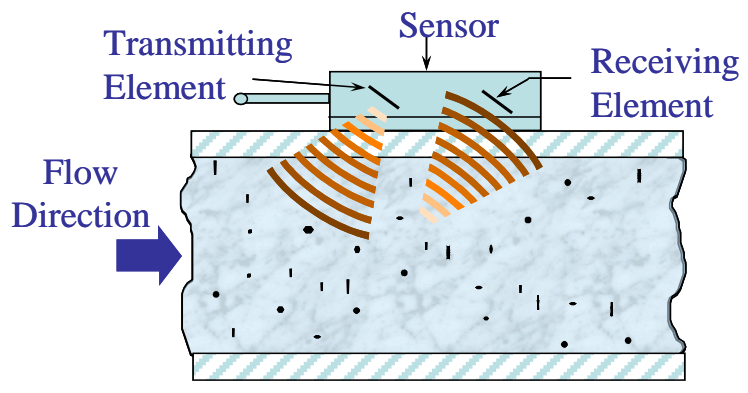

Figure 5.24. Doppler Ultrasonic Flow Meter 
The transit time meter makes use of a transmitter and receiver mounted on opposite sides of the pipe. As fluid moves through the system, the first transducer sends a signal and the second receives it. The time it takes for the signal to arrive is proportionate to the flow rate. Different from the Doppler meter, the transit time meter needs clean and viscous liquids for best accuracy. Figure 5.25 presents the function of the transit time ultrasonic meter.

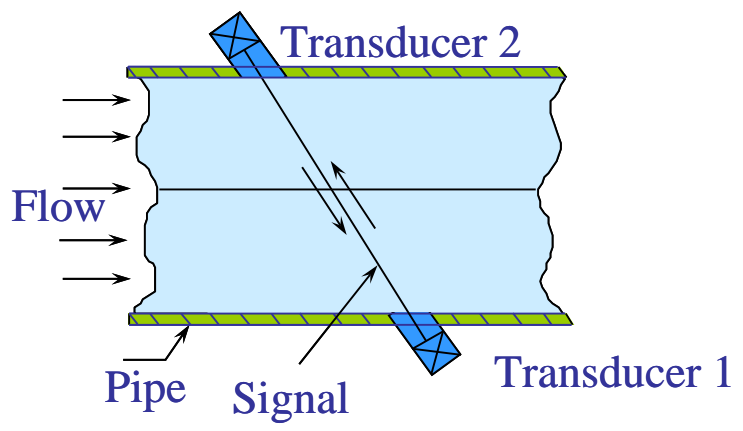

Figure 5.25. Transit Time Ultrasonic Flow Meter

Because ultrasonic meters have no moving parts, they are very reliable. These meters can be used on connection sizes up to 20 inches or larger, and have accuracies ranging from 1 to 5 percent. Both types of ultrasonic meters are relatively expensive, costing between $\$ 1,500$ and $\$ 6,500$.

\subsubsection{Recording Intervals}

Due to the way water is usually purchased and used, the data-recording interval is not as critical as it is for electricity. However, an ability to record water use at least hourly, if not every 15 minutes, can afford some diagnostic capabilities. One such capability would be to diagnose proper water flows and volumes based on known water uses. The best opportunity is to have the ability to modify the interval (at times to a shorter more refined interval) for temporary

Due to the way water is usually purchased and used, the datarecording interval for water is not as critical as it is for electricity. diagnostics and then return the setting to the longer more manageable interval.

\subsubsection{High-Temperature/Chilled Water Meter Maintenance}

Depending on the meter technology, installation, and quality of water delivered, water meters generally require limited maintenance. Procedures followed should be those as recommended by the manufacturer. Pending their availability, below are some general maintenance guidelines presented by meter technology.

\section{Positive Displacement Meter}

Monthly Inspections

- All connections for water leakage

- Noisy operation internal to meter

- Consistent and smooth register operation

- General meter cleanliness 
Annual Inspections

- Calibration according to manufacturer's recommendation or if trended data indicate miscalibration.

\section{Differential Pressure Meter}

Monthly Inspections

- All connections for water leakage

- Abnormally loud or discontinuous sounds internal to meter

- Check for properly connected and sealed pressure taps

- General meter cleanliness

Annual Inspections

- Check orifice diameter and edges for wear, roughness, or material buildup

- Check venturi for cleanliness and corrosion at throat. Clean and smooth all internal surfaces.

- Check for well-connected and sealed pressure taps

- Calibration of differential pressure sensors/transmitters according to manufacturer's recommendation or if trended data indicate miscalibration.

\section{Velocity Meter}

Monthly Inspections

- All connections for water leakage

While other outputs

- Abnormally loud or discontinuous sounds internal to meter options are available, calibrated pulses are

- General meter cleanliness

the most common

and relatively easy to

Annual Inspections work with.

- Impeller blades should be checked for wear or damage

- Impeller bearings should be checked for wear

- Calibration of velocity meter according to manufacturer's recommendation or if trended data indicate miscalibration.

\section{Ultrasonic Meter}

Monthly Inspections

- Transducer positions and attachment to piping

- Cleanliness of transducer/piping interface

- Verification of piping isolation (i.e., no vibration in metering section)

- General meter cleanliness 
Annual Inspections

- Calibration of meter according to manufacturer's recommendation or if trended data indicate miscalibration.

\subsubsection{High-Temperature/Chilled Water Metering Data Output/Communications Considerations}

Standardization on
communication
between meters and
other data acquisition
systems needs to be
considered.

While other outputs options are available (e.g., 4 to 20 milliamp, 0 to 5 volt, Modbus), calibrated pulses are the most common and easy to use. Chapter 6 addresses in more detail the different output and communications options.

When specifying the water flow meter, the pulse calibration is a critical parameter. Important in this specification is an understanding of the range of expected flow and necessary resolution of output. There are situations where too high of a pulse count (i.e., too high of a frequency) can result in saturation of the data logger or other collection device. A saturation condition usually results in loss of data and erroneous pulse counts; therefore, it needs to be avoided. Meter and data logger vendors should be consulted when determining the appropriate pulse count and calibration.

\subsubsection{High-Temperature/Chilled Water Meter Selection Considerations}

- Determine the temperature ranges the meter will operate in.

- Determine expected range of water flows and pipe sizes.

- Determine the accuracy requirements over the flow range.

- Identify any physical installation requirements for meter location, straight lengths of piping, available communications, etc.

- Communication interoperability - consider standardization on communication between meters and other data acquisition systems.

- Specification considerations - at the outset, consider a formal specification development so future additions ensure future compatibility.

When specifying the water flow meter, the pulse calibration is a critical parameter.
- Data processing - how will the collected data be processed? Does the metering equipment vendor offer this function/service? Do not overlook the effort it will take to create a process to collect, store, and archive the data.

- Facility staff buy-in - make certain those staff that will be installing, maintaining, and most importantly, using the data have a voice in meter selection. 


\subsubsection{High-Temperature Hot Water/Chilled Water Meter Selection Criteria}

Table 5.4 presents some of the more common water metering technologies and key criteria for selection decisions (FEMP 2010; Carbon Trust 2005).

Table 5.4. Common Water Metering Technologies and Key Criteria

\begin{tabular}{|l|c|c|c|c|c|c|}
\hline \multicolumn{1}{|c|}{ Criteria } & $\begin{array}{c}\text { Positive } \\
\text { Displacement }\end{array}$ & Orifice & Venturi & Turbine & $\begin{array}{c}\text { Vortex } \\
\text { Shedding }\end{array}$ & $\begin{array}{c}\text { Ultitasonic } \\
\text { Dop/TT }\end{array}$ \\
\hline Accuracy & Good & Moderate & Good & Good & Good & Moderate \\
\hline Turndown Ratio & $10: 1$ & $<5: 1$ & $<5: 1$ & $10: 1$ & $20: 1$ & $10: 1 / 20: 1$ \\
\hline Repeatability & Good & Good & Good & Low & Very good & Good \\
\hline Installation Ease & Easy & Easy & Moderate & Challenging & Moderate & Very easy \\
\hline Pressure Loss & Medium & Moderate & Low & Moderate & Low & None \\
\hline Recalibration Needs & Infrequent & Frequent & Infrequent & Frequent & Infrequent & Moderate \\
\hline Capital Cost & Low & Low & Moderate & Moderate & Moderate & High \\
\hline Installed Cost & Moderate & Low & Moderate & Moderate & Moderate & Low \\
\hline Maintenance Cost & Low & High & Moderate & Moderate & Low & Low \\
\hline
\end{tabular}

\subsubsection{References}

Carbon Trust. 2005. Good Practices Guide: Reducing Energy Consumption Costs by Steam Metering. Available at: http://thecesh.com/wp-content/uploads/2010/05/GPG018.pdf.

Federal Energy Management Program (FEMP). 2007. FEMP Metering Training Course Session 2: Metering Technologies, Communications, and Data Storage. April 4, 2005. Available at: http://eere.pnnl.gov/femp/metering_webcast.stm.

Federal Energy Management Program (FEMP). 2010. Operations \& Maintenance Best Practices: A Guide to Achieving Operational Efficiency, Release 3.0, prepared by Pacific Northwest National Laboratory for the Federal Energy Management Program, U.S. Department of Energy, Washington, D.C. Available at: http://www1.eere.energy.gov/femp/pdfs/omguide_complete.pdf. 
This page intentionally left blank. 


\subsection{Fluid Metering - Wastewater}

\subsubsection{Introduction}

Once potable water is used, it is typically discharged in a wastewater or effluent stream to a sewer system. The metering of these flows at the building level is very uncommon and given the cost and relative complexity, wastewater streams are usually metered only at points of distribution and treatment.

The two primary categories of wastewater metering are "open channel" and "closed conduit" flow measurements (EPA 2008). It should be noted that any considerations of metering wastewater flows need to be balanced with requisite health and safety precautions and follow all local code requirements.

\subsubsection{Metering Strategies}

\subsubsection{Open Channel Flow Measurement}

An open channel is defined as any open conduit (such as a channel or flume), or any closed conduit (such as a pipe) that is not flowing full. The most common methods of measurement for open channel wastewater flow include:

- Weir - defined as an overflow structure of specific geometry to allow for the calculation of flow. If the geometry of the weir is known, and all fluid flows over the weir, the depth of water behind the weir can be converted to a rate of flow. The construction of the weir and its orientation to the flow path are critical aspects to calculation accuracy.

- Flume - similar to a weir, flow calculations using a flume make use of the specific geometry of the device, the water level behind the structure, and flow constants specific to the design.

\subsubsection{Closed Conduit Flow Measurement}

Depending on the state of the fluid and its homogeneity, some of the same techniques for potable water can be employed for wastewater including the standard differential pressure techniques of orifice and venture meters. When accuracy or fluid properties preclude these standard techniques, other technologies can be employed, such as the following:

- Ultrasonic - the Doppler ultrasonic meter functions by measuring the frequency shift of the moving fluid (and suspended particles) to calculate flow. A primary benefit to ultrasonic metering is its nonintrusive nature. This system can be installed on the outside of most existing effluent piping.

- Electromagnetic - the electromagnetic flow meter functions according to Faraday's Law of Induction whereby a conductive fluid moving through an induced magnetic field results in a potential difference proportional to the fluid velocity. Electromagnetic flow metering is an intrusive technology requiring in-line installation.

The most common need to understand wastewater flow relates to sewer charges levied by water/ wastewater utilities. It is uncommon for utilities to assess wastewater charges based on a metered wastewater volume, rather these charges are estimated based on potable water use. 
It is standard practice for serving utilities to estimate annual wastewater volumes based on winter month's potable water use. This makes the assumption that most/all potable water delivered in these months is discharged to the sewer. While this assumption may be valid for residential water/wastewater use, it can be incorrect for facilities with water uses not discharging to the sewer system; examples of such systems include:

- Water lost in evaporation (e.g., cooling towers)

- Water used in a process or production (e.g., evaporative process loses or food production)

- Year-round irrigation use.

Most utilities are willing to consider these applications and allow some form of credit. However, it is usually incumbent on the user to provide approved equipment and the documentation to receive this credit. As discussed above, wastewater metering is an option; however, it is typically expensive and can be technically challenging.

An equally acceptable method to metering wastewater is simply metering the application that is not discharging to the sewer system. This can be done with standard potable water meters and usually at a fraction of the cost and effort of wastewater metering. For example, a cooling tower evaporates water to produce the necessary cooling effect for efficient chiller operation. The evaporated water, in many cases a significant quantity (see case study below), does not enter the effluent stream as discharge, therefore a credit should be requested. In most cases, because of the variable operation of cooling towers, water utilities will require installation of a metering point for proper allocation. Prior to meter installation, an agreement on the process, the meter/s, and data usage should be executed with the supplying utility.

\section{Case Study: Metering of Cooling Tower Make-up Water Proves Economic (BMP 2007)}

Typically, sewer charges for industrial sites are based on the percentage of cubic feet of water metered to a company's site regardless of its usage (e.g., drinking, cleaning, cooling). Water consumption charges are based on meter readings placed at an entry point to the site. Allowing for a small percentage for lawn watering, the Water Department calculates sewer charges from these same meter readings based on the assumption that water entering a site will exit the site through the sewer. In industrial sites such as Polaroid where film processing and machinery generate vast amounts of heat, large cooling towers are required to maintain stable temperatures and humidity levels. Although these cooling towers consume large amounts of water for operation, only about 10 percent of the water returns to the sewer system while the remaining 90 percent evaporates from the towers. As a result, Polaroid negotiated with the City of Waltham's Water Department for an annual rebate of sewer charges for the water which evaporates from its cooling towers.

Until 1996, Polaroid paid full sewer charges for the evaporated water. Based on widely accepted engineering practices, onsite evaluations, and cooling tower blow-down cycles, Polaroid confirmed that an average of 90 percent of the water consumption volume for its 16 cooling towers evaporates, and the remaining 10 percent is discharged into the sanitation sewer system through the blow-down cycles. This breakdown equates to a 10:1 reduction of water consumed versus water entering the sewer system. Key to qualifying for the annual rebate was Polaroid's presentation and demonstration to the city that the Water Department's metering and sewer charging practices were inadequately reflecting the actual water discharged to the sewer system. 
The city granted approval for the rebate, but required Polaroid to purchase and install new water meters at the intake of each of the water towers. The meters, which were compatible with the city's present metering system, registered in cubic feet and allowed for remote readout from a touchpad using a smart gun.

By using the new method to estimate sewer discharge, Polaroid established a reliable accounting method for determining how much water evaporates at the cooling towers and how much enters the sewer system. Sewer charges are no longer based on the assumption that all water entering a site will be discharged through the sewer system. Polaroid's annual sewer charge rebate for its 16 cooling towers is estimated at $\$ 150,000$ to $\$ 200,000$ with a hardware implementation cost of less than $\$ 4,000$.

\subsubsection{References}

Best Manufacturing Practices Center of Excellence (BMP). 2007. Best Manufacturing Practices 2007. Best Practice: Cooling Tower Make-Up Water Metering. College Park, Maryland. Available at: http://www.bmpcoe.org/bestpractices/internal/polar/polar_15.html.

U.S. Environmental Protection Agency (EPA). 2008. U.S. Environmental Protection Agency Wastewater Flow Measurement. Scientific and Ecosystem Support Division (SESD) Operating Procedure - Wastewater Flow Measurement. SESD PROC-109-R2. 
This page intentionally left blank. 


\subsection{Metering of Onsite Power Generation}

Onsite power generation can take a variety of forms, from the traditional combustion-turbine (including combined heat and power [CHP]) to more recent developments in fuel cells, photovoltaics, wind, methane gas, and geothermal electricity generation, to name a few. While the method of generation may vary, the need to understand the amount, timing, and duration of the power generated is consistent. With the advent of the smart grid and various incentives targeting onsite and renewable power generation, there has been an increase in interest, opportunity, and regulation of these systems.

To fully understand metering needs and considerations, a few definitions are required:

- Net Metering: A utility procedure for crediting customers for electricity generated onsite and in excess of their own needs. In its simplest form, this allows for the flow of electricity both to and from a customer's location through a single bi-directional meter (IREC 2009).

- Interconnection: The physical connection of the customer's generation source to a utility's distribution network. Implicit in this are the requirements and protocols for code compliance and safety standards.

- Islanding: The condition in which onsite power is still being generated while the grid is down. This situation creates a variety of safety and equipment concerns and is usually a stipulated code issue. Many modern onsite power systems have (as are required) anti-islanding mechanisms that cease to energize upon detection of line voltage/frequency anomalies.

\subsubsection{Metering Strategies}

The metering of onsite power equipment will vary with configuration, monitoring objectives, and system type. A few of the more common power-generation scenarios are presented below.

Traditional Combustion - typical fossil-fuel fired power generation is often used as back-up generation and/or for peak load (electric) management or utility-sponsored demand response programs. Metering of time series electricity generated ( $\mathrm{kW}$ and $\mathrm{kWh}$ ) along with fuel use is recommended.

Combined Heat and Power (CHP) - in situations where a fossil fuel is used to generate heat for both power generation and process/conditioning applications, metering is recommended at multiple points. Included in these points are the following:

- Fuel - quantity of fuel used (volume and rate) in a time-series record

- Electricity - time dependent power generated, net of process, and at relevant intervals (typically 15 minute) to assure proper credit for utility program participation/incentive

- Heat - quantity of useful heat generated over the time interval.

Renewable Energy - solar thermal, photovoltaic, ocean, and wind are resources requiring single-point metering. These generation assets should be metered at the point of generation (electric) or for multiple systems at the aggregation point. A typical PV line diagram is shown in Figure 5.26. Note the presence and location of disconnects and the production meter. 


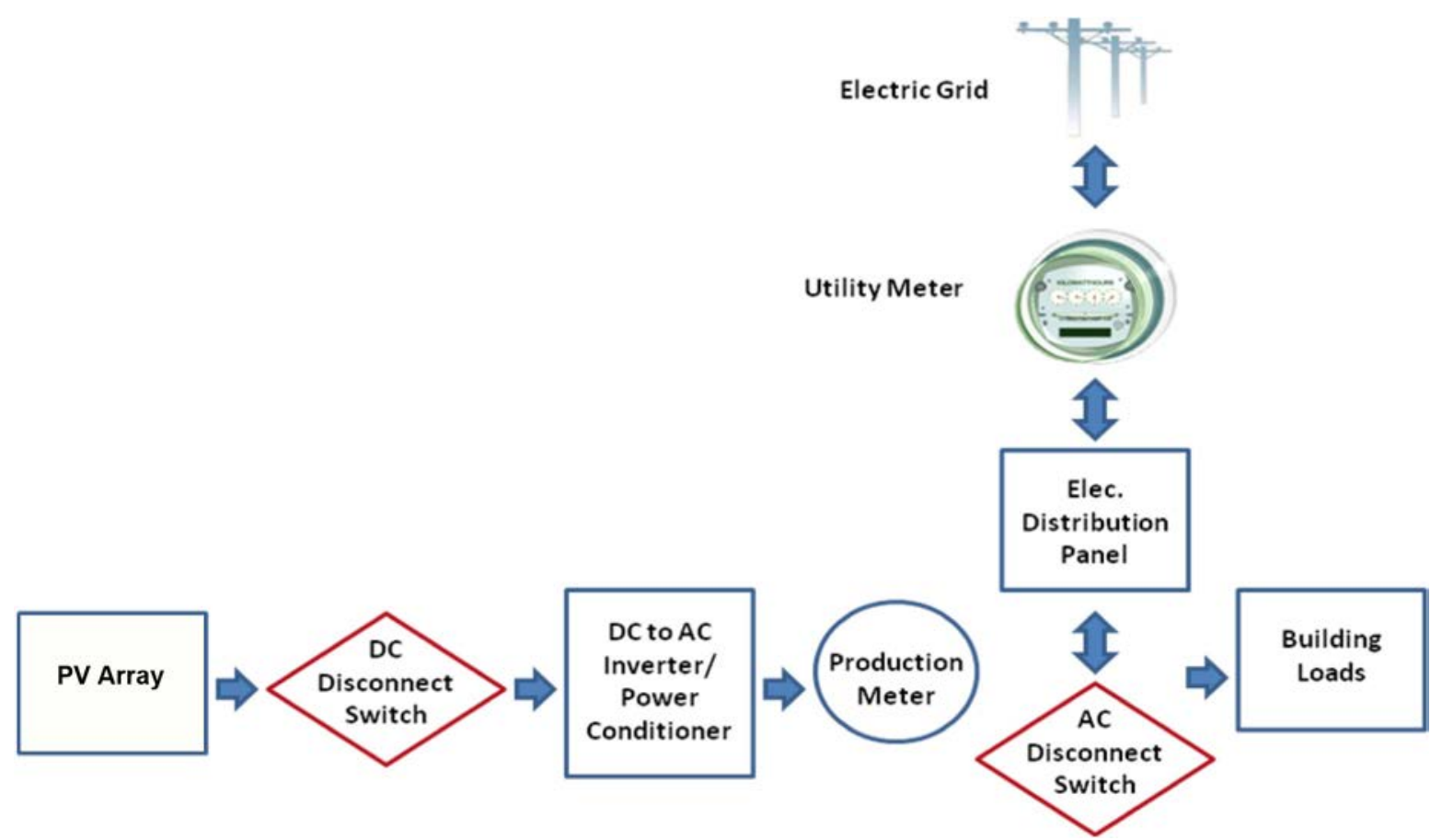

Figure 5.26. Typical Photovoltaic Array Connection and Metering Scheme

Fuel Cells - similar to CHP and depending on application, fuel cell metering is recommended at multiple points, including:

- Fuel - quantity of fuel used (volume and rate) in a time-series record

- Electricity - time dependent power generated, net of process, and at relevant intervals (typically 15 minutes) to assure proper credit for utility program participation/incentive

- Heat - quantity of useful heat generated over the time interval.

\subsubsection{Metering Applications}

The use of metered data for onsite power monitoring will vary with the application of the power. A few of the more common applications of these data are presented below:

Shadow metering - quantify and verify the system generation and assure proper "net metering” credit.

System performance - metered data become very useful in confirming operational parameters of system availability and efficiency.

System maintenance - data are valuable to schedule maintenance (run-time based) as well as to trend and predict system degradation.

Performance contract verification - if the system was installed as part of a performance contract, these data will be useful in power generation and efficiency validation and for verification of proper contractor payment. 


\subsubsection{Safety, Codes, and Regulations}

Whether a system is functioning in a net-metered configuration with full grid interconnection or operates as a stand-alone remote system, safety is paramount. Any system connecting to the grid in any manner will require "anti-islanding” capabilities. This safety measure prevents power generation and transmission onto the grid during outage periods. This measure is critical to prevent injury to utility staff working on an otherwise non-electrified portion of the grid.

Beyond anti-islanding measures, onsite power generation and accompanying metering should be in compliance with all local electrical and safety codes. While many of the technical issues related to interconnection and metering of onsite power generation have been addressed through the development of national standards such as the Institute of Electrical and Electronic Engineers (IEEE) 1547 Standard for Interconnecting Distributed Resources with Electric Power Systems (IEEE 2003), there are at times variances with local codes that need to be explored.

\subsubsection{References}

Institute of Electrical and Electronic Engineers (IEEE). 2003. IEEE 1547 Standard for Interconnecting Distributed Resources with Electric Power Systems. Approved June 2003. IEEE, Washington, D.C. Available at: http://grouper.ieee.org/groups/scc21/1547/1547_index.html.

Interstate Renewable Energy Council (IREC). 2009. Connecting to the Grid, A Guide to Distributed Generation and Interconnection Issues. $6^{\text {th }}$ Edition, Interstate Renewable Energy Council, Washington, D.C. Available at: http://www.irecusa.org/wpcontent/uploads/2009/10/Connecting_to_the_Grid_Guide_6th_edition-1.pdf. 
This page intentionally left blank. 


\section{Chapter 6 Metering Communications and Data Storage}

An integral part of the overall metering system is the mode of communications from the sensors to the meter and then from the meter to the point of data storage, analysis, and archiving. The communication from sensor to meter is usually handled internal to the meter and largely transparent to the user. The communication from the meter to the ultimate storage, analysis, and archiving is the focus of this chapter.

Regardless of the meter type, once data are collected they need a communication pathway to a location where the data will be processed, stored, and used. This pathway should be amenable to the various meter output types - some of the more common output types include:

- Analog output - typically, 4 to $20 \mathrm{~mA}$ or 0 to 5 volts de

- Contact closure - pulse type output

- Digital output - digital pulse

- Digital signal - outputs using networked communications (e.g., Ethernet, Modbus, HART).

Many of the newer digital-signal output meters can output multiple signal types offering a variety of communications options. These meters can be serially addressed, affording a lowered installed cost through reduced wiring installation and expense (i.e., multiple meters communicating on one pair of wires back to the data-collection terminal). Often these outputs can be viewed on local displays integral to the meter. These displays are quite useful for field set-up, calibration, verification of function, and troubleshooting. Developments in wireless technologies have resulted in lower meter cost and improved ease of

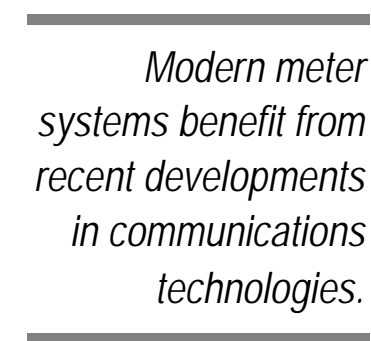
installation.

\subsection{Traditional Communications Options - Non-Automated}

Modern meter systems benefit from recent developments in communications technologies. Over the past 20 years, communications have moved from requiring a hand-written recording of the metered value to a manually entered electronic recording to a locally transmitted electronic value. These data collection/ communications modes are still in practice and are described below.

- Sneaker-net Data Collection. A largely outdated, yet still practiced, method of manual meter reading involving writing down or keying in to a hand recorder the metered data. This data collection practice is inefficient, inaccurate, and discouraged in most applications.

- Mobile-Radio Data Collection. This technology makes use of close-proximity radio frequency (RF) communications where by data are transmitted by the meter to a receiver - usually located in a slowly moving vehicle. While more accurate than sneaker-net, it still has an in-field manual collection component - driver and vehicle.

With the enactment of EPAct 2005, which explicitly states that metered data will be collected automatically, via automated meter reading (AMR) and made available at least daily, Federal agencies are now required to use AMR - where practicable. As such, the following section presents the AMR systems that 
are more common and applicable to the Federal sector. Section 2.3 highlights the various legislative requirements and agency directives related to metering and communications.

\section{Phone Modem}

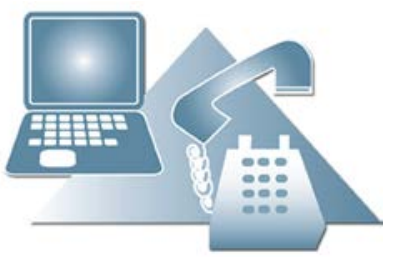

Advantages:

- Proven technology

- A secure and private network

- Usually available

Challenges:

- Can be expensive

- No access to real-time data

- Wired installation (hardwire solution) using additional equipment (modem)

\section{Local Area Network}

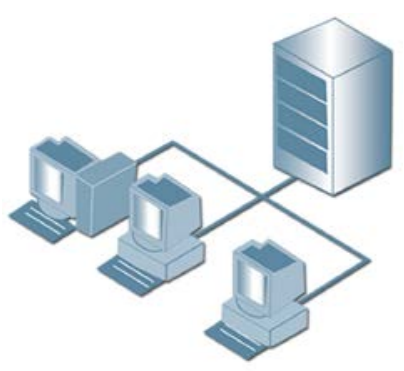

Advantages:

- Proven technology

- Increasing availability

- Always connected

- Data sharing opportunities

Challenges:

- Network/IT security concerns

- Wired installation - need for network connectivity

\subsection{Modern Metering Communications - Automated Meter Reading (AMR)}

AMR systems, both wired and wireless, are increasingly being used because of their availability, reliability, and decreasing cost. Many utilities, large corporate campuses, and universities are finding AMR not only to be convenient, but also to make good business sense. When developing the communications portion of your metering program, it is important to consider what existing communications infrastructure you can take advantage of (e.g., building automation system, local area network) to potentially lower the cost of AMR. In addition, if you have a large site with distributed buildings, you may find benefit in considering multiple communications technologies (e.g., networks in one area, phone lines in another, and wireless in a third) to gain the necessary communications coverage.

Below are the predominant AMR technologies along with some of the benefits and challenges of each.

Phone Modem. Taking advantage of telephone modem technology in both hardwire and wireless (i.e., cellular), this communications solution is the oldest and traditionally most reliable of the technologies. In typical applications, automated software (usually from the metering equipment vendor) is used to dial (phone-in) the modem daily to retrieve accumulated data. In addition to the phonein systems, there are meters that can phone-out at preset times or at specified data accumulation levels. It should be noted that phone lines do not have to be dedicated to the meter(s) they serve; there is no reason meters cannot share a phone line with other applications even personal office phones. Shared phone line applications can use off-hours for data communications and, therefore, do not interfere with other business-related uses.

Local Area Network. Using an existing building or site's computer network to serve as the communications path for the metering system can be very economic. When properly configured, meters can communicate over this network using a variety of open protocols, including Modbus, HART, transmission control protocol/internet protocol (TCP/IP), BACnet, etc. In addition, these meters usually can be serially addressed and linked together (daisy-chained) to minimize wiring installation and expense. 
Beyond the local area network (LAN), wide-area networks (WANs) can be developed by linking more than one of these networks together. This becomes useful to large sites with many distributed buildings and locations. Additional benefits to the LAN solution include the ability to share data throughout the network and view data in real time. Prevalent concerns with using a LAN for data communications stem from perceived security issues with transferring data over secure networks and potential access to the LAN via the metering points. In both cases, these concerns can be addressed with typical LAN security protocols. As is often the case, some level of education on the system, its operation, and security may be necessary - a small demonstration of the system may help convince skeptical IT or informationsecurity staff.

Building Automation System. By using an existing building automation system (BAS), we again take advantage of a site's previous investment in existing infrastructure. In this case, the wiring used for BAS communication becomes the metering communications path. In this case, the meters are treated as other "points" on the BAS and function much as other sensors or points on the system (i.e., communicate to and from the central host computer). The BAS is a workable solution only when there is excess capacity to add points and system software is capable of using the meter's data output protocol - both of these factors need be verified with the BAS and metering equipment vendors. An additional constraint to the BAS solution relates to the host computer's ability to allocate memory for these data and offer an ability to retrieve data sets in an automated fashion.

Radio Frequency/Wireless Networks. Becoming increasingly available and economic, wireless radio frequency (RF) communications makes use of wireless transmitters and receivers to communicate metered data. Wireless communication (FEMP 2007) offers the benefits of lowered installation cost, flexibility in metering locations, and minimizes disruption in service when compared to other options. Some of the limitations to wireless communications include the effective distance of communication (typically less than 300 feet) and the building's materials of construction that may impede or block the RF signal. Both situations can be mitigated by using a repeater or mesh network configuration. Similar to the LAN solution, wireless communications has perceived challenges including security issues and potential for interference with other sensitive communications equipment. In many cases these concerns are unfounded, yet some level of education on the system, its operation, and security may be

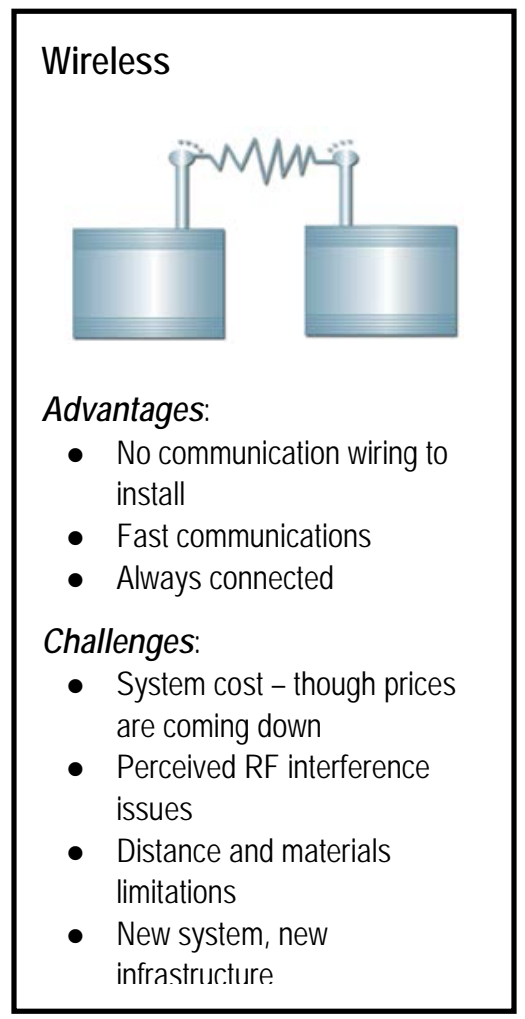




\section{Power Line Carrier}

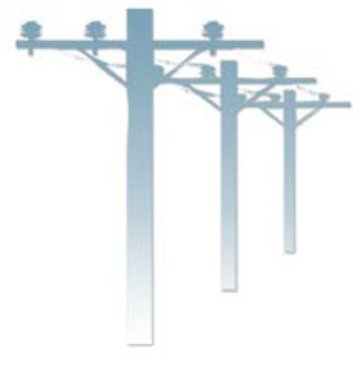

Advantages:

- Uses existing infrastructure

- Always connected

Challenges:

- Speed of data transfer

- Technical issues with transferring data past transformers necessary - a small demonstration of the system may help convince skeptical IT or information-security staff.

Power Line Carrier. This technology uses existing electrical wiring, both internal and external to buildings, as the communications conduit. While making use of the existing infrastructure gives this technology and economic advantage, limitations related to speed and quantity of data transfer and the ability to transfer data across standard electrical transformers can occur. Organizations making productive use of this technology, notably utilities and sites with many distributed buildings, do so by spreading the considerable installed cost over many metering points, or utilizing available communications technology that overcome this barrier.

As noted in the data output/communications considerations sections under each of the metering technologies in Chapter 5, many meters offer more than one communication output option. Most meters are a pulse or other protocol (e.g., Bacnet, Lonworks, or Modbus), whereby these data are either collected at the meter for future upload or sent (pushed by meter/pulled by collector) in real-time to the data collection point.

\subsection{Data Storage Software}

Assuming proper data collection and communication, some form of data storage system will be needed. Data storage needs will depend on the number of meters connected, the number of parameters metered, the data interval, and the expected need for access to historic data. In most cases, one of any number of commercially available database software systems will function well for data storage and software interface.

The specific requirements of the data storage/database system should be decided with assistance from site IT staff or others knowledgeable, or those who will be using the system. Below are draft specifications based on work done for the California Energy Commission Public Interest Energy Research Program (PIER) and the Building Technologies Program of the U.S. Department of Energy (CEC 2007).

\subsubsection{Data Storage Specification Considerations}

Sufficient data storage capacity will be able to store at least two years of data for all data points. database format or time series format. Minimum requirements are a SQL server or equivalent.

- The database shall allow other application programs to read and access the data with appropriate password protection while the database is running. The database shall not require shutting down in order to access or have data added. 
- Trend, or interval, data shall be archived in a database from field equipment in time intervals no less than once per day.

- Storage on the field equipment will be reset once data are exported to allow for trending if communication is disrupted. Data will be uploaded once communication is re-established.

- Blank or null values in the database will be replaced with actual data. Calculations and other metrics will be updated once controller data are uploaded. This overall system update to check for new data should be automated to run once a day.

- All data shall be stored in database file format for direct use by third-party application programs (e.g., an energy information system [EIS] as discussed in Chapter 7).

- Sufficient data storage capacity will be able to store at least two years of data for all data points. In addition, storage capacity will also allow for compression of one year of data for historic trends and archiving.

- Time stamps shall be collected on all data. The time stamp, depending on system architecture, will be captured at the field controller or system controller and directed to the database archive.

- Exported data shall contain no duplicate records or duplicate time stamps in output files. Each date/time stamp for a specific point shall be unique. The export query shall be for a specific point or multiple points in a defined group.

- Date/Time fields shall be in a single column in a format automatically recognized by common spreadsheet, database software tools, or EIS.

- The data shall be fully contained in a single file or table for each point. Data shall not span multiple files or database tables. Users can have the option to modify export file start and end file date span depending on third-party program requirements to evaluate the data.

Key to productive use of data is the access for analysis, whether done in-house or as part of a third-party EIS package or agreement - see Chapter 7 for more details.

\subsection{Data Storage Hardware}

The computer hardware for data storage and software execution should have ample processing power and memory to run the chosen database system and to process, store, and archive all collected data. Fortunately, such activities can be handled quite easily with modern stand-alone personal computers and/or workstations. One key recommendation is that whatever system is chosen, it be dedicated to this activity. It is also recommended that this system have an automated back-up function (typically daily) to a separate system/server for data protection and archiving.

The computer hardware for data storage and software execution should have ample processing power and memory to run the chosen database

system and to process, store, and archive all collected 
An increasingly popular option for data storage involves an agreement with a third-party organization (e.g., an EIS vendor or other data-hosting entity) whereby all data are collected, stored, and backed-up on vendor computer servers. In this case, the client is given access, usually over the web, to all data and analyses for addition processing, reporting, and downloading.

\subsection{References}

California Energy Commission (CEC). 2007. Continuous Performance Monitoring Systems, Specification Guide for Performance Monitoring Systems. Available at: http://cbs.lbl.gov/performancemonitoring/specifications.

Energy Policy Act of 2005 (EPAct 2005). 2005. Public Law 109-58, as amended, Section 103, Energy Use Measurement and Accountability, Section 543 (42 USC 8253), (e) Metering of Energy Use.

Energy Independence and Security Act of 2007 (EISA 2007). 2007. Public Law 110-140, as amended, Section 434(b).

Federal Energy Management Program (FEMP). 2007. Wireless Temperature Sensors for Improved HVAC Control. DOE/EE-0319, U.S. Department of Energy, Energy Efficiency and Renewable Energy, Washington, D.C. Available at: http://www1.eere.energy.gov/femp/pdfs/tir_wirelesstempsensors.pdf. 


\section{Chapter 7 Data Analysis and Use}

\subsection{Introduction}

Depending on the interval and collection frequency, metered data can accumulate quickly and become overwhelming unless some level of automated data processing is implemented. At the outset of any metering activity, it should be made clear that meters provide data and these data generally do not constitute information or knowledge. The reality of data analysis comes in the recognition that data need to be processed to create information (knowledge) before any proactive actions can be taken. To be successful in its metering activities, the Federal sector must recognize that meters and data alone are not the answer; success comes when the data are processed to create information and this information is used for action.

This chapter focuses on suggestions for productive uses for metered utility data as well as discusses some of the options for data analysis and processing.

\subsubsection{Data Analysis and Energy Information Systems}

An energy information system (EIS) is an integrated development combining analytical software and hardware (sensors and meters) with a communications system to collect, analyze, and report building energy/resource data. While these systems can be installed in a "turnkey" sense (i.e., all sensors, meters, and software are installed), many applications make use of existing hardware and communications and the EIS becomes strictly a software solution. The balance of this section will focus on the software data analysis aspects of the EIS

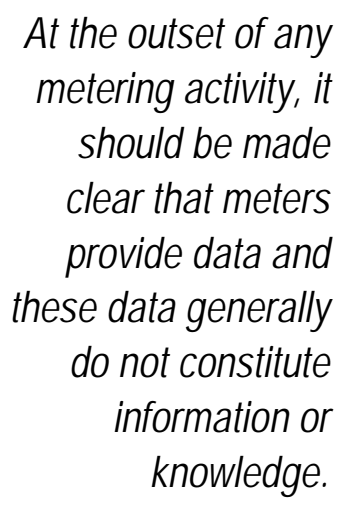

At the outset of any metering activity, it clear that meters provide data and se data generally not constitute knowledge. solution.

The software aspect of the EIS can take the form of a very simple (in-house developed) spreadsheet-based routine for processing metered data to a very complex (third-party purchased/licensed) software package with many sophisticated routines and statistical analysis capabilities.

As with the different offerings, there is a variety of fee arrangements available through these vendors. Some EIS organizations offer their service under a licensing agreement allowing only a certain number of users, or "seats." Other EIS organizations offer unlimited users under a time-based subscription service, and still others offer their product as a one-time software purchase with fee-based technical support. A list of EIS vendors can be found in the following references: CEC (2007) and LBNL (2002). A recent work by Granderson et al. (2009) provides information regarding the current state of the technology of available EIS systems, the distinguishing capabilities, and their functionality. The same report provides case study information to help understanding the interplay between common features, diagnostics, and energy-saving actions. 
EIS Development/Selection Considerations. Prior to making the decision to develop an in-house EIS versus purchasing/licensing a third-party product (or something in between), your organization needs to determine the options and expectations of the EIS in at least the following areas (CIPEC 2004):

- Definition of facility objectives - need to be clear on how the EIS will be used, how it will improve resource efficiency, and its contribution to the financial payback of the overall metering system.

- EIS integration with other IT systems - an important consideration prior to EIS selection/development is what existing infrastructure can be used. Prior to the EIS decision, a survey of existing facility software, hardware, communications, meters, and capabilities is recommended.

- Identify key reporting outputs - how will the outputs of this system be used to fulfill the objectives of the facility and its management? How will the outputs be made available (paper copy, email reporting, web-based reporting) and what sort of summary options are available?

- Data-collection needs - what are the data-collection requirements necessary to achieve the key reporting outputs? Are these available with existing metering elements?

- Data analytics - what analysis, statistical and/or regression routines are needed to transform the data into the information desired? Are the chosen analytics capable of handling the size, frequency, and complexity of anticipated data?

- Management support - has a budget been developed addressing expenses such as ongoing training, periodic testing, technical assistance, and troubleshooting?

EIS Vendor Selection Criteria. If you decide to use a vendor-based EIS system before selecting an EIS vendor, you may consider developing some vendor-selection criteria specific to your site. Below are some criteria developed as part of an EIS demonstration in California (LBNL 2006). While the criteria listed below may not align with your specific needs, it is highly recommended that you develop specific criteria well before you engage with EIS vendors.

Before selecting an energy information system vendor, you may consider developing some vendor-selection criteria specific to your site. The following list presents the questions to consider when evaluating each vendor during the selection process.

\section{Vendor Profile:}

- Does the vendor have experience and a stable business history in the particular EIS-related services?

- Does the vendor have experience working with industrial customers?

- Is the vendor capable of single source responsibility?

- Does the vendor have a regional office close by or in your state?

\section{Metering:}

- What type of data is the EIS designed to monitor/archive? Although most of the EIS products can be customized to archive any kind of data, some EISs may focus their functionalities on specific data types such as whole-building electricity, gas, or other utilities. If you are planning to perform more detailed diagnoses, built-in analysis functions for electric sub-meter, pressure, or temperature data will be helpful. Calculated data points and the capability to create a virtual data point from real measurement point values are also helpful. 
- What is the system response speed? In general, communication response speed for an industrial-grade system is 200 to 500 milliseconds or less, and commercial grade is 1 to 2 seconds. For only monitoring purposes, a slower response speed is acceptable, while industrial control requires faster response speed than commercial.

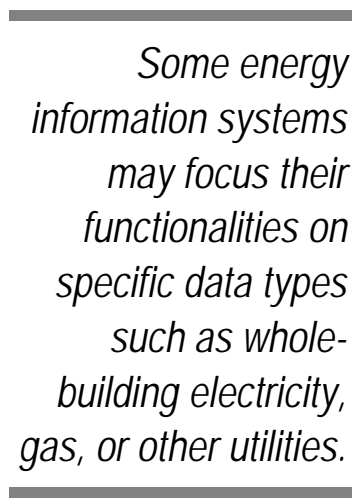

- What types of data input are supported by the EIS? Types include pulse, 0 to 10 volt dc, 4 to 20 milliamp, digital, etc.

- What types of metering/communication protocol are supported by the EIS? Types include TCP/IP, BACnet, LonTalk, Modbus, Profibus, etc.

- If your facility uses some specific protocol or has some native equipment for the specific protocol, compatibility between the system and the EIS will enhance its performance.

\section{Data Storage:}

- What is the database compatibility for the EIS? Is it compatible with one of the following common systems:

- Web-service client with extensible markup language (XML)

- Open database connectivity (ODBC) compliant to interface third-party software application

- Structured query language (SQL)

- Application program interface (API) to communicate with specific field devises such as handheld equipment

- Is the database and the data transfer through the Internet encrypted?

Data Output Considerations. The final element for consideration in EIS development or selection is the output information. Prior to development or selection, you need to review the objectives and goals of your entire metering system. Once identified, these can be used to help shape the type and form of EIS output necessary to achieve these objectives. Assembled below is a variety EIS output options collected from different vendors and resources. These are grouped into the categories of graphical outputs, analytical outputs, system-specific outputs, and utility outputs. While these categories are not allinclusive, they should provide some guidance in identifying the capabilities and outputs advantageous to you and your systems. One additional caution, as you make your decisions on vendors and levels of service/outputs, keep in mind the associated volume of data and the necessary time to process and act on the data. The recommendation is to start with a manageable set of outputs that can be useful in the expected time allocated. Then, as the system becomes more integrated, look to add features and other options. To start - keep it simple.

Graphical Outputs: Consider these as the plots you and your staff interact with on a daily, weekly, and monthly basis. Make certain axes are scaled and labeled for intuitive understanding now and into the future. 
- Daily profile: Time-series daily load profiles are displayed with time, in intervals of an hour or less, along the horizontal axis and load along the vertical axis.

- Day overlay: Overlay plots display multiple daily profiles on a single 24-hour time-series graph.

- Multi-point overlay: Allows viewing of multiple time series data points on the same graph.

- 3-D surface chart: Three-dimensional surface charts often display the time of day, date, and variable for study.

- Calendar profile: View up to an entire month of consumption profiles on a single screen as one long time series.

- X-Y scatter plots: X-Y scatter plots are useful for visualizing correlations between two variables.

Analytical Outputs: Consider which analyses will be most useful and incorporate those into the EIS as an automated function. The goal is to minimize the amount of exporting and re-analyses needed.

- Basic statistical analysis: Perform statistical calculation, such as mean, median, standard deviation, correlation, and regression.

- Benchmarking: Benchmark against building energy standards or public database such as EnergyStar.

- Intra/inter-facility comparisons: Benchmark against the building's historical data or across multiple buildings in the enterprise.

- Aggregation: Aggregate data among multiple data points. Integrate different energy units using energy conversions (e.g., kWh, Therm, etc., into Btu).

Consider which

analyses will be most

useful and

incorporate those

into the energy information system

as an automated

function.
- Data mining (data slice/drill-down): Sum-up/drill-down time series data by monthly, weekly, daily, hourly, or trended interval.

- Normalization: Normalize energy usage or demand by some factors such as building area, number of occupants, outside air temperature, and cooling or heating degree-days (CDD, HDD) to make a fair comparison between buildings.

- Hierarchical summary: Summarize usage and cost information by different levels. For example, starting from equipment energy cost, individual building energy cost, site energy cost, to regional energy cost.

System-Specific Outputs: Look for customized analyses beyond the "graphical and analytical" outputs mentioned above. This type of analysis often takes multiple data points and use more complicated algorithms.

- Power quality analysis: Monitor the voltage or current phases for conditions that could have adverse affect on electrical equipment.

- Steam charts: Calculate temperature, pressure, specific volume, and enthalpy for saturated steam and water.

- Forecasting: Forecast future trends by historical data and related parameters. 
- Validation, editing, estimation: A process performed to ensure quantities (kWh, $\mathrm{kW}, \mathrm{kVar}$, etc.) retrieved from meters are correct. The process includes validation of data within acceptable error tolerances, editing or correcting erroneous data, and estimating missing data.

- Equipment fault detection and diagnostics: Diagnose equipment failure or degradation based on customized algorithms and parameters.

Utility Outputs: Consider how these data can be useful in interactions with your utilities. Typically, there are tools allowing rate comparisons, bill verification, etc.

- Invoice verification (bill validation): Utility bills are compared to meter readings (so-called "shadow" metering) to validate accuracy of bills.

- Energy cost drilldown: Using energy tariff and usage data, calculate daily or hourly energy cost breakdown, instead of the usual cost that can only be seen in monthly utility bills.

- Real-time cost tracking: Calculates electricity costs every day or hour using real-time meter reading and rate tariffs.

- End-use cost allocation: According to user-defined parameters and algorithms, estimates end-use energy consumption from whole-building energy. Generally used for cost allocation to building tenants. A common parameter definition is energy use per square foot.

\subsection{Uses for Data - Metering Applications}

Given that some level of data analysis and processing is enacted, there are a variety of potential applications for these data. Note that while all of these applications may not be applicable to a given facility currently, future considerations should be part of the planning process - as such, some of the more common applications of metered data are presented below.

Reimbursable Billing. One of the most common applications of metered data is for accurate billing of site tenants based on actual use instead of estimates based on square footage or occupancy. Federal sites with tenants (or Federal tenants on private sites) are well advised to consider installing utility metering (gas, electric, water, steam) to not only provide accurate accountability of

One of the most common applications of metered data is for accurate billing of site tenants based on actual use instead of estimates based on square footage or occupancy. utility usage, but also send the tenant the correct price signal as an encouragement to save energy and other resources.

Validation/Planning/Reporting. Utility meters offer the facility staff the ability to validate existing metering ("shadow" metering) while allowing verification of usage and proper billing. While this activity may appear to have a minimal potential for savings, numerous cases of utility company errors are reported every year - and many of these result in large dollar value corrections, many in favor of the customer. Beyond validation, utility metering allows site staff to accurately comply with Federal reporting requirements and offer better information for systems and site planning activities.

Utilities Interaction. In many parts of the country, electric utilities are offering a variety of creative ratebased products targeting a higher reliability of the electrical grid. Participation in most of these programs usually requires some level of advanced metering; sometimes this is provided by the utility, other times it 
is the requirement of the site. In either case, having access to advanced-metering data (usually 15-minute interval demand data) will allow a site to best understand its particular load characteristics when negotiating with prospective electric utilities and their offerings. A few of the more common utility offerings are described below:

- Time-of-use pricing. These programs are designed to incentivize the "off-peak" use of electricity by offering reduced $\mathrm{kW} / \mathrm{kWh}$ charges during pre-defined and fixed off-peak time periods. Electric meters with interval capability (at least hourly intervals) allow the user to understand the value of these programs to their specific electricity use characteristics. In addition, the data from these meters allow for scenario planning activities whereby the value of shifting loads to off-peak periods can be estimated.

- Real-time pricing. Similar to time-of-use pricing, real-time pricing encourages decreased energy use during peak utility periods. However, rather than having predetermined fixed periods and associated energy costs, real-time pricing allows the utility to vary both-and does so giving the customer a nominal notification period. Sometimes these notifications are "day-ahead" or as short as "hourahead" notification. To take advantage of these offerings, facility managers need to have real-time metering and the flexibility to curtail loads commensurate with the utility needs and curtailment periods.

- Load Aggregation. Agencies may want to combine facilities that are geographically separate from each other for purposes of acquiring and billing utility services. Such aggregation, depending on the base load and peak load characteristics, can result in lower utility rates rather than a separate utility account and the associated "fixed" charges for each site.

Efficiency Opportunity Identification. Metering provides the data to begin validating equipment performance and efficiency. As previously described in Chapter 4, the metering hierarchy of starting with whole-building metered data (preferably 15-minute interval data) and looking for anomalies or unexplained events/usage is recommended. Once identified, these events become candidates for further analysis and trending, or as points for a more detailed level of metering using portable data

\section{Energy Policy Act of 2005, Section 1252 "Smart} Metering"

Section 1252 of EPAct 2005 requires that within 18 months of its enactment that states investigate and decide whether to mandate utilities to offer each customer a time-based rate schedule under which the rate charged by the electric utility varies during different time periods and reflects the variance, if any, in the utility's costs of generating and purchasing electricity at the wholesale level. The timebased rate schedule would enable the electric consumer to manage energy use and cost through advanced metering and communications technologies. If the states mandate time-based rate schedules, each electric utility would provide each customer requesting a time-based rate with a time-based meter capable of enabling the utility and customer to offer and receive such a rate, respectively. loggers/meters at the panel, circuit, or end-use level.

Operational-Opportunity Identification. The focus here is on building "tuning." This activity uses the metered data to validate that existing systems are operational and being controlled as expected/recommended. Significant savings have been documented (Gregerson 1997; Haasl and Sharp 1999; PECI 1997; Texas A\&M 2002, Mills 2009) in this area by identifying a variety of inefficiencies including inoperable night-time setback features, by-passed variable frequency control devices, and defeated energy-efficiency measures such as heating, ventilation, and air conditioning (HVAC) economizers and boiler/chiller controls. 
Power Quality Applications. Advanced meters can capture electrical anomalies such as transients, voltage disturbances, power factor, and harmonics in order to troubleshoot power quality problems. This can be especially useful when monitoring sensitive loads. Transients can cause premature failure of power-sensitive electronics in computers and other electronic equipment. An abnormally low power factor (usually a result of multiple and/or large inductive electric loads) can result in surcharges from utility companies. Using advanced meters will allow detection and documentation of power quality problems so solutions to those problems may be developed and implemented prior to equipment failure or high energy bills.

Measurement and Verification of ESPC Savings. In the Federal setting, energy savings performance contracts (ESPCs) often involve the installation of efficiency measures in a small number of buildings on a large campus-type facility that contains a single revenue meter. The savings generated by the ESPC can be a small fraction of the facility's total energy use, and can be difficult to estimate these savings from analysis of monthly utility bills. For this reason, indirect methods such as modeling and engineering calculation are often used to estimate savings. If the buildings are individually metered, readings before and after the project can be used to establish both the energy-use baseline and the energy savings to a much higher degree of confidence. In many cases, there is a need to move from whole-building to enduse (i.e., metering at the equipment) to capture accurate savings estimates.

Emergency Response. During an electrical power emergency (such as experienced in California in 2000 and 2001), or during other utility shortages (such as the water drought in 2002), the manager of a Federal facility may need real-time information in order to make decisions regarding physical plant closure or interruption of non-critical loads. A manager may want real-time feedback and, that the directions to staff to reduce utility use are being followed and are achieving the required result.

Benchmarking. Utility benchmarking is a process of collecting and trending building-level utility data for the purpose of comparison to the building's historic data or to a comparative building's data. Often, building benchmarking is accomplished using some normalization metric; for example: energy use per unit area ( $\mathrm{kWh}$ /square foot/year) or water use per occupant (gallons/occupant/year). The value of benchmarking lies in the ability to develop comparisons usually relevant to some accepted baseline condition. Once generated, these comparisons become the basis for further investigation for operational improvements.

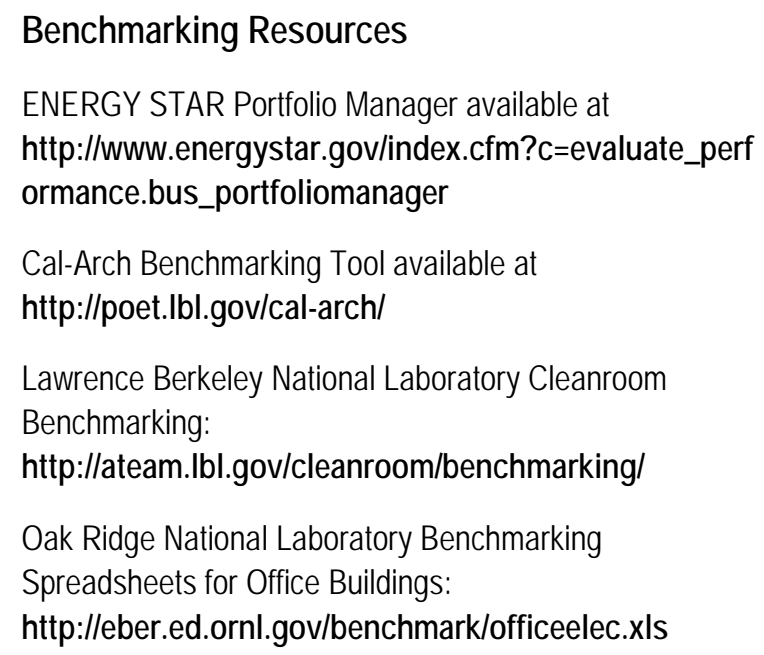




\subsection{Working with Data - Examples from the Field}

Interval data offer many benefits in identifying efficiency and operational opportunities. However, the data - even after processing - require active viewing to search for variability and trends.

Below is a collection of whole-building and end-use data for which some sort of variance was noted. These are presented to highlight the practical uses of interval data, what these data look like, and how evident some of the anomalies can be - once you start looking.

\subsubsection{Interval Data for Efficiency Opportunity Identification}

Objective: Use interval data to identify project opportunities.

Situation: Small administrative building at Federal site. Standard hours of operation, typical occupancy density, whole-building interval electric meter installed.

Findings: Viewing the daily demand profile, notice relative "flatness" of profile from day to night. Flat demand profiles are indicative of buildings with 24-hour occupancy, or a disabled nighttime setback control feature. In this case, the control had been disabled to accommodate a series of night meetings held in the building and never reset. Figures 7.1 and 7.2 below present the data as found and corrected.

Outcome: Once nighttime setback control feature is enabled (see Figure 7.2), significant decrease in nighttime electrical load - predominantly fan loads. Figure 2.2 indicates fan activation only in the early morning and late evening hours. The relatively high baseload may be candidate for lighting control retrofit or plug load analysis.

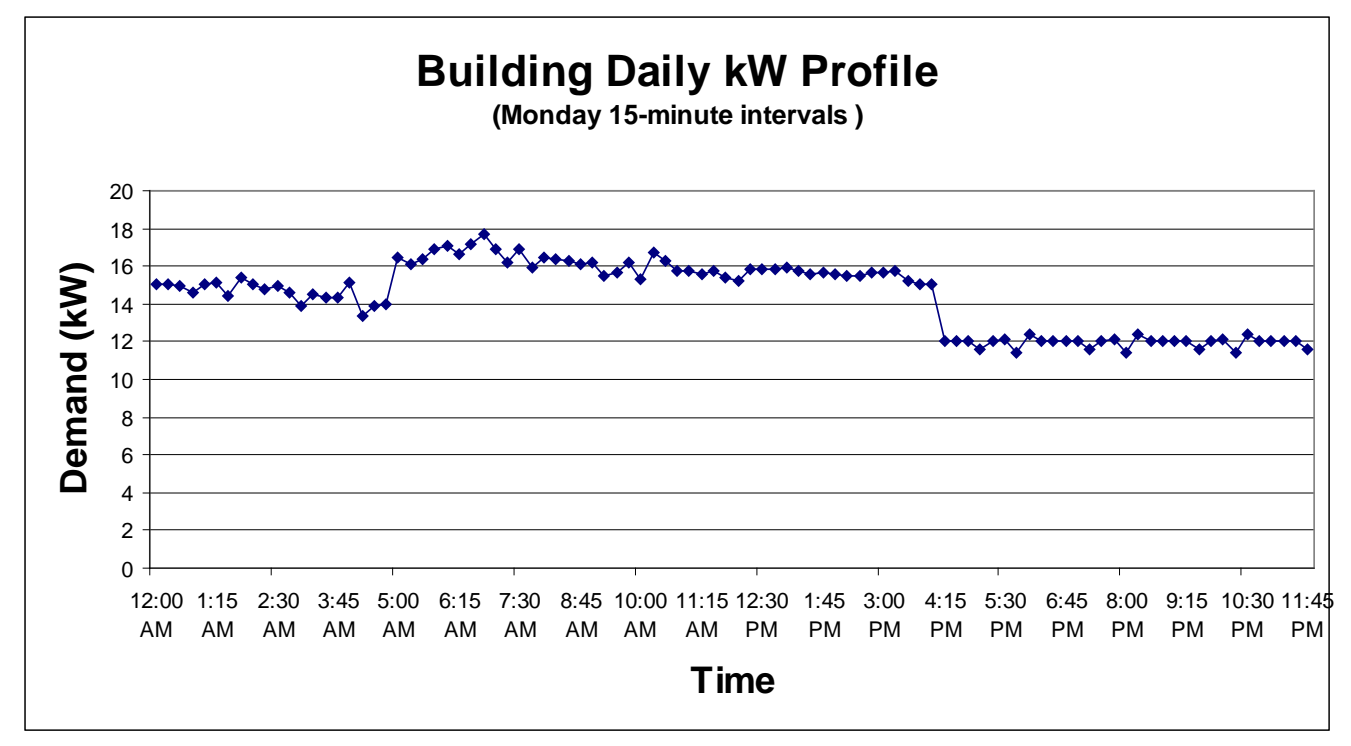

Figure 7.1. Daily Demand Profile - Nighttime Temperature Setback Disabled 


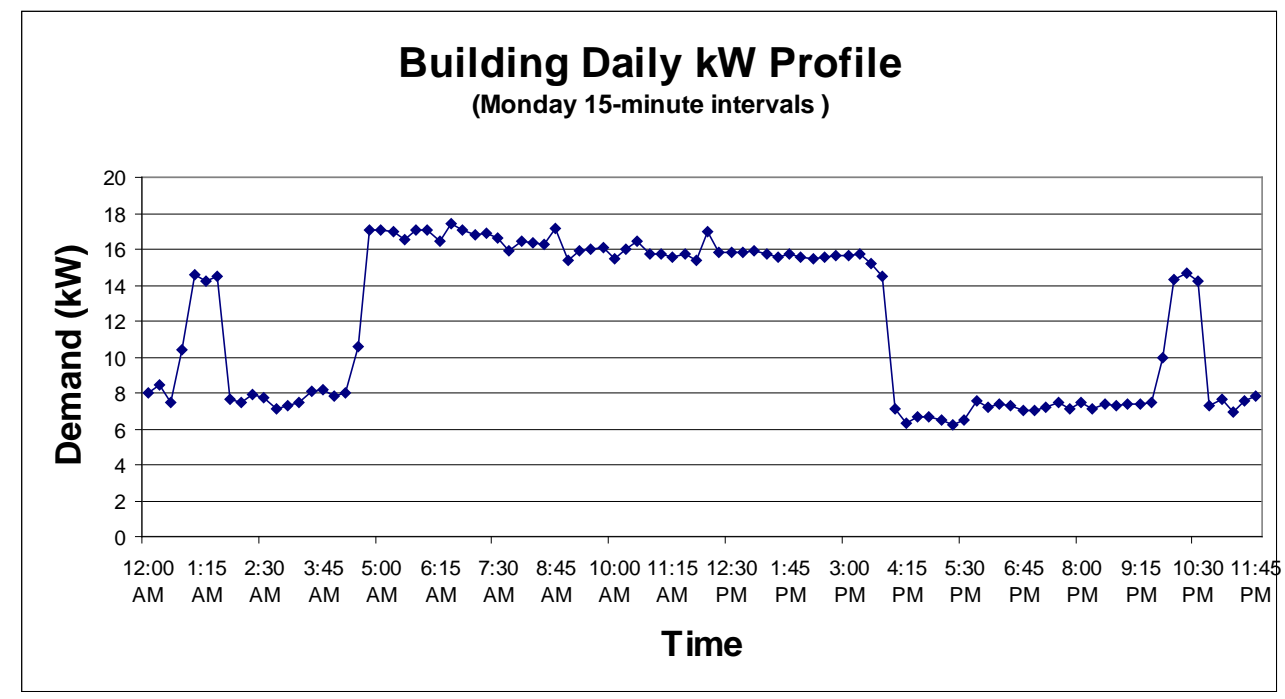

Figure 7.2. Daily Demand Profile - Nighttime Setback Enabled

\subsubsection{Building Benchmarking}

Objective: Use benchmarking to compare building energy performance $\mathrm{kWh} / \mathrm{ft}^{2} /$ month.

Situation: Two small, co-located training facilities with similar occupancy and hours of operation. Whole-building interval electric meter installed.

Findings: Viewing the monthly benchmark data (see Figure 7.3) shows Building A using roughly 20 percent more energy per square foot, each month.

Interval data offer
many benefits in
identifying efficiency
and operational
opportunities.
While there are a number of factors that could contribute to this, including occupancy variance, materials of construction, age/operation of equipment, and hours of operation - this type of variance is worthy of further exploration.

Outcome: Further exploration identified high nighttime lighting loads in Building A as part of the variance. Diverging load profile after December needs further exploration.

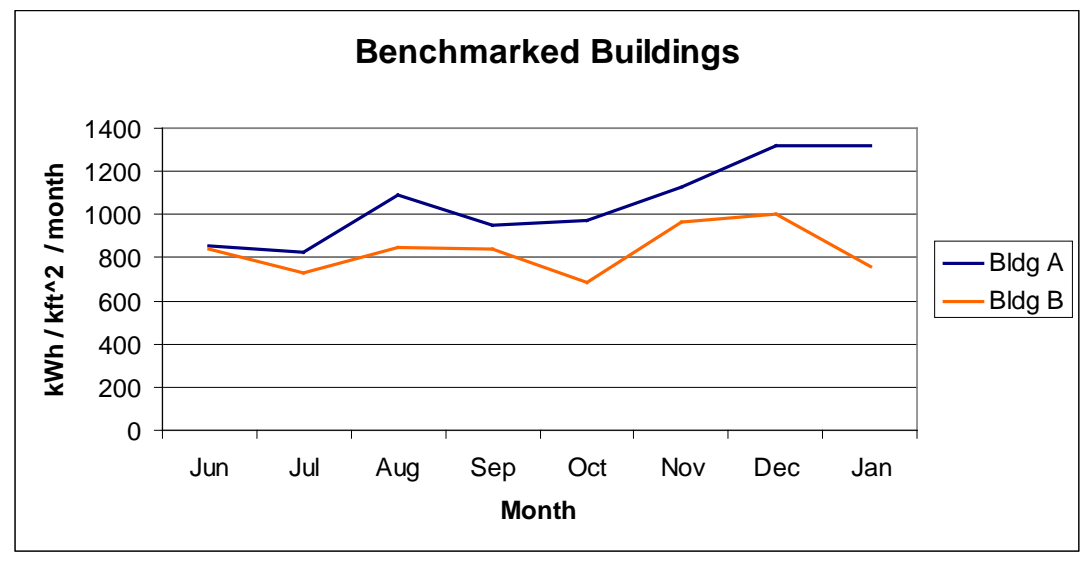

Figure 7.3. Building Benchmarking 


\subsubsection{Operational Efficiency Identification}

Objective: Use interval water-use data to identify water inefficiency.

Viewing the daily interval water-use data highlights suspicious use pattern, particularly during off-hours.
Situation: Trending of water use in Federal administrative building; wholebuilding interval water meter installed.

Findings: Viewing the daily interval water-use data highlights suspicious use pattern (see Figures 7.4 and 7.5), particularly during off-hours.

Outcome: Reviewing interval data for unoccupied period - see Figure 7.5 Saturday highlights suspicion of water leak. Leak confirmed as leaking restroom fixtures in men's bathroom.

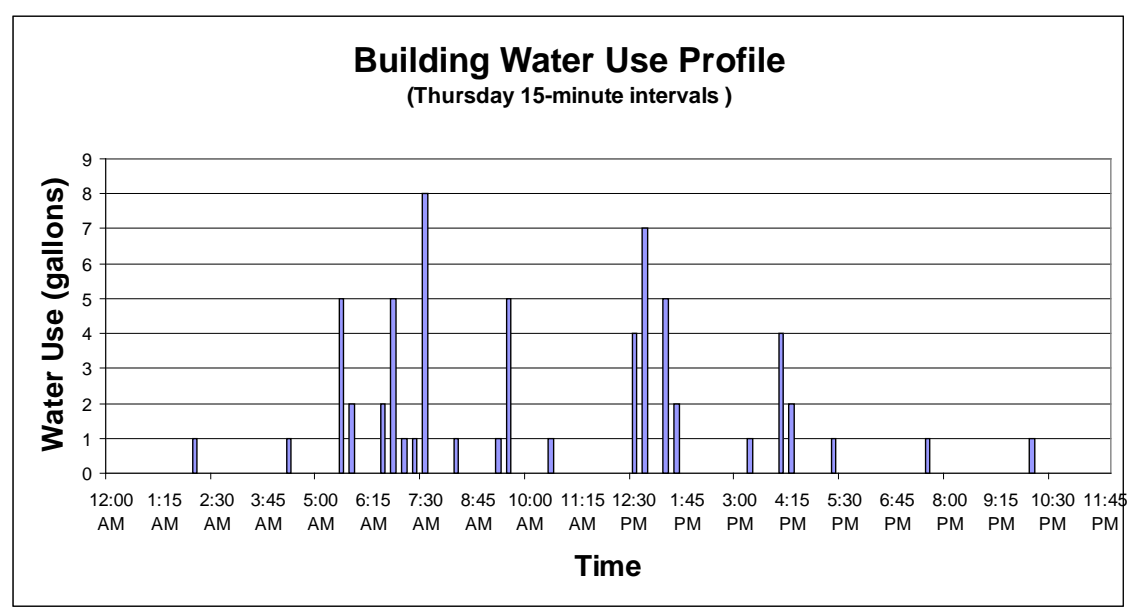

Figure 7.4. Building Water-Use Interval Data - Suspicious Pattern

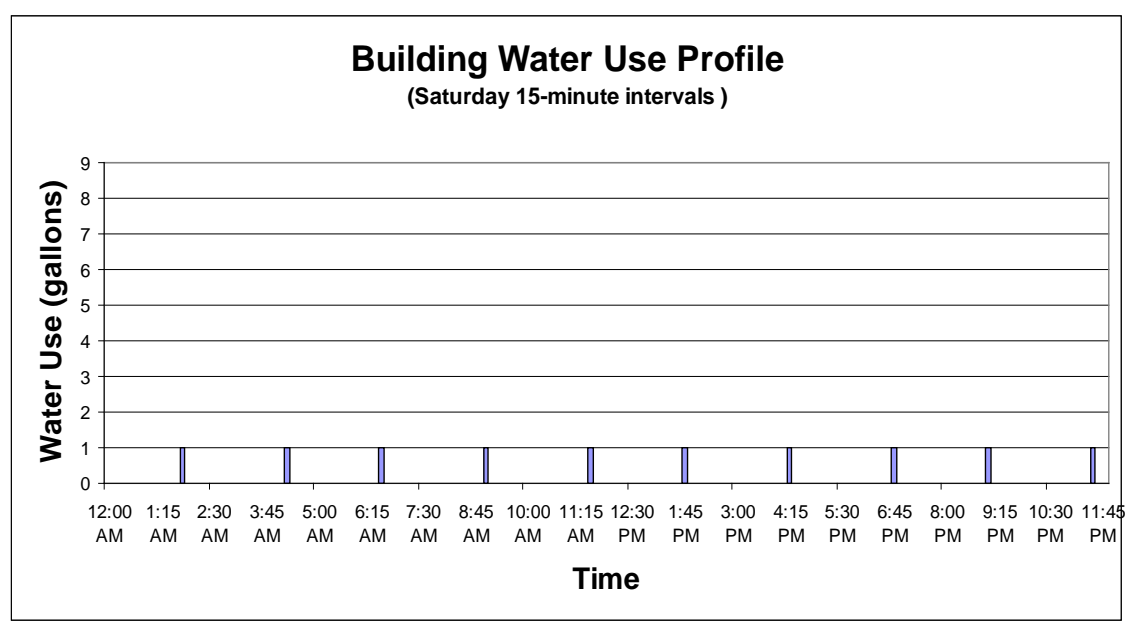

Figure 7.5. Building Water-Use Interval Data - Verification of Water Leak 


\subsubsection{Peak Demand Identification/Reduction}

Objective: Use interval demand data to identify, value, and ultimately reduce peak demand (LBNL 2004).

Situation: Federal facility with process loads and peaking demand. Wholebuilding interval electric meter installed.

Findings: By building a load duration curve - curve presenting the number of hours (or, as presented, percent of time) that a building's demand is greater than some predetermined value - opportunities for valuing and reducing peak

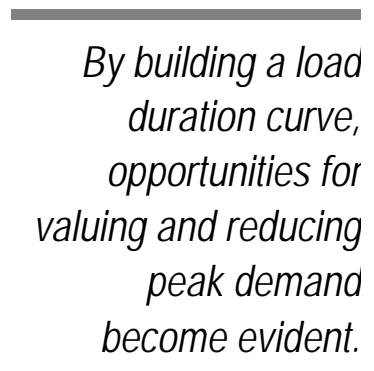
demand become evident. In the graph and accompanying data below (Figure 7.6 and Table 7.1), it is evident that for only 1 percent of the time (or about 7.5 hours) during the month of July the demand was above 2,102 kW.

Outcome: Given that peak demand for the month was 2,289 kW allows for calculation of the difference between the peak and the 1 percent value $(2,289-2,102)=187 \mathrm{~kW}$. This difference, $187 \mathrm{~kW}$, represents demand that occurred during just 7.5 hours in the month. At a peak demand charge of $\$ 17.92 / \mathrm{kW}$, this demand cost over \$3,350 for this month. Put differently, if this demand could have been avoided (during those 7.5 hours), the monthly savings of \$3,350 could have resulted. This general concept is scalable (i.e., consider avoiding the top 2 percent) and, when spread over 12 months, can have a significant monetary impact. To affect these savings, a clear understanding of the site's utility rate structure (not always an easy endeavor) is necessary.

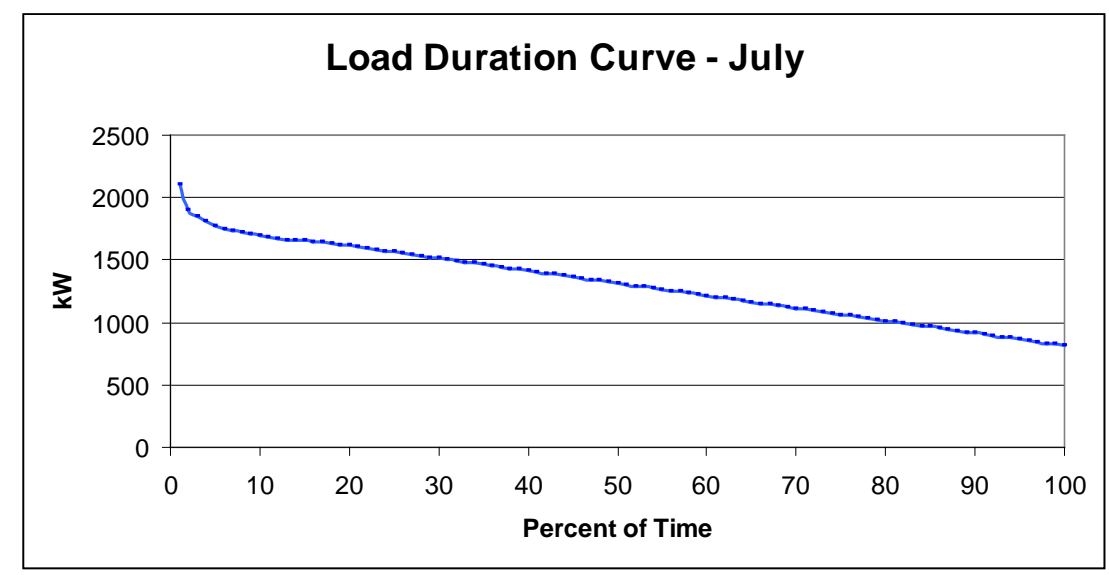

Figure 7.6. Load Duration Curve

Table 7.1. Load Duration Curve Data

\begin{tabular}{|c|c|}
\hline Percentage of Time (Month of July) & Electric Demand from Interval Data \\
\hline Peak & 2,289 \\
\hline $1 \%$ & 2,102 \\
\hline $2 \%$ & 1,905 \\
\hline $3 \%$ & 1,849 \\
\hline$\%$ & 1,807 \\
\hline $5 \%$ & 1,768 \\
\hline
\end{tabular}




\subsubsection{Operational Efficiency Opportunity Using Data Logger Data to Validate Boiler Operation}

Reviewing the

5-minute run-time

data reveals

excessive cycling of

boiler; Figure 7.7

presents these data.
Objective: Use data logger (5-minute run-time, time-series data) to validate proper boiler operation.

Situation: Federal facility with boiler heating/process loads. End-use run-time data logger installed. Data reported are from peak-season loading conditions.

Findings: Reviewing the 5-minute run-time data (data collected with standalone magnetic field enabled logger placed near boiler combustion-air blower motor) reveals excessive cycling of boiler; Figure 7.7 presents these data. A bar in the figure rising from 0 to 1 indicates the boiler cycling "on," the bar returning from 1 to 0 indicates the boiler cycling "off.” Therefore, each bar in the graph represents one on/off cycle.

Outcome: Processing these data reveals an average of 6.5 on/off cycles per hour - far in excess of the recommended 1-2, depending on load conditions. Further exploration uncovered gross boiler over-sizing due to partial decommissioning of building/process loads. The outcome recommendation includes installation of smaller, properly sized, and more efficient boiler to carry load.

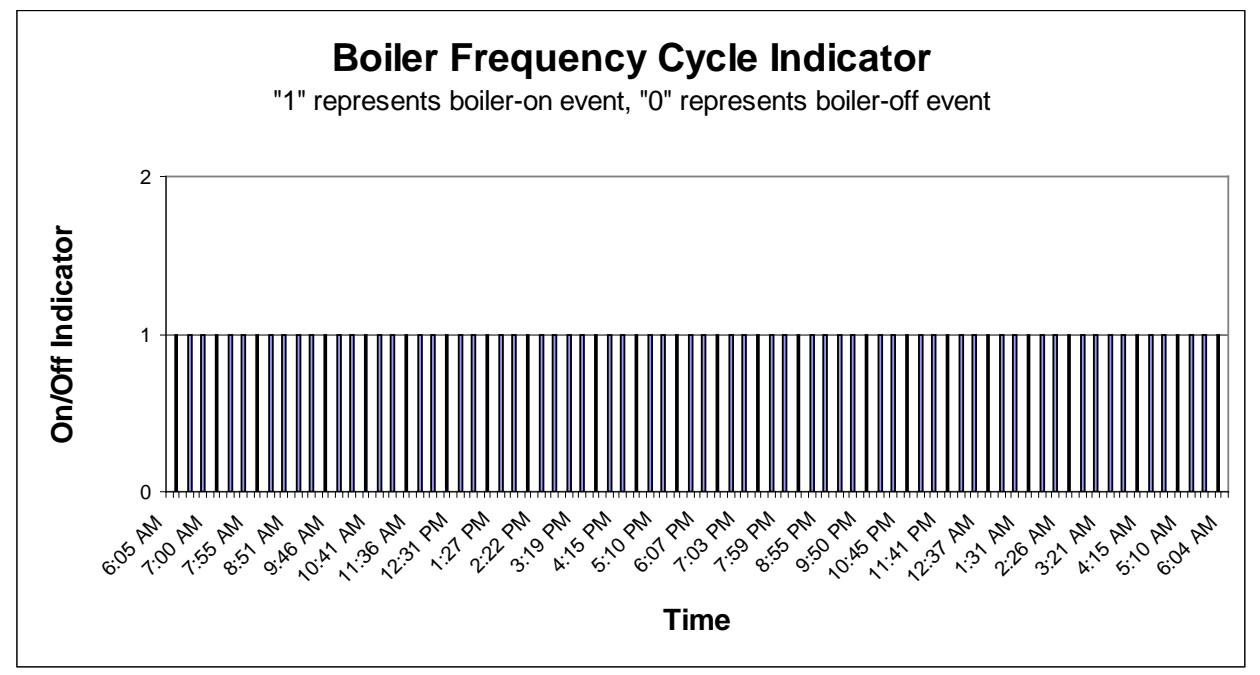

Figure 7.7. Boiler Cycling Frequency Data

Processing of the lighting panel data shows significant reduction in demand $(\mathrm{kW})$ of the retrofit technology.

\subsubsection{Measurement and Verification of New Lighting Technology}

Objective: Use electrical panel level (lighting circuit) demand (kW) data to verify manufacturer's claim of energy savings from new lighting technology (spectrally enhanced fluorescent lighting) installed at Federal facility.

Situation: Measurement and verification of retrofit lighting installed at large administrative space on Federal site. Panel-level interval metering installed. 
Findings: Processing of the lighting panel data shows significant reduction in demand $(\mathrm{kW})$ of the retrofit technology (spectrally enhanced fluorescent lighting) compared to the baseline technology (existing T-8 fluorescent lighting) with a clear presentation of savings. Figure 7.8 presents these data.

Outcome: Savings validate manufacturer's claim of performance.

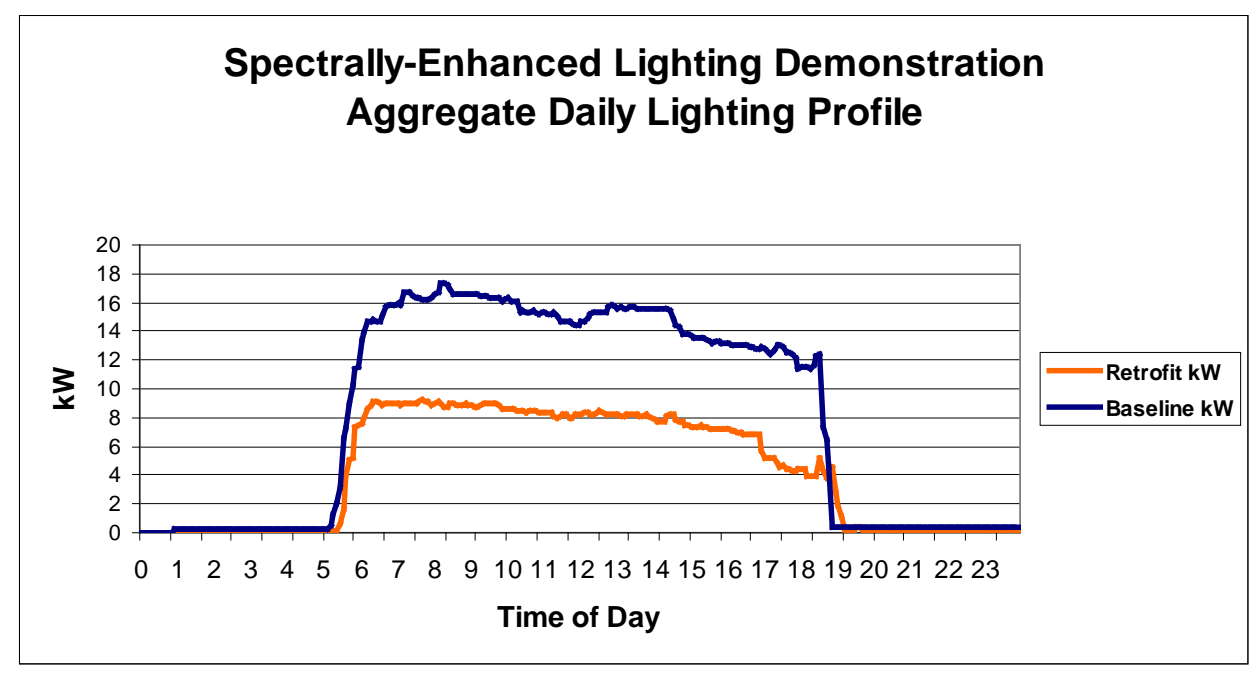

Figure 7.8. Measurement and Verification of Lighting Demonstration

\subsubsection{Data Center Monitoring}

Data center presence and capacity is rapidly growing in both the public and private sectors. This growth, fueled by the Internet economy and an ever increasing need for information storage and processing, has transformed data centers into one of the most energy-intensive building types and end-uses. As shown in Figure 7.9 (CEE 2009), annual data center energy cost is second only to that of the clean room and more than four times that of a hospital.

\section{Comparative Energy Costs High-Tech Facilities vs. Standard Buildings}

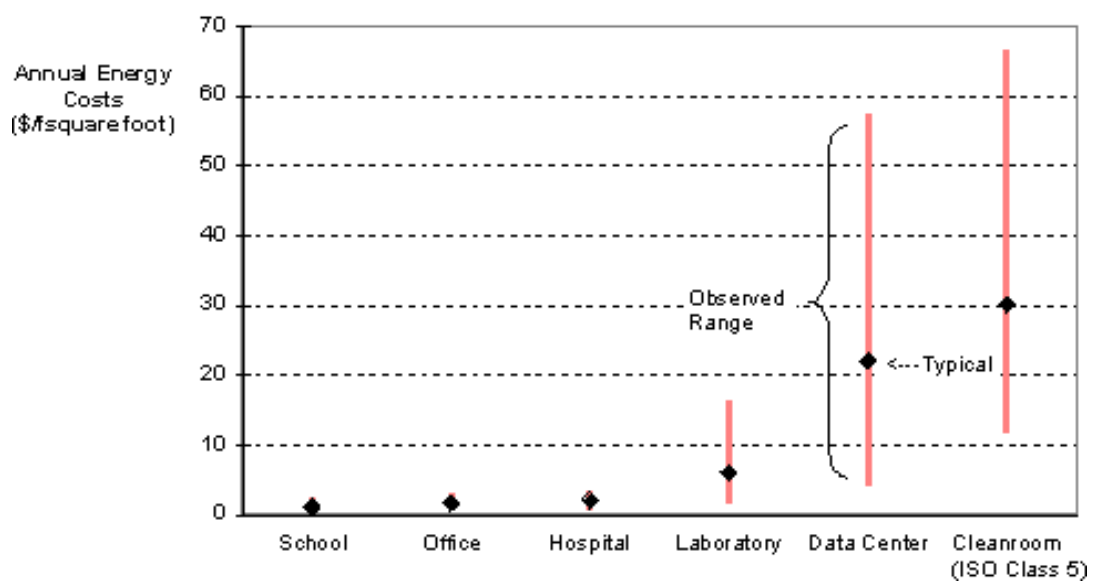

Figure 7.9. Comparative Annual Energy Costs of Different Facility Types 
Different from most other Federal sector metering applications, data centers require the need to both understand how energy is being used and how a variety of parameters may impact system reliability. It is not uncommon for a data center to require a reliability of "six nines"; put as a function of downtime, "six nines" is equivalent to 31.5 seconds per year of downtime. As such, the metering used to achieve this high reliability is typically more sophisticated, detailed, and expensive. Depending on a facility's function and data needs, a data center can be located in a small closet/communications area, a dedicated data center room, or occupy an entire floor. A typical data center room is shown in Figure 7.10 (CEE 2009).

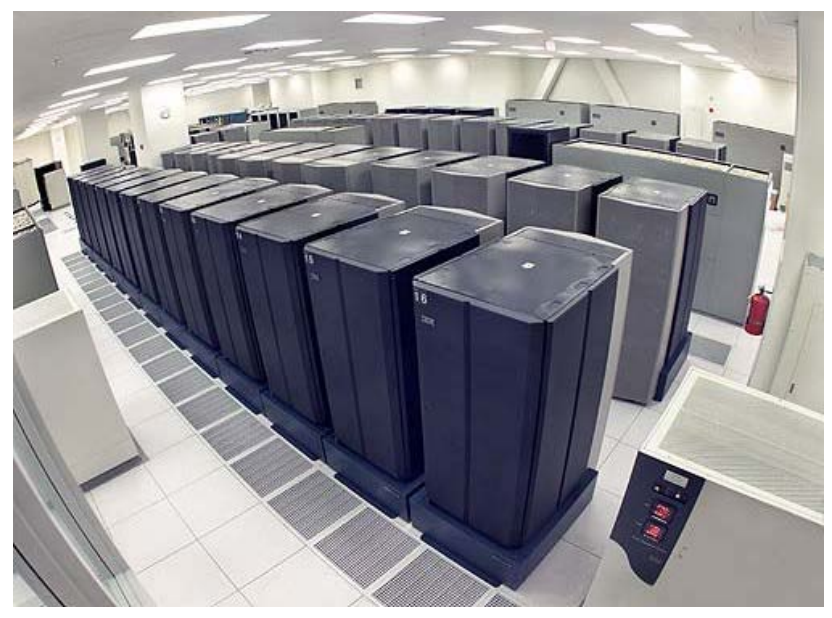

Figure 7.10. Typical Data Center

When a data center experiences an outage, it is usually due to power quality issues, circuit overloading, or human error. In each of these cases, proper metering will afford predictive diagnostics and thus reduce potential downtime. To achieve the highest levels of reliability, considerations for metering in data centers should include:

- Whole building electric

- Service panel mains and sub panels

- Critical HVAC/computer room air conditioning (CRAC) systems

- Back-up generators

- Uninterruptible power supplies (UPSs)

- Critical Data communications equipment

- Server rack breakers.

The actual points and parameters to collect will vary with data center operation and equipment. The key diagnostic for each of these parameters is a noted "change in value"; specific diagnostics will be a function of system type/operation and beyond the scope of this document. However, through trending of these data points, "exception reports” and "out of range” values will be apparent and warrant further investigation. 
Typical metering will include the full suite of diagnostics for the above points and include:

- Voltage regulation and imbalance. Voltage imbalance relates to the maximum deviation from the average of the three-phase voltages or currents. A typical source of voltage imbalance is single-phase loads on a three-phase circuit or the dropping of one phase of the circuit due to failure of circuitry, fusing, or poor connections. Diagnostics include monitoring of:

- Phase voltages, both individual and phase-to-phase

- $\quad$ Root mean square (RMS) magnitudes

- Sampling of maximum, minimum, and averages

- Voltage sags and swells. These two parameters are defined as decreases (sags, also known as dips) or increases (swells) in system voltage. The predominant causes of voltage sags and swells relate to the switching on (sags) and off (swells) of large loads (e.g., process or HVAC motors) that have large start-up current draws. Diagnostics include monitoring of:

- Phase voltages and currents

- Equipment response

- Power factor. The ratio of true power to volt-amperes in an AC circuit. Power factor (PF) is expressed in a percent of unity (lagging for inductive loads or leading for capacitive loads; resistive loads have a unity $\mathrm{PF}$ ) and have economic implications depending on the utility rate structure.

Diagnostics include the monitoring of:

- PF measured at the service feed, breaker, or end-use level

- $\quad$ Correction considered for values $<0.85$

- Harmonics/harmonic distortion. Harmonics represent the sinusoidal voltages (or currents) with frequencies that are integer multiples of the operational system frequency. Harmonic distortion relates to the nonlinear characteristics of the loads on the system and the impacts these may have on sensitive loads. Diagnostics include monitoring of:

- Phase voltages and currents

- Waveform characteristics

- Neutral conductor loading

- Trend analysis to predict impending issues

Because of the critical nature of this metering, it is suggested that a detailed metering plan specific to the application be developed. Plans of this nature can be very complex and are best developed with IT staff knowledgeable of both the system and metering equipment capabilities.

\subsubsection{Data Center Benchmarking}

Beyond the reliability needs for data centers, energy efficiency is becoming a very closely monitored attribute. A key metric for data center energy use is the power usage effectiveness (PUE) and is defined as:

\section{Power Usage Effectiveness $($ PUE) = Total Facility Power/Total IT Equipment Power}


where

Total Facility Power = all power delivery components (external to the IT equipment), all HVAC, lighting, and other miscellaneous loads.

Total IT Equipment Power = all loads directly associated with IT equipment including storage hardware and computers used to monitor or control data systems.

Benchmarks for PUE have been developed and are constantly being assessed for validity. Current benchmark PUE values are presented in Table 7.2 (FEMP 2010a):

Table 7.2. Benchmark Data Center Power Usage Effectiveness

\begin{tabular}{|c|c|}
\hline Benchmark Level & Power Usage Effectiveness (PUE) \\
\hline Standard & 2.0 \\
\hline Good & 1.4 \\
\hline Better & 1.1 \\
\hline
\end{tabular}

\subsubsection{Available Software Tool}

\section{Data Center Energy Profiler (DC Pro)}

Description: The Data Center Energy Profiler, or DC Pro, is a free online software tool provided by the U.S. Department of Energy to help industries worldwide identify how energy is being purchased and consumed by their data center(s) and also identify potential energy and cost savings. DC Pro is designed so that the user can complete a data center profile in about an hour. When a DC Pro case in completed, a customized, printable report is developed that shows the details of energy purchases for the data center, how energy is consumed by the data center, potential cost and energy savings, comparison of the data center energy utilization versus other data centers, and a list of next steps to start saving energy.

Availability: Version 1.1 of DC Pro released November 8, 2010, is available at http://dcpro.ppc.com/.

\subsubsection{Overcoming Common Metering Challenges}

In the course of any metering exercise there will be challenges that arise - these may occur in planning, specifying, purchasing, installing, or in the maintaining of the metering system. This section focuses on some of the more common metering challenges with the hope that identifying these early in the process mitigates any potential impact.

Metering Planning. The planning step of the metering process is critical to program success. Chapter 3 focuses on the key steps in this process. Some of the challenges in the planning process include:

- Best Information. Good information drives good planning and decision making. It is incumbent on the planners to make sure the best available information is accessed and used. In metering of existing buildings, access to electrical plans, one-line diagrams and panel schedules is critical. However, as is often the case, these documents (when available) are incomplete, outdated or are not considered "as-built." A recommended step in the planning process is the access and verification (possibly confirming with field inspection and spot measurement) of all technical documents. 
- Under/Overestimating Breadth of Project. A key to metering planning resides with an ability to estimate current and future needs. While it is difficult to accurately predict future needs, efforts to plan for future system expansion are critical to the economic success of the system. This is often done by scenario phase-planning whereby different project implementation assumptions are used to create best, most likely, and worst-case scenarios. These different scenarios are then accommodated for in the planning process.

- Management Buy-in and Support. Obtaining and maintaining management support for project planning and implementation is an often overlooked activity (FEMP 2010b). Providing management with regular updates on activities and successes is one way to keep the project visible and maintaining this support.

- Milestone Development. While many plans focus on the "what," effective plans also focus on the "how." Developing how and when the steps of the process will be implemented are critical to assuring a successful metering program.

Equipment Specification. The identification of the correct equipment is crucial to the project's success and longevity. This requires an ability to distinguish the necessary parameters for proper function.

- Accuracy. As defined, the accuracy of a metering system is the difference between the measured value and the actual value. While overall system accuracy is important and often reported for standard operating ranges, accuracy should be addressed for each component of the system and, importantly, over the entire range of expected values. Current transformers (CTs) and fluid meters present good examples of this need.

- CTs are usually accurate over all but the low end of their range. With normal CTs, the accuracy falls off significantly at low currents (e.g., those at less than 10 percent of the full-scale rating). CT sizing should be done with care and full knowledge of all expected measurement ranges, paying particular attention to the low end of the range.

- Fluid meters are often specified on average and maximum flow rates. In systems with a wide range, and including low flows, accuracy can suffer. In these cases, compound meters are often specified to accommodate the two different flow rate regimes to achieve desired accuracy.

- Operation. Prior to the identification and procurement it is important to understand the equipment; that is, its function, operation, and maintenance needs. This step must involve input from those who will be using the equipment. Parameters to consider include:

- Meter installation and set-up, paying particular attention to spatial constraints and code requirements

- Software configuration and ease of use

- Process and schedules for maintenance of system, sensors, and battery needs.

- Communications. Meters from different vendors or product lines can have different data sampling rates, sampling intervals, and communicate and transmit data using different protocols and formats (ACEEE 2010). To mitigate any potential issues with communication consider the following guidance:

- Standardization on manufacturer and/or data protocols. There are a number of established and developing protocols (e.g., BACnet, LonWorks, Modbus, pulse); make certain that the meters specified have a common protocol or can be converted for proper communication. 
- Data collection intervals. To assure accurate data processing, meters should be set to a universal time stamp (i.e., time series records reference the same time stamp) and be collecting and integrating over the same interval (e.g., a 15-minute interval).

- Data interoperability. Depending on how data are collected and processed, routines may need to be employed that pre-condition or consolidate the data for final processing.

- Cost of Ownership. An important metric in specification is total cost of ownership over the equipment's life. Identifying what the defined life of the system is the first step. In addition, it is important to determine the recurring cost, including any periodic calibration, needs for part/sensor replacement, or upgrades to system software or licensing. All of these parameters should be readily available from equipment vendors and should be received in writing.

Purchasing. The details and specifications of items procured are very important when purchasing metering equipment and accompanying sensors. Procurement specifications should be developed with engineering and facilities management input/oversight and include a thorough review before any purchase orders are signed.

- Manufacturer Standardization. While it is not always possible, there are some advantages to standardizing on one equipment manufacturer; these include:

- Single source of information and product

- Minimization of finger pointing when "system" problems occur

- Volume procurement/discount opportunities.

Installation. Following all manufacturers' guidelines and relevant code requirements is essential to proper and safe installation. Additional recommendations to consider include the following:

- Offer an explicit instruction set for all installations, provide relevant contacts for installation questions, and develop checklists for installation check out. Typical installation faults include:

- CT directionality

- Voltage and phase consistency

- Meter constants, inputs, and programming.

- Develop installation commissioning protocols. Verify each meter individually.

- Develop "expected" values for each meter.

- Compare output against "expected” values and spot measurements.

- In multi-meter installations verify that the sum of the parts equals the whole

- Individual end use, sub-panel and whole building.

- Develop communication commissioning protocols.

- Verify each meter individually.

- Confirm time series output received at endpoint/data center.

- Confirm summed data received over duration (week/month) are accurate and expected. 
Maintenance. Once installed and operating, facility staff must focus on keeping metering systems productively functional. Meter/sensor calibration, data receipt, and accuracy are the key meter maintenance parameters most often neglected. To prevent these issues from arising, the following are suggested:

- Consider developing time-based checklists of manufacturer's maintenance recommendations and including these in regular maintenance activities (FEMP 2010b).

- If the site uses a computerized maintenance management system (CMMS), all relevant metering information (manufacturer, model, date of installation, size, and procedures) should be entered into the CMMS from where automated work orders will be generated.

\subsection{References}

American Council for an Energy-Efficient Economy (ACEEE). 2010. Toward the Holy Grail of Perfect Information: Lessons Learned Implementing an Energy Information System in Commercial Buildings. K Kirchner, G Ghatikar, S Greenberg, D Watson, R Diamond, D Sartor, C Federspeil, A McEachern, and T Owen, ACEEE Summer Study on Energy Efficiency in Buildings, Washington, D.C.

California Energy Commission (CEC). 2007. Enhanced Automation Vendor/Contractor List. Sacramento, California. Available at: http://www.energy.ca.gov/enhancedautomation/documents/EA_Vendor_List.pdf.

California Energy Commission (CEC). 2009. Save Energy Now in Federal Data Centers: An LBNL Case Study. Presentation to the Consortium for Energy Efficiency (CEE) June 2009 Meeting, Boston, Massachusetts.

Canadian Industry Program for Energy Conservation (CIPEC). 2004. Energy Management Information Systems. Natural Resources Canada, published by the Office of Energy Efficiency of Natural Resources Canada.

Energy Policy Act of 2005 (EPAct 2005). 2005. Public Law 109-58, as amended, Section 103, Energy Use Measurement and Accountability, Section 543 (42 USC 8253), (e) Metering of Energy Use.

Federal Energy Management Program (FEMP). 2010a. Best Practices Guide for Energy-Efficiency Data Center Design. Developed for the U.S. Department of Energy, Office of Energy Efficiency and Renewable Energy by the National Renewable Energy Laboratory, Golden, Colorado.

Federal Energy Management Program (FEMP). 2010b. Operations \& Maintenance Best Practices: A Guide to Achieving Operational Efficiency, Release 3.0, prepared by Pacific Northwest National Laboratory for the Federal Energy Management Program, U.S. Department of Energy, Washington, D.C. Available at: http://www1.eere.energy.gov/femp/pdfs/omguide_complete.pdf.

Granderson J, MA Piette, G Ghatikar, and P Price. 2009. Building Information Systems: State of Technology and User Case Studies. LBNL-2899E, Lawrence Berkeley National Laboratory, Berkeley, California.

Gregerson J. 1997. Commissioning Existing Buildings. TU-97-3, E Source, Boulder, Colorado. 
Haasl T and T Sharp. 1999. A Practical Guide for Commissioning Existing Buildings. ORNL/TM-1999/34, Oak Ridge, Tennessee.

Lawrence Berkeley National Laboratory (LBNL). 2006. Enterprise Energy Management System Installation: Case Study at a Food Processing Plant. LBNL_STAC, Berkeley, California.

Lawrence Berkeley National Laboratory (LBNL). 2004. Using Energy Information Systems (EIS): A Guidebook for the U.S. Postal Service. LBNL-54862, Berkeley, California.

Lawrence Berkeley National Laboratory (LBNL). 2002. Web-Based Energy Information Systems for Large Commercial Buildings. LBNL-49977, Berkeley, California.

Mills E. 2009. Building Commissioning: A Golden Opportunity for Reducing Energy Costs and Greenhouse Gas Emissions. Report prepared for the California Energy Commission, Public Interest Energy Research. Lawrence Berkeley National Laboratory, Berkeley, California. Available at: http://cx.lbl.gov/documents/2009-assessment/lbnl-cx-cost-benefit.pdf.

Portland Energy Conservation, Inc. (PECI). 1997. What Can Commissioning Do For Your Building? Federal Energy Management Program, U.S. Department of Energy, Washington, D.C.

Texas A\&M. 2002. Continuous Commissioning Guidebook for Federal Energy Managers. Federal Energy Management Program, U.S. Department of Energy, Washington, D.C. 


\section{Chapter 8 Federal Sector Case Studies/Success Stories}

\subsection{Electric Metering Program at Brookhaven National Laboratory}

$\underset{\text { NATIONAL LABORATORY }}{\text { BROOKHEN }}$

Brookhaven National Laboratory (BNL), centrally located on Long Island, New York, is a multi-program national laboratory operated by Brookhaven Science Associates for the U.S. Department of Energy (DOE), and employs over 2,700 people. Major research programs resident at the laboratory include

- Nuclear and high-energy physics

- Physics and chemistry of materials

- Environmental and energy research

- Nonproliferation

- Neurosciences and medical imaging

- Structural biology.

Brookhaven National

Laboratory's

metering program

began in the mid-

1980s in response to

the installation of

Electricity is purchased on the real-time market by the hour using the New York Power Authority (NYPA) as its purchasing agent and is transmitted to the laboratory by the Long Island Power Authority (LIPA). The annual electric bill for fiscal year 2006 was $\$ 16$ million, with consumption exceeding $241 \mathrm{GWh}$. new buildings and energy-intensive process loads. The average blended cost of electricity to the site is 6.5 cents per kWh. However, hourly prices for electricity can exceed $\$ 1 / \mathrm{kWh}$ during certain periods. Further, the impact from demand charges is over $\$ 100,000 / \mathrm{MW}$-year.

The BNL metering program began in the mid-1980s in response to the installation of new buildings and energy-intensive process loads. The addition of new buildings and additional process loads (e.g., supercomputer installations) continues today, all contributing to the already large site electricity bill.

The initial objective of BNL's metering program was to better control electric demand and energy use through billing the resident programs for their actual electric use (cost allocation). Approval of the metering program was based on the idea of controlling electricity costs due to increasing energy use by identifying areas of increasing consumption and validating cost savings from energy conservation measures.

Two main metering projects at BNL were funded by DOE in the 1980s. Since that time, all metering expenses have been covered by site operating funds.

BNL currently has over 200 electric meters onsite metering building loads, substations, individual transformers and process loads. Pulse data from the electric meters are stored in digital data recorders. The data recorders are interrogated on a daily basis by a meter data retrieval program via an analog phone line connection. The data are stored on a server, which is then used for monthly bill calculations, load analysis, and database input. 
Figure 8.1 is used by BNL to present a monthly summary comparison of the actual site electric load to the planned site electric load. Electric load planning and management are critical activities at BNL as the site purchases all its electricity in the day-ahead market. Deviations from the scheduled usage are either purchased or sold in the real-time market where prices can fluctuate dramatically. In this case, the scheduled load is an estimate of the daily loads by hour. The consumption data are also presented on an hourly basis. Consumption and cost data are reviewed on a daily basis, and this information is made available to site tenants via a website.

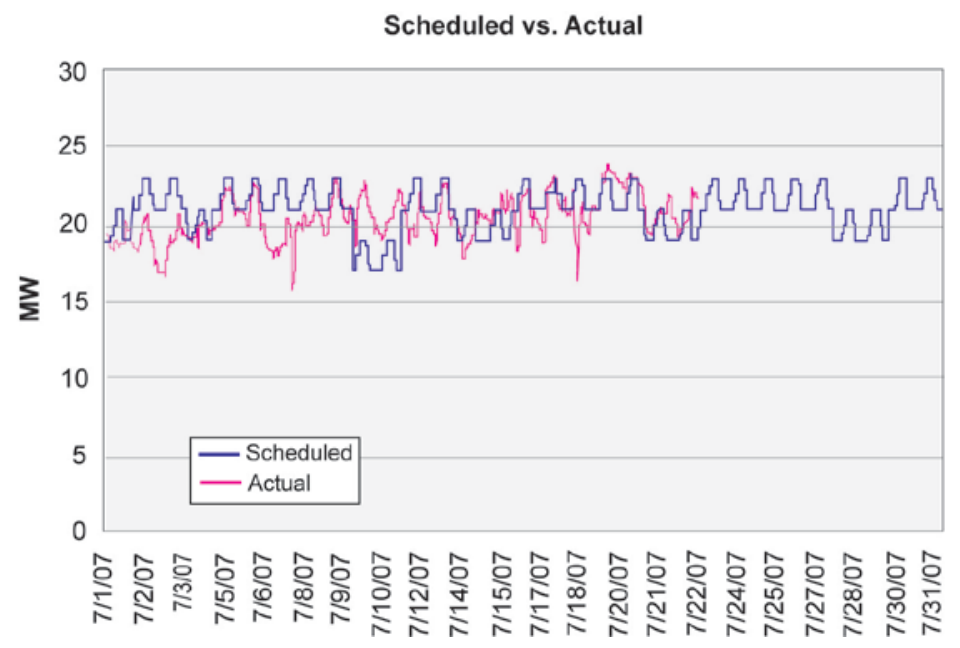

Figure 8.1. Monthly Summary of Scheduled vs. Actual Site Electric Demand Loads

The ability to monitor demand loads saves

BNL "well over

$\$ 1$ million per year."
Benefits in the form of cost savings resulting from the BNL metering program have not been documented over the course of the program's life. However, site staff offer the following observations:

- Electric consumption of one tenant dropped 40 percent when the tenant was billed for actual consumption. BNL has noticed similar behaviors when billing tenants for actual chilled water and potable water consumption.

- The ability to monitor and control demand loads saves the site "well over \$1 million per year.”

\section{Lessons Learned:}

- The metering system at BNL has allowed the site the flexibility to purchase electricity at the most favorable time-based rates, leading to significant savings.

- Load management in support of electricity procurement can be done at a large, multi-building site.

- Allocating costs to tenants on the basis of actual use can lead to significant modifications in behavior and use.

- Data captured by the electric meters are also used by electrical design engineers to properly size replacement transformers, generators, and conductors. 


\subsection{General Services Administration's Kastenmeier Federal Courthouse}

In 2005, the operations staff at the Kastenmeier Federal Courthouse in Madison, Wisconsin, agreed to serve as a pilot site for the demonstration of the newly developed web-enabled Whole-Building Energy Diagnostician (WBE). The WBE was originally developed by the Pacific Northwest National Laboratory (PNNL) with funding from the U.S. Department of Energy's (DOE's) Building Technologies Program. In an effort to make the tool more affordable and more widely available to the Federal sector, DOE's Federal Energy Management Program (FEMP) funded the development of a web-enabled version of the WBE. The WBE module installed at the Kastenmeier Federal Courthouse was a commercialized version of the tool.

The WBE module tracks energy uses at the building level - in this case, the total electric and natural gas use. The values of expected energy consumption are generated by empirical models of the building, which are automatically developed by the WBE. In general, the model uses time of week, outdoor air dry-bulb temperature, and relative humidity as independent variables. The WBE then graphically provides building operators alarms for unexpected usage to identify major changes in energy consumption (PNNL 2005).

As part of the demonstration project, NorthWrite, Inc. partnered with FEMP to make the web-enabled WBE module available to the Madison Courthouse as part of an overall suite of operations and management tools. The Madison Courthouse is a 100,000-square-foot building in Madison, Wisconsin. The building spaces include court rooms, chambers for the judges, jury rooms, holding cells, and offices for the Clerk of Courts, Bankruptcy Courts, and

The Whole-Building Energy Diagnostician tracks energy uses at the building level in this case, the total electric and natural gas use in General Services Administration's Kastenmeier Federal

Courthouse. U.S. marshals. Daily building occupancy includes approximately 120 full-time employees plus daily visitors.

The one-year demonstration started in May 2005 with the installation of the electric pulse meter and a monitor device, which reads pulse outputs from the meter and sends them wirelessly to a network operations center, needed to support the web-enabled WBE (Figure 8.2). A gas pulse meter was installed in July 2005. Summary of costs to purchase, install, and operate the metering system at the Kastenmeier Federal Courthouse is as follows:

- \$1,000 to purchase and install the electric and gas meters (approximately \$500 each)

- \$3,500 to install the proprietary WBE-based tool hardware

- $\$ 2,500$ to train agency staff on the use of the commercial web-based suite of tools (which included the WBE-based tool as well as additional site maintenance management functionality)

- \$100 per month for the monitor service

- \$250 per month to subscribe to the WBE-based tool and commercial maintenance management services suite of tools. 


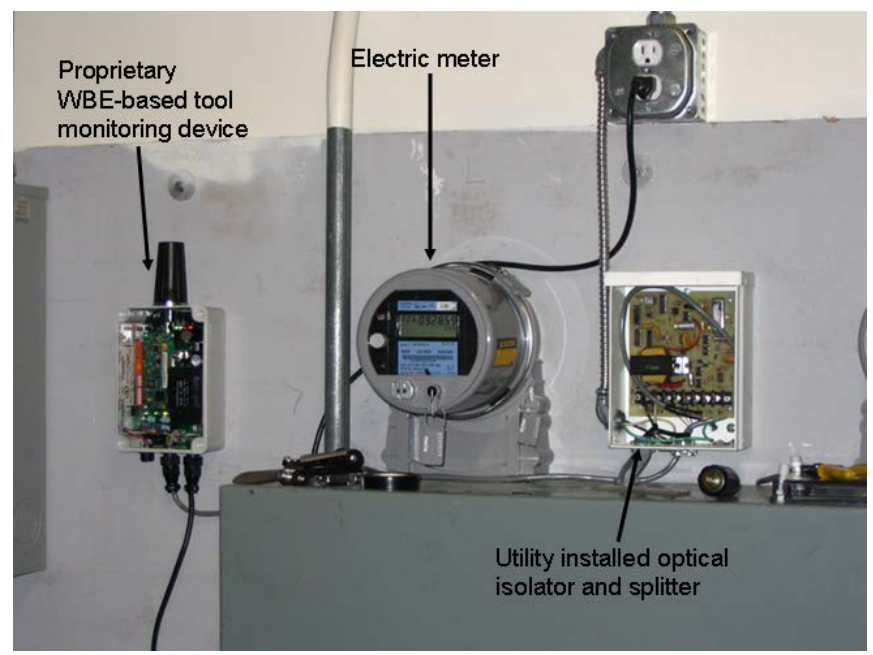

Figure 8.2. Installed Wireless Monitoring System for WBE-Based System (Photo courtesy of NorthWrite, Inc., Minneapolis, Minnesota.)

The reports and
graphics generated
by the whole-building
energy module are
reviewed daily by the
building mechanic as
part of the morning
startup.

The reports and graphics generated energy module are reviewed daily by the building mechanic as startup.
The reports and graphics generated by the WBE module are reviewed daily by the building mechanic as part of the morning startup, with an emphasis on verifying that peak usages do not vary unexpectedly (Figures 8.3 and 8.4). These daily reviews of the data have been helpful in diagnosing:

- Incomplete reprogramming of schedules on the building automation system (BAS) following a time change

- Improper boiler sequencing operations

- An air-handler operating 24/7 instead of on the schedule as programmed by the BAS

- Belt slippage due to wear on a large horsepower motor

- Refrigerant leaks in rooftop compressors.

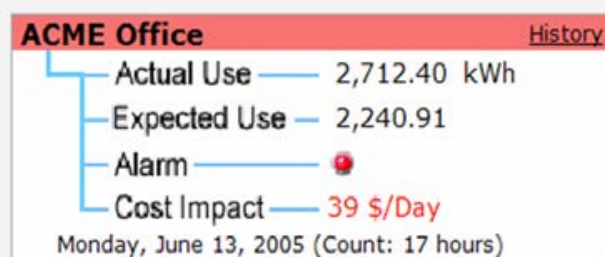

Figure 8.3. Sample Screen Capture for a Generic Building Showing an Alarm or High-Energy Using Condition. Included is an estimated cost impact associated with the higher than expected electricity use. (Screenshot courtesy of NorthWrite, Inc., Minneapolis, Minnesota.) 


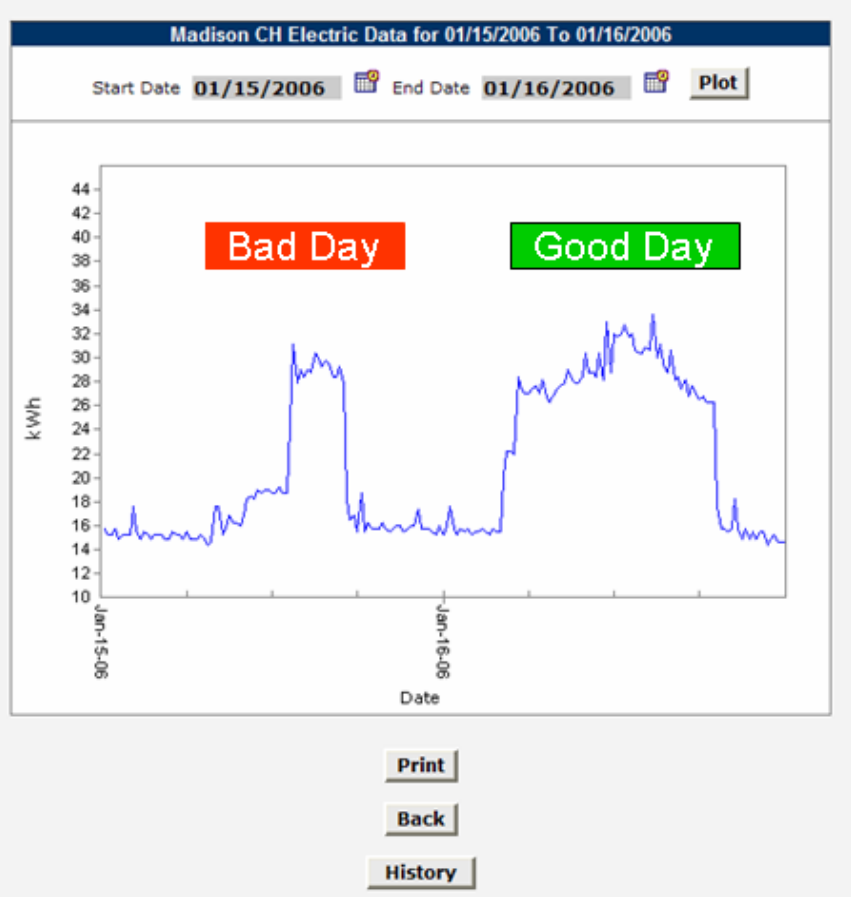

Figure 8.4. Data from the Monitoring Device Provides a Comparison of 2 Days of Electricity Use for Sunday, January 15, 2006, and Monday, January 16, 2006. Figure shows unexpected offhour usage on the 15th, while electricity consumption on the 16th was as expected. (Screenshot courtesy of NorthWrite, Inc., Minneapolis, Minnesota.)

The WBE module also lets the General Services Administration (GSA) staff

- Observe the effects of variable-speed drives and direct-expansion cooling operations, including occasional spikes in electrical consumption

- Verify that energy-intensive IT downloads are completed during off-hours.

Since this is a web-based system, the GSA regional energy office in Chicago is able to access and review the system data. This second set of eyes works to alert the Madison staff of other possible emerging trends.

Lessons Learned:

The whole-building energy diagnostician has been helpful in identifying problems such as improper boiler sequencing operations and refrigerant leaks in

- Metered data were instrumental in identifying high or abnormal energy use, rooftop compressors. and assisted in diagnosing inefficient equipment and systems operations at the Kastenmeier Federal Courthouse.

- The ability to view data at multiple, including remote, locations allowed for expanded assessment capabilities.

- The application services provider model allowed for expanded functionality through the offering of additional facilities management tools. 


\subsection{General Services Administration's Enterprise- Based Metering System (Curran 2007)}

The General Services Administration's (GSA's) Public Buildings Service is the largest real estate organization in the country. The GSA building inventory includes 179.7 million square feet of building space located nationwide that is tracked for compliance with the Federal energy reduction goals. This goal inventory accounts for over $\$ 300$ million in annual

GSA's building inventory includes 179.7 million square feet of building space located nationwide that is tracked for compliance with the Federal energy reduction goals. energy expenditures and includes over 880 facilities ranging from 817 square feet to 4.2 million square feet.

Efforts to establish and deploy an agency-wide metering system began in response to a 2004 Government Accounting Office (GAO) audit on demand response programs. ${ }^{11}$ In this report, the GAO found that participation in demand response programs by the GSA at a limited number of buildings had resulted in significant savings, and concluded that agency-wide participation in such programs would have resulted in significantly greater savings. The GAO audit, along with the soon-to-follow EPAct 2005 metering requirements, provided the impetus for GSA to develop and deploy an agency-wide metering system that could address a variety of energy management - use, procurement, and reporting - issues:

- Provide real-time interval data for energy use diagnostics

- Trend building electricity use on daily, monthly, and yearly basis

- Establish and manage peak demand usage where ratchet charges are in effect

- Promote participation in demand response program offerings

- Assist in aggregating load profiles for competitive power procurements

- Aggregate electricity use data on a national basis

Per EPAct 2005 requirements, GSA will eventually install advanced meters at all buildings where cost-effective.

- Integrate data from metering systems already in-place across GSA. ${ }^{12}$

Per EPAct 2005 requirements, GSA will eventually install advanced meters at all buildings where cost-effective. Applications are prioritized by the GSA regional managers primarily on the basis of several cost and usage metrics. Additional factors considered in the prioritization process include high-profile regional issues, current or planned use of energy savings performance contracts, multiple building facilities with shared heating and/or cooling plants, and verification of project savings. Funding for the meters and their connection to the agency software program is provided largely through the annual budget, although GSA seeks to leverage costs by also including meters as part of ongoing projects, as well as taking advantage of available metering offered by servicing utilities.

\footnotetext{
11 http://www.gao.gov/cgi-bin/getrpt?GAO-04-844.

12 There were already several metering systems in place within GSA when planning for the enterprise-based metering system began. These separate metering systems at the site and regional level may tap into additional functionality of the enterprise-based metering system should they so desire.
} 
Decisions on what meters to use are made by GSA regional staff. Data from the meters/sites are communicated to the centralized system through Modbus TCP/IP-compliant protocol. From there, the GSA metering system's enterprise energy management software provides the data storage, analysis, and reporting. This enterprise energy management software was developed under contract for GSA by a meter manufacturer, and resides on a server behind the GSA firewall. Figure 8.5 presents a sample output interface at one of the connected buildings.

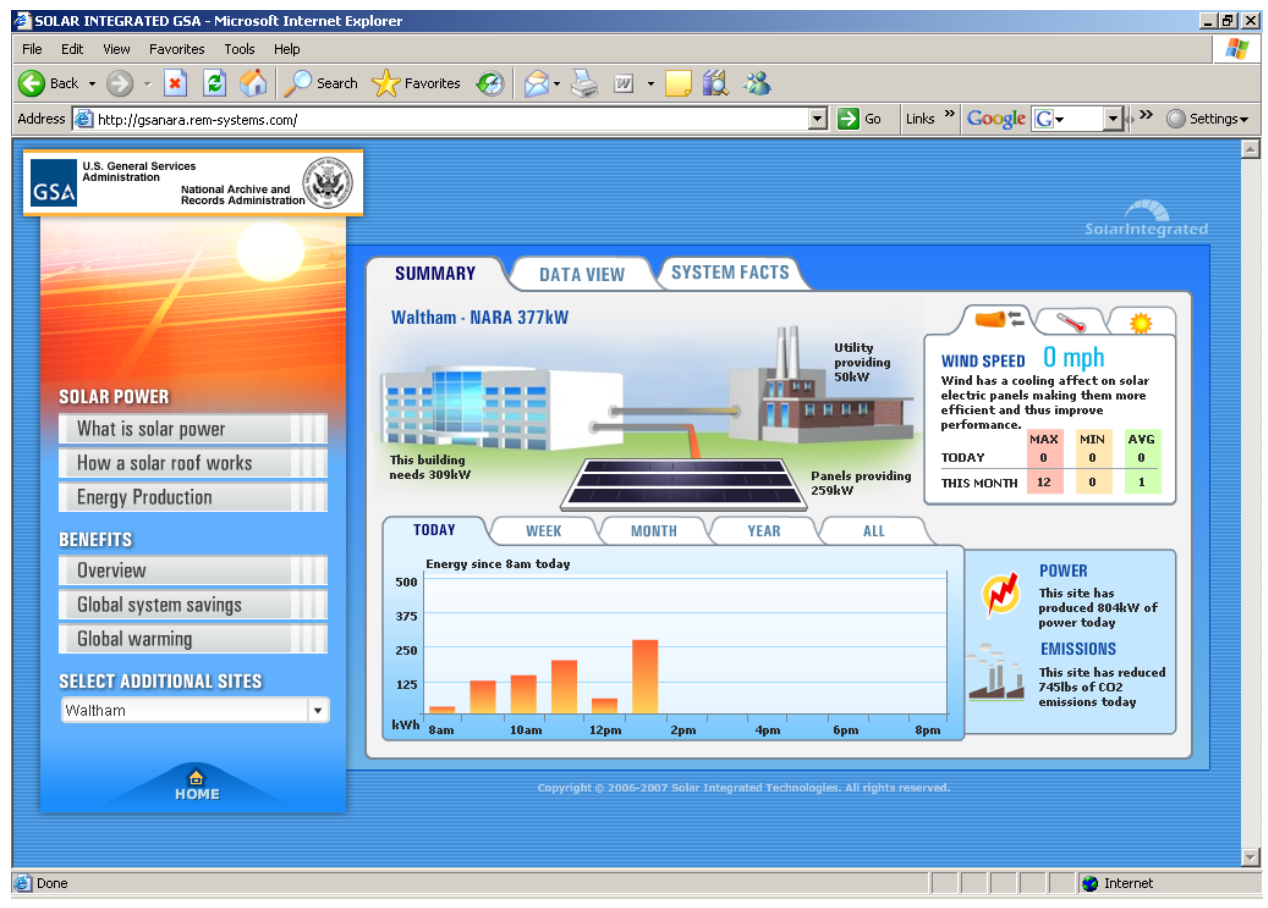

Figure 8.5. Summary of Current Conditions at a GSA Building in Waltham, Massachusetts. Data include current building demand, PV production, and utility supplied electricity. This website provides real 15-minute data.

The GSA's agency-wide metering system in 2007 was considered a work in progress as the installation of meters across the GSA building inventory and integration of the meters into the centralized system will take several years. Still, the GSA is able to cite some early uses and successes resulting from this program:

- Increased participation in voluntary demand-side load curtailment and price response programs, as well as contracted demand response programs (where agreed upon demand loads cannot be exceeded without incurring penalties).

- Development of data on energy savings realized in support of congressional testimony.

- Verification of ability to react to load and price conditions using metering technology and predetermined load shedding plans.

- Demonstration in real-time the actual production of electricity and resulting savings from a rooftop PV application in Waltham, Massachusetts. 


\section{Lessons Learned:}

- Centralized energy data use and reporting is a viable approach that can provide timely data at the site or headquarter level.

Centralized energy
data use and
reporting is a viable
approach that can
provide timely data at
the site or
headquarter level.

- Compliance with and administration of Homeland Security Presidential Directive (HSPD) 12 - Policy for a Common Identification Standard for Federal Employees and Contractors was challenging, and is credited with creating significant implementation delays. It is recommended that sites/ agencies understand agency HSPD 12 requirements and processes as they begin planning their metering systems.

- Large (nationwide) metering systems can create significant new system maintenance requirements. Support requirements from Information Technology staff needs to be identified up-front and budgeted.

- Interval metering at the building level allows GSA to increasingly participate in contracted electric demand management programs that carry greater financial incentives than the voluntary load curtailment programs. Participation in these programs is possible, in part, because there is an ability to better understand the load reduction commitment against current load requirements. Also, (near) real-time data make it possible for building operators to monitor their electric demand loads during the curtailment periods.

\subsection{National Institutes of Health Headquarters} (Leifer 2006)

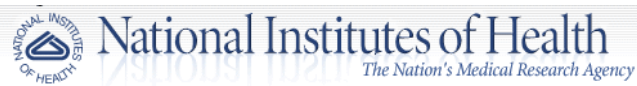

The National Institutes of Health (NIH) is the primary Federal government agency for conducting and supporting medical research. The NIH headquarters campus is located in Bethesda, Maryland. This campus includes over 10 million square feet of building space to support hospital, research, laboratory, industrial, and office uses. And as expected for a facility that houses medical research, the utility costs resulting from high-intensity uses are quite large: $\$ 75$ million in fiscal year 2005, and nearly \$95 million in fiscal year 2006. In an effort to better manage their utility costs, the NIH determined that a facility-wide metering program would be effective in

The National Institutes of Health metering system consists of over 350 meters. Over 200 of these are electric meters.
- Understanding utility use at the building level

- Monitoring and managing utility use and costs

- Diagnosing utility use problems.

The NIH metering system consists of over 350 meters. Over 200 of these are electric meters while more than 150 are non-electric meters for steam, chilled water, and domestic water. Figures 8.6 and 8.7 show NIH's chiller water metering and compressed air metering systems. Because of the size and complexity of the system, the purchase and installation of the meters is being done as part of a larger energy savings performance contract (ESPC). In this case, the energy savings from the meters are stipulated in the ESPC contract, as opposed to supporting specific energy conservation measures such as ongoing commissioning or real-time purchasing of electricity. 

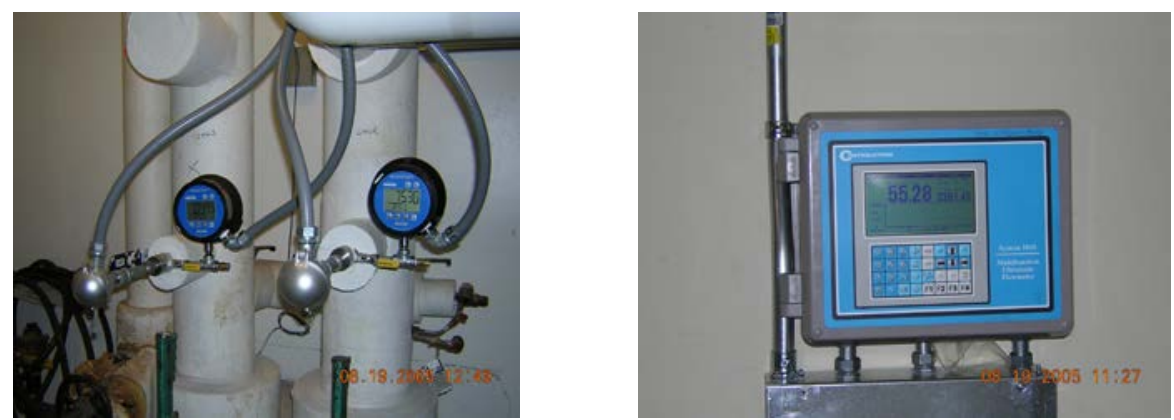

Figure 8.6. Chiller Water Metering at NIH. Photo on left shows water supply and return pressure sensors; photo on right is a chiller water meter energy/flow processor.
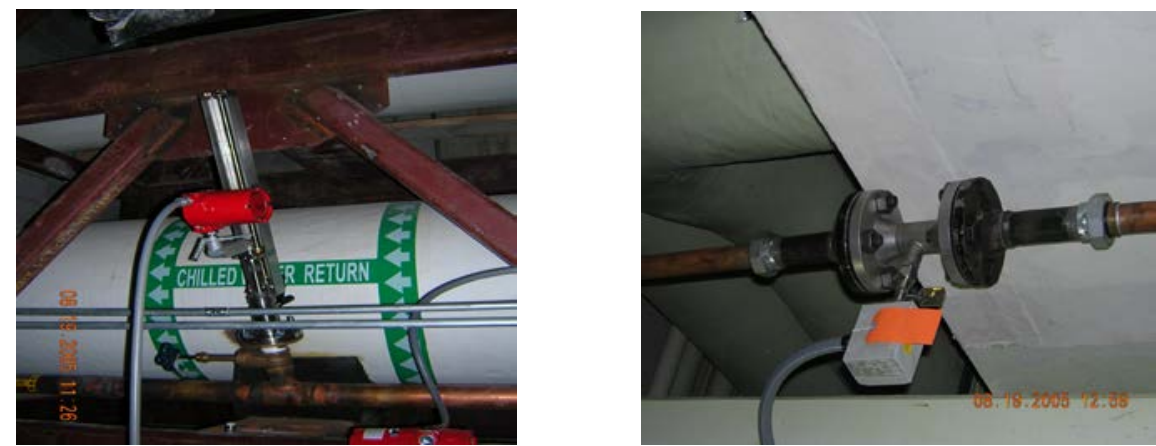

Figure 8.7. Compressed Air Meter Applications at the NIH. Meter in left photo is an insertion-type while meter in right photo is an in-line-type.

Features/characteristics of the metering system include the following:

- Electric meters chosen for application at substations were "higher-end" while "lower-end" electric meters were used at buildings.

- Insertion meters were used for steam, but ultrasonic meters were used for chilled water applications because of ease of installation (non-intrusive technology and piping bypasses not needed) and maintenance and calibration.

- Communications are handled through two different approaches:

- A separate SCADA system on a local area network is used for the electric meters

- The building automation system (BAS) is used for non-electric meters

- Data storage and analysis is done onsite.

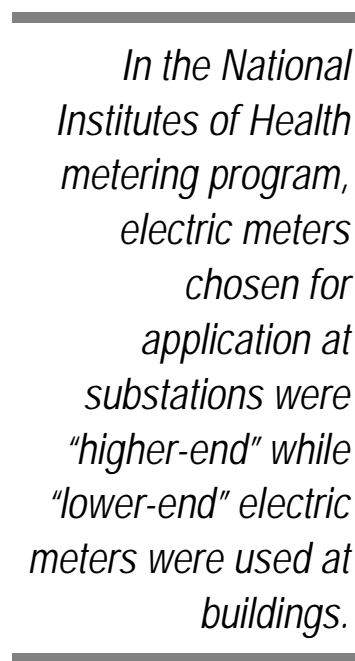

The installation of the metering system is not yet complete. However, Figure 8.8 demonstrates an early success of the metering program in helping to identify excessive steam use in a building. In this figure:

- the $\mathrm{x}$-axis is the time in increments of 1.5 hours (labels on 6-hour increments)

- the y-axis is pounds of steam per hour 
- the top line plot is the steam plant generation for the NIH campus

- the lower line plot is the steam demand for a single 270,000-square-foot building.

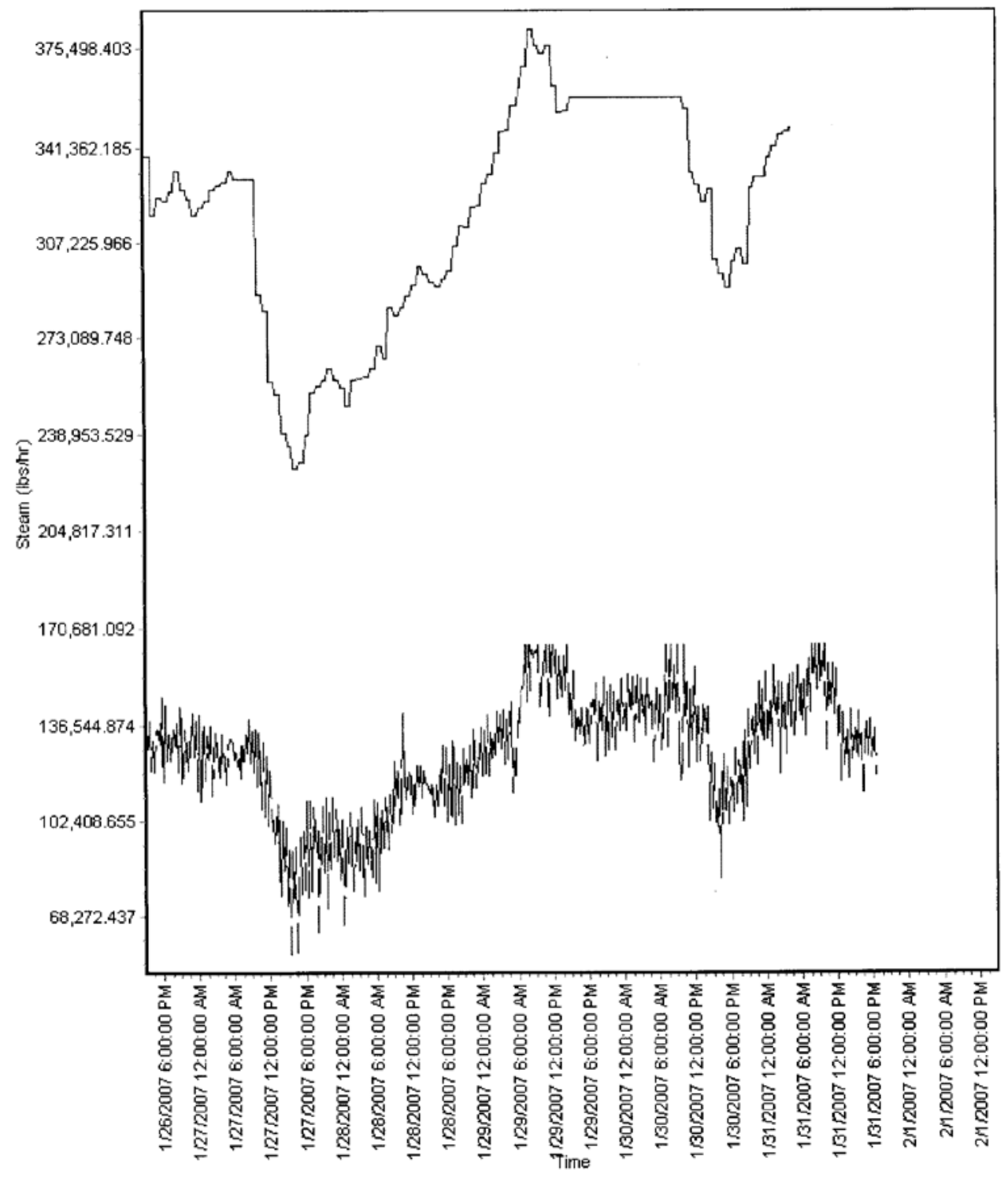

Figure 8.8. Total Site Steam General vs. Single Building Steam Demand

A quick review of Figure 8.8 shows that the single building steam use accounted for approximately 30 percent of the total site steam use, even though the building accounted for roughly 2.5 percent of the total site building square footage. The first action taken by the site was to verify the accuracy of the building steam meter. The NIH staff then inspected the building's steam systems and flow and found that the metered data appeared to be reasonable. The next step (currently ongoing) is to assess the building's systems, which indicates a likely preheating and controls strategy problem. At $\$ 10.00$ per thousand pounds of steam, the cost impact of poor operations such as this can become very expensive. 


\section{Lessons Learned:}

- ESPCs can be used to finance the purchase and installation of metering systems.

- It is cost-effective to combine the installation of meters for multiple utilities at the same time (as opposed to installing meters for one utility at a time).

- Correct design, specification, and installation are critical to the systems operating success.

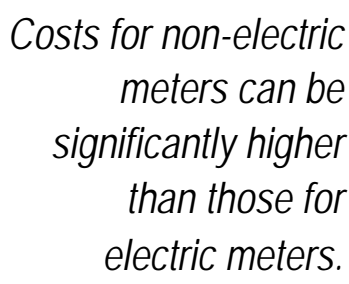

- Costs for non-electric meters can be significantly higher than those for electric meters.

\subsection{U.S. Department of Agriculture: Western Regional Research Center}

The U.S. Department of Agriculture (USDA) Western Regional Research Center conducts missionoriented research to enhance the healthfulness of foods, to develop new food and industrial products from renewable resources, and to protect and enhance the quality of the environment. The results of these research efforts are implemented through the effective transfer of new and innovative technologies to appropriate clients and users. Concurrent with this mission, is a focus on energy use and efficiency within the variety of buildings and research facilities on the campus. To those ends, USDA facility engineers embarked on an intensive building-level metering effort to both satisfy internal needs for energy use tracking/efficiency and comply with requisite Federal metering legislation.

The Western Regional Research Center is located in Albany, California. This location is home to 23 buildings, 312,700 square feet, providing research facilities that serve 300 staff. The site has average electric energy-use intensity (EUI) of $38.1 \mathrm{kWh} /$ square foot. High-energy intensity areas include greenhouses that have artificial lighting sources, chemical research laboratories that use 100 percent outside air, and a small data center.

\subsubsection{Metering Objectives}

At the outset, the goals of this metering project were to comply with legislative requirements of EPAct 2005 and EISA 2007, and to identify high usage patterns of buildings and systems leading to energy usage and cost reductions.

\subsubsection{Approach}

As an overarching approach to this activity, the staff at the Research Center wanted to focus on making use of existing infrastructure as much as possible - the rationale for this was two-fold. First, this eliminated the need for burdensome shutdowns of the building electrical systems to safely retrofit new instrumentation. Second, pure economics - anytime you can make use of existing systems and equipment (provided they will meet objectives) the overall economics of the program typically improve.

For this particular activity, the metering planning focused on:

- Circuit-level sub-metering within the three distinct wings of the campus’ primary laboratory building, which are known to have a high-energy intensity. 
- Deployment of added I/O (input/output, both analog and digital) at the metering devices in the central plant building, with the intention of capturing campus-level natural gas usage data (at a minimum).

- Planned efforts to further sub-meter specific tenant-occupied areas, for detailed cost allocation.

- Better enable an investment-grade audit required for a planned financed energy savings project, along with further allowing detailed measurement and verification (M\&V).

- Deployment of the area-wide metering database.

\subsubsection{Implementation}

Once the planning phase was completed, attention turned to implementation. For this specific activity, all equipment and services were successfully acquired from the GSA Schedule. ${ }^{13}$ Included as part of this award was all capital equipment and the following:

- Turnkey installation (supply through commissioning) of all metering, wiring, and instrumentation in the switchboards.

- Software with an underlying SQL database, including SQL license and metering device licenses.

- All network drawings.

- Point-to-point wiring was separately awarded to the in-house maintenance contractor.

\subsubsection{Meters}

The site has installed a variety of meters at substation, panel and end-use. This collection used existing wiring, enclosures, and other infrastructure whenever possible.

- One advanced electric meter was installed at the service entrance with an onboard web server, in the utility's metering cabinet, “jumpered” off the utility's test switch. The meter socket base was installed by the local utility, at a cost that was additionally funded through this project.

- Six advanced meters with web-enabled gateways were installed at each low-voltage substation, with an ability to connect downstream sub-meters. These meters fit into the existing openings of the switchboards, without having to cut the sheet metal, and leveraged existing instrument transformers.

- One virtual meter was created to monitor a hard-to-reach low-voltage substation, which allowed for trending a mathematically derived $\mathrm{kW}$ and $\mathrm{kWh}$ value for this substation. The calculation was a simple subtraction of all other low-voltage substation energy from the campus-wide energy monitored at the medium-voltage service entrance.

- Twenty-four sub-meters, reporting a relatively broad level of data including kilowatts, power factor, and total harmonic distortion, are connected to almost all of the feeder breakers serving various subpanels and motor control centers in the main building to provide the next level of energy monitoring.

Figure 8.9 presents the metering configuration, highlighting the metering points relative to the service connection.

${ }^{13}$ GSA schedule relevant to meters and metering equipment can be found at: http://www.gsaelibrary.gsa.gov/ElibMain/sinDetails.do;jsessionid=33D297E35F7DB57A5205FA11FEEC6B46.nod e2 ?executeQuery=YES\&scheduleNumber=56\&flag $=\&$ filter $=\&$ specialItemNumber $=412+17$. 


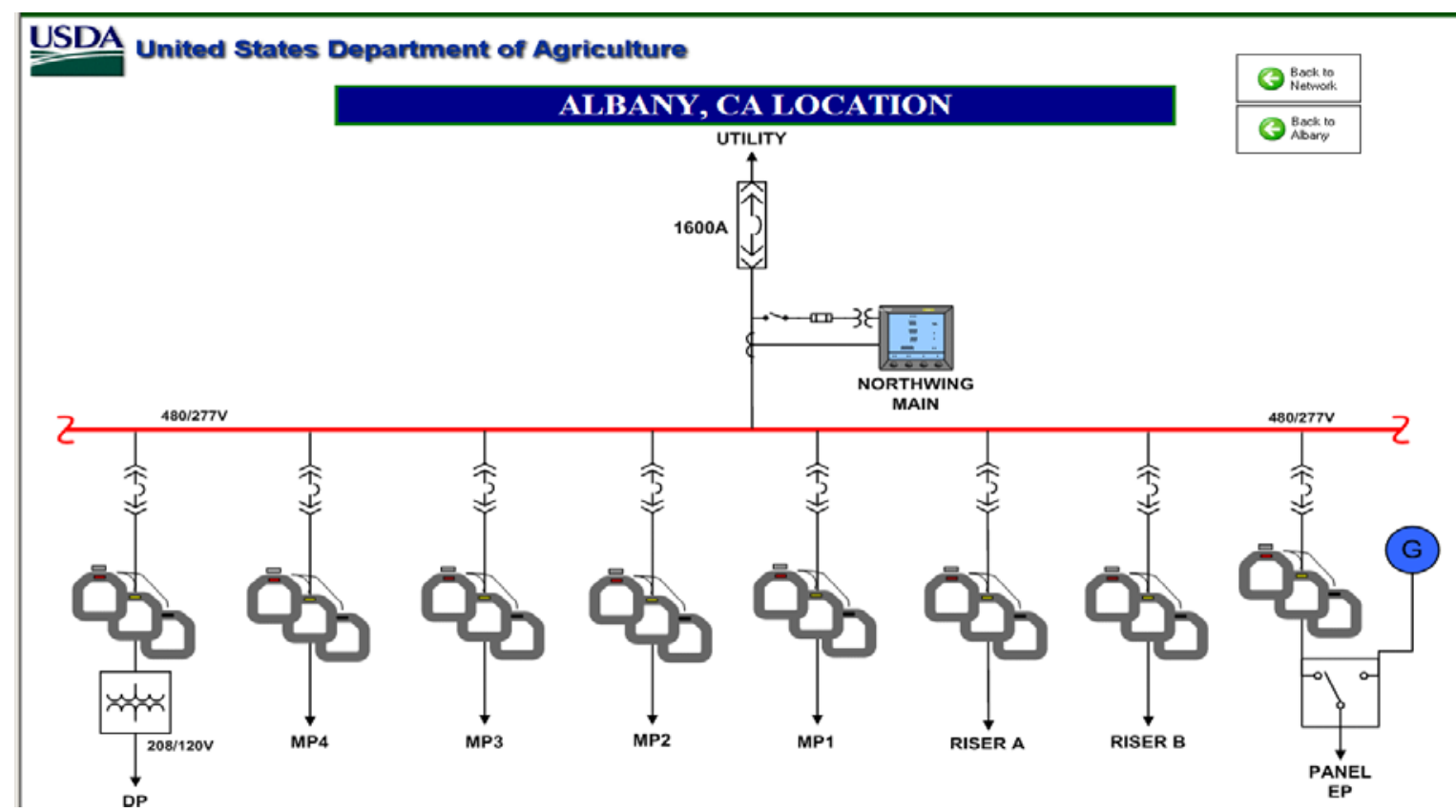

Figure 8.9. Sample of Meters Shown in Graphical User Interface

\subsubsection{Communications}

The point-to-point wiring includes a novel design proposed by the local IT staff including partial use of existing telecom wiring infrastructure through a technology called VDSL (Very high bit-rate Data Subscriber Line) which allows very fast data communications over copper lines - in this case, existing copper lines. The benefits to this approach included:

- Minimized additional cable runs, which for CAT5 is limited to 350 feet

- Leveraged existing wiring and termination cabinets that reached remote buildings without the added expense of wiring capital and installation costs.

\subsubsection{Data Storage and Processing}

Data is collected and stored on a quad-core Xeon processor server with $178 \mathrm{~GB}$ of hard drive space. This server is also houses the structured query language (SQL) database used for data processing. This simple database structure allowed for easy access by the organization's higher-level energy analysis database, which enabled additional plotting, benchmarking, and trending within an environment that contained real property data such as square feet, and utilities billing data such as power factor charges.

\subsubsection{Findings}

All metered data are collected and processed using Talisen's Enterprise Sustainability Platform (www.talisentech.com), which also houses the utility bills and can further process Building Automation Systems (BAS) data. These data are then used to develop time-series chart of relevant parameters. Examples of the findings are provided in Figures 8.10 through 8.12. 


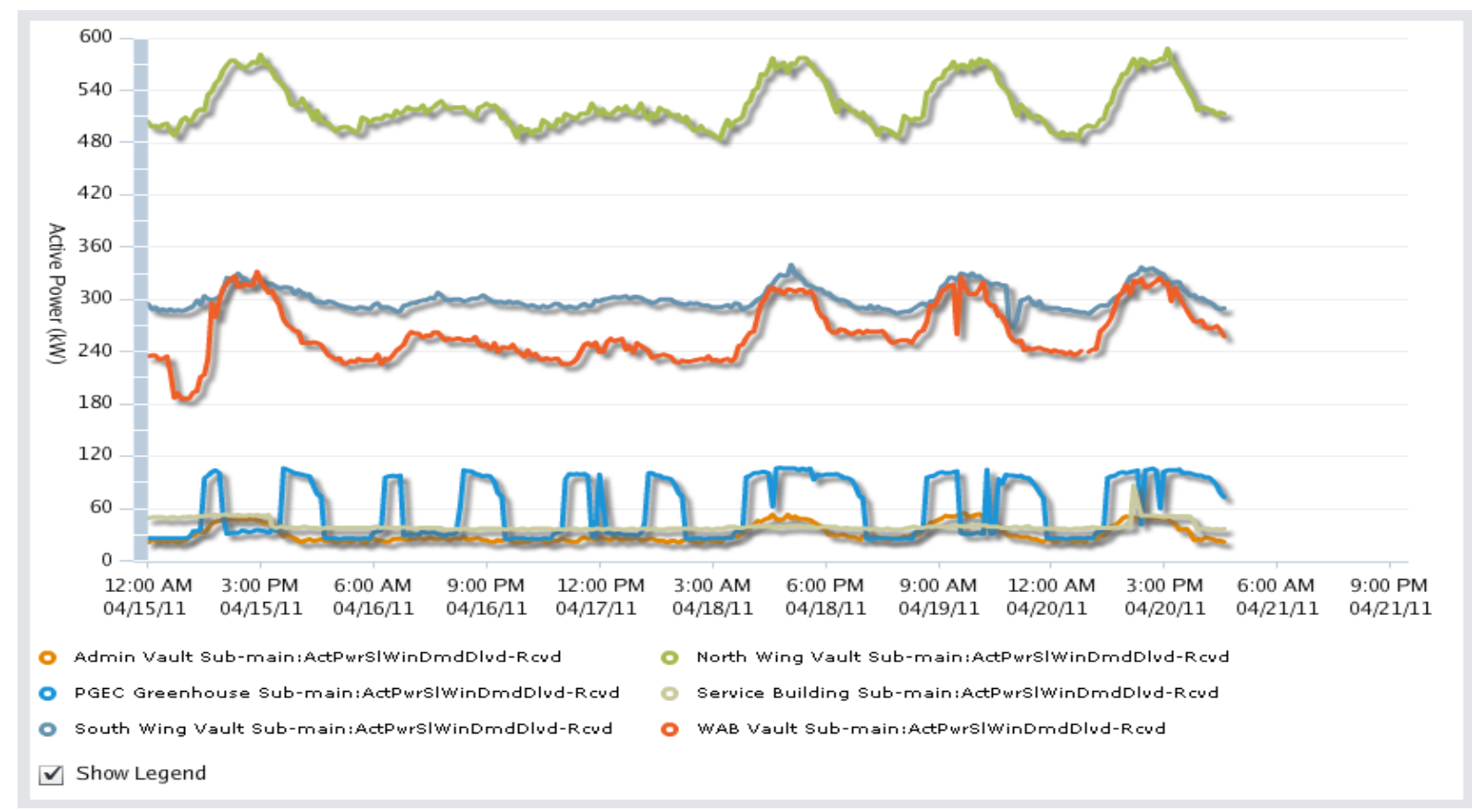

Figure 8.10. Time Series Power (kW) Profiles of Laboratory (top three lines) and Greenhouse (bottom blue line) Facilities for the Period Friday, April 15, 2011, through Wednesday, April 20, 2011. Note the reduced laboratory power draw on weekend (April 16-17) and consistent (cyclic) power draw of Greenhouse Complex.

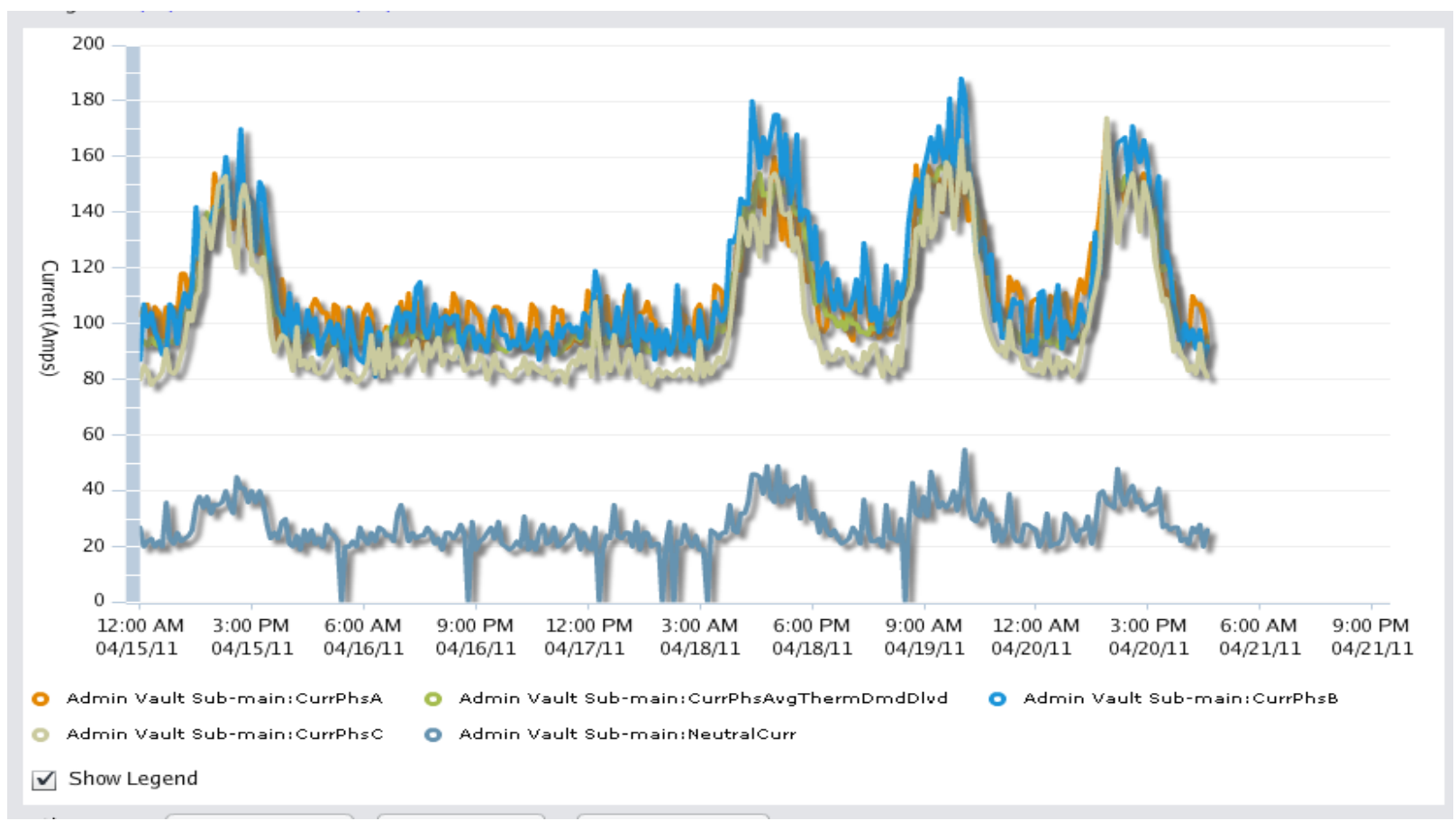

Figure 8.11. Three Phase Amperages - Phases A, B, C, and Average (top four lines) and System Neutral for Office Wing. Data highlight the phase and neutral load are well within the switchboard's 2000A rating (100 percent rated neutral). 


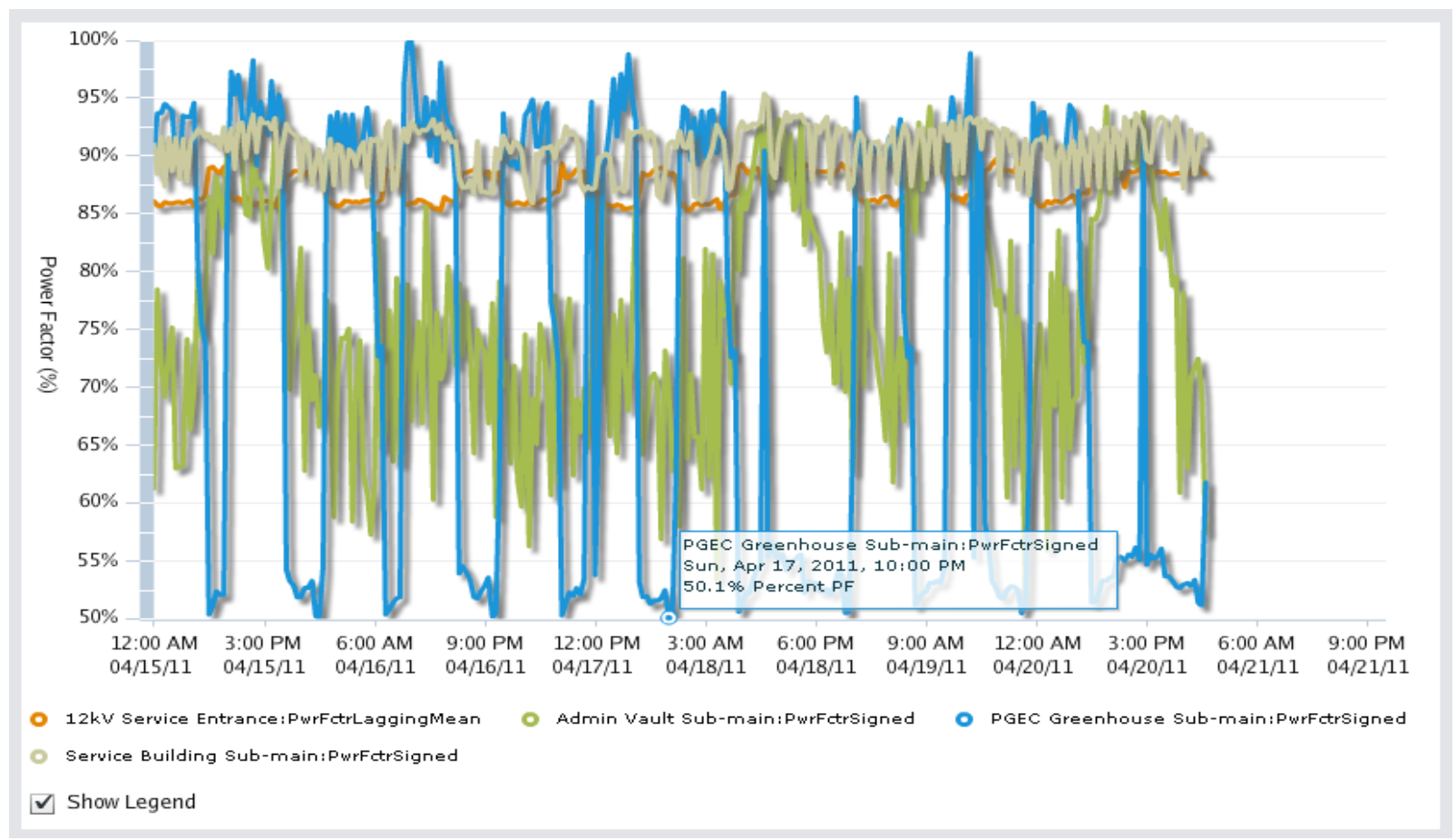

Figure 8.12. Time Series Power Factor (PF) Profiles of Service Main (Orange Line), Admin Wing (olive line), Greenhouse (blue line), and Service Building (gray line). Note the relatively poor PF of the Admin Wing and wide fluctuation of PF with the Greenhouse.

\subsubsection{Lessons Learned}

This project earned extra value by making use of existing infrastructure - this allowed a limited metering budget to be stretched to include additional components and metering points. The system was used to monitor circuit capacity in a wing under current renovation. The original load-flow study from the architect-engineer suggested a given circuit was at capacity and should be increased during the renovation, at a cost of $\$ 200,000$. Using the monitoring of that circuit for over one year, the actual measurements confirmed the estimate was very conservative, and that there was excess circuit capacity. However, since the appropriated construction funds could not be re-purposed, the decision was made to stick with the originally planned circuit modifications to ensure ample capacity as future reserve.

The initial metering contract to accomplish this work was canceled for a failure to address "issues of outages/downtime." This initial contract included supply and installation of only four low-tech meters, and one software CD that could be loaded onto a laptop to temporarily connect to the meters. Installation details were not made clear. The revised contract, using the Federal Supply Schedules with the same budget, included the supply and installation of the following:

- (1) Highly advanced meter at the service entrance with an onboard web server, which would not have been previously metered.

- (6) Advanced meters with web-enabled gateways for connecting to downstream sub-meters. Two of the locations would not have been previously metered, and through the advanced features of the 
selected software a 3rd "virtual" meter will be added to represent the lone sub-main location that will not be monitored due to physical constraints.

- (24) quality sub-meters, still including a relatively broad level of data including total harmonic distortion, which would not have been previously metered.

- (1) Server with Quad-core Xeon processor and 178 GB of hard-drive space for long-term data storage.

- Point-to-point wiring of all necessary communications devices between the meters and the server, which would not have been previously included.

As with any project involving installation in and around energized components, there is always the possibility of an incident affecting the safety of persons and property. During installation, an electrical "arc flash" originated at the metering cabinet in an electrical room. Fortunately, the contractor was known to have an extensive arc flash safety program, including the requisite personal protective equipment (PPE). Because of this program, and its adherence to by the installation staff, the installer was not hurt.

\subsection{NASA Marshall Space Flight Center Metering Program}

Established in 1960, the National Aeronautics and Space Administration's (NASA) Marshall Space Flight Center (MSFC) has a longstanding, successful sustainability program that focuses on energy and water efficiency as well as environmental protection. MSFC's key operations include propulsion and transportation systems for the space shuttle and development of NASA's new heavy lift launch vehicle system. MSFC also provides advanced engineering and operations for International Space Station systems. Located in Huntsville, Alabama, a tenant of Redstone Arsenal, MSFC has more than 4.5 million square feet of building space occupied by 7,000 personnel. MSFC consumes approximately 240 million gallons of potable water annually and 124 million KWh annually in electricity annually.

\subsubsection{Program Description}

MSFC has an extensive campus-wide metering system that monitors consumption of electricity, chilled water, natural gas, steam, potable water, industrial water, and wastewater discharge. There are 308 electric meters installed at the building level that serve over 95 percent of the floor space at MSFC. MSFC has a central chilled water plant that has five metered loops which provides chilled water to 3.7 million square feet of the campus. A majority of the chilled water is also metered at the building level. These meters read the temperature drop between the inlet and outlet of the building along with the flow rate to measure the energy consumed by the building in chilled water. In addition to the extensive electric and chilled water metering, MSFC also monitors other utilities at the building level including 39 steam meters and 34 potable water meters. Individual processes are also metered such as make-up supply on the cooling towers, natural gas consumption of boilers, hot water consumption, and landscape irrigation water used on the ball field located at the center.

Data are collected from all meters and managed in a central building automation system that is used to analyze consumption and trends at MSFC. These data are reported to NASA Headquarters on a quarterly basis, which satisfies Federal reporting requirements. Locally, the data are used in a variety of ways. Anomalies are identified in the data that may indicate operational problems. Also, data are used to benchmark facilities and prioritize buildings for auditing and efficiency improvements. For example, 
each month the top 15 energy-consuming buildings in electricity are identified, which allows the MSFC energy and water management team to identify which buildings represent the biggest load and then the team compare these values to previous years to see if any major shifts in energy use have occurred. This information is used to prioritize building retrofit projects. Building-level metering is also used to indicate actual savings of efficiency improvements by monitoring building pre and post retrofit usage. This type of data management and analysis provides essential information for strategic planning for water and energy initiatives at MSFC.

\subsubsection{Success Story}

MSFC identified an operational malfunction of a cooling tower through the cooling tower make-up water meter. MSFC's metering program monitors and archives cooling tower make-up water on a continual basis. In the summer months, higher make-up water is expected because of large cooling loads. In the winter months, it is expected that the volume of make-up water drops because of cooler weather. During January and February of 2011, the metering team noticed larger than normal make-up water consumption totaling about 4 million gallons each month. This water use was 2 million gallons higher than the average of the previous three year's winter time measured use.

This issue triggered an investigation of the cooling tower system. The facility engineering team discovered the basin that collects the cooled water to be sent back to the chiller was filling to levels above design. As a result, water was constantly overflowing into the sanitary sewer by means of an overflow drain that was hidden from view. This was caused by a malfunctioning make-up water valve. This butterfly-type valve was not properly closing and was allowing between 30 to 50 gallons per minute of water to constantly flow into the basin and causing the basin to overflow. By repairing the malfunctioning valve at the onset of the problem, MSFC saved at least 1 million gallons and nearly $\$ 3,000$. But if the problem would have persisted and the valve not repaired, the issue could have spilled over into the following winter creating additional losses of 11 million gallons and over \$27,000 in water and wastewater charges annually.

Figure 8.13 is an output from the MSFC metering system that reveals the high water usage during January and February of fiscal year 2011 compared with other fiscal years and then shows a subsequent drop in water use in March after the valve was repaired.

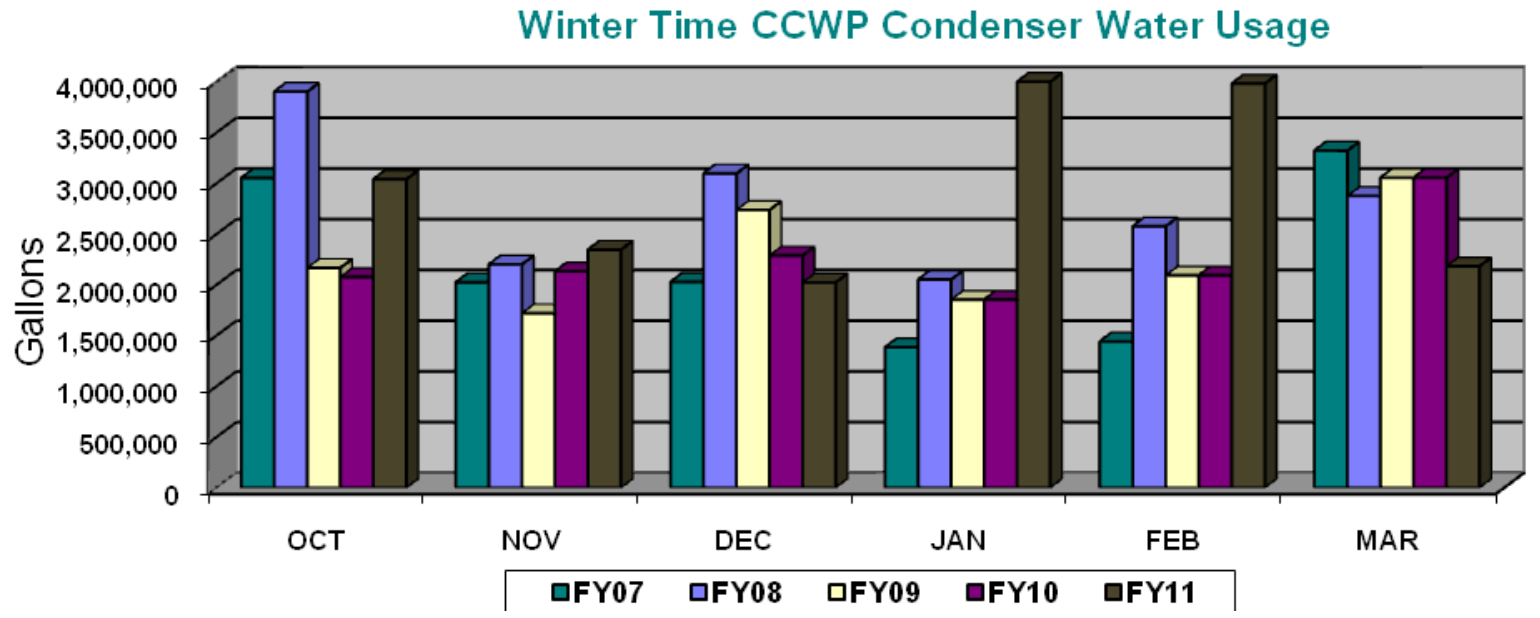

Figure 8.13. Winter Time CCWP Water Consumption FY 2007 through FY 2011 


\subsubsection{Future Enhancement}

A noteworthy project that is currently under development at MSFC is a potable water metering system. MSFC receives potable water from Redstone Arsenal and the city of Huntsville and is billed directly from the Redstone Arsenal for water from either source. MSFC began noticing large fluctuations in monthly water charges from Redstone Arsenal, but had no way to verify the accuracy of the bills. So MSFC installed bi-directional flow meters on all entry and exit points of the potable water system at the MSFC/Redstone Arsenal boundaries. The meters communicate through a wireless connection to the building automation system. Meters in remote location are solar powered.

Once properly commissioned, the metering system will allow MSFC to verify the billing data from Redstone Arsenal and also identify other potential issues. For example, thresholds of normal will be programmed into the system; if these thresholds are exceeded facility management will be notified to investigate the issue. Alarming systems such as these become very useful in identifying leaks and other operational problems resulting in inefficient operation or resource loss. A similar metering system is being implemented on the electric feeds into the center as well.

\subsection{U.S. Coast Guard - Baltimore Yard Electric Metering Program}

For over a century, the United States Coast Guard Yard has built, repaired, and renovated ships in Baltimore, Maryland, for the U.S. Coast Guard. The Baltimore Yard is the Coast Guard's sole shipbuilding and major repair facility, and an essential part of the Coast Guard's core industrial base and fleet support operations.

The site encompasses over 100 acres with 60 buildings including significant marine and ship building/ repair facilities, industrial shops, administrative buildings, and a variety of craft-service facilities. In addition, the Coast Guard Yard has over 30 shore ties providing utility services to ships at dock. The site buildings encompass 980,000 square feet with annual utility bills averaging $\$ 1.5$ million for electricity and $\$ 600,000$ for natural gas. Figure 8.14 provides an aerial view of the site.

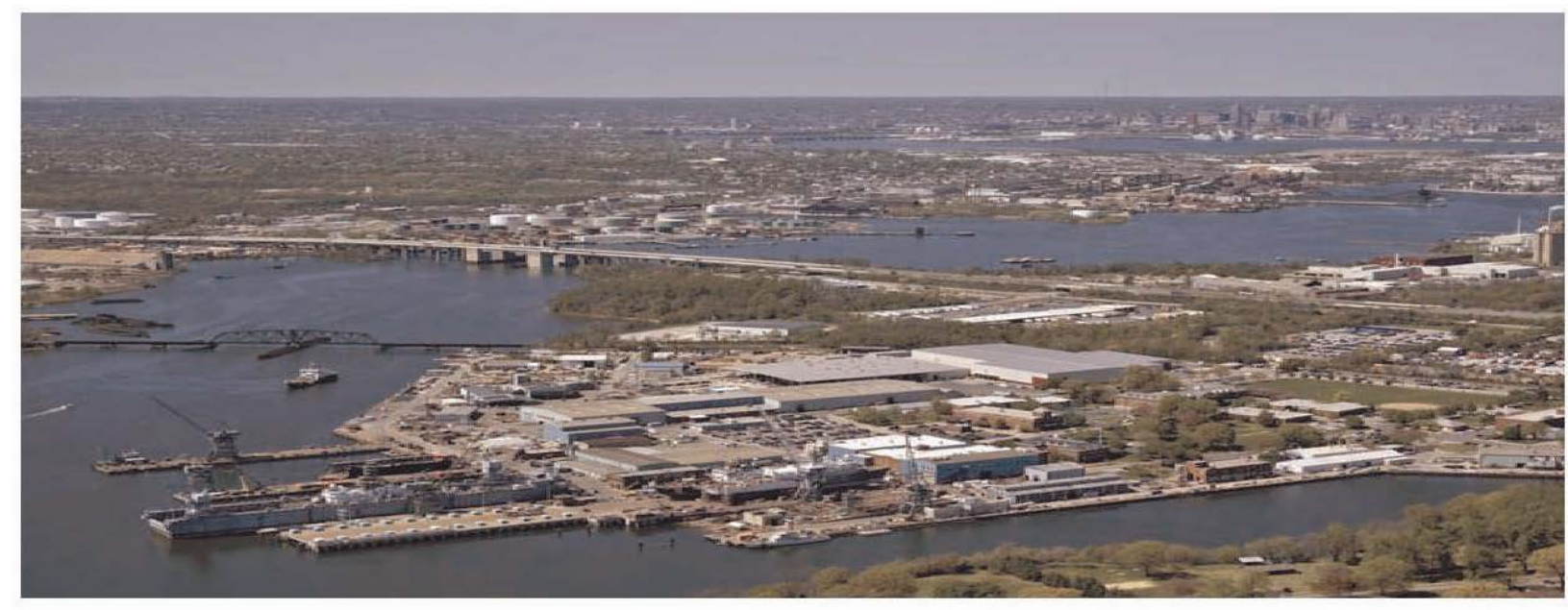

Figure 8.14. The Coast Guard Yard at Baltimore, Maryland 


\subsubsection{Metering Objectives}

At the outset, the goals of this metering program were to pilot electric metering systems while carefully documenting cost, application challenges, and ultimate benefits. To start, three systems were identified for metering:

- 300-HP industrial air compressors

- Warehouse lighting

- Electric shore-ties providing power to ships at dock.

These three systems were specifically chosen because of their suspected high energy use and the lack of information on actual use.

\subsubsection{Approach}

As an initial approach, site facility staff spent time to clearly define the energy metrics of importance and how those metrics can be implemented while keeping within tight budgetary constraints. From an energy and resource management perspective, the following list of metrics was developed as being applicable for most facility/system metering needs:

- Kilowatt $(\mathrm{kW})$ demand

- Kilowatt-hour (kWh) use

- Cubic feet of natural gas

- Pounds of steam

- Tons of chilled water.

While other metrics were considered (e.g., power factor, power quality, harmonics), the added expense to meter with these capabilities was considerable. In addition, these types of measurements are not often needed and when they are can be accommodated with portable and/or temporary metering equipment.

Once the desired metering metrics were identified, the activity shifted to understanding the available equipment in the market. Before deciding on vendors, the staff identified four different categories of metering and communications system approaches offered by prospective vendors; these included:

1. Proprietary systems - meters, communications, and software making use of vendor-specific technologies and protocols.

2. Generic meters/proprietary communications and software - standard meters connected to proprietary communications and software systems.

3 Generic meters and standard communications with proprietary software - standard meters and communications operating with proprietary software.

4. Open architecture - meters, communications, and software that are completely open and nonproprietary. 
While a valid case can be made for any one of the above options, the Coast Guard Yard decided that having an ability to change vendors in the future, make use of both wired and wireless open protocol communications, and allow for the porting of collected data to and from different software systems, all had great value. Option 4, open architecture, was its choice.

\subsubsection{Implementation}

Once the pilot planning phase was completed, attention turned to implementation. For this specific activity, all equipment and services were successfully acquired from the GSA Schedule. As part of the pilot, the systems each were metered with three-phase watt transducers, a wireless communications system, and a single centrally-located data logger. A benefit to this system is that all parts are open architecture and inter-operable (i.e., they function using non-proprietary communication modes).

- Meters - True power is measured via the three-phase power meters, which have pulse or RS 485 (also known as Modbus) outputs - both non-proprietary communications protocols.

- Communications - Data are sent via both wired and wireless networks to a centrally located data logger. The wired option can have proximity and logistical constraints. The wireless option uses a series of radios configured in a mesh-network to optimize data collection and transfer. Wireless can also have logistical challenges; however, through the use of repeaters and properly configured mesh "nodes," these can usually be overcome.

- Data Collection and Storage - Data are collected and stored by a data logger with web-server capabilities. This logger allows network connectivity (automatic data uploads to fee-based webhosting site) or remote connection (web- or modem-based). In either case data can be uploaded at prescribed intervals or viewed in real time.

Figure 8.15 presents a graphical representation of this metering network as it applies to three connected Coast Guard facilities.

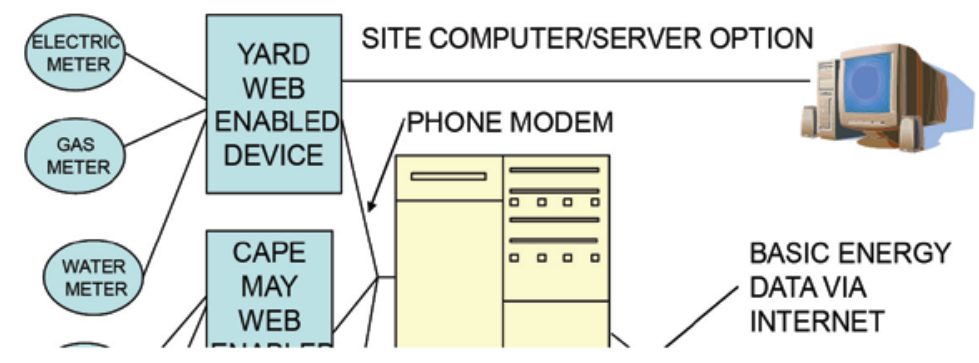

Figure 8.15. Resource Metering Configuration at Three Coast Guard Installations (Baltimore Yard; Cape May, New Jersey; and Cape Cod, Massachusetts) 


\subsubsection{Findings}

Figures 8.16 through 8.18 highlight a sample of the findings from the pilot metering efforts at the Coast Guard Yard. Evident from these graphs are significant savings related to equipment operation and nighttime shutdown. Figure 8.16 shows time-series power draw for a 300-HP industrial air compressor; significant savings were achieved from implementing a night shutdown strategy.

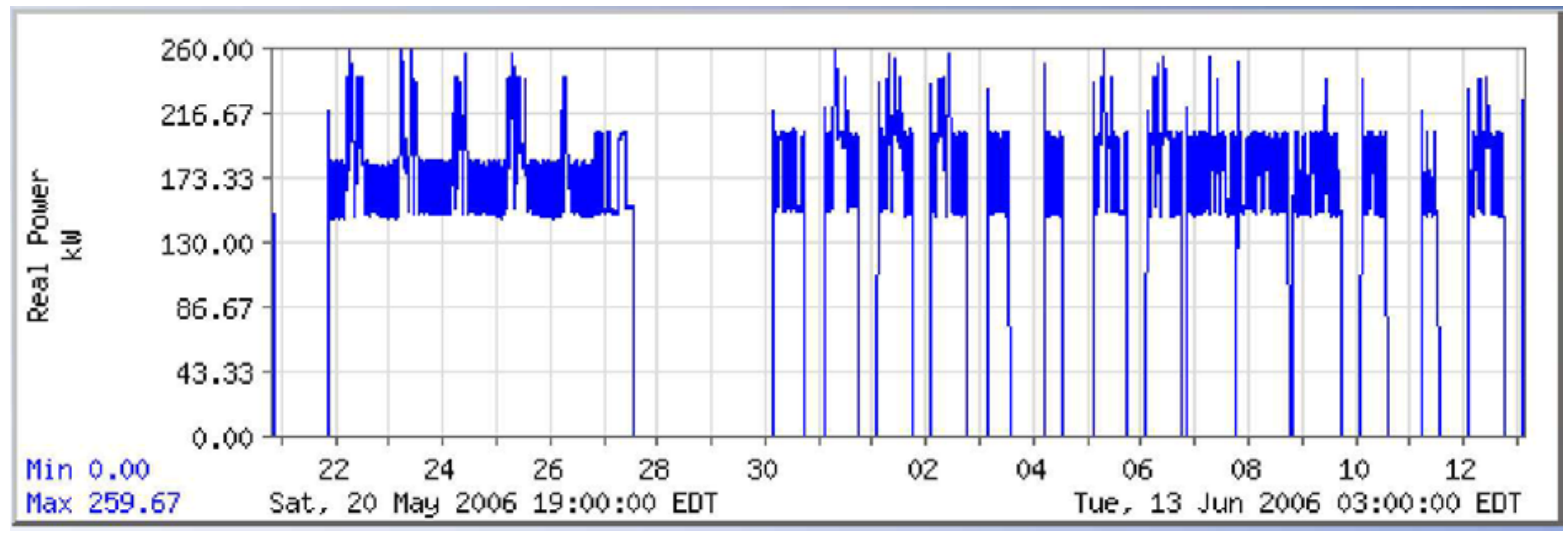

Figure 8.16. Air Compressor Operation "Before” (left of break) and “After” (right of break) Metering Program Implementation. Note the profile change from 24-hour operation to shift operation.

Figure 8.17 presents the time-series lighting power draw for a large warehouse before and after a night shutdown strategy was implemented. As shown, roughly $55 \mathrm{~kW}$ was saved when the strategy was implemented lasting approximately 12 hours per day, for a savings of more than $600 \mathrm{kWh}$ per day.

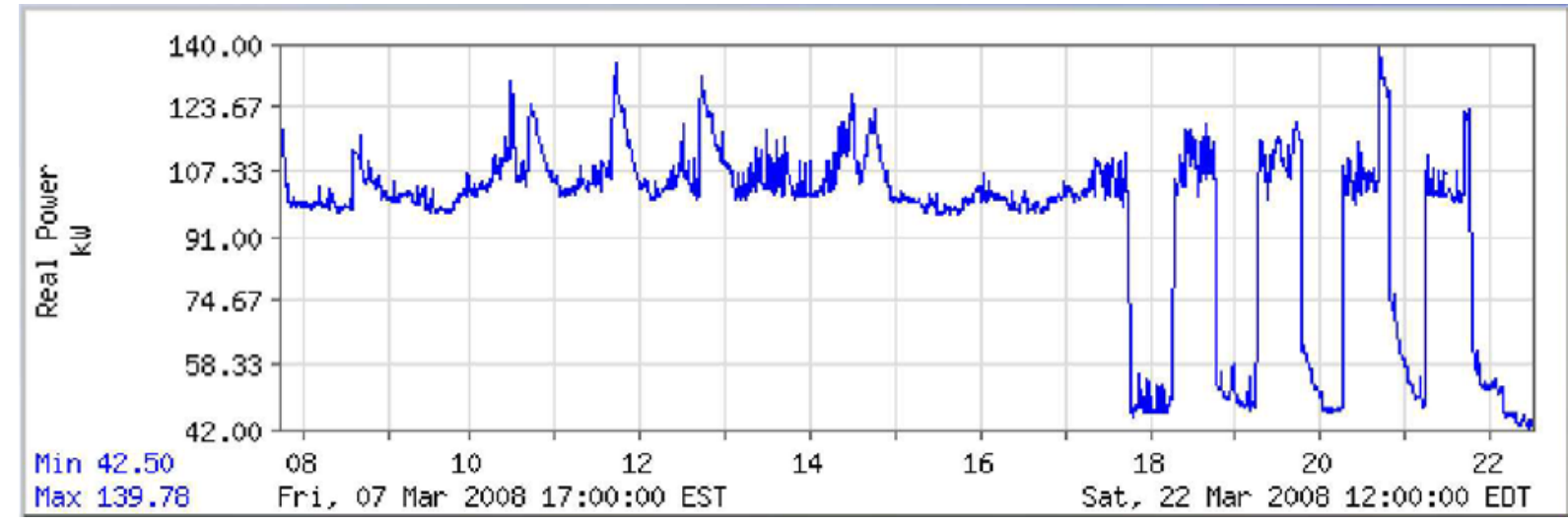

Figure 8.17. Warehouse Lighting Operation "Before” (flat portion of graph) and “After” (cyclic portion of graph on right) Metering Program Implementation. Note the profile change from 24-hour operation to shift operation.

Similar to the air compressor and warehouse lighting, Figure 8.18 details a night shutdown strategy for an electric welder whereby $55 \mathrm{~kW}$ is saved through improved operation. 


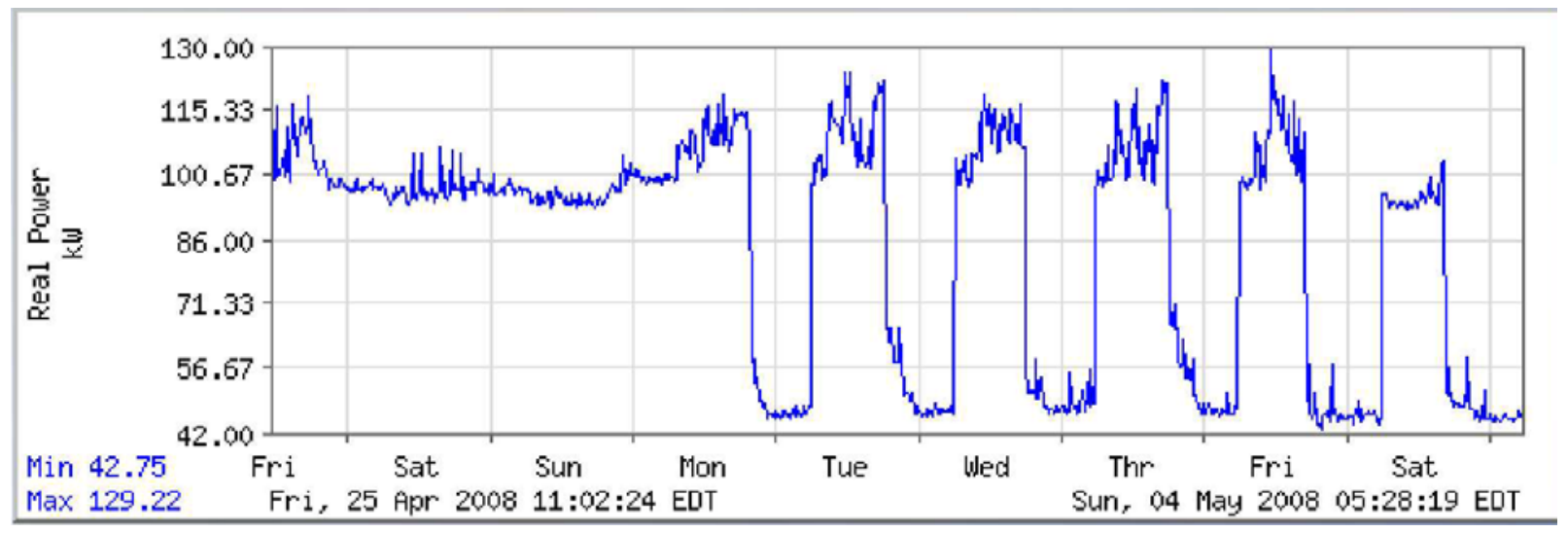

Figure 8.18. Electric Welder Operation "Before” (flat portion of graph - upper left) and “After” (cyclic portion of graph on right) Metering Program Implementation. Note the profile change from 24-hour operation to shift operation.

Metering costs can vary greatly depending on system configuration, installation and infrastructure needs, and communication options. On a per point basis, the metering system was typically installed in less than 1 day at a cost of under $\$ 2,000$. Based on the data presented in Figures 8.15 through 8.17 with an installed cost of $\$ 2,000$ and an average savings of $\$ 50$ per day or $\$ 1,500$ per month, the payback would be under 2 months.

\subsubsection{Lessons Learned}

Metering at the Coast Guard Baltimore Yard has resulted in significant energy and cost savings opportunities. From the Coast Guard's perspective, the lessons to be shared included the following:

- Start with the end in mind. At the onset, each project should first answer the basic question: Why metering? Define the metering objectives and how the data generated will be used to meet those objectives.

- Choose equipment appropriate for the project. When choosing metering equipment, consider the basics. Resist the temptation to "over-buy”; rather, focus on the metrics needed to accomplish your objectives.

- Start small. Consider starting with a smaller demonstration of a larger project. Use this demonstration to test different technologies, manufacturers, and installation techniques. Identify what work well before moving forward with the larger implementation.

- Understand relevant technology options. In the case of the Baltimore Yard, making use of wireless communication technology resulted in large installation cost savings.

\subsection{Department of Veterans Affairs Advanced Metering Program}

The Department of Veterans Affairs (VA) was originally established in 1930 as the Veterans Administration when Congress authorized the President to "consolidate and coordinate Government activities affecting war veterans.” The VA health care system has grown from 54 hospitals in 1930 to over 170 hospitals and medical centers; more than 350 outpatient, community, and outreach clinics; 
126 nursing home care units; and 35 domiciliaries. The total VA portfolio, which includes national cemeteries and benefits administration offices as well as health care facilities, encompasses 155 million square feet and approximately 6,000 buildings. Figure 8.19 presents a typical VA medical center.

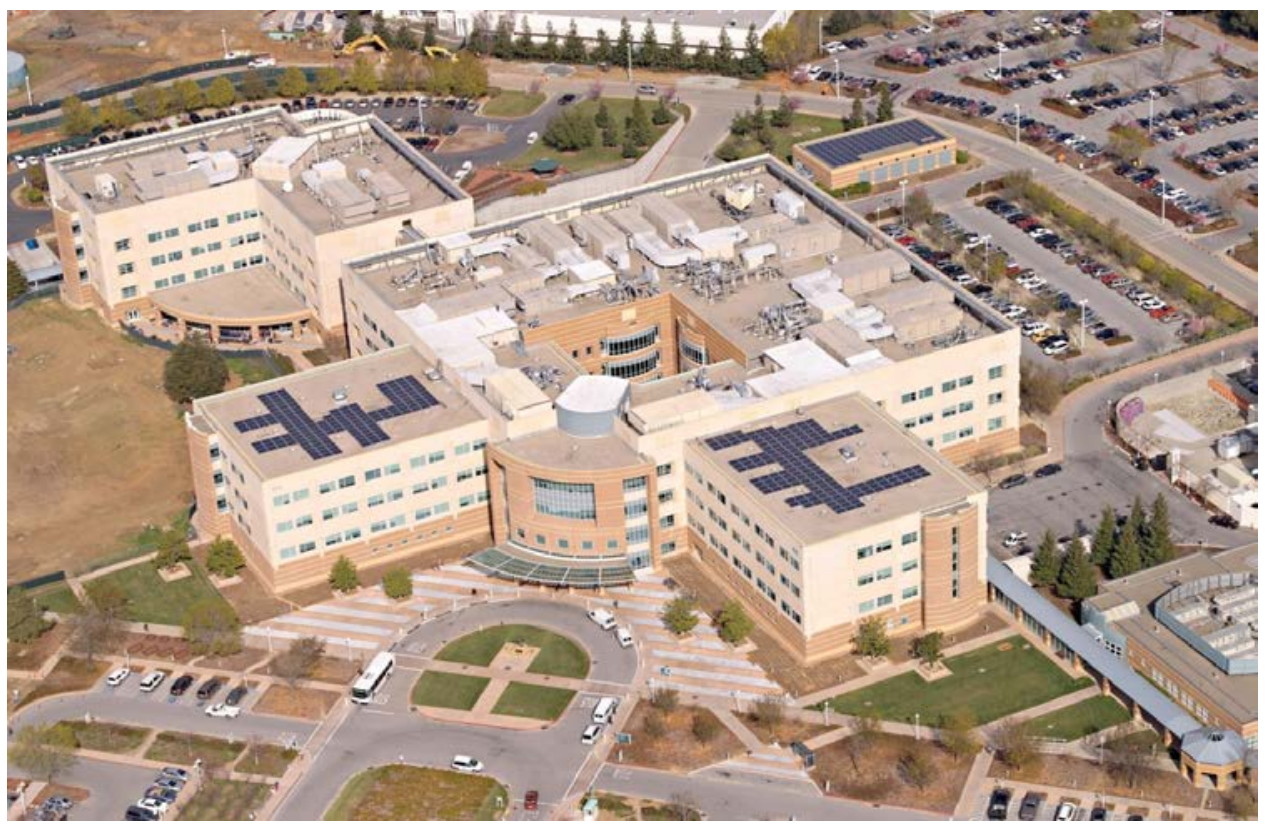

Figure 8.19. Veterans Administration Medical Center, Palo Alto, California

From a resource use perspective, in FY 2009 the VA used 29 trillion Btus of energy and 9 billion gallons of water; this resulted in expenditures of \$517 million on energy and \$31 million on water. These expenses represented a 78 percent increase over FY 2003 levels. Faced with these escalating energy/ water bills, aging infrastructure, and the need to respond to Federal mandates, the VA initiated an aggressive advanced metering program.

\subsubsection{Metering Objectives}

The VA's objectives were to enhance local and agency level energy management programs and initiate a higher level of resource accounting through the detailed metering of electricity, natural gas, steam, chilled and potable water. Energy managers at hospitals, regional offices, and administrations monitor and analyze metered data for enhanced energy programs. These data allow managers to quickly identify problems in a specific building, such as water leaks and deficiencies in HVAC controls. Another critical component of this resource accounting is the future ability to identify cost-effective energy and water efficiency projects. VA's program is designed to result in accelerated progress in meeting the Federal metering requirements of:

- Install advanced building-level electricity meters by 2012

- Install advanced building-level non-electricity meters by 2016. 


\subsubsection{Approach}

The VA initiated the program in 2006 with the development of an Advanced Meter System Plan. This plan was designed to ask/answer the following questions:

- What utilities and buildings are to be metered?

- What types of meters should be installed?

- How will the metered data be managed?

- How will the metered data be used?

- What are the available funding sources?

- How will the meter installation be prioritized or sequenced?

Once developed and researched, the VA formulated an approach to be both commensurate with internal objectives and in compliance with Federal legislation. As an outcome to this planning process, the VA developed the following criteria:

- Buildings greater than 50,000 gross square feet (gsf) should be metered for electricity, steam, and chilled water (buildings greater than 50,000 gsf comprise 73 percent of VA total square footage).

- Buildings greater than 50,000 gsf using natural gas for heating should be metered; central plants using natural gas should also be metered.

- All buildings with high energy intensity should be metered.

- All incoming utility lines to campuses should be metered.

\subsubsection{Implementation}

Once the planning stage was completed, the activities turned to implementation. This program was designed as a phased approach starting with a pilot phase and followed by two additional phases.

Phase 1: Pilot Project - The first phase was initiated in 2008 and targeted two regions: one large (California) and one small (Ohio). The objectives of this pilot were to design, test, and install a multi-site metering system spanning two states and five different metered resources. The outcome of this effort included:

Central Metering Website - design and installation of a central web-based data collection tool capable of data collection from all existing and proposed metering across the VA.

Meter Installation - installation of over 300 electricity, gas, steam, chilled- and potable- water meters. Meter distribution is as follows:

- Electricity meters - 110 buildings

- Gas meters - 39 buildings

- Steam meters - 43 buildings 
- Chilled water meters - 33 buildings

- Potable water meters - 78 locations.

Data Applications - as part of the pilot design, the uses for data focused on abilities to complete utility bill audits, building benchmarking, and commodity purchasing into the future.

Figure 8.20 represents a schematic of the pilot metering project. Note that the majority of communication was done via wireless networks back to the building management system which serves as a gateway for data export to the central utility monitoring website.
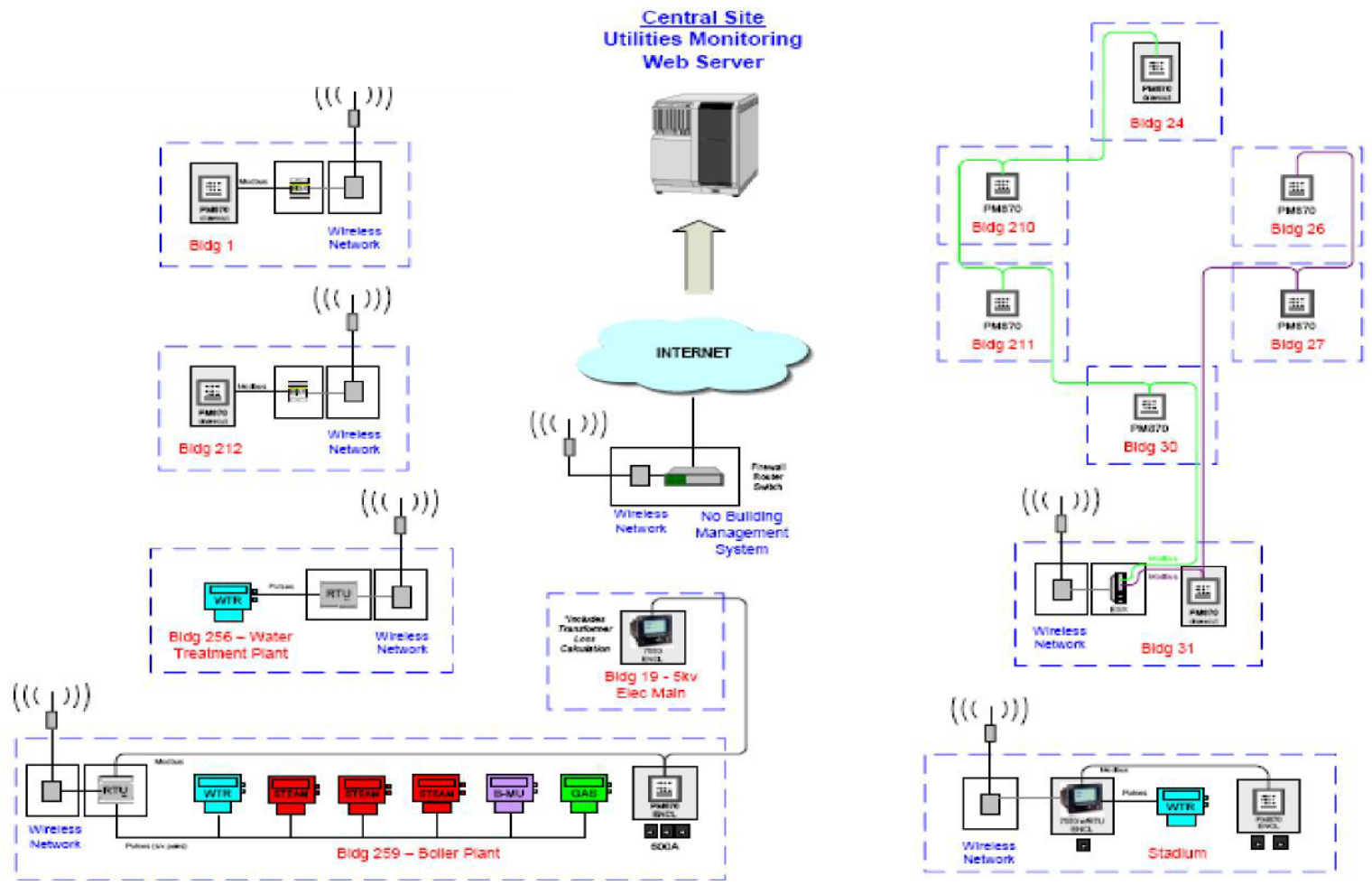

Figure 8.20. Schematic of Veterans Affairs Pilot-Phase Metering Project

Phase 2: National Electric Meter Project - The second phase of the VA metering system focused on electric metering across the entire VA building portfolio. The scope of this project was to install electric meters in the balance of the VA target buildings (greater than 50,000 gsf and/or high electricity usage) and have all data uploaded to the central data collection website.

The Phase 2 installation resulted in an additional 1,742 electric meters installed in VA facilities. The meters were installed across 160 campuses and all metered data are forwarded to the central website.

Figure 8.21 represents a schematic of the Phase 2 project. Wireless networks dominate the onsite communications, including communications from remote buildings and electric feed and substation connections. These are then aggregated at the site gateway and uploaded to the central website. 

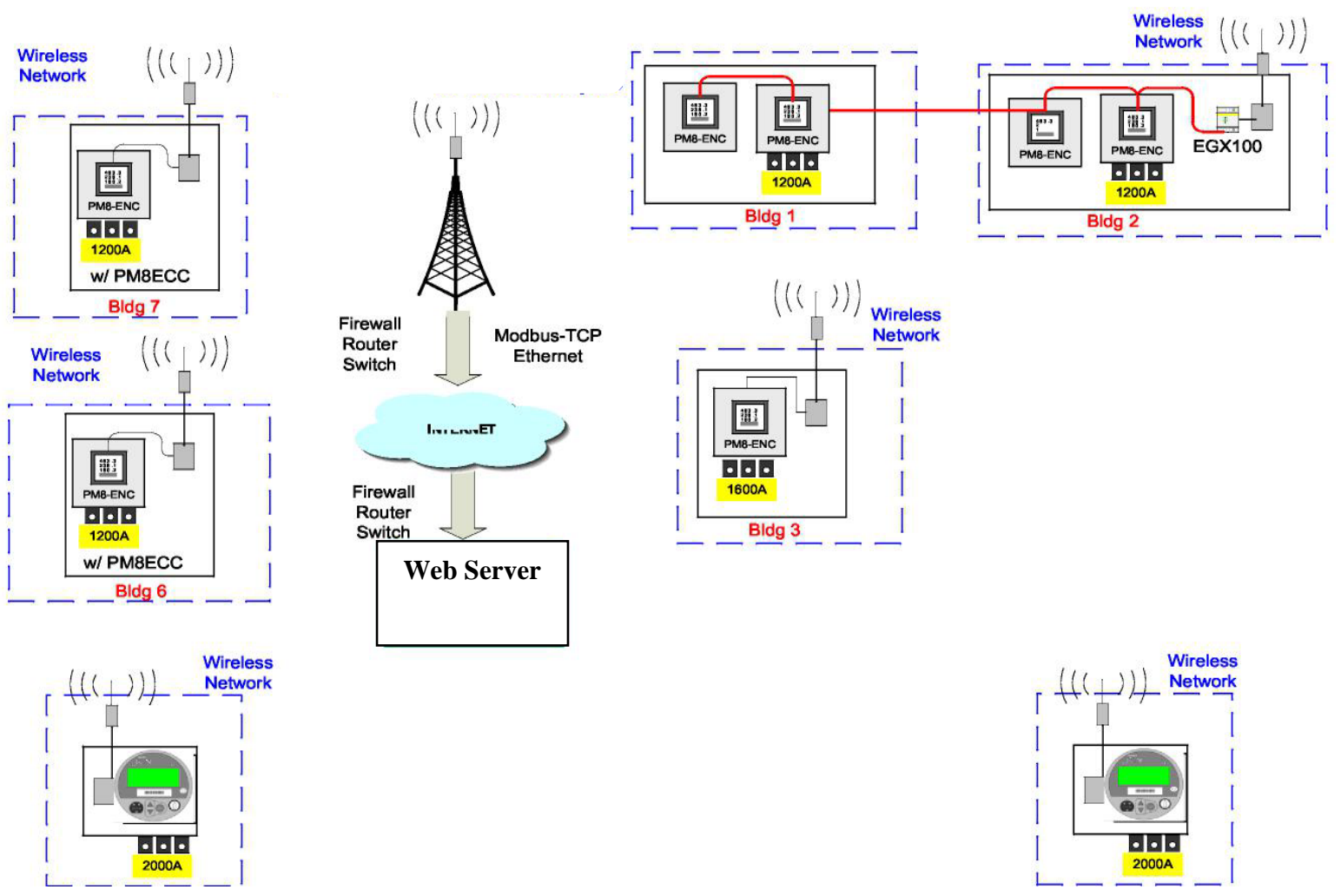

Figure 8.21. Schematic of Veterans Affairs Phase 2: National Electric Metering Project

Phase 3: National Non-Electric Meter Project - The final phase of the project is focused on the installation of non-electric meters across the larger VA building portfolio. This also addresses the same 160 campuses as Phase 2 but features natural gas, steam, chilled water and potable water meters. The installation is expected to be completed in 2011 with an anticipated 3,722 additional meters being installed.

\subsubsection{Findings}

In making use of the central website, the VA has initiated trending activities to identify efficiency opportunities. As more and more data get collected and trended, the VA anticipates significant findings to result.

\section{Success Story 1: Utility Metering Verification}

One early success of the VA's metering and data system involved an invoice validation activity at a Cleveland, Ohio, VA medical center. This center was part of the pilot-phase metering effort where a whole-building natural gas meter was installed. In May 2010, the site energy engineer observed a significantly higher natural gas invoice than was customary for the month of May. The engineer used the enterprise energy management system furnished under the pilot project to determine the monthly gas consumption as measured by the new site natural gas meter. The discrepancy between the natural gas consumption as invoiced from the gas company versus the metered consumption as measured by the VA’s meter was approximately 10,000 Mcf (one Mcf is 1,000 cubic feet of gas). 
To confirm this difference, the data from steam meters (also installed as part of the pilot) were reviewed. These data were used to back-calculate the amount of natural gas that theoretically would have been required to generate the measured quantity of steam produced. There was a strong correlation between the metered natural gas usage and steam produced, indicting an error with the invoiced natural gas consumption.

Based on the ability to assess the situation afforded by the advanced metering, the site engineer submitted a formal inquiry to the utility including all the advanced metering data. This observation was particularly interesting because the natural gas company had installed a new gas meter the previous winter, and there was now a history of several months where the new utility company meter was operating satisfactorily. The utility company submitted a copy of their field readings used to calibrate and certify their new meter to the VA, so the utility company was very hesitant to believe the VA's initial inquiry.

After continued negotiation with the utility, the gas company deployed technicians to the medical center to retest the accuracy of their meter. The spot test conducted by the utility technicians determined the utility company's "new" meter was in fact reading accurately on the day of the spot check. The site engineer then compared the daily natural gas consumption data as provided by the utility for the month, against the daily consumption as measured with the VA's new advanced meters and was able to identify 6 or 7 days where the daily consumption shown in the utility company data was far in excess of the monthly daily average.

After presenting this information to the utility, the utility arranged to have a factory rep from their meter manufacturer visit the site. With some additional investigation, the utility company determined the accuracy of their new "electrical-based" natural gas meter was being compromised by radio-magnetic interference (RMI). The old utility meters were mechanical-based, so they were not affected by the RMI being generated at the medical center. Based on this investigation, the utility company ultimately determined the invoiced consumption was inaccurate for May and June, and issued revised invoices. This resulted in credits of over \$150,000 being applied to the VA, when the combined overbillings of the utility distribution charges and the commodity adjustments were made.

\section{Success Story 2: Capacity Verification and Infrastructure Planning}

The Audie L. Murphy Hospital in San Antonio, Texas, uses district chilled water. The city constructed a municipal chilled water plant in 2005 for the purpose of supporting the San Antonio Medical District. The plant and contract agreement feature peak ton load allocations for each customer. The district chilled water demands have continued to increase each year, pushing the limits of the plant capacity. When plant data indicated that the VA hospital was approaching the allocated limit (4,000 tons), the plant manager initiated discussions to request the VA to plan for a standalone plant on VA property.

The process of producing chilled water involves energy losses from converting electricity for chiller operation to the production of chilled water. In addition, chiller plants lose large amounts of water due to cooling tower evaporation. These utility plant losses are invisible to customers when purchasing chilled water. However, placing a plant behind the VA-owned utility meters would result in a large increase in electric and water usage for the facility. This would make it appear that the facility was going in the wrong direction with respect to conservation goals. 
With the installed Btu chilled water meter system, the VA hospital began monitoring the actual load and total consumption. The summer peak loads were found to be far less than the plant data showed, and much lower than the contract maximum. The VA presented the 15-minute interval data to the district plant manager, who then also installed a meter system with logging capability. Cooperatively, it was documented that the VA actual was closer to a peak of 2,700 tons, and that excess capacity was therefore available to support the VA facility's future expansion projects for the next 10 years.

With the addition of the metering, the hospital also established alarm set points that are triggered when the supply water temperature rises beyond limits or the flow rate is insufficient for air conditioning cooling needs. The alarms have on numerous occasions allowed facility staff to notify the chiller plant of operational discrepancies in advance of any impacts on the space temperatures of the hospital. As a direct result of metering, construction cost savings of over \$5.25 million and manpower requirements for a new VA campus chilled water plant were avoided. Consequently, the Federal goals for energy and water conservation now remain attainable for this hospital.

\subsubsection{Economics}

Metering costs can vary greatly by system configuration, installation and infrastructure needs, and communications options. The VA projects were awarded as complete installations by phase. As such, disaggregated metering component costs are not available. However, the three phases of metering and their associated contract awards are presented below:

- Pilot meter project - approximately $\$ 13$ million

- National electric meter project - approximately \$24 million

- National non-electric meter project - approximately \$92 million.

Long-term cost-effectiveness will be associated with proper use of the metering equipment and applications of data.

\subsubsection{Lessons Learned}

As a result of the VA's extensive metering efforts, a number of lessons learned have been documented. Some of these lessons may be specific to the unique systems the VA operates, but others may apply to most large metering efforts such as this.

- System outage requirements. With the 24/7 nature and critical systems needs in the hospital setting, the VA gravitated toward non-intrusive metering systems as a way to avoid equipment/system shutdowns.

- Increasingly, medical devices are using wireless signals to communicate critical information; these signals cannot be compromised or interfered with by metering systems. Metering systems selected for use in this environment were low-frequency so as not to interfere.

- With flow measurement (chilled water, potable water, and steam) and larger fluctuations in flow rate (i.e., a need for higher turn-down ratios), the VA has had the best success with vortex or magnetic flow meters. 
- Space limitations can dictate meter location and in some cases meter types used.

- When system shutdowns are necessary, proper scheduling is critical and factoring required system “cool-down” is recommended.

\subsection{References}

Curran K. 2007. Advanced Metering Effort: GSA's Plan and Progress to Date. Presented at GovEnergy 2006. Available at http://www.govenergy.com/2007/pdfs/metering/curran_meteringtrack_S9.pdf.

Energy Independence and Security Act of 2007 (EISA 2007). 2007. Public Law 110-140, as amended, Section 434(b).

Energy Policy Act of 2005 (EPAct 2005). 2005. Public Law 109-58, as amended, Section 1252, Smart Metering.

Leifer G. 2006. NIH Case Study. Presented at GovEnergy 2006. Available at: http://www.govenergy.com/2006/pdfs/gregleifer.pdf.

Pacific Northwest National Laboratory (PNNL). 2005. Whole-Building Energy (WBE) Module. Available at: http://eere.pnnl.gov/building-technologies/pdf/application_overview_energy_expert.pdf. 
This page intentionally left blank. 


\section{Appendix A \\ Glossary of Common Terms}


This page intentionally left blank. 


\section{Appendix A}

\section{Glossary of Common Terms}

Advanced meters - Advanced meters are those that have the capability to measure and record interval data (at least hourly for electricity), and communicate the data to a remote location in a format that can be easily integrated into an advanced metering system. EPAct 2005 Section 103 requires at least daily datacollection capability.

Advanced metering system - A system that collects time-differentiated energy or water usage data from advanced meters via a network system on either an on-request or defined schedule basis. The system is capable of providing usage information on at least a daily basis and can support desired features and functionality related to energy-use management, procurement, and operations.

Air conditioning - The process of treating air so as to control its temperature, humidity, and cleanliness while distributing it to cool building space.

Application service provider (ASP) - Third-party entities that manage and distribute software-based services and solutions to customers across a wide-area network from a central data center.

Automated meter reading (AMR) - A form of advanced (or enhanced) metering that uses communications devices to communicate data from the meter to the meter data-management provider. AMR may be used to transmit simple energy-use data from the meter, or to transmit more complex measures of energy recorded in the meter, or to implement advanced functionality, such as outage detection or remote programming.

Average demand - The demand on, or the power output of, an electric system or any of its parts over an interval of time, determined by dividing the number of kilowatt hours by the number of hours in the interval.

Avoided cost - The total economic costs (consisting of the capital and operating costs to provide generation capacity and fuel, transmission, storage, distribution, and customer service) to serve end-use energy requirements using a given set of resources. These costs are referred to as "avoided” when an alternative set of resources is used to serve requirements. A better term for these costs would be "avoidable cost." Avoided cost must be determined to assess the cost-effectiveness of potential supplyside and demand-side resources.

Base load - The minimum average electric load over a given period of time.

Baud rate - The rate of speed at which information is transmitted over communications lines; expressed in bits per second.

Billing demand - The demand for which a customer is billed. Since billing demand is based on the provisions of a rate schedule or contract, it does not necessarily equal the actual measured demand of the billing period. 
Bits - A contraction of binary digits, the smallest unit of information in binary notation. A bit has the value of a zero (0) or a one (1). For example, the binary number 0110 consists of four bits.

British thermal unit (Btu) - A commonly used unit of energy, especially for fuels or heat. A kilowatt hour is equal to approximately $3412 \mathrm{Btu}$. Quantity of heat required to raise one pound of water by one degree Fahrenheit or the equivalent amount of energy generated by burning a kitchen match.

Building envelope - The exterior surfaces of a building, such as the roof, walls, windows, doors, etc., that are exposed to climatic conditions.

Capacity - The maximum quantity of electrical output for which a supply system or component is rated.

ccf - Hundred cubic feet.

$\mathbf{c f m}$ - Cubic feet per minute.

Coincident demand - Two or more demands that occur during the same time interval. Often used to express the demand level of subgroups of customers that occurs at the time of the electric system's overall maximum peak demand.

Constant dollars - Monetary value based on the purchasing power within the base year-inflationary impacts are not reflected in the value of the constant dollars.

Control - Any manual or automatic device designed to regulate the operation of a system or system component.

Cooling loads - The energy required to achieve the desired (space cooling) temperature level.

Cost avoidance - In regard to energy efficiency - the implementation of energy saving measures will result in a dollar savings which will offset any fuel price increase.

Cost-effective - The present value (PV) of the benefits of the potential resource under consideration over the planning period are greater than the PV of its costs. Cost-effectiveness is always measured relative to an alternative. Cost-effectiveness can be measured from a variety of perspectives, which vary in terms of the specific costs and benefits included in the calculation.

Damper - A valve or movable plate that is attached to a duct in order to regulate the flow of air or other gases.

Degree-days (cooling) - The difference between the average temperature of any given day and a base temperature when the median temperature of the given day is higher than the base temperature.

Degree-days (heating) - The difference between the average temperature on any given day and a base temperature when the median temperature of the given day is less than the base temperature. Often the base temperature selected is $65^{\circ} \mathrm{F}$.

Dehumidification - The process of removing moisture. 
Demand - The rate at which electricity is delivered by a system or part of a system, or to a load point or set of loads. It is measured in kilowatts, kilovolt amperes, or other suitable unit at a given instant or averaged over a designated period of time.

Dew point temperature - The temperature level in which the moisture in the air begins to condense.

Diversity - The diversity among customers' demands, which creates variations among the loads in distribution transformers, feeders, and substations at a given time. A load diversity is the difference between the sum of the maximum of two or more individual loads and the coincident or combined maximum load. It is usually measured in kilowatts.

Domestic hot water system (DHW) - A system designed to provide hot water for domestic needs - the energy required for a DHW system will vary according to building size, design, and human needs.

Dry bulb temperature - The temperature level as measured on a standard thermometer.

Duct work - A series of piping in which air is transferred from its source to the space that is to be conditioned. Ducts, which should be insulated to improve their efficiency, are generally made from fiberglass or galvanized metal.

Economizer cycle - Use of outside air without further mechanical cooling for space cooling when conditions are appropriate. Typically, this is accomplished by locking out the cooling coil.

Electric utility - A corporation, person, agency, authority, or other legal entity or instrumentality that owns and/or operates facilities within the United States, its territories, or Puerto Rico for the generation, transmission, distribution, or sale of electric energy primarily for use by the public and files forms listed in the Code of Federal Regulations, Title 18, Part 141. Facilities that qualify as co-generators or independent power producers under the Public Utility Regulatory Policies Act (PURPA) are not considered electric utilities. Electrical energy is usually measured in kilowatt-hours, whereas heat energy is usually measured in British thermal units.

Emission factor - The ratio of emissions to energy produced or fuel consumed, denominated in units of tons of emissions per unit of energy.

End-use - Useful work, such as light, heat, and cooling, which is produced by electricity or other forms of energy.

Energy - The capacity for doing work as measured by the capability of doing work (potential energy) or the conversion of this capability to motion (kinetic energy). Energy has several forms, some of which are easily convertible and can be changed to another form useful for work. Most of the world's convertible energy comes from fossil fuels that are burned to produce heat that is then used as a transfer medium to mechanical or other means in order to accomplish tasks.

Energy audit - Analysis of a facility's electricity and other energy usage, often including recommendations to alter the customer's electric demand or reduce energy usage. An audit usually is based on a visit by an energy analyst or engineer to the home, building, or manufacturing or agricultural facility. 
Energy charge - The charge for electric service based upon the amount of electric energy (kWh) consumed and billed under an applicable rate schedule.

Energy cost liability - Estimated future energy expenditures without energy saving improvements.

Energy management system - A full or partially computerized system designed to monitor and control energy use in order to achieve optimal efficiency.

Energy use index (EUI) - Annual Btu/square foot energy use. The standard index used in most analyses to measure all fuel and energy used in a given building or group of buildings.

Ethernet - A specification for local communication networks that employs cable as a passive communication medium to interconnect different kinds of computers, information processing products, and office equipment at a local site.

Extensible markup language (XML) - Specification that allows designers to create their own customized tags, enabling the definition, transmission, validation, and interpretation of data between applications and between organizations.

Firewall - A system designed to prevent unauthorized access to or from a private network. Firewalls can be implemented in both hardware and software, or a combination of both.

Gateway - In Local Area Networks (LANs), a computer system and its associated software that permit two networks using different protocols to communicate with each other. A gateway translates all protocol levels from physical layer up through applications layer and can be used to interconnect networks that differ in every detail.

Hourly metering - A type of interval metering where the measurement or recording of customer use is collected in 6-minute intervals. The competitive metering model is based upon the implementation of hourly metering of customers or the application of load profiles, which average customer use over hourly periods.

Hypertext transfer protocol - The underlying protocol used by the World Wide Web. Hypertext transfer protocol defines how messages are formatted and transmitted and what action Web servers and browsers should take in response to various commands.

Incremental cost - The difference in costs between two alternatives, for example, between that of an efficient technology or measure and the standard technology.

Instantaneous peak demand - The demand at the instant of greatest load, usually determined from the readings of indicating meters or graphic meters.

Integrated demand - The summation of continuously varying instantaneous demands during a specified demand interval.

Interconnection - The physical connection of the customer's generation source to a utility's distribution network. Implicit in this are the requirements and protocols for code compliance and safety standards. 
Interface - A device that allows communication between systems or ports of systems.

Interval metering - The measurement of customer energy use by fixed time periods or intervals. Typically, the interval time period is 15 minutes, but can vary according to the customer or transmission and distribution system needs. Today, interval metering is provided to commercial and industrial customers and some residential customers. In the future, in an unbundled environment, the residential market may require more frequent interval measurements.

IP address - Internet protocol address. See also Ethernet.

Islanding - The condition in which onsite power is still being generated while the grid is down. This situation creates a variety of safety and equipment concerns and is usually a stipulated code issue. Many modern onsite power systems have (as are required) anti-islanding mechanisms that cease to energize upon detection of line voltage/frequency anomalies.

Kilowatt (kW) - One thousand watts.

Kilowatt-hour (kWh) - A standard unit of energy equivalent to a demand rate of 1,000 watts over 1 hour.

Levelized cost - The uniform annual cost that results in the same net present value over the planning horizon as the stream of actual annual average costs. An example of a levelized cost is a monthly mortgage payment.

Life-cycle costing (LCC) - The analytical process for estimating the total cost of a product or system over the life of the product or system, including the operational and maintenance costs.

Line losses - Kilowatt-hours and kilowatts lost in the transmission and distribution lines under specified conditions.

Load - The amount of electric power consumed at any specified point or points on a system. Load originates primarily in the power consuming equipment of the customers.

Load aggregation - Aggregation of energy consumption from facilities that are geographically separate from each other for purposes of acquiring and billing utility services.

Load duration curve - A graph showing a utility's hourly demand, sorted by size, as well as by the amount of time a given level of demand is exceeded during the year.

Load factor - The ratio of the average load in kilowatts supplied during a given period to the peak or maximum load in kilowatts occurring during that period. Load factor may be calculated for a customer, customer class, or the entire electric system.

Load leveling - A process in which the energy demand can be temporarily reduced during certain periods. Typical examples include the intermittent operation of certain electrical equipment and shutting off equipment when rooms or buildings are not in use. 
Load management - The controlling, by rescheduling or direct curtailment, of the power demands of customers or groups of customers in order to reduce the total load that a utility must meet at times of peak demand. Load management strategies are designed to either reduce or shift demand from on-peak to offpeak, while conservation strategies reduce usage over larger multi-hour periods. Load management may take the form of normal or emergency procedures. Utilities often encourage load management by offering customers a choice of service options with varying price incentives.

Local area network (LAN) - Computer network that spans a relatively small area.

Megawatt (MW) - One million watts.

Mcf - Thousand cubic feet.

Modbus ${ }^{\circledR}$ protocol - A messaging structure developed by Modicon in 1979, used to establish masterslave/client-server communication between intelligent devices. It is a de facto standard, truly open, and the most widely used network protocol currently available.

Modem - Modulator-demodulator. A device or program that enables a computer to transmit data over telephone lines.

Multipoint communications - A method of communication in which a single device can communicate to multiple devices.

MV-90 - The utility industry de facto standard for data collection and storage systems. This system was developed so that meters from different vendors could be read and the data stored in a consistent manner.

NEMA standards - Property characteristics adopted as standard by the National Electrical Manufacturers Association.

Net metering - A utility procedure for crediting customers for electricity generated onsite and in excess of their own needs. In its simplest form, this allows for the flow of electricity both to and from a customer's location through a single bi-directional meter.

Net present value (NPV) - The value of future energy savings-less all project construction and operating costs, discounted to present value.

Network - A group of computing devices that are connected to each other by communications lines to share information and resources.

Nominal levelized cost - The uniform cost of electricity, in mixed current dollars, for which the present value of the cost of electricity produced over the life of the plant is equal to the present value of the costs of the plant.

Non-volatile memory - Memory that retains its contents when power is lost.

Off-peak energy - Electricity supplied during periods of relatively low system demand. 
Peak demand - The maximum rate of electricity consumption, expressed in gigawatts or kilowatts during a specified period of time. May be expressed for groups of electricity users or the whole system, and by season (summer or winter) or annually. See demand. Also called peak load.

Peak load - The maximum anticipated demand for any given system.

Peaking unit, or peaker - A generating station that is normally operated to provide power during maximum load periods.

Planning period - The time period over which the utility Integrated Resource Planning (IRP) analysis is performed.

Potential resources - Resources, either supply-side or demand-side, which are either currently commercially available, feasible, or are expected to be commercially available within the planning period.

Power Line Carrier (PLC) - Communication system that transmits data between devices over power lines.

Present value - The value of a cost or stream of yearly costs that have been discounted to reflect the fact that future benefits or expenditures are worth less than current benefits or expenditures. Also called present worth.

Programmable Logic Controller (PLC) - A solid-state control system that has a user programmable memory for storage instruction to implement specific functions such as input/output (I/O) control logic, timing, counting, arithmetic, and data manipulation.

Protocol - A standardized procedure for establishing a communications link between devices and that is based on such elements as word structure or word length.

Radio frequency (RF) - Refers to alternating current (AC) having characteristics such that, if the current is input to an antenna, an electromagnetic (EM) field is generated suitable for wireless broadcasting and/or communications.

Real levelized cost - The uniform cost of electricity, in constant dollars, for which the present value of the electricity produced equals the present value of the costs of the plant. See also the levelization formulae following this glossary.

Real-time metering - Metering that records consumer use in the same time frame as pricing changes in the market, typically hourly or more frequently.

Real-time pricing (RTP) - The instantaneous pricing of electricity based on the cost of electricity available for use at the time the electricity is demanded by the customer.

Relative humidity (RH) - The percentage of moisture contained in the air compared to saturation.

Retrofit - Energy saving improvements to a building structure or any of its energized systems involving modification of that structure or system. 
Return on investment (ROI) - The discount rate which, when used to discount all present and future project costs and savings, brings the net present value to zero.

RS-485 serial communications bus - An Electronics Industry Association (ETA) standard for multipoint communications. RS-485 is similar to RS-422 but can support more nodes per line because it uses lower-impedance drivers and receivers.

Server - A computer or device on a network that manages network resources. For example, a file server is a computer and storage device dedicated to storing files.

Shadow metering - The process to quantify and verify the system generation and assure proper "net metering” credit.

Transmission control protocol/internet protocol (TCP/IP) - The suite of communications protocols used to connect hosts on the Internet. TCP/IP uses several protocols, the two main ones being TCP and IP. TCP/IP is built into the UNIX operating system and is used by the Internet, making it the de facto standard for transmitting data over networks.

therm - Equals 100,000 Btus.

Time of use - The pricing of electricity based on the estimated cost of electricity during a particular time block.

Tons of refrigeration - A standard for identifying cooling capacity. One ton of refrigeration is equal to 12,000 Btu/hour of cooling.

Utility discount rate - A rate that reflects the utility's weighted cost of capital. Pre-tax or, more commonly, after tax.

Valley filling - The building of off-peak loads. An example of valley filling technology is thermal storage (water heating and/or space heating or cooling) that increases nighttime loads and reduces peak period loads. Valley filling may be desired in periods when the long-run incremental cost of supply is less than the average price of electricity. (Adding off-peak load under those circumstances decreases the average price.)

Variable operating and maintenance $(\mathbf{O} \& \mathbf{M})$ costs - The additional cost per kWh of electricity produced that goes toward operation and maintenance of the plant. These costs vary with the output of the plant and are expressed in cents per kWh of electricity produced.

Watt - The electrical unit of power. The rate of energy transfer equivalent to 1 ampere flowing under a pressure of 1 volt at unity power factor.

Watt-hour - The total amount of energy used in one hour by a device that requires one watt of power for continuous operation. Electric energy is commonly sold by the kilowatt-hour (1,000 Watt-hour). 


\section{Appendix B}

\section{Legislative Requirements}


This page intentionally left blank. 


\section{Appendix B}

\section{Legislative Requirements}

Energy Policy Act of 2005

\section{SEC. 103. ENERGY USE MEASUREMENT AND ACCOUNTABILITY.}

Section 543 of the National Energy Conservation Policy Act (42 USC 8253) is further amended by adding at the end of the following.

\section{“(e) METERING OF ENERGY USE.-}

“(1) DEADLINE.-By October 1, 2012, in accordance with guidelines established by the Secretary under paragraph (2), all Federal buildings shall, for the purposes of efficient use of energy and reduction in the cost of electricity used in such buildings, be metered. Each agency shall use, to the maximum extent practicable, advanced meters or advanced metering devices that provide data at least daily and that measure at least hourly consumption of electricity in the Federal buildings of the agency. Such data shall be incorporated into existing Federal energy tracking systems and made available to Federal facility managers.

\section{“(2) GUIDELINES.-}

“(A) IN GENERAL.- -Not later than 180 days after the date of enactment of this subsection, the Secretary, in consultation with the Department of Defense, the General Services Administration, representatives from the metering industry, utility industry, energy services industry, energy efficiency industry, energy efficiency advocacy organizations, national laboratories, universities, and Federal facility managers, shall establish guidelines for agencies to carry out paragraph (1).

“(B) REQUIREMENTS FOR GUIDELINES.-The guidelines shall-

“(i) take into consideration-

"(I) the cost of metering and the reduced cost of operation and maintenance expected to result from metering;

"(II) the extent to which metering is expected to result in increased potential for energy management, increased potential for energy savings and energy efficiency improvement, and cost and energy savings due to utility contract aggregation; and "(III) the measurement and verification protocols of the Department of Energy; 
"(ii) include recommendations concerning the amount of funds and the number of trained personnel necessary to gather and use the metering information to track and reduce energy use;

“(iii) establish priorities for types and locations of buildings to be metered based on cost-effectiveness and a schedule of one or more dates, not later than 1 year after the date of issuance of the guidelines, on which the requirements specified in paragraph (1) shall take effect; and

“(iv) establish exclusions from the requirements specified in paragraph (1) based on the de minimis quantity of energy use of a Federal building, industrial process, or structure.

“(3) PLAN.- - Not later than 6 months after the date guidelines are established under paragraph (2), in a report submitted by the agency under section 548(a), each agency shall submit to the Secretary a plan describing how the agency will implement the requirements of paragraph (1), including (A) how the agency will designate personnel primarily responsible for achieving the requirements and (B) demonstration by the agency, complete with documentation, of any finding that advanced meters or advanced metering devices, as defined in paragraph (1), are not practicable.

Energy Independence and Security Act of 2007

\section{SEC. 434. MANAGEMENT OF FEDERAL BUILDING EFFICIENCY.}

METERING.--Section 543(e)(1) of the National Energy Conservation Policy Act (42 USC 8253(e)(1)) is amended by inserting after the second sentence the following: "Not later than October 1, 2016, each agency shall provide for equivalent metering of natural gas and steam, in accordance with guidelines established by the Secretary under paragraph (2).'’

From NECPA, the information regarding paragraph 2 is provided below:

(2) Guidelines

(A) In general

Not later than 180 days after August 8, 2005, the Secretary, in consultation with the Department of Defense, the General Services Administration, representatives from the metering industry, utility industry, energy services industry, energy efficiency industry, energy efficiency advocacy organizations, national laboratories, universities, and Federal facility managers, shall establish guidelines for agencies to carry out paragraph (1).

(B) Requirements for guidelines

The guidelines shall-

(i) take into consideration--

(a) the cost of metering and the reduced cost of operation and maintenance expected to result from metering; 
(b) the extent to which metering is expected to result in increased potential for energy management, increased potential for energy savings and energy efficiency improvement, and cost and energy savings due to utility contract aggregation; and

(c) the measurement and verification protocols of the Department of Energy;

(ii) include recommendations concerning the amount of funds and the number of trained personnel necessary to gather and use the metering information to track and reduce energy use;

(iii) establish priorities for types and locations of buildings to be metered based on costeffectiveness and a schedule of one or more dates, not later than 1 year after the date of issuance of the guidelines, on which the requirements specified in paragraph (1) shall take effect; and

(iv) establish exclusions from the requirements specified in paragraph (1) based on the de minimis quantity of energy use of a Federal building, industrial process, or structure. 
This page intentionally left blank. 


\section{Appendix C}

\section{Metering Codes and Standards}


This page intentionally left blank. 


\section{Appendix C}

\section{Metering Codes and Standards}

Codes and standards applicable to metering equipment and installation are generally governed and promulgated by national organizations and enforced by local entities such as building code officials.

Because different parts of the country have traditionally referenced different code organizations for specific codes, the authors of this guide recommend contacting local building code officers (electric, natural gas, and plumbing inspectors) prior to initiating any meter selection or installation project.

Below are presented some of the more common standards as they apply to the major metering categories.

\section{Electric Meters and Installation Codes and Standards}

All electric meters installed must comply with the National Electric Code (NEC) as found in the National Fire Protection Association (NFPA) 70. Additional standards from the American National Standards Institute (ANSI) are provided below.

American National Standards Institute (ANSI) C12 metering standards:

- ANSI C12.1 - American National Standard Code for Electricity Metering

- ANSI C12.4 - American National Standard for Mechanical Demand Registers

- ANSI C12.5 - American National Standard for Thermal Demand Meters

- ANSI C12.6 - American National Standard for Marking and Arrangement of Terminals

\section{Self-Contained A-Base Watt-Hour Meters}

- ANSI C12.9 - American National Standard for Test Switches for Transformer-Rated Meters

- ANSI C12.10 - American National Standard for Electromechanical Watt-Hour Meters

- ANSI C12.11 - American National Standard for Instrument Transformers for Revenue Metering, 10 kV BIL Through 350 kV BIL

- ANSI C12.13 - American National Standard for Electronic Time-of-Use Registers for Electricity Meters

- ANSI C12.14 - American National Standard for Magnetic Tape Pulse Recorders for Electricity Meters

- ANSI C12.15 - American National Standard for Solid-State Demand Registers 


\section{Electromechanical Watt-Hour Meters}

- ANSI C12.16 - American National Standard for Solid-State Electricity Meters

- ANSI C12.17 - American National Standard for Cartridge-Type Solid-State Pulse Recorders

The Institute of Electrical and Electronics Engineers (IEEE) has standards related to electromagnetic immunity including:

- IEEE C.37-90.1-1989 - IEEE Standard Surge Withstand Capability (SWC) Tests for Protective Relays and Relay Systems (ANSI). All inputs tested, except for the network communications port.

ANSI is a member of the IEC (International Electrotechnical Commission), which has adopted the following standards related to metering:

- IEC1000-4-2 (EN61000-4-2/IEC801-2) - Electrostatic Discharge (B)

- IEC1000-4-3 (EN61000-4-3/IEC801-3) - Radiated EM Field Immunity (A)

- IEC1000-4-4 (EN61000-4-4/IEC801-4) - Electric Fast Transient (B)

- IEC1000-4-5 (EN61000-4-5/IEC801-5) - Surge Immunity (B). Certified by American Electric Power (AEP)

- IEC1000-4-6 (EN61000-4-6/IEC801-6) - Conducted Immunity

- IEC 60687 0.2S, section 4.6.1, 4.6.2, 4.6.3.

The Federal Communications Commission (FCC) also regulates electromagnetic emission:

- FCC Part 15 Subpart B, Class A: Class A Digital Device, Radiated Emissions. d. IEC Compliance.

Other organizations might impose local requirements. For example, the California Independent System Operator (ISO) has standards and protocols for installing, reading, and maintaining meters on the system including:

- ISO MTR1-96 - Engineering Specifications for Poly-Phase Solid-State Electricity Meters for use on the ISO Grid.

Many of the standards addressed above are very specific and not always applicable. Their presentation highlights the depth to which metering and communication standards apply. As mentioned, the best resource may well be a local building code official for these and other code/standard issues and questions.

\section{Natural Gas Meter and Installation Codes and Standards}

All natural gas meters installed must comply with the National Fuel Gas Code as found in the National Fire Protection Association (NFPA) 54. Additional codes and standards may apply as issued by state and local code authorities. The best resource for complete and up-to-date code information may well be a local building code official. 


\section{Water Meter and Installation Codes and Standards}

Water meters and installation are usually governed by the Uniform Plumbing Code but will often be superseded/amended by local ordinances specific to the region. The best resource for complete and up-todate code information will be a local building code official. 
This page intentionally left blank. 


\section{Appendix D}

\section{Suggestions for Additions or Revisions}


This page intentionally left blank. 


\section{Appendix D}

\section{Suggestions for Additions or Revisions}

This guide is a living document, open to periodic updates and improvement. Readers are encouraged to submit suggestions for additions, deletions, corrections, or where to go for other resources.

In addition, we are interested in what has worked at your Federal site. We want to find other case studies and documentation of your successes.

Please send or fax your information to:

David Hunt

Pacific Northwest National Laboratory (PNNL)

Battelle Washington Operations

Suite 900901 D Street SW

Washington, DC 20024-2169

Fax: (202) 646-7825

Additional material to include (please be specific):

Additional references/resources:

Case study material (feel free to attach additional sheets): 
This page intentionally left blank. 


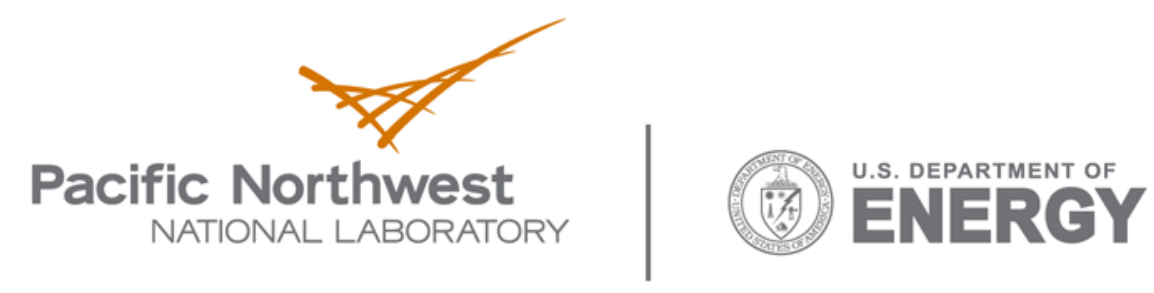

902 Battelle Boulevard

P.O. Box 999

Richland, WA 99352

1-888-375-PNNL (7665)

www.pnl.gov 